MATHEMATICAL CENTRE TRACTS 103

\title{
CLASSIFYING INFINITELY DIVISIBLE DISTRIBUTIONS BY FUNCTIONAL EQUATIONS
}

K. VAN HARN 
AMS (MOS) subject classification scheme (1970I: 60E05, 60F05, 60G50, 60K05; $26 \mathrm{~A} 48 ; 39 \mathrm{~A} 15 ; 44 \mathrm{~A} 10,44 \mathrm{~A} 35$

ISBN $906196 \quad 1726$ 
CONTENTS

CONTENTS

ACKNOWLEDGEMENTS

CHAPTER 1. INTRODUCTION AND PRELIMINARIES

1.1. Introduction and summary

1.2. Notations and conventions

1.3. Absolute and complete monotonicity

1.4. Definition and basic properties of infinitely divisible distributions on $\mathbb{R}$

1.5. Infinitely divisible lattice distributions

1.6. Infinitely divisible distributions on $[0, \infty)$

1.7. Properties of and relations between canonical representations

CHAPTER 2. CLASSIFICATION OF THE INFINITELY DIVISIBLE LATTICE DISTRIBUTIONS

2.1. Interpolating between $C_{0}$ and $C_{1}$

2.2. The choice of $\mathrm{C}_{\mathrm{n}}^{*}(\alpha)$

2.3. The classes $C_{\alpha}$; basic properties 45

2.4. Further properties and examples 51

2.5. Other classifications 63

CHAPTER 3. DECOMPOSITIONS OF LATTICE DISTRIBUTIONS

3.1. a-decomposable and a-factorizable lattice 69 distributions

3.2. Totally decomposable and totally factorizable lattice distributions

3.3. Discrete self-decomposability and stability 85

3.4. a-decomposable(1) lattice distributions 95 
CHAPTER 4. THE CLASSES $C_{\alpha}$ IN RELATION TO RENEWAL THEORY 4.1. The class $C_{0}$ and discrete-time renewal theory 99

4.2. Generalized renewal sequences; classes $R \quad(0<\alpha<1) \quad 110$

4.3. An extension of $C_{1}$; the class $R_{1}$

CHAPTER 5. CLASSIFICATION OF THE INFINITELY DIVISIBLE DISTRIBUTIONS ON $[0, \infty)$

5.1. The classes $F$; preliminaries 134

5.2. The monotonicity of $F$, absolute continuity 140

5.3. Further properties of the $F_{\lambda}^{\prime}$ 's, examples 146

5.4. The class $F_{\infty} 154$

5.5. The class $F_{\infty}$ in relation to standard p-functions 177

5.6. Other classifications 185

5.7. Further generalizations 187

REFERENCES 


\section{ACKNOWLEDGEMENTS}

This monograph is a result of my research during the years 1974 until 1978, carried out at the Department of Mathematics of the Eindhoven University of Technology. Its subject-matter was suggested by my thesis advisor Dr. F*W. steutel, to whom I am very grateful for continual encouragement and many helpful discussions. I am also indebted to my co-promotor Prof.dr. R. Doornbos, Prof.dr.ir. M.L.J. Hautus and Dr. W. Vervaat, who, despite pressure of work, found time to critically read the manuscript, and made several useful suggestions.

Further I would like to thank Mr. I.G.F.C. van Bree, who did the programming needed in chapter 2, and Mrs. Th.J.M. Wolfs for her quick and excellent typing of the manuscript.

Finally I am indebted to the Mathematical Centre for the opportunity to publish this monograph in their series: Mathematical Centre Tracts, and to all those at the Mathematical Centre who have contributed to its technical realization. 



\section{CHAPTER 1}

\section{INTRODUCTION AND PRELIMINARIES}

\subsection{Introduction and summary}

The theory of infinitely divisible probability distributions plays an important role in theoretical problems, such as in the study of limit theorems, more so than in practical situations, though applications do occur, especial$1_{y}$ in statistical modelling (cf. Katti (1977), Thorin (1977) and Ahmad \& Abouammoh (1977)). The first stage of its development ended around 1950; the basic properties, such as canonical representations, derived especially by $P$. Lêvy and I.A. Khintchine, and the important applications in the theory of limit distributions of sums of independent random variables, have been formulated, for instance, in the books by Lévy (1937) and by Gnedenko \& Kolmogorov (1949). In the next two decades research on this field has been carried out along many lines; especially, much attention has been paid to factorization problems and stable distributions, as is apparent from the survey paper by Fisz (1962) and from the books by Linnik (1960) and Lukacs (1970). For more recent information we refer to Petrov (1972). During the last ten years more research has been done on the often difficult problem how to decide whether a given probability distribution is infinitely divisible or not. On the one hand new methods of constructing infinitely divisible distributions have been introduced; for instance, the methods of compounding and mixing are very useful, as has been shown by steutel (1970), Kelker (1972) and others. On the other hand many necessary and (or) sufficient conditions for infinite divisibility have been obtained in terms of the probabilities themselves, rather than in terms of the corresponding characteristic functions, the most obvious tool in this field; this is evident from the survey paper by steutel (1973).

In this monograph this tendency is continued in the following sense: most of the classes of infinitely divisible probability distributions that we introduce, are characterized by means of functional equations for the probabilities themselves; furthermore, we study properties of distribution functions and densities in these classes, like asymptotic behaviour, absolute continuity, complete monotonicity, etc.

our starting point is the "gap" between the class $C_{0}$ of compound geometric distributions on $\mathbb{N}_{0}$ and the class $C_{1}$ of compound Poisson distributions on $\mathbb{N}_{0}$ i.e. (cf. Feller (1968), ch. XII) the class of all infinitely divisible 
distributions on $\mathbb{N}_{0}$ with factors in $\mathbb{N}_{0}$. It is known that $C_{0} \neq C_{1}$ (cF. Lukacs (1970), ch. 5). Furthermore, the classes $C_{0}$ and $C_{1}$ can be characterized by means of recurrence relations as follows (cf. Steutel (1970) and Katti (1967)): a probability distribution $\left\{p_{n}\right\}_{0}^{\infty}$ on $\mathbb{N}_{0}$ with $p_{0}>0$ is in $C_{0}$ iff there exist nonnegative quantities $r_{n}(0)\left(n \in \mathbb{N}_{0}\right)$ such that

(1.1.1) $\quad p_{n+1}=\sum_{k=0}^{n} p_{k} x_{n-k}(0) \quad\left(n \in \mathbb{N}_{0}\right)$

similarly, $\left\{p_{n}\right\}$ is in $C_{1}$ iff there exist nonnegative quantities $r_{n}(1)\left(n \in \mathbb{N}_{0}\right)$ such that

$(1.1 .2) \quad(n+1) p_{n+1}=\sum_{k=0}^{n} p_{k} r_{n-k}(1) \quad\left(n \in \mathbb{N}_{0}\right)$.

Now, in order to fill the gap between $C_{0}$ and $C_{1}$ we intexpolate between $(1.1 .1)$ and $(1.1 .2)$ by means of a set of recurrence relations of the following form:

(1.1.3) $\quad c_{n}(\alpha) p_{n+1}=\sum_{k=0}^{n} p_{k}{ }^{r} n-k(\alpha) \quad\left(n \in \mathbb{N}_{0}\right)$

where $c_{n}(0)=1, c_{n}(1)=n+1\left(n \in \mathbb{N}_{0}\right)$ and $c_{n}(\alpha)$ is nondecreasing in both $n$ and $\alpha \in[0,1]$. Introducing for $0<\alpha<1$ the class $C_{\alpha}$ as the set of distributions $\left\{p_{n}\right\}_{0}^{\infty}$ with $p_{0}>0$ and satisfying (1.1.3) with nonnegative $x_{n}(\alpha)$ " $s_{\text {, }}$ we wish to choose $c_{n}(\alpha)$ in such a way that the $C_{\alpha}$ "s yield a classification of $C_{1}, i . e$. such that $C_{\alpha}$ depends monotonically on $\alpha \in[0,1]$. The most obvious choices for $c_{n}(\alpha)$ do not have this property, but in chapter 2 we show that the choice

$(1.1 .4) \quad c_{n}(\alpha)=\left(1-\alpha^{n+1}\right) /(1-\alpha) \quad\left(n \in \mathbb{N}_{0} ; 0 \leq \alpha \leq 1\right)$

produces classes $C_{\alpha}$ that give a classification of $C_{1}$. Rather surprisingly, perhaps, we did not find any other. It would seem that these C "s are "Clom ser" to $C_{0}$ than to $C_{1}$, but as $U_{\alpha<1} C_{\alpha}$ is dense in $C_{1}$ in the sense of weak convergence, the situation is not too bad. Also in this chapter we briefly consider some other classifications.

Furthermore, the classes $C_{\alpha}$ give rise to a number of other interesting observations. The equations defining the probability generating functions of distributions in $C_{\alpha}$ suggest several other classes of decomposable distributions; these are studied in chapter 3. One of these gives rise to discrete analogues of the well known concepts of self-decomposability and stability 
(cf. Lukacs (1970), ch. 5 and Feller (1971), ch. XVII), concepts which were restricted to absolutely continuous aistributions.

In chapter 4 we investigate the recurrence relations (1.1.3) with $c_{n}(\alpha)$ given by $(1.1 .4)$ for sequences $\left\{p_{n}\right\}$ that are not necessarily probability distributions. Several properties can be proved that are analogous to properties of the sequences studied by Kaluza (1928) and De Bruijn \& Erdös (1951). Also, we show a fruitful relation with renewal theory; it turns out that the case $\alpha=0$ is strongly related to the renewal sequences (cf. Kingman (1972)), while fox $0<\alpha<1$ the bounded solutions of (1.1.3) with nonnegam tive $r_{n}(\alpha)^{\prime}$ s can be considered as delayed renewal sequences. Although, especially from these relations, several properties can be obtained, it turns out that the case $0<\alpha<1$ is often difficult to handle; in many respects this case seems to inhexit the difficulties of both the cases $\alpha=0$ and $\alpha=1$. In chapter 5 the classification of $C_{1}$, obtained by means of the classes $C_{\alpha}$ is extended to all infinitely divisible distributions on $[0, \infty)$, by replacing the system ofrecurrence relations (1.1.3) for $p_{n}$ by the analogous functional equation for the distribution function. As, contrary to the discrete case, we also have to consider distributions on $[0, \infty)$ without a jump at zero, the proofs are more delicate and the analogy with the discxete case is not perfect. At this point it is interesting to note that the resulting classes determine a limiting class $F_{\infty}$ that can be considered as the analogue of $C_{0}$ for distributions on $[0, \infty)$, just as the class of all infinitely divisible distributions on $[0, \infty)$ is the analogue of $C_{1}$. A good deal of chapter 5 is devoted to investigating the structure and properties of this class $F_{\infty}$. It turns out that the absolutely continuous elements of $F_{\infty}$ contain the standard p-functions of Kingman (1972) as a subclass. Finally, in the last section of chapter 5 we briefly discuss the classification of the infinitely divisible distributions on $\mathbb{R}$, and on $[0, \infty)^{2}$, by means of functional equations.

The remainder of the present chapter contains definitions and preliminary results. After some notations and conventions in section 2, in section 3 we introduce the concepts of absolute and complete monotonicity, which we shall use frequently. The concept of infinite divisibility and its basic properties are introduced in section 4 , where also some attention is paid to compound distributions. In sections 5 and 6 we study the infinitely divisible distributions on $\mathbb{I N}_{0}$ and on $[0, \infty)$, respectively, in more detail. Finally, in section 7 we give a survey of canonical representations for infinitely divisible distributions and the relations between them. 


\subsection{Notations and conventions}

First we give a list of general symbols and notations, which we shall use throughout this monograph.

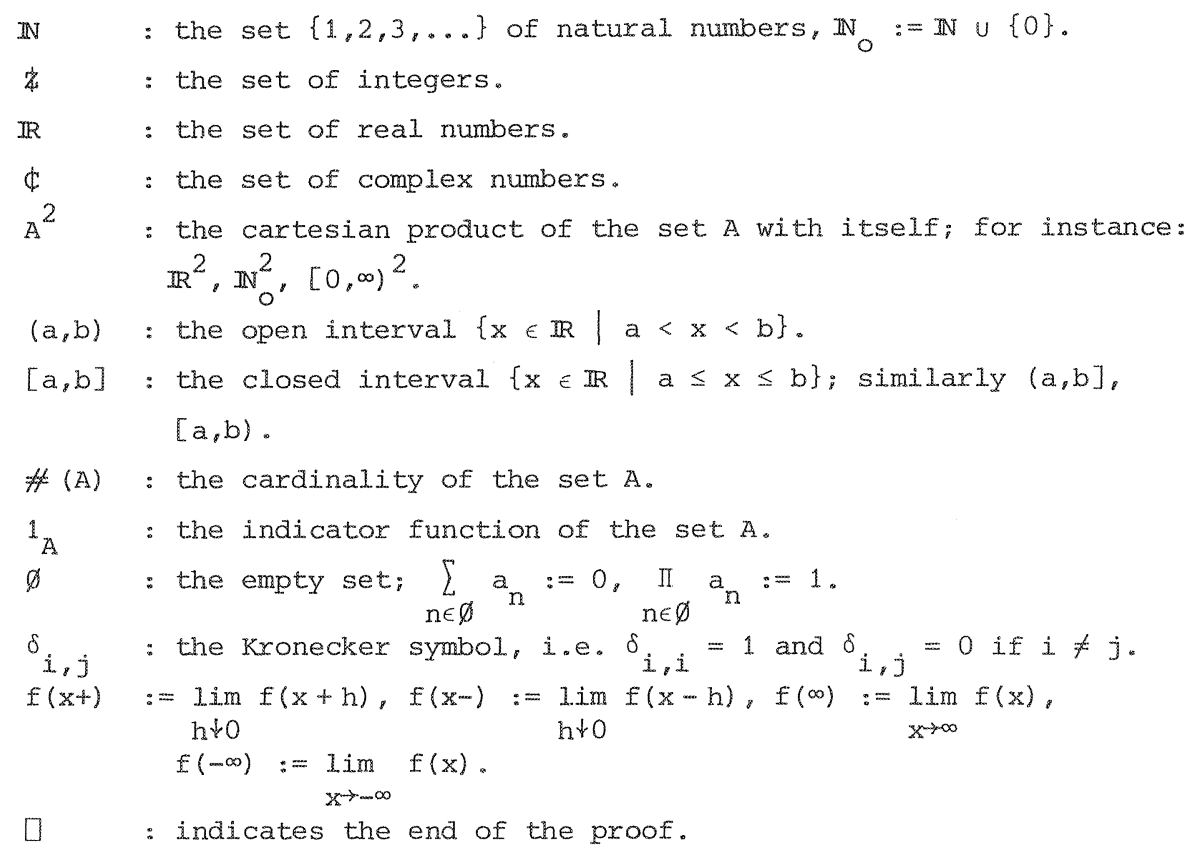

We shall frequently make use of generating functions, Laplace transforms and Fourier transforms; we shall use the following notation for these.

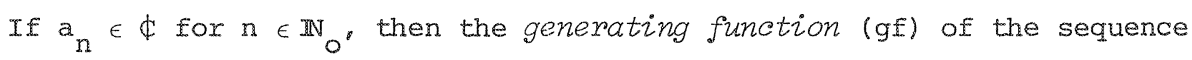
$\left\{a_{n}\right\}_{0}^{\infty}$ is denoted by the corresponding capital, so

$$
A(z)=\sum_{n=0}^{\infty} a_{n} z^{n}
$$

for those $z \in \mathbb{C}$ for which the power series converges. A probability generating function (pgf) is the gf $p$ of a probability distribution $\left\{p_{n}\right\}_{0}^{\infty}$ on $\mathbb{I N}_{0}$. Such distributions will be called lattice distributions; their pgf's are always defined for $|z| \leq 1$.

If $U \neq \equiv$ is a function on $\mathbb{R}$ that is nonnegative, nondecreasing and rightcontinuous, and if $l(U)$, the left extremity of $U$, defined by

$$
\ell(U):=\inf \{x \in \mathbb{R} \mid U(x)>0\},
$$


is finite, then the Laplace-Stieltjes transform (LST) $\bar{U}$ of $U$ is defined by

$$
\hat{U}(\tau):=\int_{(-\infty, \infty)} e^{-\tau X} d U(x)=\int_{[\ell(U), \infty)} e^{-\tau x} d U(x),
$$

for those $\tau \in \mathbb{R}$ for which the integral is finite. If $U$ is a distribution function, then $\hat{\mathrm{U}}$ is called the probability Laplace-Stieltjes transform (PLST) of $U$. The corresponding small letter $u$ will be used for the (probability) density function of $U$ in case of absolute continuity; the ordinary (probability) Laplace transform ( (P) LTS of $u$ is then also denoted by $\hat{U}$, so

$$
\hat{U}(\tau)=\int_{(\ell(U), \infty)} e^{-\tau x} u(x) d x .
$$

Finally, if $U$ is a right-continuous, nondecreasing and bounded function on $\mathbb{R}$ with $U(-\infty)=0$, then the Fourier-mtieltjes transform (FST) $\tilde{U}$ of $U$ is defined by

$$
\tilde{U}(t):=\int_{(-\infty, \infty)} e^{i t x} d U(x)
$$

which exists for all $t \in \mathbb{R}$. Analogous to the LT, we define the ordinary Fourier transform (FT). If $F$ is a distribution function on $\mathbb{R}$, then the FST $\tilde{F}$ is called the characteristic function (chf) of $F$. As $\widetilde{F}$ is continuous and $\widetilde{F}(0)=1$, there exists a neighbourhood of the origin where $\widetilde{F}$ is different from zero. So, the principal branch of the logarithm of $\widetilde{\mathrm{F}}$, denoted by $\log \tilde{\mathrm{F}}(t)$, can be defined uniquely in that neighbourhood.

Besides the abbreviations gf, pgf, LST, PLST, LT, PLT, FST, FT and chf, just introduced, we shall use the following:

$\begin{array}{ll}\text { rv } & \text { random variable } \\ \text { df } & \text { distribution function } \\ \text { pdf } & \text { probability density function } \\ \text { n-div } & \text { n-divisible, n-divisibility } \\ \text { inf div infinitely divisible, infinite divisibility } \\ \text { abs mon absolutely monotone, absolute monotonicity } \\ \text { comp mon completely monotone, complete monotonicity } \\ \text { d } & \text { equal in distribution } \\ \text { iff } & \text { if and only if. }\end{array}$


If $\mathrm{U}$ and $\mathrm{V}$ are nonnegative, nondecreasing and right-continuous functions on $\mathbb{R}$, then the convolution $U * V$ of $U$ and $V$ is defined by

$$
(U * V)(x):=\int_{(-\infty, \infty)} U(x-y) d V(y)=\int_{(-\infty, \infty)} V(x-y) d U(y) \quad(x \in \mathbb{R}) .
$$

which is again a nonnegative, nondecreasing and right-continuous function. For $n \in \mathbb{N}$ the $n$-fold convolution of $U$ with itself is denoted by $U^{* n}$. If $F$ is an inf div df, then for $\gamma>0 \mathrm{~F}^{* \gamma}$ denotes the df with chf $\widetilde{F}^{\gamma}$. If $\left\{a_{n}\right\}_{0}^{\infty}$ is a sequence with $a_{0} \neq 0$ and with gf $A$, then for $\gamma>0$ the sequence $\left\{a_{n}^{* \gamma}\right\}_{0}^{\infty}$ is defined by its gf as follows:

$$
\sum_{n=0}^{\infty} a_{n}^{* \gamma} z^{n}=A(z)^{\gamma}
$$

Unless stated otherwise, throughout this monograph we only consider proba-m bility distributions on $\mathbb{I R}$ that are not concentrated at zero. For instance, as in case of a lattice distribution $\left\{p_{n}\right\}_{0}^{\infty}$ we often take $p_{0}>0$ (cf. section 5), it is then tacitly assumed that $0<p_{0}<1$.

If $\left\{p_{n}\right\}_{0}^{\infty}$ is a lattice distribution in some class $C$ with pgf $p$, then we shall also say that $P \in C$. Similar conventions hold for $\hat{F}$ and $\tilde{F}$.

Finally, if $C$ is a class of probability distributions, then a family $\left(C_{t} \mid t \in T\right)$ of subclasses of $C$ is said to define a classification of $C$, if $T$ can be totally ordered in such a way that the classes $C_{t}$ are nondecreasing in the ordering of $T$. It follows that for $t_{1}<t_{2}<\ldots<t_{n}$ the clas$\operatorname{ses} C_{t_{1}}, C_{t_{2}} \backslash C_{t_{1}}, \ldots, C_{t_{n}} \backslash C_{t_{n-1}}, C \backslash C_{t_{n}}$ form a partition of $C$.

\subsection{Absolute and complete monotonicity}

In the sequel we shall characterize several classes of probability distributions by making use of the concepts of absolute and complete monotonicity (cf. Widder (1946), ch. IV and Fellex (1971), ch. VII and XIII). Since we only need absolute monotonicity on intervals of the form $[0, \rho)$ and complete monotonicity on $(0, \infty)$, we usually do not mention these intervals. We start with considering absolutely monotone functions.

DEFINITION 1.3.1. A function $R$ is said to be absolutely monotone on $[0, p)$ if it is continuous there and possesses derivatives of all orders on $(0, p)$ with 


$$
\left(\frac{d}{d z}\right)_{R(z)} \geq 0 \quad\left(n \in \mathbb{N}_{0} ; 0<z<\rho\right) .
$$

$R$ is said to be absolutely monotone (abs mon) if there exists $p>0$ such that $R$ is abs mon on $[0, \rho)$.

When proving the abs mon of a function, we shall often utilize the following characterization (cf. Widder (1946), ch. IV).

THEOREM 1.3.2. A function $R$ is abs mon on $[0, \rho)$ iff there exist $x_{n} \geq 0$ ( $\mathrm{n} \in \mathbb{N}_{\mathrm{O}}$ ) such that

$$
\text { (1.3.1) } R(z)=\sum_{n=0}^{\infty} r_{n} z^{n} \quad(0 \leq z<\rho) \text {; }
$$

in this case the quantities $r_{n}$ are given by $r_{n}=R^{(n)}(0+) / n:\left(n \in \mathbb{N}_{0}\right)$.

Thus, an abs mon function $R$ on $[0, \rho)$ can be extended analytically to the aisk $|z|<\rho$.

There exists a number of simple properties of abs mon functions that we shall use in the sequel without further comment; the following lemma contains some of them.

LEMMA 1.3 .3$.

(i) $R$ is abs mon iff $R(0) \geq 0$ and $R^{\prime \prime}(z)$ is abs mon.

(ii) If $R$ is abs mon, then so are $R(\alpha, z)$ and $R(z)-R(\alpha z)$ for all $\alpha \in(0,1)$.

(iii) If $R$ and $S$ are abs mon, then so are $R(z)+S(z)$ and $R(z) S(z)$.

(iv) If $R_{n}$ is abs mon on $[0, \rho)(n \in \mathbb{N})$ and if $R(z)=\lim _{n \rightarrow \infty}(z)$ exists for $z \in[0, \rho)$, then $R$ is abs mon on $[0, \rho)$.

(v) If $R$ is abs mon on $[0, \rho)$ and if $S$ is abs mon on $[0, \sigma)$ with $S(z)<\rho$ $(0 \leq z<\sigma)$, then $R(S(z))$ is abs mon on $[0, \sigma)$. For instance:

(a) If $S$ is abs mon, then $\exp [S(z)]$ is abs mon;

(b) If $S$ is abs mon with $S(z)<1$ in some interval $[0, \sigma)$, then $\{1-\mathrm{S}(z)\}^{-1}$ is abs mon.

The following lemma, and simple extensions of it, will be used particularly in chapter 3.

LEMMA 1.3.4. Let $P$ be a pgf with $P(0)>0$, and let $Q$ be a pgf. If the function $R$, defined by 


$$
R(z):=Q(z) / P(z)
$$

is abs mon, then $R$ coincides, at least in $|z| \leq 1$, with a pgfo

PROOF. AS $R$ is abs mon, by theorem 1.3 .2 there exist $\rho>0$ and $x_{n} \geq 0$ ( $\mathrm{n} \in \mathbb{N}_{\mathrm{o}}$ ) such that

$$
(1.3 .2) \quad R(z)=\sum_{n=0}^{\infty} r_{n} z^{n} \quad(|z|<\rho) .
$$

Since $P(0)>0$, we may assume that $P(z) \neq 0$ for $|z|<\rho$, and hence, if $P$ and $Q$ are the pgf's of $\left\{p_{n}\right\}_{0}^{\infty}$ and $\left\{q_{n}\right\}_{0}^{\infty}$, respectively, then

$$
q_{n}=\sum_{k=0}^{n} r_{k} p_{n-k} \quad\left(n \in \mathbb{N}_{0}\right)
$$

Summing over $n$ we get

$$
1=\sum_{n=0}^{\infty} \sum_{k=0}^{n} r_{k} p_{n-k}=\sum_{k=0}^{\infty} r_{k} \sum_{n=k}^{\infty} p_{n-k}=\sum_{k=0}^{\infty} r_{k} .
$$

i.e. $\left\{x_{n}\right\}_{0}^{\infty}$ is a probability distribution. Now, let $A$ denote the set of poles of $R$ in $|z| \leq 1$, then, as $P$ has finitely many zeros in $|z| \leq 1$, we have $\#(A)<\infty$, while by analytic continuation we see that the equality in $(1.3 .2)$ holds in $\{|z| \leq 1\} \backslash A$. However, since $\Sigma r_{n} z^{n}$ is bounded in $|z| \leq 1$, we necessarily have $A=\emptyset$, and the lemma is proved.

Finally, we state the continuity theorem for pgf"s, which we shall need several times. It can be found in Feller (1968), ch. XI.

THEOREM 1.3.5. Suppose that for every $n \in \mathbb{N}$ the sequence $\left\{\underline{p}_{k}(n)\right\}_{k=0}^{\infty}$ is a probability distribution with pgf $\mathrm{P}_{\mathrm{n}}$ '

(i) If $p_{k}:=\lim _{n \rightarrow \infty} p_{k}(n)$ exists for all $k \in \mathbb{N}_{0^{\prime}}$ then $P(z):=\lim _{n \rightarrow \infty} P_{n}(z)$ exists for all $z \in[0,1]$, while

(1.3.3) $\quad \mathrm{P}(\mathrm{z})=\sum_{\mathrm{k}=0}^{\infty} \mathrm{p}_{\mathrm{k}} \mathrm{z}^{\mathrm{k}} \quad(0 \leq z<1)$.

If in addition $\left\{p_{k}\right\}_{0}^{\infty}$ is a probability distribution, then $P$ is the pgf of $\left\{\mathrm{p}_{\mathrm{k}}\right\}$ (in fact, as is easily shown, $P(z):=\lim _{n \rightarrow \infty} \mathrm{P}_{n}(z)$ exists for $|z| \leq 1$ and (1.3.3) holds for $|z| \leq 1)$. 
(ii) If $P(z):=\lim _{n \rightarrow \infty} P_{n}(z)$ exists for all $z \in(0,1)$, then $p_{k}:=\lim _{n \rightarrow \infty} p_{k}(n)$ exists for all $k \in \mathbb{N}_{0}$, while (1.3.3) holds. If in addition $P$ is leftcontinuous in $z=1$, then $\left\{p_{k}\right\}_{0}^{\infty}$ is a probability distribution with pgf $P$.

Next, we consider completely monotone functions.

DEFINITION 1.3.6. A function $\varphi$ on $(0, \infty)$ is said to be completely monotone (comp mon) if $\varphi$ possesses derivatives of all orders on $(0, \infty)$ with

$$
(-1)^{n}\left(\frac{d}{d \tau}\right)^{n} \varphi(\tau) \geq 0 \quad\left(n \in \mathbb{N}_{0} ; \tau>0\right) .
$$

The comp mon functions can be represented as LST'S; this result is known as Bernstein's theorem (see e.g. Feller (1971), ch. XIII).

THEOREM 1.3.7. A function $\varphi$ on $(0, \infty)$ is comp mon iff there exists a nonnegative, right-continuous and nondecreasing function $U$ with $\ell(U) \geq 0$ such that $\varphi=\hat{U}, i . e$. such that

$$
\varphi(\tau)=\int_{[0, \infty)} e^{-\tau x} d U(x) \quad(\tau>0) .
$$

In the following lemma we sumarize the principal properties of comp mon functions (cf. Feller (1971), ch. XIII); they will be used without further comment.

LEMMA 1.3 .8 .

(i) $\varphi$ is comp mon iff $-\varphi^{\prime \prime}(\tau)$ is comp mon and $\varphi(\infty) \geq 0$.

(ii) If $\varphi$ is comp mon, then so are $\varphi(\lambda \tau), \varphi(\tau+\lambda)$ and $\varphi(\tau)-\varphi(\tau+\lambda)$ for all $\lambda>0$.

(iii) If $\varphi$ and $\psi$ are comp mon, then so are $\varphi+\psi$ and $\varphi \psi$.

(iv) If $\varphi_{n}$ is comp mon $(n \in \mathbb{N})$ and if $\varphi(\tau):=\lim _{n \rightarrow \infty} \varphi_{n}(\tau)$ exists for $\tau>0$, then $\varphi$ is comp mon.

(v) If $R$ is abs mon on $[0, \rho)$ and if $\varphi$ is comp mon with $\varphi(\tau)<\rho(\tau>0)$, then $R(\varphi(\tau))$ is comp mon. For instance, if $\varphi$ is comp mon, then $\exp [\varphi(\tau)]$ is comp mon, and if in addition $\varphi(\tau)<1(\tau>0)$, then $\{1-\varphi(\tau)\}^{-1}$ is comp mon. 
(vi) If $\varphi$ and $\psi^{\prime}$ are comp mon and if $\psi(0+) \geq 0$, then $\varphi(\psi(\tau))$ is comp mon. For instance, if $\psi^{3}$ is comp mon and $\psi(0+) \geq 0$, then $\exp [-\psi(\tau)]$ and $\{1+\psi(\tau)\}^{-1}$ are comp mon.

We also mention two relations between the LST Û (comp mon if $\ell(U) \geq 0$ ) and the function $U$, which we shall use repeatedly.

LEMMA 1.3.9. Let $U \neq \equiv$ be a nonnegative, right-continuous and nondecreasing function on $\mathbb{R}$ with $\ell(U)>-\infty$ and such that $\tilde{U}(\tau)$ exists for $\tau>\tau_{0}$. Then

$$
(1.3 .4) \quad U(\ell(U))=\lim _{\tau \rightarrow \infty} \hat{U}(\tau) e^{\ell(U) \tau}
$$

and, if $\ell(U) \geq 0$,

$(1.3 .5) \quad U(0)=\lim _{\tau \rightarrow \infty} \hat{U}(\tau)$

If $\tau_{0} \leq 0$, then also

$$
(1.3 .6) \quad \mathrm{U}(\infty)=\lim _{\tau \downarrow 0} \hat{U}(\tau)
$$

PROOF. In view of the definition of $\hat{U}$ we can write

$$
\tilde{U}(\tau) e^{\ell(U) \tau}=U(\ell(U))+\int_{(\ell(U), \infty)} e^{-\tau(x-\ell(U))} d U(x),
$$

from which (1.3.4) follows by the dominated convergence theorem. Similarly we obtain (1.3.5). Finally, applying the monotone convergence theorem, we see that

$$
\lim _{\tau \downarrow 0} \tilde{U}(\tau)=\int_{[\ell(U), \infty)} d U(x)=\lim _{x \rightarrow \infty} U(x)=U(\infty) .
$$

Finally, we give a definition of comp mon for sequences and a representation of such sequences, which is due to Hausdorff (cf. Feller (1971), ch. VII).

DEFINITION 1.3.10. A sequence $\left\{a_{n}\right\}_{0}^{\infty}$ of real numbers is called comp mon if

$$
(-1)^{n} \Delta^{n} a_{k} \geq 0 \quad\left(n, k \in \mathbb{M}_{0}\right)
$$

where $\Delta a_{k}:=a_{k+1}-a_{k} \Delta^{\circ} a_{k}:=a_{k}$ and $\Delta^{n}:=\Delta\left(\Delta^{n-1}\right)$. 
THEOREM 1.3.11. A sequence $\left\{a_{n}\right\}_{0}^{\infty}$ is comp mon iff there exists a finite measure $v$ on $[0,1]$ such that

$$
(1.3 .7) \quad a_{n}=\int_{[0,1]} x^{n} v(d x) \quad\left(n \in \mathbb{N}_{0}\right) .
$$

\subsection{Definition and basic properties of infinitely divisible distributions} on $\mathbb{R}$

The concept of infinite divisibility can be introduced as follows.

DEFINITION 1.4.1. For $n \in \mathbb{N}$ a $r v x$ is said to be $n$-divisible ( $n$-div) if there exist independent and identically distributed $r v^{\prime} s x_{n, 1} \ldots, x_{n, n}$ such that

$$
x \stackrel{d}{=} x_{n, 1}+\ldots+x_{n, n}
$$

A $r v x$ is said to be infinitely divisible (inf div) if $x$ is n-div for all $\mathrm{n} \in \mathbb{N}$.

In fact, inf div is a property of the distribution of $\mathrm{x}$; therefore we call the df, pdf, chf, etc., corresponding to an inf div $r v x$, inf div too. Thus, a chf $\tilde{F}$ is inf div iff for every $n \in \mathbb{N}$ there exists a df $F_{n}$ such that

$$
\widetilde{F}(t)=\left\{\tilde{F}_{n}(t)\right\}^{n} \quad(t \in \mathbb{R})
$$

Next we list a number of basic properties of inf div distributions that we need in the following chapters: they can be found in Lukacs (1970). The first three of them have obvious analogues for pgf's and PLST"S.

THEOREM 1.4.2. If $\widetilde{\mathrm{F}}$ and $\widetilde{\mathrm{G}}$ are inf div chf's, then $\widetilde{\mathrm{F}} \widetilde{G}$ is an inf div chf.

THEOREM 1.4.3 (Closure theorem). A chf which is the limit of a sequence of inf div chf's, is inf div.

THEOREM 1.4.4. A nonvanishing chf $\widetilde{\mathrm{F}}$ is inf div iff $\widetilde{\mathrm{F}}^{\gamma}$ is a chf for all $\gamma>0$ (or for all $\gamma=1 / n, n \in \mathbb{N}$, or for all $\gamma=2^{-n}, n \in \mathbb{N}$ ).

THEOREM 1.4.5. An inf div chf has no real zeros. 
THEOREM 1.4.6. If the $\mathrm{rV} \mathrm{x}$ is nondegenerate and bounded, then $\mathrm{x}$ is not inf div.

THEOREM 1.4.7 (Lêvy canonical representation). A function $\varphi$ on $\mathbb{R}$ is an inf div chf iff $\varphi$ has the form

$$
\text { (1.4.1) } \varphi(t)=\exp \left[i t a-\frac{1}{2} \sigma^{2} t^{2}+\int_{\mathbb{R} \backslash\{0\}}\left\{e^{i t x}-1-\frac{i t x}{1+x^{2}}\right\} d M(x)\right] \quad(t \in \mathbb{R}) \text {, }
$$

where $a \in \mathbb{R}, \sigma^{2} \geq 0$ and $M$ is a right-continuous function on $\mathbb{R} \backslash\{0\}$ with the following properties: $\mathbb{M}$ is nondecreasing on $(-\infty, 0)$ and on $(0, \infty), \mathbb{M}(-\infty)=$

$=M(\infty)=0$, and

$$
\int_{(-1,1) \backslash\{0\}} x^{2} d M(x)<\infty
$$

REMARK 1.4.8. If a chf $\tilde{F}$ has a representation of the form (1.4.1), where $\mathbb{M}$ violates the monotonicity condition of the theorem, then $F$ is not inf div.

The canonical representation (1.4.1) can be somewhat modified to obtain other well known representations. For instance, in the Lévy-Khintchine representation an inf div chf $\tilde{\mathrm{F}}$ has the form

$$
\text { (1.4.3) } \tilde{\mathrm{F}}(t)=\exp \left[i \mathrm{ta}+\int_{(-\infty, \infty)}\left\{e^{i t x}-1-\frac{i t x}{1+x^{2}}\right\} \frac{1+x^{2}}{x^{2}} d \theta(x)\right] \quad(t \in \mathbb{R}) .
$$

where $\mathrm{a} \in \mathbb{R}$ and $\theta$ is a right-continuous, nondecreasing and bounded function on $\mathbb{R}$ with $\theta(-\infty)=0$ (for $x=0$ the integrand is defined by continuity: $-\frac{1}{2} t^{2}$ ). The canonical representations $(1.4 .1)$ and $(1.4 .3)$ are generalizations of the following representation, due to Kolmogorov, which is valid only for chf's of inf div distributions with finite second moment:

$$
\text { (1.4.4) } \tilde{F}(t)=\exp \left[i t a+\int_{(-\infty, \infty)}\left\{e^{i . t x}-1-i t x\right\} \frac{1}{x^{2}} d K(x)\right] \quad(t \in \mathbb{R}) .
$$

where $\mathrm{a} \in \mathbb{R}$ and $\mathrm{K}$ is a right-continuous, nondecreasing and bounded function on IR with $\mathrm{K}(-\infty)=0$. We prefer the Lévy canonical representation, as it has the clearest relations with the canonical representations known for inf div distributions on $[0, \infty)$ and on $\mathbb{N}_{0}$, which are special cases; this will be clarified in section 7 . 
Simple examples of inf div distributions are provided by the degenerate, Poisson, negative-binomial (and hence geometric), gamma (and hence exponential), normal and Cauchy distributions; their inf div is easily verified from their chf's. Considerably harder to prove is the inf div of the lognormal and the Student distributions; this has recently been done by Thorin (1977) and Grosswald (1976), respectively.

There are several methods to construct new inf div distributions from given ones; the best known are convolution, compounding and mixing. As an example of the method of mixing we state the following theorem of Feller (1971), ch. XVII (see also steutel (1970)), and we note that in section 6 mixtures of exponential distributions are considered.

THEOREM 1.4.9. If $G$ and $H$ are inf div df's on $[0, \infty)$ and $\mathbb{R}$, respectively, then

$(1.4 .5) \quad \hat{G}(-\log \tilde{H}(t))=\int_{[0, \infty)} \tilde{H}(t)^{x} \mathrm{dG}(x) \quad(t \in \mathbb{I R})$

is an inf div chfo

COROLTARY 1.4.10. If $G$ is an inf div df on $[0, \infty)$, then the following mixture of normal chf's is inf div:

(1.4.6) $\int_{[0, \infty)} \exp \left[-t^{2} x\right] \mathrm{dG}(x) \quad(t \in \mathbb{R})$.

Finally, we pay some attention to compound distributions. Here we use the terminology of Feller; such distributions are also called generalized distributions by some authors (cf. Gurland (1957) and Johnson \& Kotz (1969)).

DEFINITION 1.4.11. A probability distribution is called a compound distribution if its chf $\tilde{F}$ can be written in the form

$(1.4 .7) \quad \widetilde{F}(t)=P(\widetilde{G}(t)) \quad(t \in \mathbb{I R})$. where $P$ is a pgf and $G$ is a df.

A xry $X$ with chf $\tilde{F}$ given by $(1.4 .7)$ can be represented as

$$
\mathrm{X} \stackrel{\mathrm{d}}{=} \mathrm{Y}_{1}+\mathrm{Y}_{2}+\ldots+\mathrm{Y}_{\mathrm{N}} \text {. }
$$

where $N, Y_{1}, Y_{2}, \ldots$ are independent, $N$ has a lattice distribution with pgf $P$ and $Y_{1}, Y_{2}, \ldots$ are identically distributed with df $G$. 
EXAMPLE 1.4 .12 .

(i) A compound Poisson chf $\tilde{F}$ is a chf of the form

$(1.4 .8) \quad \tilde{F}(t)=\exp [\mu(\tilde{G}(t)-1)] \quad(t \in \mathbb{R})$,

where $\mu>0$ and $G$ is a df.

(ii) A compound geometric chf $\tilde{\mathrm{F}}$ is a chf of the form

$$
\begin{gathered}
\text { (1.4.9) } \tilde{F}(t)=\frac{1-p}{1-p \tilde{G}(t)} \quad(t \in \mathbb{R}), \\
\text { where } 0<p<1 \text { and } G \text { is a df. }
\end{gathered}
$$

REMARK 1.4.13. For a chf $\widetilde{F} \not 1$ of the form $(1.4 .8)$ or $(1.4 .9)$ it is possible to choose the df $G$ in such a way that $G$ is continuous at zero. We shall always do so: the representations $(1.4 .8)$ and $(1.4 .9)$ are then unique, and we will refer to them as compound-Poisson- $(\mu, G)$ and compound-geometric-$(p, G)$ distributions, respectively.

The compound Poisson and the compound geometric (more general: compound negative-binomial) distributions are known to be inf div (cf. Lukacs (1970), ch. 5). In fact, this is a consequence of the following property of compound distributions .

LEMMA 1.4.14. If $P$ is an inf div pgf with $P(0)>0$, then for all df's $G$ the compound chf $\widetilde{F}(t)=P(\widetilde{G}(t))$ is compound Poisson and hence inf div.

PROOF. As we shall see in theorem 1.5.1, if $\mathrm{P}$ is an inf div pgf with $\mathrm{P}(0)>0$, then $P$ is compound Poisson, so

$$
P(z)=\exp [\mu(Q(z)-1)] \quad(|z| \leq 1),
$$

with $\mu>0$ and $Q$ is a pgf with $Q(0)=0$. It follows that

$$
\tilde{F}(t)=P(\tilde{G}(t))=\exp [\mu(Q(\tilde{G}(t))-1)] .
$$

i.e. $F$ is compound-Poisson- $(\mu, H)$, with $\tilde{H}(t):=Q(\tilde{G}(t))$.

In sections 5 and 6 compound distributions on $\mathbb{N}_{0}$ and on $[0, \infty)$ will be considered in more detail. We conclude this section with De Finetti's observation, that every inf div distribution can be obtained as the weak limit of compound Poisson distributions (cf. Lukacs (1970), ch.5). 
THEOREM 1.4.15. A chf $\tilde{F}$ is inf div iff $\tilde{\mathrm{F}}$ has the form

$(1.4 .10) \quad \tilde{F}(t)=\lim _{n \rightarrow \infty} \exp \left[\mu_{n}\left(\tilde{G}_{n}(t)-1\right)\right] \quad(t \in \mathbb{R})$,

where $\mu_{n}>0$ and $G_{n}$ is a df $(n \in \mathbb{N})$. In this case we may take $\mu_{n}=n$ and $G_{n}=F^{* 1 / n}(n \in \mathbb{N})$.

\subsection{Infinitely divisible lattice distributions}

Let $\left\{p_{n}\right\}_{0}^{\infty}$ be a lattice distribution, i.e. a probability distribution on $\mathbb{N}$ 。 When investigating the inf div of $\left\{p_{n}\right\}$, we shall always require that

$0<p_{0}<1$; the condition " $p_{0}>0$ " ensures that, in case of inf div of $\left\{p_{n}\right\}$, the distribution $\left\{\mathrm{p}_{\mathrm{n}}^{* 1 / \mathrm{k}}\right\}_{n=0}^{\infty}$ (with $\operatorname{pgf} \mathrm{P}(\mathrm{z})^{1 / \mathrm{k}}$ ) is again a distribution on $\mathbb{N}_{0}$. It is not an essential restriction: for all $\gamma \in \mathbb{R}, P\left(e^{i t}\right)$ is an inf div chf iff $e^{i t \gamma} P\left(e^{i t}\right)$ is an inf div chf. Further we note that $\log P(z)$ and $P(z)^{\gamma}(\gamma \in \mathbb{R})$ are always uniquely defined in a neighbourhood of zero if $p_{0}>0$.

For an inf div pgf $\mathrm{P}$ we have the following representation theorem (cf. Fellex (1968), ch. XII).

THEOREM 1.5.1. A pgf $\mathrm{P}$, with $0<\mathrm{P}(0)<1$, is inf div iff $\mathrm{P}$ is compound Poisson, $i . e$. iff $\mathrm{P}$ has the form

(1.5.1) $\quad \mathrm{P}(z)=\exp [\mu(2(z)-1)] \quad(|z| \leq 1)$.

where $\mu>0$ and $Q$ is a pgf with $Q(0)=0$. The representation $(\mu, Q)$ is unique.

COROLLARY 1.5.2. An inf div pgf $P$ with $P(0)>0$ has no zeros in the closed unit disk.

Feller (1968) reformulates theorem 1.5.1 to obtain a criterion for inf div. We shall now do so in a slightly different way, using the concept of absolute monotonicity (cf, definition 1.3.1). Additionally we obtain a slightly different representation for inf div pgf's, which is sometimes more convenient.

THEOREM 1.5.3. A pgf $P$, with $0 \leqslant P(0)<1$, is inf div iff the function $R_{1}$, defined by 
(1.5.2) $\quad \mathrm{R}_{1}(z):=\mathrm{P}^{\mathrm{y}}(z) / \mathrm{P}(z)$

is abs mon, or, equivalently, iff there exist nonnegative quantities $r_{n}(1)$

( $n \in \mathbb{N}_{0}$ ) satisfying

(1.5.3) $\sum_{n=0}^{\infty} \frac{r_{n}(1)}{n+1}<\infty$,

such that $P$ has the form

(1.5.4) $\quad P(z)=\exp \left[\sum_{n=0}^{\infty} \frac{r_{n}(1)}{n+1}\left(z^{n+1}-1\right)\right] \quad(|z| \leq 1)$.

PROOF. If $\mathrm{P}$ is inf div with $0<\mathrm{P}(0)<1$, then $\mathrm{P}$ has the form $(1.5 .1)$ and hence $R_{1}(z)=\mu Q^{\prime}(z)$ is abs mon.

Next, let $R_{1}$ be abs mon. Then there exist $\rho>0$ and $r_{n}(1) \geq 0\left(n \in \mathbb{N}_{0}\right)$ such that

(1.5.5) $\quad P^{\prime}(z) / P(z)=\sum_{n=0}^{\infty} x_{n}(1) z^{n} \quad(|z|<\rho)$.

Integrating this equation from 0 to $z(|z|<\rho)$, we obtain

(1.5.6) $\quad \log \{P(z) / P(0)\}=\sum_{n=0}^{\infty} \frac{r_{n}(1)}{n+1} z^{n+1} \quad(|z|<\rho)$.

From $(1.5 .5)$ we get the following relations:

$$
(n+1) p_{n+1}=\sum_{k=0}^{n} p_{k} r_{n-k}(1) \quad\left(n \in \mathbb{N}_{0}\right),
$$

from which by the nonnegativity of the $r_{n}(1)$ "s it can be shown (cf. lemma 1.5.6) that $(1.5 .3)$ holds. Hence the power series in $(1.5 .6)$ is convergent for $|z| \leq 1$, and by analytic continuation it follows that the equality in (1.5.6) holds for $|z| \leq 1$. Taking $z=1$, one sees that

$(1.5 .7)-\log \mathrm{P}(0)=\sum_{n=0}^{\infty} \frac{r_{n}(1)}{n+1}$

hence $P$ takes the form $(1.5 .4)$.

Finally, if $P$ has the form $(1.5 .4)$ with nonnegative $r_{n}(1)$ 's satisfying (1.5.3), then defining 
(1.5.8) $\mu:=\sum_{n=0}^{\infty} \frac{r_{n}(1)}{n+1}$ and $Q(z):=\frac{1}{\mu} \sum_{n=0}^{\infty} \frac{r_{n}(1)}{n+1} z^{n+1} \quad(|z| \leq 1)$.

we see that $\mathrm{p}$ takes the form $(1.5 .1)$ and hence is inf div.

REMARK 1.5.4. To some quantities we add an index 1 or 0 in order to fit them in the more general notation of the next chapter. For instance: $R_{1}, r_{n}(1)$, and, presently, $R_{0}$ and $x_{n}(0)$.

The sequence $\left\{r_{n}(1)\right\}$ from the preceding theorem is uniquely determined by $P$, its gf $R_{1}$ satisfies (1.5.2). Therefore $\left\{x_{n}(1)\right\}$ is called the canonical sequence of the inf div pgf $P$; its relation with the Levy canonical representation $\left(a, \sigma^{2}, M\right)$ for $P\left(e^{i t}\right)$ will be shown in section 7.

From theorem 1.5.3 one easily verifies the following theorem, due to Katti (1967), which gives a characterization of the inf div lattice distributions in terms of the $p_{n}^{\prime} ' s$ themselves.

COROLIARY 1.5.5. A lattice distribution $\left\{\mathrm{p}_{\mathrm{n}}\right\}_{0}^{\infty}$ with $0<\mathrm{p}_{0}<1$ is inf div iff there exist nonnegative quantities $r_{n}(1)\left(n \in \mathbb{N}_{0}\right)$ such that

(1.5.9) $\quad(n+1) p_{n+1}=\sum_{k=0}^{n} p_{k} x_{n-k}(1) \quad\left(n \in \mathbb{N}_{0}\right)$

It is useful to consider the recurrence relations $(1.5 .9)$ in some more de-tail.

LEMMA 1.5 .6 .

(i) If $\left\{p_{n}\right\}_{0}^{\infty}$ is a lattice distribution with $p_{0}>0$, then there exists a unique sequence $\left\{r_{n}(1)\right\}_{0}^{\infty}$ satisfying (1.5.9); its gf $R_{1}$ has a positive radius of convergence, while for $|z|$ sufficiently small

$(1.5 .10) \quad R_{1}(z)=P^{3}(z) / P(z)$.

If, in addition, all $r_{n}(1)^{\prime} s$ are nonnegative, then necessarily

(1.5.11) $\sum_{n=0}^{\infty} \frac{x_{n}(1)}{n+1}<\infty$.

(ii) If $\left\{x_{n}(1)\right\}_{0}^{\infty}$ is a sequence of nonnegative numbers satisfying (1.5.11), then there exists a unique lattice distribution $\left\{\mathrm{p}_{\mathrm{n}}\right\}_{0}^{\infty}$, with $\mathrm{p}_{0}>0$, satisfying $(1.5 .9)$. 
PROOF .

(i) Evidently, the first $n+1$ equations in (1.5.9) determine $x_{0}(1), r_{1}(1), \ldots$ $\ldots, r_{n}(1)$. As $p_{0}>0$, the function $R_{1}$, defined by $(1.5 .10)$, is analytic in a neighbourhood of zero, and therefore has a power-series expansion with coefficients $r_{0}, r_{1}, \ldots$, say. But from $(1.5 .10)$ it follows that the $x_{n}^{\prime}$ 's satisfy $(1.5 .9)$, so $x_{n}=r_{n}(1) \quad\left(n \in \mathbb{N}_{0}\right)$, and $R_{1}$ is the gf of the sequence $\left\{r_{n}(1)\right\}$.

If, in addition, all $x_{n}(1)$ 's axe nonnegative, then we can write

$$
\begin{aligned}
1-p_{0} & =\sum_{n=0}^{\infty} p_{n+1}=\sum_{n=0}^{\infty} \frac{1}{n+1} \sum_{k=0}^{n} p_{k} x_{n-k}(1)= \\
& =\sum_{k=0}^{\infty} p_{k} \sum_{n=0}^{\infty} \frac{x_{n}(1)}{n+1+k} \geq p_{0} \sum_{n=0}^{\infty} \frac{r_{n}(1)}{n+1}
\end{aligned}
$$

and hence

$$
\sum_{n=0}^{\infty} \frac{r_{n}(1)}{n+1} \leq \frac{1-p_{0}}{p_{0}}<\infty .
$$

(ii) Clearly, there exists at most one probability distribution $\left\{\mathrm{p}_{n}\right\}_{0}^{\infty}$ with $p_{0}>0$, satisfying $(1.5 .9)$ for given $r_{n}(1)$. Now, if $r_{n}(1) \geq 0\left(n \in \mathbb{N}_{0}\right)$ and if (1.5.11) holds, then $i t$ is seen that the function $P$ defined by $(1.5 .4)$ is abs mon with $P(1)=1, i . e . P$ is a pgf. It follows that if $R_{1}$ is the $g f$ of $\left\{r_{n}(1)\right\}$, then $P$ satisfies $(1.5 .10), i . e$. the coefficients $p_{n}$ of $P$ satisfy $(1.5 .9)$.

The following result about zeros of an inf div $\left\{p_{n}\right\}_{0}^{\infty}$ can be derived from corollary 1.5 .5 (cf. Steutel (1970)).

THEOREM 1.5.7. If $\left\{p_{n}\right\}$ is an inf div lattice distribution with $0<p_{0}<1$, then for all $n \in \mathbb{N}_{0}$ and all $k \in \mathbb{N}_{0}$ the following implication holds:

$$
\left[p_{n}>0 \text { and } p_{k}>0\right] \Rightarrow p_{n+k}>0 \text {. }
$$

Consequently, if $p_{1}>0$ then $p_{n}>0$ for all $n \in \mathbb{N}_{0}$.

Next we turn to the compound geometric lattice distributions, i.e. (cf. example $1.4 .12(i i)$ ) distributions with pgf $P$ of the form

$$
(1.5 .12) \quad P(z)=\frac{1-p}{1-p Q(z)} \quad(|z| \leq 1)
$$

where $0<p<1$ and $Q$ is a pgf with $Q(0)=0$. These distributions are inf 
div (cf. Lemma 1.4.14) and have properties similar to those of the compound poisson lattice distributions.

THEOREM 1.5.8. A pgf $P$ with $0<P(0)<1$ is compound geometric iff the function $R_{0}$, defined by

$(1.5 .13) \quad R_{0}(z):=\frac{1}{z}\{1-P(0) / P(z)\}$

is abs mon.

PROOF. The necessity of the condition immediately follows from (1.5.12). So, let $R_{0}$ be abs mon, $i_{0} e$. there exist $\rho>0$ and $r_{n}(0) \geq 0$ ( $n \in \mathbb{N}_{0}$ ) such that $R_{0}$ has a power-series representation for $|z|<\rho$ with coefficients $r_{n}(0)$. Then from (1.5.13) it follows that

$$
(1.5 .14) \quad P(z) / P(0)=\left\{1-\sum_{n=0}^{\infty} r_{n}(0) z^{n+1}\right\}^{-1} \quad(|z|<\rho)
$$

and that

$$
p_{n+1}=\sum_{k=0}^{n} p_{k} x_{n-k}(0) \quad\left(n \in \mathbb{N}_{0}\right)
$$

From these relations it can be shown (cf. lemma 1.5.10) that $\sum_{n=0}^{\infty} r_{n}(0)<1$, and hence the right-hand side of $(1.5 .14)$ is an analytic function on $|z| \leq 1$. It follows that the equality in (1.5.14) holds for $|z| \leq 1$. Taking $z=1$ we see that

$(1.5 .15) \quad P(0)=1-\sum_{n=0}^{\infty} r_{n}(0)$

and hence $\mathrm{P}$ takes the form $(1.5 .12)$ i.f we define

$(1.5 .16) \quad p:=\sum_{n=0}^{\infty} x_{n}(0)$ and $Q(z):=\frac{1}{p} \sum_{n=0}^{\infty} x_{n}(0) z^{n+1} \quad(|z| \leq 1)$.

From theorem 1.5 .8 one obtains the following analogue of corollary 1.5 .5 (cf. Steutel (1970)).

COROLLARY 1.5.9. A lattice distribution $\left\{p_{n}\right\}_{0}^{\infty}$ with $0<p_{0}<1$ is compound geometric iff there exist nonnegative quantities $x_{n}(0)\left(n \in \mathbb{N}_{0}\right)$ such that $(1.5 .17) \quad p_{n+1}=\sum_{k=0}^{n} p_{k} x_{n-k}(0) \quad\left(n \in \mathbb{N}_{0}\right)$. 
The following lemma is the analogue of lemma 1.5 .6 for the recurrence relations $(1.5 .17)$.

LEMMA 1.5 .10 .

(i) If $\left\{\mathrm{p}_{\mathrm{n}}\right\}_{0}^{\infty}$ is a lattice distribution with $\mathrm{p}_{0}>0$, then there exists a unique sequence $\left\{x_{n}(0)\right\}_{0}^{\infty}$ satisfying $(1.5 .17)$; its gf $R_{0}$ has a positive radius of convergence, while for $|z|$ sufficiently small

$(1.5 .18) \quad R_{0}(z)=\frac{1}{z}\{1-P(0) / P(z)\}$.

If, in addition, all $r_{n}(0)$ 's are nonnegative, then necessarily

$(1.5 .19) \sum_{n=0}^{\infty} x_{n}(0)<1$.

(ii) If $\left\{r_{n}(0)\right\}_{0}^{\infty}$ is a sequence of nonnegative numbers satisfying (1.5.19), then there exists a unique lattice distribution $\left\{\mathrm{p}_{n}\right\}_{0}^{\infty}$ with $\mathrm{p}_{0}>0$, satisfying (1.5.17).

PROOF。

(i) The proof of the first part is similar to that of lemma 1.5.6. If $r_{n}(0) \geq 0$ for all $n \in \mathbb{N}_{0}$, then we can write

$$
1-p_{0}=\sum_{n=0}^{\infty} p_{n+1}=\sum_{n=0}^{\infty} \sum_{k=0}^{n} p_{k} x_{n-k}(0)=\sum_{n=0}^{\infty} x_{n}(0),
$$

from which $(1,5.19)$ follows.

(ii) If $x_{n}(0) \geq 0$ for all $n$ and if (1.5.19) holds, then it is seen that the function $P$, defined by $(1.5 .14)$ with $P(0)$ given by $(1.5 .15)$, is abs mon with $P(1)=1$. It follows that $P$ is the pgf of a lattice distribution $\left\{p_{n}\right\}_{0}^{\infty}$ that satisfies (1.5.17). The uniqueness of $\left\{p_{n}\right\}$ is evident from $(1.5 .17)$.

To conclude this section we mention two more classes of inf div lattice distributions: the classes of comp mon and log-convex lattice distributions. Comp mon has been introduced in definition 1.3.10; from theorem 1.3.11 one easily deduces the following lemma.

LEMMA 1.5.11. A lattice distribution $\left\{p_{n}\right\}_{0}^{\infty}$ is comp mon iff $\left\{p_{n}\right\}$ is a mixture of geometric distributions, i.e. iff there exists a df $G$ on $[0,1)$ such that 
$(1.5 .20) \quad p_{n}=\int_{[0,1)}(1-p) p^{n} d G(p) \quad\left(n \in \mathbb{N}_{0}\right)$.

Log-convexity can be introduced as follows.

DEFINITION 1.5.12. A lattice distribution $\left\{\mathrm{p}_{\mathrm{n}}\right\}_{0}^{\infty}$ is said to be $\log$-convex if $(1.5 .21) \quad p_{n}^{2} \leq p_{n-1} p_{n+1} \quad(n \in \mathbb{N}) \quad$.

Let us define the following four classes of lattice distributions $\left\{p_{n}\right\}_{0}^{\infty}$ with $p_{0}>0$ :

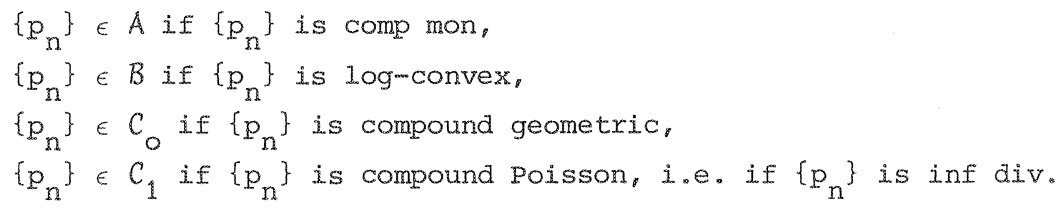

Then the family $(A, B, C, C$, defines a classification (cf. the end of section 2) of $C_{1}$, as will be apparent from the following relations (cf. Kaluza (1928), Goldie (1967), Steutel (1970) and Warde \& Katti (1971)).

THEOREM 1.5.13. $A \subset B \subset C_{0} \subset C_{1}$, where all inclusions are strict.

REMARK 1.5.14. "C $C_{0} \subset C_{1}$ " also easily follows from theorems 1.5 .3 and 1.5 .8 by use of the following relation between $R_{0}$ and $R_{1}$ :

$(1.5 .22) \quad R_{1}(z)=\left\{1-z R_{0}(z)\right\}^{-1} \frac{d}{d z}\left[z R_{0}(z)\right]$.

1.6. Infinitely divisible distributions on $[0, \infty)$

The inf div distributions on $[0, \infty)$ can be characterized in the following way (cf. Fellex (1971), ch. XIII).

THEOREM 1.6.1. A positive function $\varphi$ on $[0, \infty)$ is the PLST $\hat{F}$ of an inf div df $F$ on $[0, \infty)$ iff $\varphi(0)=1$ and the function $\varphi_{0}$, defined by

$(1.6 .1) \quad \varphi_{0}(\tau):=-\cdots \frac{d}{d \tau} \log \varphi(\tau) \quad(\tau>0)$,

is comp mon, or, equivalently, iff there exists a right-continuous, nondecreasing function $\mathrm{K}_{\mathrm{o}}$ on $\mathbb{R}$, satisfying 
$(1.6 .2) \int_{(1, \infty)} \frac{1}{x} d K_{0}(x)<\infty$,

such that $\varphi$ has the form

$(1.6 .3) \varphi(\tau)=\exp \left[\int_{[0, \infty)}\left(e^{-\tau x}-1\right) \frac{1}{x} d K_{0}(x)\right] \quad(\tau \geq 0)$.

We can (and will) choose the function $k_{0}$ such that $k_{0}$ vanishes on $(-\infty, 0)$. As we then also have $\hat{\mathrm{K}}_{0}=\varphi_{0}$, with $\varphi_{0}$ given by (1.6.1), the function $\mathrm{K}_{0}$ is uniquely determined by $\varphi=\hat{F} ; K_{0}$ is called the canonical function of the inf div df $F$. Its relation with the Levy canonical representation $\left(a, \sigma^{2}, M\right)$ will be shown in the next section.

Beforegiving some properties of $\mathrm{K}_{0}$, we state a characterization of the inf div df's on $[0, \infty)$ in terms of the df's themselves, which has been used by Steutel (1970), and can be obtained by inverting the expression for $\varphi^{\prime \prime}=\hat{F}$ ' in $(1.6 .3)$.

THEOREM 1.6.2. A df $\mathrm{F}$ on $[0, \infty)$ is inf div iff there exists a right-continuous, nondecreasing function $k_{0}$ such that

(1.6.4) $\int_{[0, x]} y d F(y)=\int_{[0, x]} F(x-y) d K_{0}(y) \quad(x \geq 0)$.

COROLIARY 1.6.3. A pdf $f$ on $(0, \infty)$ is inf div iff there exists a right-continuous, nondecreasing function $k_{0}$ such that

(1.6.5) $x f(x)=\int_{[0, x]} f(x-y) d x_{0}(y) \quad$ (almost all $\left.x>0\right)$.

Now we can prove the following properties of the canonical function $\mathrm{K}_{0}$.

LEMMA 1.6.4. Let $F$ be an inf div df with $\ell(F) \geq 0$ and canonical function $K_{0}$. Then

(i) $\mathrm{K}_{\mathrm{O}}(\mathrm{O})=\ell(\mathrm{F})$;

(ii) $\int_{(0, \infty)} \frac{1}{x} d K_{0}(x)<\infty$ iff $F(l(F))>0$, in which case the following relation holds:

$(1.6 .6) \quad \int_{(0, \infty)} \frac{1}{x} d K_{0}(x)=-\log F(l(F))$; 
(iii) $K_{0}$ is bounded iff $\mu_{1}:=\int_{(0, \infty)} x d F(x)<\infty$, in which case

$(1.6 .7) \quad \int_{[0, \infty)} d K_{0}(x)=\mu_{1}$

PROOF. Define the df $G$ by $G(x):=F(x+l(F))(x \in \mathbb{R})$, then $G$ is again inf div, with $\ell(G)=0$ and canonical function $L_{O}$, say. According to theorem 1.6.2, we have for all $x>0$

$$
G(x) L_{0}(0) \leq \int_{[0, x]} G(x-y) d L_{0}(y)=\int_{[0, x]} y d G(y) \leq x G(x),
$$

and hence, as $\ell(G)=0, L_{0}(0)=0$. Using the representation (1.6.3) for $\hat{G}_{\text {, }}$ we can write

$$
\hat{F}(\tau)=e^{-\ell(F) \tau} \tilde{G}(\tau)=\exp \left[-\ell(F) \tau+\int_{(0, \infty)}\left(e^{-\tau x}-1\right) \frac{1}{x} d L_{0}(x)\right] \quad(\tau \geq 0) .
$$

But as $\hat{F}$ can also be represented by $(1.6 .3)$, the uniqueness of the canonical function implies $k_{0}(0)=l(F)$. In view of $(1.6 .3)$ we can now write

$$
\log \left\{\hat{F}(\tau) e^{\ell(F) \tau}\right\}=\int_{(0, \infty)}\left(e^{-\tau x}-1\right) \frac{1}{x} d K_{0}(x) \quad(\tau \geq 0)
$$

from which, letting $\tau \rightarrow \infty$ and using $(1.3 .4)$ and the dominated convergence theorem, we obtain part (ii) of the lemma. Finally, using (1.3.6) and the fact that $\hat{\mathrm{K}}_{0}=\varphi_{0}$ with $\varphi_{0}$ given by $(1.6 .1)$, we obtain part (iii) as follows:

$$
\int \mathrm{dK}_{0}(x)=\lim _{\tau \downarrow 0} \hat{\mathrm{K}}_{0}(\tau)=\lim _{\tau \nmid 0}-\hat{\mathrm{F}}^{\prime}(\tau) \hat{\mathrm{F}}(\tau)=\mu_{1}(\leq \infty) .
$$

Part (iii) of the preceding lemma can be generalized to obtain necessary and sufficient conditions for the existence of higher moments of inf div distributions on $[0, \infty)$. This has already been done by wolfe (1971b) for ge-neral inf div distributions, but in our case the proof is very simple and we obtain a relation with the class $C_{1}$ of inf div lattice distributions.

THEOREM 1.6.5. Let $F$ be an inf div df on $[0, \infty)$ with canonical function $K_{0}$. Then for all $\mathrm{n} \in \mathbb{N}_{\mathrm{O}}$

(1.6.8) $\mu_{n+1}:=\int_{[0, \infty)} x^{n+1} d r(x)<\infty \Leftrightarrow \nu_{n}:=\int_{[0, \infty)} x^{n} d k_{0}(x)<\infty$, 
in which case

$$
\text { (1.6.9) } \quad \mu_{n+1}=\sum_{k=0}^{n}\left(\begin{array}{l}
n \\
k
\end{array}\right) \mu_{k} \nu_{n-k} ;
$$

if $\mu_{n}<\infty$ for all $n \in \mathbb{N}$, then $\left\{\mu_{n} / n !\right\}_{0}^{\infty}$ satisfies the recurrence relations (1.5.9) for $C_{1}$, with $r_{n}(1)=\nu_{n} / n$ : $\left(n \in \mathbb{N}_{0}\right)$.

PROOF。 If $U$ is a nonnegative, right-continuous, nondecreasing function with $\ell(U) \geq 0$, then obviously for all $\mathrm{n} \in \mathbb{N}_{\mathrm{O}}$

$$
\text { (1.6.10) } \lim _{\tau \downarrow 0}(-1)^{n}\left(\frac{d}{d \tau}\right)^{n} \bar{U}(\tau)=\int_{[0, \infty)} x^{n} d u(x) \quad(\leq \infty) .
$$

Since the canonical function $K_{0}$ of an inf div df $F$ on $[0, \infty)$ satisfies $-\hat{F}^{\prime}(\tau)=\hat{F}(\tau) \hat{K}_{0}(\tau)$ (cf. theorem 1.6 .1 or $(1.6 .4)$ ), we can write

$$
(1.6 .11) \quad(-1)^{n+1}\left(\frac{d}{d \tau}\right)^{n+1} \hat{F}(\tau)=(-1)^{n} \sum_{k=0}^{n}\left(\frac{n}{k}\right)\left\{\left(\frac{d}{d \tau}\right)^{k_{\hat{F}}(\tau)}\right\}\left\{\left(\frac{d}{d \tau}\right)^{n-k_{\hat{K}}}(\tau)\right\} .
$$

Now, using the fact that $\mu_{k}<\infty(k=0,1, \ldots, n)$ if $\mu_{n+1}<\infty$ and the same property of $\left\{\nu_{n}\right\}^{\infty}$, and letting $\tau \downarrow 0$ in $(1.6 .11)$ (cf. $\left.(1.6 .10)\right)$, we see the assertions of the theorem to be true.

If $F$ is an inf div df on $[0, \infty)$ with $F(0)>0$, then the representation $(1.6 .3)$ for $\hat{\mathrm{F}}$ can be simplified as follows.

THEOREM 1.6.6. A df $F$ on $[0, \infty)$ is inf div with $F(0)>0$ iff $F$ is compound Poisson, $1 . e$. iff $\hat{\mathrm{F}}$ has the form

$(1.6 .12) \quad \hat{F}(\tau)=\exp [\mu(\hat{G}(\tau)-1)] \quad(\tau \geq 0)$, where $\mu>0$ and $G$ is a df with $G(0)=0$.

PROOF. Let $F$ be an inf div df on $[0, \infty)$ with $F(0)>0$. Then $l(F)=0$, and if $\mathrm{K}_{0}$ is the canonical function of $\mathrm{F}$, it follows by lemma 1.6.4(ii) that

$$
\mu:=\int_{(0, \infty)} \frac{1}{x} d K_{0}(x)<\infty .
$$

Now if we define

$$
G(x):=\mu^{-1} \int_{(0, x]} \frac{1}{y} d k_{0}(y) \quad(x \geq 0) .
$$


then $G$ is a df with $G(0)=0$, and it is easily seen that the representation (1.6.3) for $\hat{\mathrm{F}}$ can be rewritten in the form (1.6.12).

Conversely, it is well known (and trivial) that a PLST of the form (1.6.12) is inf div with $F(0)>0$.

The compound geometric distributions on $[0, \infty)$, which are also compound Pois-son (cf. lemma 1.4.14), can be characterized by a functional equation similar to (1.6.4) (cf. Steutel (1970)). We use a notation that will be clarified in chapter 5 .

THEOREM 1.6.7. A df $\mathrm{F}$ on $[0, \infty)$ is compound geometric, i.e. has a PLST $\hat{\mathrm{F}}$ of the form

$(1.6 .13) \hat{F}(\tau)=\frac{1-p}{1-p \hat{G}(\tau)} \quad(\tau \geq 0)$,

where $0<p<1$ and $G$ is a df with $G(0)=0$, iff $F(0)>0$ and there exists a. right-continuous, nondecreasing function $K_{\infty}$ such that

$(1.6 .14) \quad F(x)-F(0)=\int_{[0, x]} F(x-y) d K_{\infty}(y) \quad(x \geq 0)$.

From (1.6.14) one easily proves the following result about moments of compound geometric df's on $[0, \infty$ ) (cf. (the proof of) theorem 1.6.5).

THEOREM 1.6.8. Let $F$ be a compound geometric $d f$ on $[0, \infty)$, and let $\mathrm{K}_{\infty}$ be the function in theorem 1.6.7. Then for all $n \in \mathbb{N}$

$(1.6 .15) \quad \mu_{n}:=\int_{[0, \infty)} x^{n} d F(x)<\infty \Leftrightarrow \nu_{n}:=\int_{[0, \infty)} x^{n} d_{\infty}(x)<\infty$,

in which case

$(1.6 .16) \quad \mu_{n}=\sum_{k=0}^{n}\left(\begin{array}{l}n \\ k\end{array}\right) \mu_{k} \nu_{n-k} ;$

if $\mu_{\mathrm{n}}<\infty$ for all $\mathrm{n} \in \mathbb{N}$, then $\left\{\mu_{\mathrm{n}} / \mathrm{n}:\right\}_{0}^{\infty}$ satisfies the recurrence relations $(1.5 .17)$ for $C_{0}$, with $x_{n}(0)=F(0)^{-1} \nu_{n+1} /(n+1):\left(n \in \mathbb{N}_{0}\right)$.

Finally, as in the discrete case (cf. section 5), we consider the following classes:

$D$ : the class of $\mathrm{df}^{\prime} \mathrm{s}$ on $[0, \infty)$ with a comp mon density, 
$E$ : the class of $\mathrm{df}^{\prime} \mathrm{s}$ on $[0, \infty)$ with a log-convex density.

Here log-convexity is defined as follows.

DEFINITION 1.6.9. A positive pdf $f$ on $(0, \infty)$ is said to be $20 g^{-m}$ convex if $\log f$ is convex, i.e. if

$(1.6 .17) \quad f(\lambda x+(1-\lambda) y) \leq f(x)^{\lambda} f(y)^{1-\lambda} \quad(x>0, y>0,0<\lambda<1)$.

In view of Bernstein's theorem (theorem 1.3.7) one easily verifies that the following characterization of the class $D$ holds.

LEMMA 1.6.10. A paf $f$ on $(0, \infty)$ is comp mon iff $f$ is a mixture of exponential distributions, i.e. iff there exists a df $G$ on $(0, \infty)$ such that

$(1.6 .18) \quad E(x)=\int_{(0, \infty)} \mu e^{-\mu x} \mathrm{dG}(\mu) \quad(x>0)$

The df's in the classes $D$ and $E$ are inf div; this has been proved by Goldie (1967) and by steutel (1970), respectively. In fact, denoting the class of all inf div df's on $[0, \infty)$ by $F_{0}$, we have the following partial analogue of theorem 1.5 .13$.

THEOREM 1.6.11. $D \subset E \subset F_{0}$.

In chapter 5 we shall introduce an analogue of the class $C_{0}$ for distributions on $[0, \infty)$; this class, called $F_{\infty}$, will fill the gap between $E$ and $F_{0}$ i.e. it will have the property that

$$
E \subset F_{\infty} \subset F_{0} \text {. }
$$

It follows that the family $\left(D, E, F_{\infty} F_{0}\right)$ defines a classification of $F_{0}$ which can be considered as an analogue of the classification of $C_{1}$, defined by $\left(A, B, C_{0}, C_{1}\right)$ (cf. section 5$)$, for distributions on $[0, \infty)$.

\subsection{Properties of and relations between canonical representations}

In theorem 1.4.7 the chf $\widetilde{F}$ of an inf div df $F$ on IR has been characterized by the Lêvy canonical representation $\left(a, \sigma^{2}, \mathrm{M}\right)$ :

$$
\text { (1.7.1a) } \tilde{F}(t)=\exp \left[i t a-\frac{1}{2} \sigma^{2} t^{2}+\int_{\mathbb{I R} \backslash\{0\}}\left\{e^{i t x}-1-\frac{i t x}{1+x^{2}}\right\} d M(x)\right] \quad(t \in \mathbb{R})
$$


where $a \in \mathbb{R}, \sigma^{2} \geq 0$ and $M$ is a right-continuous function on $\mathbb{R} \backslash\{0\}$ with the following properties: $M$ is nondecreasing on $(-\infty, 0)$ and on $(0, \infty), M(-\infty)=$ $=M(\infty)=0$ and

$(1.7 .1 b)$

$$
\int_{(-1,1) \backslash\{0\}} x^{2} d M(x)<\infty .
$$

The chf $\tilde{F}$ of an inf div df $F$ on $[0, \infty)$ can be represented by means of its canonical function $\mathrm{K}_{0}$ ( $\mathrm{Cf}$. theorem 1.6.1):

$$
(1.7 .2 a) \quad \tilde{F}(t)=\exp \left[\int_{[0, \infty)}\left\{e^{i t x}-1\right\} \frac{1}{x} d K_{0}(x)\right] \quad(t \in \mathbb{R}),
$$

where $\mathrm{K}_{0}$ is a right--continuous, nondecreasing function, vanishing on $(-\infty, 0)$ and satisfying

$$
(1.7 .2 b) \quad \int_{(1, \infty)} \frac{1}{x} d \mathrm{~K}_{0}(\mathrm{x})<\infty
$$

Finally, the chf $\tilde{F}$ of an inf div df $F$ on $\mathbb{N}_{0}$ with $F(0)>0$ has the following form (cf. theorem 1.5.3):

$$
\text { (1.7.3a) } \tilde{F}(t)=\exp \left[\sum_{n=0}^{\infty}\left\{e^{i t(n+1)}-1\right\} \frac{1}{n+1} x_{n}(1)\right] \quad(t \in \mathbb{I R})
$$

where $\left\{r_{n}(1)\right\}_{0}^{\infty}$ is a sequence of nonnegative numbers, satisfying

$$
\text { (1.7.3b) } \sum_{n=0}^{\infty} \frac{r_{n}(1)}{n+1}<\infty \text {. }
$$

In fact, the three classes of $d f^{\prime \prime}$, considered in $(1.7 .1 a),(1.7 .2 a)$ and $(1.7 .3 a)$, respectively, define a classification of the class of all inf div df's on IR. NOw we want to investigate under what conditions on the canonical. quantities an inf div df belongs to one of the subclasses and what relations exist between the canonical representations. Not all of this is new, but it seems useful to collect the available information, together with a few additions, and, sometimes, simpler proofs.

First we modify $(1.7 .2 a)$ in such a way that we get a representation for all inf div df"s $F$ with $\ell(F)>-\infty$.

THEOREM 1.7.1. A function $\varphi$ on $\mathbb{R}$ is the chf $\tilde{F}$ of an inf div df $F$ with $\ell(F)>\ldots \infty$ iff $\varphi$ has the form 
$(1.7 .4 a) \quad \varphi(t)=\exp \left[i t \gamma+\int_{(0, \infty)}\left\{e^{i t x}-1\right\} \mathrm{dN}(x)\right] \quad(t \in \mathbb{R})$,

where $\gamma \in \mathbb{R}$ and $N$ is a right-continuous, nondecreasing function on $(0, \infty)$ with $N(\infty)=0$ and satisfying

$(1.7 .4 \mathrm{~b}) \quad \int_{(0,1]} \mathrm{xdN}(\mathrm{x})<\infty$.

The representation $(\gamma, N)$ is unique, and necessarily $l(F)=\gamma_{0}$

PROOF. Let $F$ be an inf div df with $\ell(F)>-\infty$. The df $F_{1}$, defined by $F_{1}(x):=F(x+\ell(F)) \quad(x \in \mathbb{R})$, is inf div with $\ell\left(F_{1}\right)=0$, and hence $\tilde{F}_{1}$ has the form $(1.7 .2 a)$ with $K_{0}(0)=\ell\left(F_{1}\right)=0$ (cf. lemma 1.6.4(i)). It follows that

$$
\tilde{F}(t)=\exp \left[i t l(F)+\int_{(0, \infty)}\left\{e^{i t x}-1\right\} \frac{1}{x} d K_{0}(x)\right] \quad(t \in \mathbb{R}) .
$$

Because of $(1,7.2 \mathrm{~b})$ we can define a function $N$ on $(0, \infty)$ by

$$
N(x):=-\int_{(x, \infty)} \frac{1}{y} d K_{0}(y) \quad(x>0) .
$$

Then $\mathbb{N}$ is right-continuous and nondecreasing, and satisfies $(1.7 .4 b)$ :

$$
\int_{(0,1]} x \mathrm{dN}(\mathrm{x})=\int_{[0,1]} d \mathrm{~K}_{0}(\mathrm{x})=\mathrm{K}_{0}(1)<\infty .
$$

while $\widetilde{F}$ takes the form $(1.7 .4 a)$ with $\gamma=\ell(F)$. The representation $(\gamma, N)$ is unique as $\mathrm{K}_{0}$ is unique.

Conversely, if a function $\varphi$ has the form $(1.7 .4 a)$, then $\varphi_{1}(t):=e^{-i t \gamma} \varphi(t)$ has the form $(1.7 .2 a)$ with $K_{0}$ defined by (cf. $(1.7 .4 b)$ )

$$
K_{0}(x):=\int_{(0, x]} y d N(y) \quad(x>0) .
$$

By theorem 1.6.1 and lemma 1.6.4(i) it follows that $\varphi_{1}$ is the che of an inf div df $F_{1}$ with $\ell\left(F_{1}\right)=0$, and hence $\varphi(t)=e^{i t \gamma} \varphi_{1}(t)$ is the chf of an inf div df $F$ with $\ell(F)=\gamma$.

COROLIARY 1.7.2. If $F$ is an inf div df with $\ell(F) \geq 0$, then the following relation holds between its canonical function $k_{0}$ and its representation 
$(\gamma, \mathbb{N})$ from $(1.7 .4 a):$

$(1.7 .5) \quad K_{0}(x)=\gamma+\int_{(0, x]} \operatorname{ydN}(y) \quad(x \geq 0)$.

Using theorem 1.7.1 we can give necessary and sufficient conditions for an inf div df $F$ to have a support that is bounded from below, i.e. $\ell(F)>-\infty$. This has also been done by Baxter \& Shapiro (1960) (see also Fellex (1971), Ch. XVII), but our method of making use of the representation (1.7.2a) for inf div df's on $[0, \infty)$, instead of using only $(1.7 .1 a)$, simplifies matters. Also, the expression for $l(F)$ to be given in $(1.7 .7)$ follows much more directly than in Tucker (1961).

THEOREM 1.7.3. Let $\mathrm{F}$ be an inf div df with Lëvy canonical representation $\left(a, \sigma^{2}, M\right)$. Then $\ell(F)>-\infty$ iff $\sigma^{2}=0, M \equiv 0$ on $(-\infty, 0)$ and

$(1.7 .6) \quad \int_{(0,1]} \operatorname{xdM}(x)<\infty$.

in which case necessarily

$(1.7 .7) \quad \ell(F)=a-\int_{(0, \infty)} \frac{x}{1+x^{2}} d M(x)$.

PROOF. Let $\ell(F)>-\infty$. Then by theorem $1.7 .1 \tilde{F}$ has the representation $(\gamma, N)$ from $(1.7 .4 \mathrm{a})$ with, because of $(1.7 .4 \mathrm{~b})$,

$$
\int_{(0, \infty)} \frac{x}{1+x^{2}} d N(x)<\infty
$$

It follows that $\tilde{\mathrm{F}}$ can be written in the form

$$
\tilde{F}(t)=\exp \left[i t\left\{\gamma+\int_{(0, \infty)} \frac{x}{1+x^{2}} d N(x)\right\}+\int_{(0, \infty)}\left\{e^{i t x}-1-\frac{i t x}{1+x^{2}}\right\} d N(x)\right],
$$

from which by the uniqueness of the representation $\left(a, \sigma^{2}, M\right)$ it is seen that $\sigma^{2}=0, M \equiv 0$ on $(-\infty, 0)$ and

$$
\text { (1.7.8) } M \equiv N \text { on }(0, \infty), a=\gamma+\int_{(0, \infty)} \frac{x}{1+x^{2}} d N(x) \text {. }
$$

Conversely, if $\left(a, \sigma^{2}, M\right)$ satisfies the conditions of the theorem, then the 
integral in (1.7.7) is finite. Hence the representation (1.7.1a) for $\tilde{F}$ can be written in the form $(1.7 .4 a)$ with $\gamma$ given by the right-hand side of $(1.7 .7)$ and $N \equiv M$ on $(0, \infty)$. By theorem 1.7 .1 it now follows that $\ell(F)>-\infty$, and as $\ell(F)=\gamma$, we have $(1.7 .7)$.

COROLLARY 1.7.4. If $F$ is an inf $\operatorname{div}$ df with $\ell(F) \geq 0$, then the following relations hold between its canonical function $K_{O}$ and the Lëvy representation $\left(a, \sigma^{2}, M\right)$ :

(1.7.9) $K_{0}(x)=\left\{a-\int_{(0, \infty)} \frac{x}{1+x^{2}} d M(x)\right\}+\int_{(0, x]} y d M(y) \quad(x \geq 0)$.

and, conversely,

$(1.7 .10) \quad a=\int_{[0, \infty)} \frac{1}{1+x^{2}} d K_{0}(x), M(x)=-\int_{(x, \infty)} \frac{1}{y} d K_{0}(y) \quad(x>0)$.

PROOF. Use the relations $(1.7 .5)$ and $(1.7 .8)$

Using the same technique as in the proofs of lemma 1.6.4(ii) and theorem 1.6.6, we obtain a generalization of these results to all inf div df's $F$ with $\ell(F)>-\infty$.

THEOREM 1.7.5. Let $F$ be an inf div df with Lévy representation $\left(a, \sigma^{2}, M\right)$ and with $\ell(F)>-\infty$. Then $F(\ell(F))>0$ iff $M$ is bounded, in which case

$(1.7 .11)-\log F(\ell(F))=M(0+)$,

and $F$ is a shifted compound poisson df on $[0, \infty)$, i.e. $\tilde{F}$ has the form

$(1.7 .12) \quad \tilde{F}(t)=e^{i . t \ell(F)} \exp [\mu(\tilde{G}(t)-1)] \quad(t \in \mathbb{I R})$,

where $\mu>0$ and $G$ is a df with $G(0)=0$.

COROLIARY 1.7.6. If $F$ is an inf div df with $\ell(F)>-\infty$, then $F$ is continuous at $\ell(F)$ iff $F$ is continuous everywhere.

PROOF. If $F$ is continuous at $l(F)$, then by the preceding theorem $M$ is unbounded. Now, by a result of Blum \& Rosenblatt (1959) (our theorem 1.7.9) it follows that $F^{\prime}$ is continuous everywhere. 
We now consider the class of inf div lattice distributions $\left\{p_{n}\right\}_{0}^{\infty}$ with $p_{0}>0$ as a subclass of the class of inf div distributions on $[0, \infty)$. Comparing the canonical representations $(1.7 .2 \mathrm{a})$ and $(1.7 .3 \mathrm{a})$, and the conditions $(1.7 .2 \mathrm{~b})$ and $(1.7 .3 \mathrm{~b})$, we are immediately led to the following result.

THEOREM 1.7.7. Let $F$ be an inf div df on $[0, \infty)$ with canonical function $K_{0}$ Then $F$ is the df of a lattice distribution $\left\{p_{n}\right\}_{0}^{\infty}$ with $p_{0}>0$ iff $k_{0}$ is a step function with discontinuities restricted to $\mathbb{N}$; in this case the following relations exist between $K_{0}$ and the canonical sequence $\left\{x_{n}(1)\right\}_{0}^{\infty}$ of $\left\{p_{n}\right\}$ : $(1.7 .13) \quad K_{0}(x)=\sum_{n=1}^{\infty} x_{n-1}(1) 1_{[n, \infty)}(x) \quad(x \in \mathbb{R})$ and, conversely,

$(1.7 .14) \quad r_{n}(1)=K_{0}(n+1)-K_{0}(n) \quad\left(n \in \mathbb{N}_{0}\right)$.

COROLLARY 1.7.8. Let $\mathrm{F}$ be an inf div df with Lêvy representation $\left(a, \sigma^{2}, \mathrm{M}\right)$. Then $F$ is the df of a lattice distribution $\left\{p_{n}\right\}_{0}^{\infty}$ with $p_{0}>0$ iff $\sigma^{2}=0$, $M$ is a step function with discontinuities restricted to $\mathbb{N}$ and

$$
(1.7 .15) \quad a=\int_{(0, \infty)} \frac{x}{1+x^{2}} d M(x)
$$

in this case the following relations exist between $M$ and the canonical sequence $\left\{x_{n}(1)\right\}_{0}^{\infty}$ of $\left\{p_{n}\right\}$ :

$(1.7 .16) \quad M(x)=-\sum_{n=0}^{\infty} \frac{x_{n}(1)}{n+1} 1_{(0, n+1)}(x) \quad(x>0)$,

and, conversely,

$(1.7 .17) \quad r_{n}(1)=(n+1)\{M(n+1)-M(n)\} \quad\left(n \in \mathbb{N}_{0}\right)$.

The first part of this corollary is a special case of part (i) of the following result, due to Blum \& Rosenblatt (1959).

THEOREM 1.7.9. Let $F$ be an inf div de with Lévy representation $\left(a, \sigma^{2}, M\right)$. Then

(i) $F$ is discrete iff $\sigma^{2}=0, M$ is bounded and $M$ is a step function,

(ii) $F$ is continuous iff $\sigma^{2}>0$ or $M$ is unbounded. 
Also, for an inf div df to be absolutely continuous, necessary and sufficient conditions are known (cf. Tucker (1964)). We confine ourselves to the following simple sufficient conditions (cf. Tucker (1962) and Fisz \& Varadarajan (1963)), and return to this in chapter 5.

THEOREM 1.7.10. Let $F$ be an inf div df with Lêvy representation $\left(a, \sigma^{2}, M\right)$. If $\sigma^{2}>0$ or if there exists $\varepsilon>0$ such that $M$ is unbounded and absolutely continuous on $(-\varepsilon, 0)$ or on $(0, \varepsilon)$, then $F$ is absolutely continuous.

Finally, we consider two more classes of inf div df's for which the representation (1.7.1a) has a simplex form. Let $F$ be an inf div df without a normal component, i.e. with $\sigma^{2}=0$ in its Lévy representation $\left(a, \sigma^{2}, M\right)$. The function $M$ satisfies (cf. (1.7.1b))

$(1.7 .18) \quad \int_{(-1,1) \backslash\{0\}} x^{2} \mathrm{dM}(\mathrm{x})<\infty$.

and now we want to consider the special cases where

(1.7.19) $\int_{(-1,1) \backslash\{0\}}|\mathrm{x}| \mathrm{dM}(\mathrm{x})<\infty$.

or

$(1.7 .20) \quad \int_{(-1,1) \backslash\{0\}} d M(x)<\infty$ (i.e. $M$ is bounded) .

If $M \equiv 0$ on $(-\infty, 0)$, then, according to theorems 1.7 .3 and $1.7 .5,(1.7 .19)$ and $(1.7 .20)$ give rise to the inf div df's $F$ with $\ell(F)>-\infty$ and to the shifted compound poisson distributions on $[0, \infty)$, respectively. In the general case we have analogous results, the first of which we shall need in the last section of chapter 5 .

THEOREM 1.7.11. Let $F$ be an inf div df with Lêvy representation $\left(a, \sigma^{2}, M\right)$. Then there exist inf $\operatorname{div} d f^{i} \mathrm{~s}_{1}$ and $\mathrm{F}_{2}$ on $[0, \infty)$ such that

$(1.7 .21) \quad \tilde{F}(t)=\tilde{F}_{1}(t) \tilde{F}_{2}(-t) \quad(t \in \mathbb{R})$

iff $\sigma^{2}=0$ and $(1.7 .19)$ holds.

PROOF. Let $F^{\prime}$ satisfy $(1.7 .21)$ with $F_{1}$ and $F_{2}$ inf div $d f^{i} s$ on $[0, \infty)$. Then by theorem 1.7 .1 for $j=1,2 \tilde{F}_{j}$ has the representation $\left(\gamma_{j}, N_{j}\right)$ given by 
$(1.7 .4 a)$, and hence

$(1.7 .22) \quad \tilde{F}(t)=\exp \left[i t\left(\gamma_{1}-\gamma_{2}\right)+\int_{(0, \infty)}\left(e^{i t x}-1\right) d N_{1}(x)+\int_{(0, \infty)}\left(e^{-i t x}-1\right) d N_{2}(x)\right]$.

Now by $(1.7 .1 a)$ it follows that the Levvy representation $\left(a, \sigma^{2}, M\right)$ of $F$ satisfies

$(1.7 .23) M(x)=\left\{\begin{array}{ll}N_{1}(x), & \text { if } x>0 \\ -N_{2}(-x-), & \text { if } x<0\end{array}, \sigma^{2}=0, a=\gamma_{1}-\gamma_{2}+\int_{\mathbb{R} \backslash\{0\}} \frac{x}{1+x^{2}} d M(x) ，\right.$

so that $\sigma^{2}=0$ and $M$ satisfies $(1.7 .19)$, because of $(1.7 .4 \mathrm{~b})$.

The converse can be shown in a similar way; we choose the $d f^{\prime} S F_{1}$ and $F_{2}$ in (1.7.21) by giving their representations $\left(\gamma_{1}, N_{1}\right)$ and $\left(\gamma_{2}, N_{2}\right)$, where we note that $N_{1}$ and $N_{2}$ are completely determined by $M$, but that the nonnegative quantities $\gamma_{1}$ and $\gamma_{2}$ only need to satisfy the equality in (1.7.23).

COROLIARY 1.7.12. Let $\mathrm{F}$ be an inf div df with Lévy representation $\left(a, \sigma^{2}, M\right)$. Then there exists an inf div df $\mathrm{F}_{1}$ on $[0, \infty)$ such that

$$
\begin{gathered}
(1.7 .24) \quad \widetilde{F}(t)=\widetilde{F}_{1}(t) \widetilde{F}_{1}(-t) \quad(t \in \mathbb{R}) \\
\text { iff } a=\sigma^{2}=0, M(x)=-M(-x-) \quad(x>0) \text { and } \\
\int_{(0,1]} x d M(x)<\infty,
\end{gathered}
$$

in which case $\tilde{F}$ can be given the following form:

$$
(1.7 .25) \tilde{F}(t)=\exp \left[2 \int_{(0, \infty)}(\cos t x-1) d M(x)\right] \quad(t \in \mathbb{R}) .
$$

THEOREM 1.7.13. Let $F$ be an inf div df with Lévy representation $\left(a, \sigma^{2}, M\right)$. Then $F$ is a shifted compound Poisson df, $i . e . \tilde{F}$ has the form

$(1.7 .26) \quad \widetilde{F}(t)=e^{i t \gamma} \exp [\mu(\tilde{G}(t)-1)] \quad(t \in \mathbb{R})$,

where $\gamma \in \mathbb{R}, \mu>0$ and $G$ is a df continuous at zero, iff $\sigma^{2}=0$ and $(1.7 .20)$ holds. The representation $(\gamma, \mu, G)$ in $(1.7 .26)$ is unique.

PROOF. If $\sigma^{2}=0$ and $(1.7 .20)$ holds, then it is easily verified that (1.7.1a) takes the form $(1.7 .26)$ by putting 
(1.7.27) $\gamma:=a-\int_{\mathbb{R} \backslash\{0\}} \frac{x}{1+x^{2}} d M(x), \mu:=\int_{\mathbb{R} \backslash\{0\}} d M(x), G(x):=\frac{1}{\mu} M(x)+1[0, \infty)(x)$

Conversely, if $F$ satisfies $(1.7 .26)$, then, comparing this equation with $(1.7 .1 a)$, we see that

(1.7.28) $\quad a=\gamma+\mu \int_{(-\infty, \infty)} \frac{x}{1+x^{2}} d G(x), \sigma^{2}=0, M(x)=\mu\left\{G(x)-1_{[0, \infty)}(x)\right\}$, so that $\sigma^{2}=0$ and $M$ is bounded.

Finally, suppose that $\tilde{\mathrm{F}}$ can be represented by $(1.7 .26)$ in two ways: by $\left(\gamma_{1}, \mu_{1}, G_{1}\right)$ and $\left(\gamma_{2}, \mu_{2}, G_{2}\right)$, say. Then necessarily

$$
i t \gamma_{1}+\mu_{1}\left(\tilde{G}_{1}(t)-1\right)=i t \gamma_{2}+\mu_{2}\left(\tilde{G}_{2}(t)-1\right)+2 k \pi i \quad(t \in \mathbb{R} ; k \in q) .
$$

Taking $t=0$ and using the continuity of $\widetilde{G}_{1}$ and $\widetilde{G}_{2}$, we get

$(1.7 .29) \quad i t\left(\gamma_{1}-\gamma_{2}\right)=\mu_{2}\left(\tilde{G}_{2}(t)-1\right)-\mu_{1}\left(\tilde{G}_{1}(t)-1\right) \quad(t \in \mathbb{I R})$.

It follows that

$$
\left|\gamma_{1}-\gamma_{2}\right||t| \leq 2\left(\mu_{1}+\mu_{2}\right) \quad(t \in \mathbb{R}),
$$

and hence $\gamma_{1}=\gamma_{2}$. Now, as the jump of a df $G$ at zero is given by (cf. Lukacs (1970), ch.3)

$(1.7 .30) G(0)-G(0-)=\lim _{T \rightarrow \infty} \frac{1}{2 T} \int_{(-T, T)} \tilde{G}(t) d t$

and as $G_{1}$ and $G_{2}$ are supposed to be continuous at zero, it follows from (1.7.29) with $\gamma_{1}=\gamma_{2}$ that $\mu_{1}=\mu_{2}$, and hence $G_{1}=G_{2}$.

Combining theorems 1.7 .9 (ii) and 1.7 .13 , we obtain the following result.

COROLLARY 1.7.14. An inf div df $F$ that is not continuous, has a chf $\tilde{F}$ of the form $(1.7 .26)$. 


\section{CHAPTER 2}

CLASSIFICATION OF THE INFINITELY DIVISIBLE LATTICE DISTRIBUTTONS

In section 1.5 we introduced four classes of inf div lattice distributions: the classes $C_{1}, C_{0}, B$ and $A$ of compound Poisson (i.e. inf div), compound geometric, logmconvex and comp mon distributions, respectively. Also, we noted that, as in this order these classes contain increasingly special dism tributions (cf. theorem 1.5 .13 ), they define a classification (cf. the end of section 1.2) of the inf div lattice distributions. The distributions in $A$ and $B$, and to some extent in $C_{0}$ are often easily recognized as such. Now, in order to get a better understanding of the class $C_{1} \backslash C_{0}$, we want to classify the distributions in this class.

To obtain such a classification, in section 1 we introduce classes of lattice distributions characterized by means of recurrence relations. These relations depend on cextain sequences $\left\{c_{n}^{*}(\alpha)\right\}$, and the main problem will be to choose $c_{n}^{*}(\alpha)$ in such a way that the resulting classes yield a classification of $C_{1}$ (section 2). This turns out to be possible by choosing a special sequence $\left\{c_{n}^{*}(\alpha)\right\}$; in section 3 the classes $c_{\alpha}(0 \leq \alpha \leq 1)$, resulting from this special choice, are shown to be increasing with $\alpha$. It turns out that an important role is played here by the compound geometric distributions. Pxoperties of the $C_{0}^{\prime}$ 's are proved in section 4 , where also some examples are given. In section 5 two other classifications of $C_{1}$ are discussed. Specifically, we considex the classes of compound negative-binomial distributions, which turn out to have properties similar to those of the $C_{\alpha}$ "s.

\subsection{Interpolating between $\mathrm{C}_{0}$ and $\mathrm{C}_{1}$}

The class $C_{1}$ of inf div (i.e. compound Poisson) lattice distributions $\left\{p_{n}\right\}^{\infty}$ with $0<p_{0}<1$, can be characterized as follows (cf. corollary 1.5.5): $\left\{\mathrm{p}_{\mathrm{n}}\right\} \in \mathrm{C}_{1}$ iff there exist nonnegative quantities $r_{n}(1)\left(\mathrm{n} \in \mathbb{N}_{0}\right.$ ) such that

$$
\text { (2.1.1) }(n+1) p_{n+1}=\sum_{k=0}^{n} p_{k} r_{n-k}(1) \quad\left(n \in \mathbb{N}_{0}\right) .
$$

Similarly, in corollary 1.5 .9 the class $C_{0}$ of compound geometric lattice distributions $\left\{\mathrm{p}_{\mathrm{n}}\right\}_{0}^{\infty}$ is characterized by the nonnegativity of quantities $x_{n}(0)$ satisfying

$$
(2.1 .2) \quad p_{n+1}=\sum_{k=0}^{n} p_{k} r_{n-k}(0) \quad\left(n \in \mathbb{N}_{0}\right)
$$


We know that $\mathcal{C}_{0} \subset C_{1}$ (cf. theorem 1.5.13), and the recurrence relations $(2.1 .1)$ and $(2.1 .2)$ now suggest the idea of generalizing them in order to obtain classes of lattice distributions that fill the gap between $C_{0}$ and $C_{1}$. Proceeding in such a way, for $0 \leq \alpha \leq 1$ we consider sequences $\left\{c_{n}^{*}(\alpha)\right\}_{0}^{\infty}$ with the following properties:

(2.1.3) $\left\{\begin{array}{l}c_{n}^{*}(0)=1, c_{n}^{*}(1)=n+1 \quad\left(n \in \mathbb{N}_{0}\right) \\ c_{n}^{*}(\alpha) \text { is nondecreasing in both } n \text { and } \alpha .\end{array}\right.$

For any choice of $\left\{\mathrm{c}_{\mathrm{n}}^{*}(\alpha)\right\}$ satisfying (2.1.3) we introduce classes $\mathrm{C}_{\alpha}^{*}$ $(0 \leq \alpha \leq 1)$ as follows.

DEFINITION 2.1.1. For $0 \leq \alpha \leq 1$ a lattice distribution $\left\{p_{n}\right\}_{0}^{\infty}$, with $p_{0}>0$, is said to be in the class $C_{\alpha}^{*}$ if there exist nonnegative quantities $x_{n}^{*}(\alpha)$ (n $\in \mathbb{N}_{0}$ ) such that

$(2.1 .4) \quad c_{n}^{*}(\alpha) p_{n+1}=\sum_{k=0}^{n} p_{k} x_{n-k}^{*}(\alpha) \quad\left(n \in \mathbb{N}_{0}\right)$

REMARK. The symbol * is added to the quantities $c_{n}^{*}(\alpha), r_{n}^{*}(\alpha)$ and $C_{\alpha}^{*}$ to enable us to use the notation without * for the special situation that will be considered from section 3 on. Further, as already noted in section 1.2, if $\left\{p_{n}\right\} \in C_{\alpha}^{*}$ with pgf $P$, then we shall also say that $P \in C_{\alpha}^{*}$.

clearly, taking $\alpha=0$ and $\alpha=1$ in definition 2.1 .1 , we get $C_{0}^{*}=C_{0}$ and $c_{1}^{*}=c_{1}$. For these cases the recurrence relations $(2.1 .4)$ have been considered in detail in section 1.5. Now, before choosing a special sequence $\left\{\mathrm{c}_{n}^{*}(\alpha)\right\}$, we discuss some general properties of (2.1.4) with an arbitrary $\alpha \in[0,1]$. We need the following notation (cf. $(2.1 .3)$ ):

$(2.1 .5) \quad C^{*}(\alpha):=\lim _{n \rightarrow \infty} C_{n}^{*}(\alpha) \quad(\leq \infty ; 0 \leq \alpha \leq 1)$, and for a given lattice distribution $\left\{p_{n}\right\}_{0}^{\infty}$

$(2.1 .6) \quad A_{\alpha}^{*}(z):=\sum_{n=0}^{\infty} C_{n}^{*}(\alpha) p_{n+1} z^{n} \quad(|z|<1 ; 0 \leq \alpha \leq 1)$. 
LEMMA 2.1.2. Consider a fixed sequence $\left\{c_{n}^{*}(\alpha)\right\}_{0}^{\infty}$ satisfying (2.1.3), and a fixed $\alpha \in[0,1]$.

(i) Let $\left\{\mathrm{p}_{\mathrm{n}}\right\}_{0}^{\infty}$ be a lattice distribution with $\mathrm{p}_{0}>0$. Then there exists a unique sequence $\left\{x_{n}^{*}(\alpha)\right\}_{0}^{\infty}$ satisfying (2.1.4), and its gf has a positive radius of convergence. If $r_{n}^{*}(\alpha) \geq 0$ for all $n \in \mathbb{N}_{0}$ (i.e. if $\left\{p_{n}\right\} \in C_{\alpha}^{*}$ ), then

(2.1.7) $\sum_{n=0}^{\infty} x_{n}^{*}(\alpha) / c_{n}^{*}(\alpha)<\infty$;

if furthermore $c^{*}(\alpha)$ is finite, then

(2.1.8) $\sum_{n=0}^{\infty} r_{n}^{*}(\alpha)<c^{*}(\alpha)$

(ii) Let $\left\{x_{n}^{*}(\alpha)\right\}_{0}^{\infty}$ be a sequence of nonnegative numbers satisfying (2.1.7), or, if $c^{*}(\alpha)$ is finite, $(2.1 .8)$. Then there exists a unique lattice distribution $\left[p_{n}\right]_{0}^{\infty}$ with $p_{0}>0$, satisfying $(2.1 .4)$.

PROOF。

(i) Let $\left\{\mathrm{p}_{n}\right\}_{\circ}^{\infty}$ be a lattice distribution with $\mathrm{p}_{0}>0$. Then, rewriting (2.1.4) in the form

$$
p_{0} x_{n}^{*}(\alpha)=c_{n}^{*}(\alpha) p_{n+1}-\sum_{k=1}^{n} p_{k} x_{n-k}^{*}(\alpha) \quad\left(n \in \mathbb{N}_{0}\right)
$$

we see that the sequence $\left\{x_{n}^{*}(\alpha)\right\}_{0}^{\infty}$, recursively defined by this equation, is the unique solution of $(2.1 .4)$. Further, as $p_{0}>0$, the function $R_{\alpha}^{*}$, defined by

$$
R_{\alpha}^{*}(z):=A_{\alpha}^{*}(z) / P(z)
$$

is analytic in $|z|<\varepsilon$ for some $\varepsilon>0$. It follows that the coefficients in the power-series expansion of $R_{\alpha}^{*}$ satisfy (2.1.4), and hence are equal to the $x_{n}^{*}(\alpha)^{*}$ s. So, for $|z|<\varepsilon$ the gf of $\left\{r_{n}^{*}(\alpha)\right\}$ coincides with $R_{\alpha}^{*}$ and therefore has a positive radius of convergence.

If all $r_{n}^{*}(\alpha)$ s s are nonnegative, then we can write

$$
\begin{aligned}
1-p_{0} & =\sum_{n=0}^{\infty} p_{n+1}=\sum_{n=0}^{\infty} c_{n}^{*}(\alpha)^{-1} \sum_{k=0}^{n} p_{k} x_{n-k}^{*}(\alpha)= \\
& =\sum_{k=0}^{\infty} p_{k} \sum_{n=0}^{\infty} r_{n}^{*}(\alpha) / c_{n+k}^{*}(\alpha) .
\end{aligned}
$$


From this it follows that

$$
\sum_{n=0}^{\infty} x_{n}^{*}(\alpha) / c_{n}^{*}(\alpha) \leq\left(1-p_{0}\right) / p_{0}<\infty
$$

while, in case $c^{*}(\alpha)$ is finite, by the monotonicity of $c_{n}^{*}(\alpha)$ we conclude that

$$
\sum_{n=0}^{\infty} r_{n}^{*}(\alpha) \leq\left(1-p_{0}\right) c^{*}(\alpha)<c^{*}(\alpha)
$$

(ii) Let $\left\{r_{n}^{*}(\alpha)\right\}_{0}^{\infty}$ be a sequence of nonnegative numbers satisfying (2.1.7). As $A^{*} \alpha^{\prime}$ defined by $(2.1 .6)$, is not expressible in terms of $P$, it is not possible to use the method applied in the cases $\alpha=0$ and $\alpha=1$; we have to work with the recurrence relations themselves. First consider the quantities $\gamma_{k}$, for $k \in \mathbb{N}_{0}$ defined by

$$
\gamma_{k}:=\sum_{n=0}^{\infty} r_{n}^{*}(\alpha) / c_{n+k}^{*}(\alpha) .
$$

Because of the monotonicity of $\mathrm{c}_{n}^{*}(\alpha)$ the $\gamma_{k}{ }^{*}$ s form a nonincreasing sequence of nonnegative numbers. Using the dominated convergence theorem one easily sees that $\lim _{k \rightarrow \infty} \gamma_{k}=0$, if $c^{*}(\alpha)$ is infinite, and that $\lim _{k \rightarrow \infty} \gamma_{k}<1$, if $c^{*}(\alpha)$ is finite and $(2.1 .8)$ holds. It follows that in both cases it is possible to choose $\gamma<1$ and $k \in \mathbb{N}$ such that $\gamma_{k} \leq \gamma$ for all $k>K$. Next we note that for any choice of $\mathrm{p}_{\mathrm{O}}>0(2.1 .4)$ has exactly one solution $\left\{\mathrm{p}_{\mathrm{n}}\right\}_{0}^{\infty}$ with, necessarily, $\mathrm{p}_{\mathrm{n}} \geq 0$ for all $\mathrm{n}$. Considering such a solution, for $\mathrm{N}>\mathrm{K}$ we can write

$$
\begin{aligned}
\sum_{n=1}^{N+1} p_{n} & =\sum_{n=0}^{N} c_{n}^{*}(\alpha)-1 \sum_{k=0}^{n} p_{k} x_{n-k}^{*}(\alpha)=\sum_{k=0}^{N} p_{k} \sum_{n=k}^{N} x_{n-k}^{*}(\alpha) / c_{n}^{*}(\alpha) \leq \\
& \leq \sum_{k=0}^{N} p_{k} \gamma_{k} \leq \sum_{k=0}^{K} p_{k} \gamma_{0}+\sum_{k=k+1}^{N} p_{k} \gamma \leq \gamma_{0} \sum_{k=0}^{K} p_{k}+\gamma \sum_{k=1}^{N+1} p_{k} .
\end{aligned}
$$

Hence, as $\gamma<1$,

$$
\sum_{n=1}^{N+1} p_{n} \leq \frac{\gamma_{0}}{1-\gamma} \sum_{k=0}^{K} p_{k} \quad(N>K),
$$

from which it follows that for all solutions $\left\{p_{n}\right\}_{0}^{\infty}$ of $(2.1 .4)$ with $p_{0}>0$ we have 


$$
\sum_{n=0}^{\infty} p_{n}<\infty
$$

Finally, every solution $\left\{p_{n}\right\}$ can be written as $p_{n}=u_{n} p_{0}\left(n \in \mathbb{N}_{0}\right)$, where $\left\{u_{n}\right\}_{0}^{\infty}$ denotes the solution of $(2.1 .4)$ with starting value 1 . By choosing $p_{0}=\left\{u_{n}\right\}^{-1}$, the corresponding solution $\left\{p_{n}\right\}_{0}^{\infty}$ becomes a probability distribution.

REMARK. Part (i) of the preceding lemma enables us to speak of the sequence $\left\{r_{n}^{*}(\alpha)\right\}_{0}^{\infty}$, and its $g f R_{\alpha}^{*}$, corresponding to a given lattice distribution $\left\{p_{n}\right\}_{0}^{\infty}$ with $p_{0}>0$. In the sequel we shall do so without further comment.

part (ii) enables us to construct examples of distributions in $C_{\alpha}^{*}$ by starting from an arbitrary sequence $\left\{r_{n}^{*}(\alpha)\right\}_{0}^{\infty}$ of nonnegative numbers satisfying $(2.1 .7)$ or $(2.1 .8)$.

In order to obtain a classification of the inf div lattice distributions, we wish to choose the sequence $\left\{c_{n}^{*}(\alpha)\right\}$ in such a way that

$$
\text { (2.1.9) } \quad C_{\alpha}^{*} \subset C_{\beta}^{*} \text { iff } \alpha<\beta \quad(0 \leq \alpha<1,0<\beta \leq 1)
$$

and we shall say that in this case $\left\{\mathrm{c}_{\mathrm{n}}^{*}(\alpha)\right\}$ satisfies $(2.1 .9)$. In the next section we shall investigate if sequences satisfying (2.1.9) do indeed exist. Here we give a few more properties that hold for every choice of $\left\{c_{n}^{*}(a)\right\}$.

THEOREM 2.1.3. Let $\left\{p_{n}\right\} \in C_{0}$. Then the $r_{n}^{*}(\alpha)^{\text {'s }}$. corresponding to $\left\{p_{n}\right\}$ satisfy

$(2.1 .10) \quad r_{n}^{*}(\alpha) / c_{n}^{*}(\alpha) \geq r_{n}(0) \quad\left(0 \leq \alpha \leq 1 ; n \in \mathbb{N}_{0}\right)$.

Furthermore, if $c_{n+1}^{*}(\alpha) / c_{n}^{*}(\alpha)$ is nondecreasing in $\alpha$ for all $n$, then so are both $r_{n}^{*}(\alpha) / c_{n}^{*}(\alpha)$ and $r_{n}^{*}(\alpha)$.

PROOF. In the first place we note that for the $x_{n}^{*}(\alpha)$ 's corresponding to a general $\left\{p_{n}\right\}$ the following relation holds:

(2.1.11) $\quad \sum_{k=0}^{n} c_{k}^{*}(\alpha) p_{k+1} x_{n-k}^{*}(\beta)=\sum_{k=0}^{n} c_{k}^{*}(\beta) p_{k+1} x_{n-k}^{*}(\alpha) \quad\left(0 \leq \alpha, \beta \leq 1 ; n \in \mathbb{N}_{0}\right)$

in fact, this is a consequence of the following relation (cF. $(2.1 .6)$ ):

$$
A_{\alpha}^{*}(z) R_{\beta}^{*}(z)=A_{\beta}^{*}(z) R_{\alpha}^{*}(z) \quad\left(=P(z) R_{\alpha}^{*}(z) R_{\beta}^{*}(z)\right)
$$


Now, let $\left\{p_{n}\right\} \in C_{0}$, so $r_{n}(0) \geq 0$ for all $n$. Then, applying (2.1.11) with $\beta=0$, we can write

$$
\begin{aligned}
p_{0} r_{n}^{*}(\alpha) & =c_{n}^{*}(\alpha) p_{n+1}-\sum_{k=0}^{n-1} p_{k+1} r_{n-1-k}^{*}(\alpha)= \\
& =c_{n}^{*}(\alpha) p_{n+1}-\sum_{k=0}^{n-1} c_{k}^{*}(\alpha) p_{k+1} r_{n-1-k}(0)
\end{aligned}
$$

so that

$$
\begin{aligned}
& p_{0}\left\{r_{n}^{*}(\alpha)-c_{n}^{*}(\alpha) r_{n}(0)\right\}= \\
& =c_{n}^{*}(\alpha) \sum_{k=0}^{n-1} p_{k+1} r_{n-1-k}(0)-\sum_{k=0}^{n-1} c_{k}^{*}(\alpha) p_{k+1} r_{n-1-k}(0)= \\
& =\sum_{k=0}^{n-1} p_{k+1} r_{n-1-k}(0)\left\{c_{n}^{*}(\alpha)-c_{k}^{*}(\alpha)\right\} .
\end{aligned}
$$

Now by the monotonicity of $c_{n}^{*}(\alpha)$ it follows that $(2.1 .10)$ holds.

Next, let us consider sequences $\left\{c_{n}^{*}(\alpha)\right\}$ for which $c_{n+1}^{*}(\alpha) / c_{n}^{*}(\alpha)$ is nonde-creasing in $\alpha$ for all $\mathrm{n}$, or, equivalently,

$$
(2.1 .12) \quad c_{n}^{*}(\beta) c_{k}^{*}(\alpha) \geq c_{n}^{*}(\alpha) c_{k}^{*}(\beta) \quad\left(0 \leq \alpha<\beta \leq 1 ; n \in \mathbb{N N}_{0} ; k \in\{0,1, \ldots, n\}\right) .
$$

Applying first $(2.1 .4)$ and then twice (2.1.11) with $\beta=0$ and $\alpha=0$, respectively, we can write

$$
\begin{aligned}
& p_{0}\left\{c_{n}^{*}(\alpha) x_{n}^{*}(\beta)-c_{n}^{*}(\beta) x_{n}^{*}(\alpha)\right\}= \\
& =c_{n}^{*}(\beta) \sum_{k=0}^{n-1} p_{k+1} x_{n-1-k}^{*}(\alpha)-c_{n}^{*}(\alpha) \sum_{k=0}^{n-1} p_{k+1} x_{n-1-k}^{*}(\beta)= \\
& =\sum_{k=0}^{n-1} p_{k+1}{ }_{n-1-k}(0)\left\{c_{n}^{*}(\beta) c_{k}^{*}(\alpha)-c_{n}^{*}(\alpha) c_{k}^{*}(\beta)\right\}
\end{aligned}
$$

which is nonnegative on account of (2.1.12) for $\alpha<\beta$. It follows that $x_{n}^{*}(\alpha) / c_{n}^{*}(\alpha)$ is nondecreasing in $\alpha$ for all $n$. Finally, from this we obtain the same property for $r_{n}^{*}(\alpha)$, using the monotonicity of $c_{n}^{*}(\alpha)$ in $\alpha$.

Erom the preceding theorem it follows that definition 2.1.1 can only generate classes $C_{\alpha}^{*}$ with $C_{0}$ as a subclass, we state this as a corollary.

COROLLARY 2.1.4. For every choice of $\left\{c_{n}^{*}(\alpha)\right\}$ and for all $\alpha \in[0,1]: C_{0} c C_{\alpha}^{*}$. 
To conclude this section we prove a simple property of $C_{\alpha}^{*}$, which is already known for $\alpha=0$ and $\alpha=1$.

THEOREM 2.1.5. If $0 \leq \alpha \leq 1$ and $\left\{p_{n}\right\} \in C_{\alpha}^{*}$, then $\left\{p_{n}^{(\gamma)}\right\}_{0}^{\infty} \in C_{\alpha}^{*}$ for all $\gamma \in(0,1)$, where

$$
p_{n}^{(\gamma)}:=\gamma^{n} p_{n} / p(\gamma) \quad\left(n \in \mathbb{N}_{0}\right)
$$

PROOF. It is easy to see that, if $\left\{p_{n}\right\}$ satisfies the recurrence relations $(2.1 .4)$, then $\left\{p_{n}^{(\gamma)}\right\}$ satisfies (2.1.4) with $r_{n}^{*}(\alpha)$ replaced by $\gamma^{n+1} r_{n}^{*}(\alpha)$. As these quantities are nonnegative if the $r_{n}^{*}(\alpha)$ 's are, the theorem follows.

2.2. The choice of $c_{n}^{*}(\alpha)$

clearly, there are many sequences $\left\{c_{n}^{*}(\alpha)\right\}_{0}^{\infty}$ satisfying (2.1.3). Examples of such sequences are easily obtained by a simple interpolation between $c_{n}^{*}(0)=1$ and $c_{n}^{*}(1)=n+1$. Rather more sophisticated, one may try to interpolate between $C_{0}^{*}(z)=(1-z)^{-1}$ and $c_{1}^{*}(z)=(1-z)^{-2}$, where $C_{\alpha}^{*}$ denotes the gf of $\left\{c_{n}^{*}(\alpha)\right\}$. Of both methods we give three examples.

EXAMPLE 2.2.1. The following sequences $\left\{c_{n}^{*}(\alpha)\right\}$ are easily seen to satisfy $(2.1 .3):$

$$
c_{n}^{*}(\alpha)=1+\alpha n
$$

$$
c_{n}^{*}(\alpha)=(1+n)^{\alpha} \text {, }
$$

$$
c_{n 1}^{*}(\alpha)=(1+\alpha n)^{\alpha} \text {. }
$$

$$
c_{\alpha}^{*}(z)=(1-z)^{-1-\alpha} \text {, so } c_{n}^{*}(\alpha)=\left(\begin{array}{c}
\alpha+n \\
n
\end{array}\right) \text {, }
$$

$$
c_{\alpha}^{*}(z)=(1-z)^{-1}(1-\alpha z)^{-1} \text {, so } c_{n}^{*}(\alpha)=1+\alpha+\ldots+\alpha^{n}=\left(1-\alpha^{n+1}\right) /(1-\alpha) \text {, }
$$

$$
c_{\alpha}^{*}(z)=(1-z)^{-1}(1-\alpha z)^{-\alpha} \text {, so } c_{n}^{*}(\alpha)=\sum_{k=0}^{n}\left(\begin{array}{c}
\alpha+k-1 \\
k
\end{array}\right) \alpha^{k} \text {. }
$$

Next we want to investigate which (if any) of the sequences $\left\{c_{n}^{*}(\alpha)\right\}$, listed above, satisfy the desired monotonicity property (2.1.9). Not able to give useful sufficient conditions for (2.1.9), we look for simple necessary conditions, in order to check them for the $c_{n}^{*}(\alpha)$ 's in example 2.2.1. To this end we introduce a special distribution, which is in a way a cxitical one. 
DEFINITION 2.2.2. For a given sequence $\left\{c_{n}^{*}(\alpha)\right\}$ satisfying (2.1.3), the boundary distribution with parameters $\alpha \in[0,1]$ and $\gamma \in\left(0, c^{*}(\alpha)\right)$ is the lattice distribution $\left\{\tilde{\mathrm{p}}_{n}\right\}^{\infty}{ }^{\infty}$, defined by

(2.2.1) $\quad \tilde{p}_{n}=\tilde{p}_{o} \gamma^{n} \prod_{k=0}^{n-1} c_{k}^{*}(\alpha)^{-1} \quad(n \in \mathbb{N})$.

This distribution $\left\{\tilde{p}_{n}\right\}$ can be obtained by choosing in lemma 2.1.2(ii)

$(2.2 .2) \quad x_{0}^{*}(\alpha)=\gamma, x_{n}^{*}(\alpha)=0 \quad(n \in \mathbb{N})$,

which (see also example 3 on p. 57) explains the name "boundary distribution". It follows that

$$
(2.2 .3) \quad\left\{\tilde{p}_{n}\right\}_{0}^{\infty} \in C_{\alpha}^{*}
$$

Computing the $r_{n}^{*}(\alpha)^{\prime}$ 's corresponding to the boundary distribution $\left\{\widetilde{p}_{n}\right\}_{0}^{\infty}$ with parameters $\alpha_{0}$ and $\gamma$, we obtain

$$
r_{n}^{*}(\alpha)=c_{n}^{*}(\alpha) \gamma^{n+1} \prod_{\ell=0}^{n} c_{l}^{*}\left(\alpha_{0}\right)^{-1}-\sum_{k=1}^{n} r_{n-k}^{*}(\alpha) \gamma^{k} \prod_{\ell=0}^{k-1} c_{\ell}^{*}\left(\alpha_{0}\right)^{-1},
$$

from which it successively follows that

$$
\begin{aligned}
& r_{0}^{*}(\alpha)=\gamma, \\
& r_{1}^{*}(\alpha)=\gamma^{2}\left\{c_{1}^{*}(\alpha) / c_{1}^{*}\left(\alpha_{0}\right)-1\right\}, \\
& r_{2}^{*}(\alpha)=\gamma^{3} c_{1}^{*}\left(\alpha_{0}\right)^{-1}\left\{c_{2}^{*}(\alpha) / c_{2}^{*}\left(\alpha_{0}\right)-c_{1}^{*}(\alpha)+c_{1}^{*}\left(\alpha_{0}\right)-1\right\} .
\end{aligned}
$$

Now, if $\left\{\mathrm{C}_{n}^{*}(\alpha)\right\}$ satisfies $(2.1 .9)$, then, as $\left\{\widetilde{p}_{n}\right\} \in C_{\alpha_{0}^{*}}^{*}$, the $r_{n}^{*}(\alpha)$ 's have to be nonnegative for all $\alpha \in\left[\alpha_{0}, 1\right]$. This condition is satisfied for $n=0$ and $n=1$, but not generally for $n=2$. Working out the conditions

$$
x_{2}^{*}(1) \geq 0 \text { and }\left.\frac{d}{d \alpha} x_{2}^{*}(\alpha)\right|_{\alpha=\alpha_{0}} \geq 0
$$

respectively, we get the following lemma (where $\alpha_{0}$ has been replaced by $\alpha$ ).

LEMMA 2.2.3. For $\left\{\mathrm{c}_{\mathrm{n}}^{*}(\alpha)\right\}$ to satisfy (2.1.9) the following conditions are necessary:

(2.2.4) $\quad c_{1}^{*}(\alpha) c_{2}^{*}(\alpha) \geq 3\left(c_{2}^{*}(\alpha)-1\right) \quad(0<\alpha<1)$

and 
(2.2.5) $\quad \frac{\mathrm{d}}{\mathrm{d} \alpha} c_{2}^{*}(\alpha) \geq \mathrm{c}_{2}^{*}(\alpha) \frac{\mathrm{d}}{\mathrm{d} \alpha} c_{1}^{*}(\alpha) \quad(0<\alpha<1)$,

where in the latter case $c_{1}^{*}$ and $c_{2}^{*}$ are supposed to be differentiable.

For the examples of $\left\{\mathrm{c}_{\mathrm{n}}^{*}(\alpha)\right\}$ given in example 2.2 .1 it is not difficult to check whether or not they satisfy $(2.2 .4)$ and $(2.2 .5)$. Therefore we state the mostly negative results without proofs.

LEMPA 2.2 .4 .

(i) $c_{n}^{*}(\alpha)=1+$ an violates $(2.2 .4)$ iff $\frac{3}{2}<\alpha<1$, and (2.2.5) iff $\frac{1}{2}<\alpha<1$.

(ii) $\quad c_{n}^{*}(\alpha)=(1+n)^{\alpha}$ violates $(2.2 .4)$ if $0.7427<\alpha<1$, and $(2.2 .5)$ iff ${ }^{2} \log ^{2} \log 3(\simeq 0.6645)<\alpha<1$.

(iii) $c_{n}^{*}(\alpha)=(1+\alpha n)^{\alpha}$ violates $(2.2 .5)$ if $0.7573<\alpha<1$.

(iv) $c_{n}^{*}(\alpha)=\left(\begin{array}{c}\alpha+n \\ n\end{array}\right)$ violates $(2.2 .4)$ iff $\sqrt{3}-1(\simeq 0.7321)<\alpha<1$, and $(2.2 .5)$ iff $1_{2}(\sqrt{5}-1)(\simeq 0.6180)<\alpha<1$.

(v) $\quad c_{n}^{*}(\alpha)=\left(1-\alpha^{n+1}\right) /(1-\alpha)$ satisfies both $(2.2 .4)$ and $(2.2 .5)$.

(vi) $c_{n}^{*}(\alpha)=\sum_{k=0}^{n}\left(\begin{array}{c}\alpha+k-1 \\ k\end{array}\right) \alpha^{k}$ violates $(2.2 .4)$ if $0.9344<\alpha<1$, and $(2.2 .5)$ i.f $0.8907<\alpha<1$.

It is a pity that $(1+n)^{\alpha}$ and $\left(\begin{array}{c}\alpha+n \\ n\end{array}\right)\left(\sim n^{\alpha} / \Gamma(\alpha+1), n \rightarrow \infty\right)$ do not have the required properties, as they seem to be more interesting, especially with regard to asymptotic behaviour, than the only choice left: $c_{n}^{*}(\alpha)=\left(1-\alpha^{n+1}\right) /$ $(1-\alpha)$, for which we have $c_{n}^{*}(\alpha) \sim(1-\alpha)^{-1}(n \rightarrow \infty)$. It would be interesting to know whethex other choices of $\left\{c_{n}^{*}(\alpha)\right\}$ exist, satisfying (2.1.3) and $(2.1 .9)$, with a more attractive limit behaviour, e.g. $c_{n}^{*}(\alpha) \sim n^{\alpha}(n \rightarrow \infty)$. It might be possible to obtain more interesting $c_{n}^{*}(\alpha)$ 's by weakening somewhat the condition (2.1.3). Dropping, for example, the monotonicity of $c_{0}^{*}(\alpha)$, we may consider $c_{n}^{*}(\alpha)^{\prime}$ s like $(n+\alpha)^{\alpha}$ and the one obtained by means of "fractional differentiation" as follows:

$$
\begin{aligned}
C_{\alpha}^{*}(z) & =z^{\alpha-1}\left(\frac{d}{d z}\right)^{\alpha}\left[z(1-z)^{-1}\right]=z^{\alpha-1}\left(\frac{d}{d z}\right)^{\alpha}\left[\sum_{n=1}^{\infty} z^{n}\right]= \\
& =z^{\alpha-1} \sum_{n=1}^{\infty} \frac{\Gamma(n+1)}{\Gamma(n-\alpha+1)} z^{n-\alpha}
\end{aligned}
$$

so 


$$
\text { (2.2.6) } \quad c_{n}^{*}(\alpha)=\frac{(n+1) !}{\Gamma(n+2-\alpha)}=\frac{1}{\Gamma(1-\alpha)}\left(\begin{array}{c}
n+1-\alpha \\
n+1
\end{array}\right)^{-1} \text {. }
$$

It turns out, however, that also these two choices fail to satisfy $(2.1 .9)$; if $c_{n}^{*}(\alpha)=(n+\alpha)^{\alpha}$, then computing ${ }^{1)} r_{3}^{*}(1)$ corresponding to the boundary distribution $\left\{\tilde{p}_{n}\right\}_{0}^{\infty}$ with parameters $\alpha$ and $\gamma$, we obtain, for instance for $\alpha=0.6$ and $\gamma=1: r_{3}^{*}(1) \simeq-0.0089$; if $c_{n}^{*}(\alpha)$ is given by $(2.2 .6)$, then $r_{2}^{*}(1)$, corresponding to $\left\{\tilde{p}_{n}\right\}$, turns out to satisfy: $r_{2}^{*}(1)<0$ iff $3-\sqrt{5}(\simeq 0.7639)$ $<\alpha<1$.

Although (2.2.6) does not satisfy $(2.1 .9)$, it suggests a class of other possible choices of $c_{n}^{*}(\alpha)$. If we rewrite $(2.2 .6)$ as

$$
(2.2 .7) \quad c_{n}^{*}(\alpha)=\frac{n+1}{\Gamma(1-\alpha)} \int_{(0,1)}(1-u)^{-\alpha} u^{n} d u \quad\left(n \in \mathbb{N}_{0} ; 0 \leq \alpha<1\right) \text {, }
$$

then by (2.1.4) it follows that the $g f \mathrm{R}_{\alpha}^{*}$ of $\left\{\mathrm{r}_{\mathrm{n}}^{*}(\alpha)\right\}_{0}^{\infty}$, corresponding to a given lattice distribution $\left\{\mathrm{p}_{\mathrm{n}}\right\}^{\infty}{ }^{\prime}$ is given by

$$
\text { (2.2.8) } \quad R_{\alpha}^{*}(z)=\frac{1}{\Gamma(1-\alpha)} \int_{(0,1)}(1-u)^{-\alpha} \frac{P^{*}(u z)}{P(z)} d u \quad(0 \leq \alpha<1) \text {. }
$$

Now $(2.2 .7)$ and $(2.2 .8)$ can be generalized in the following way:

$$
\text { (2.2.9) } \quad c_{n}^{*}(\alpha)=c_{0}^{*}(\alpha)(n+1) \int_{(0,1)} g_{\alpha}(u) u^{n} d u \quad\left(n \in \mathbb{N}_{0} ; 0 \leq \alpha<1\right)
$$

and

$$
(2.2 .10) \quad R_{\alpha}^{*}(z)=c_{0}^{*}(\alpha) \int_{(0,1)} g_{\alpha}(u) \frac{P^{\prime}(u z)}{P(z)} d u \quad(0 \leq \alpha<1),
$$

where for all $\alpha \in[0,1) g_{\alpha}$ is a pdf on $(0,1)$ with the property that

$$
g_{0}(u)=1_{(0,1)}(u) \text { and } \lim _{\alpha \uparrow 1} g_{\alpha}(u)=\delta_{1}(u)
$$

$\left(\delta_{1}\right.$ is Dirac's delta function at 1$)$. It is not clear though, under what conditions $R_{\beta}^{*}$ would be abs mon if $R_{\alpha}^{*}$ is abs mon and $\alpha<\beta$. Rather curiously, as

$$
\frac{1-\alpha^{n+1}}{1-\alpha}=(n+1) \int_{(\alpha, 1)} \frac{1}{1-\alpha} u^{n} d u \quad\left(n \in \mathbb{N}_{0} ; 0 \leq \alpha<1\right),
$$

1) My thanks are due to L.G.F.C. van Bree, who did the programming needed here and in some other cases. 
the only remaining example of $c_{n}^{*}(\alpha)$ from lemma 2.2 .4$, i.e. $c_{n}^{*}(\alpha)=$ $=\left(1-\alpha^{n+1}\right) /(1-\alpha)$, is of the form (2.2.9) with $g_{\alpha}$ the uniform pdf on $(\alpha, 1)$. We shall not pursue this in this monograph.

We now restrict our attention to the only remaining candidate, $c_{n}^{*}(\alpha)=$ $=\left(1-\alpha^{n+1}\right) /(1-\alpha)$. This choice, which we shall consider in detail in the next section, is supported by the following lemma.

LEMMA 2.2.5. If $c_{1}^{*}(\alpha)$ and $c_{2}^{*}(\alpha)$ are linear and quadratic functions, respectively, then for $(2.2 .4)$ and $(2.2 .5)$ to hold it is necessary and sufficient that

$$
c_{1}^{*}(\alpha)=1+\alpha \cdot c_{2}^{*}(\alpha)=1+\alpha+\alpha^{2} \quad(0 \leq \alpha \leq 1) .
$$

PROOF. Because of $(2.1 .3) \mathrm{c}_{1}^{*}$ and $\mathrm{c}_{2}^{*}$ have necessarily the form

$$
c_{1}^{*}(\alpha)=1+\alpha, c_{2}^{*}(\alpha)=1+(2-b) \alpha+b \alpha^{2} \text {, }
$$

where $-2 \leq b \leq 1, b \neq 0$. The sufficiency of the condition has already been shown in lemma 2.2.4. Suppose therefore that (2.2.4) holds. Then it is easy to see that

$$
b \geq(2 \alpha-1) /\{\alpha(2-\alpha)\} \quad(0 \leq \alpha \leq 1),
$$

from which it follows that $b \geq 1$. Hence $b=1$, and the lemma is proved.

2.3. The classes $C_{\alpha}$; basic properties

From now on we shall consider the $c_{n}^{*}(\alpha)$ from lemma $2.2 .4(v)$, and we shall show that it satisfies indeed (2.1.9). For this special case we use the nom tation without $*$, so we have

$$
c_{n}(\alpha)=\frac{1-\alpha^{n+1}}{1-\alpha}=\sum_{k=0}^{n} \alpha^{k} \quad\left(n \in \mathbb{N}_{0} ; 0 \leq \alpha \leq 1\right)
$$

while the corresponding classes $C_{\alpha}$ are defined as follows.

DEEINITION 2.3.1. A lattice distribution $\left\{\mathrm{p}_{\mathrm{n}}\right\}^{\infty}{ }^{\infty}$ with $\mathrm{p}_{0}>0$, is said to be in the class $C_{\alpha}$ if there exist $r_{n}(\alpha) \geq 0\left(n \in \mathbb{N}_{0}\right)$ such that

(2.3.1) $\quad \frac{1-\alpha^{n+1}}{1-\alpha} p_{n+1}=\sum_{k=0}^{n} p_{k}{ }_{n-k}(\alpha) \quad\left(n \in \mathbb{N}_{0}\right)$. 
Applying lemma 2.1.2 to our special situation, we easily obtain the following properties of (2.3.1). We only note that $c(\alpha)$ and $A_{\alpha}$, defined by $(2.1 .5)$ and $(2.1 .6)$, respectively, are now given by

$$
C(\alpha)=\frac{1}{1-\alpha}, A_{\alpha}(z)=\frac{P(z)-P(\alpha z)}{(1-\alpha) z} \quad(0 \leq \alpha<1 ;|z| \leq 1) .
$$

LEMMA 2.3.2. Consider the recurrence relations (2.3.1) for a fixed $\alpha \in[0,1)$.

(i) For every lattice distribution $\left\{\mathrm{p}_{\mathrm{n}}\right\}_{0}^{\infty}$ with $\mathrm{p}_{\mathrm{o}}>0$ there exists a unique sequence $\left\{r_{n}(\alpha)\right\}_{0}^{\infty}$ satisfying $(2.3 .1)$. The $g f R_{\alpha}$ of $\left\{r_{n}(\alpha)\right\}$ has a positive radius of convergence, while in a neighbourhood of zero

$$
(1-\alpha) z R_{\alpha}(z)=1-P(\alpha z) / P(z) .
$$

Furthermore, if $r_{n}(\alpha) \geq 0$ for all $n$ (i.e. if $\left\{p_{n}\right\} \in C_{\alpha}$ ), then

$$
\text { (2.3.3) } \sum_{n=0}^{\infty} x_{n}(\alpha)<\frac{1}{1-\alpha} \text {. }
$$

(ii) For every sequence $\left\{r_{n}(\alpha)\right\}_{0}^{\infty}$ with $r_{n}(\alpha) \geq 0$ for all $n$ and satisfying $(2.3 .3)$ there exists a unique lattice distribution $\left\{p_{n}\right\}_{0}^{\infty}$ with $p_{0}>0$, satisfying $(2.3 .1)$.

As noted in section 1, we shall speak of the sequence $\left\{x_{n}(\alpha)\right\}$ corresponding to $\left\{p_{n}\right\}$, when considering the solution of (2.3.1) for $\left\{r_{n}(\alpha)\right\}$. We shall refer to its $g f R_{\alpha}$ as the $R_{\alpha}$--function of $\left\{p_{n}\right\}$ or $P$. It is now obvious that we have the following useful characterization of $C_{\alpha}$ in terms of pgf's (cf. definition 1.3.1).

LEMMA 2.3.3. For $0 \leq \alpha<1$ a pgf $P$, with $P(0)>0$, is in $C_{\alpha}$ iff the $R_{\alpha}$-function of $P$, given by $(2.3 .2)$, is abs mon.

Taking $\alpha=0$ in the preceding lemma, we get theorem 1.5.8, while the first part of theorem 1.5 .3 is a limiting case in the following sense:

$$
\lim _{\alpha \uparrow 1} R_{\alpha}(z)=\lim _{\alpha \uparrow 1} \frac{1}{P(z)} \frac{P(z)-P(\alpha z)}{z-\alpha z}=\frac{P^{\prime}(z)}{P(z)}=R_{1}(z)
$$

As an illustration of the usefulness of lemma 2.3 .3 we prove corollary 2.1.4 in our situation using gf's. Rewriting (2.3.2) for $\alpha=0$ as

$$
\text { (2.3.4) } P(z)=P(0) /\left\{1-z R_{0}(z)\right\},
$$


and substituting this in (2.3.2), we obtain for $0<\alpha<1$ the following relation between the $R_{0}-$ and $R_{\alpha}$-function of a pgf $P$ :

$$
(2.3 .5) \quad(1-\alpha) R_{\alpha}(z)=\left\{R_{0}(z)-\alpha R_{0}(\alpha z)\right\} /\left\{1-\alpha z R_{0}(\alpha z)\right\} .
$$

It follows that $R_{\alpha}$ is abs mon if $R_{0}$ is abs mon. Hence we have proved

$$
C_{0} \subset C_{\alpha} \quad(0 \leq \alpha \leq 1) .
$$

We now turn to the general monotonicity property (2.1.9). As a first step we prove that for every $\alpha \in[0,1)$ the distributions in $C_{\alpha}$ are inf div.

THEOREM 2.3.4. FOr all $\alpha \in[0,1)$ the following inclusion holds:

$$
C_{\alpha} \subset C_{1}
$$

PROOF. Let $0 \leq \alpha<1$ and $P \in C_{\alpha}$. Then according to (2.3.2) and (2.3.3) for $|z| \leq 1$ we can write

$$
(2.3 .6) \quad P(z)=\left\{1-(1-\alpha) z_{\alpha}(z)\right\}^{-1} P(\alpha z) .
$$

Iterating this equation we obtain for every $n \in \mathbb{N}$

$$
P(z)=P\left(\alpha^{n} z\right) \prod_{k=0}^{n-1}\left\{1-(1-\alpha) \alpha^{k}{ }_{\alpha R}\left(\alpha^{k} z\right)\right\}^{-1} \quad(|z| \leq 1) .
$$

Taking $z=1$ we see that

$$
P\left(\alpha^{n}\right)=\prod_{k=0}^{n-1}\left\{1-(1-\alpha) \alpha^{k} R_{\alpha}\left(\alpha^{k}\right)\right\}
$$

and so

$$
P(z)=\frac{P\left(\alpha^{n} z\right)}{P\left(\alpha^{n}\right)} \prod_{k=0}^{n-1} \frac{1-(1-\alpha) \alpha^{k} R_{\alpha}\left(\alpha^{k}\right)}{1-(1-\alpha) \alpha^{k} z R_{\alpha}\left(\alpha^{k} z\right)} \quad(|z| \leq 1 ; n \in \mathbb{N}) .
$$

From this, by letting $n \rightarrow \infty$, the following expression for $P$ in $R_{\alpha}$ is $o b-$ tained:

$$
\text { (2.3.7) } P(z)=\prod_{k=0}^{\infty} \frac{1-(1-\alpha) \alpha^{k} R_{\alpha}\left(\alpha^{k}\right)}{1-(1-\alpha) \alpha^{k} z R_{\alpha}\left(\alpha^{k} z\right)} \quad(|z| \leq 1),
$$

where it is easily verified that the infinite product is absolutely conver- 
gent. Now define $\pi_{k}:=(1-\alpha) \alpha^{k} R_{\alpha}\left(\alpha^{k}\right)$ and $Q_{k}(z):=z R_{\alpha}\left(\alpha^{k} z\right) / R_{\alpha}\left(\alpha^{k}\right)$. Then $P$ takes the form

$$
P(z)=\prod_{k=0}^{\infty} \frac{1-\pi_{k}}{1-\pi_{k} Q_{k}(z)} \quad(|z| \leq 1),
$$

where $\pi_{k} \in[0,1)$ because of $(2.3 .3)$ and $\Omega_{k}$ is a pgf because of the abs mon of $R_{\alpha}$ (lemma 2.3.3). It follows that $P$ is the limit of a sequence of products of pgf's in $\mathcal{C}_{0} \subset \mathcal{C}_{1}$, and hence by theorems 1.4 .2 and 1.4 .3 we conclude that $P \in C_{1}$.

Equation (2.3.7) entails a representation theorem for $\mathcal{C}_{\alpha}$ (cf. the representations $(1.5 .1)$ and $(1.5 .12)$ for $C_{1}$ and $C_{0}$, respectively).

THEOREM 2.3.5. For $0<\alpha<1$ a pgf $P$, with $P(0)>0$, is in $C_{\alpha}$ iff $P$ has the form

(2.3.8) $\quad P(z)=\prod_{k=0}^{\infty} \frac{1-p Q\left(\alpha^{k}\right)}{1-p Q\left(\alpha^{k} z\right)}$,

where $p \in(0,1)$ and $Q$ is a pgf with $Q(0)=0$. The representation $(p, Q)$ is unique.

PROOF. If $P \in C_{\alpha}$, then $P$ can be represented by (2.3.7), which takes the form (2.3.8) if we put $p=(1-\alpha) R_{\alpha}(1)$ and $Q(z)=z R_{\alpha}(z) / R_{\alpha}(1)$.

Conversely, a pgf $\mathrm{P}$ of the form $(2.3 .8)$ has an $\mathrm{R}_{\alpha}$-function given by

$$
\text { (2.3.9) } \quad R_{\alpha}(z)=\frac{p}{1-\alpha} \frac{1}{z} Q(z),
$$

which, as $Q(0)=0$, is an abs mon function. Hence by lemma $2.3 .3 p \in C_{\alpha}$. The uniqueness of the representation $(p, 2)$ follows from $(2.3 .9)$ and the uniqueness of $R_{\alpha}$.

In order to prove the general monotonicity property (relation (2.1.9)) by using lemma 2.3.3, we need a convenient relation between the $R_{\alpha}-$ and $R_{B}-$ function of a pgf $P$. Such a relation is rather hard to find, but the following alternative proof of theorem 2.3.4 suggests relation (2.3.13), which is the key to the proof of the main theorem. On account of $(2.3 .2)$ we can write for $0 \leq \alpha<1$ (cf. $(1.5 .2)$ )

$$
(1-\alpha) \frac{d}{d z}\left[z R_{\alpha}(z)\right]=-\frac{d}{d z}[P(\alpha z) / P(z)]=\frac{P(\alpha z)}{P(z)}\left\{R_{1}(z)-\alpha R_{1}(\alpha z)\right\},
$$


and so

(2.3.10) $R_{1}(z)-\alpha R_{1}(\alpha z)=(1-\alpha) \frac{P(z)}{P(\alpha z)} \frac{d}{d z}\left[z R_{\alpha}(z)\right]$.

Now, let $0 \leq \alpha<1$ and $P \in C_{\alpha}$. Then $R_{\alpha}$ is abs mon, which by (2.3.6) implies the abs mon of $P(z) / P(\alpha z)$. It follows that the left-hand side of $(2.3 .10)$, and hence $R_{1}$, is abs mon too, and so $P \in C_{1}$.

REMARK. Equating the coefficients of $z^{n}$ in (2.3.10) we obtain the following analogue of $(2.1 .10)$ : if $\left\{p_{n}\right\} \in C_{\alpha}$, then the $r_{n}(\alpha)^{\prime}$ s corresponding to $\left\{p_{n}\right\}$ satisfy (cf. corollary 2.3 .8 and its proof)

$(2.3 .11) \quad x_{n}(\alpha) / c_{n}(\alpha) \leq x_{n}(1) /(n+1) \quad\left(n \in \mathbb{N}_{0}\right)$.

In the proof of the main theorem we shall need the following lemma.

LEMMA 2.3.6. If $P \in C_{1}$, then $P(z) / P(\gamma z)$ is abs mon for $0 \leq \gamma \leq 1$. In fact, $P(\gamma) P(z) / P(\gamma z)$ is a pge in $C_{1}$.

PROOF. If $P \in C_{1}$, then $P$ has the form (1.5.1), from which it follows that $P(\gamma) P(z) / P(\gamma z)=\exp [\mu\{Q(z)-Q(\gamma z)+Q(\gamma)-1\}]$.

For $0 \leq \gamma \leq 1$ this is again of the form (1.5.1), so $P(\gamma) P(z) / P(\gamma z) \in C_{1}$. $\square$

THEOREM 2.3.7. $C_{\alpha}$ is nondecreasing in $\alpha \in[0,1]$, i.e. for all $\alpha, \beta \in[0,1]$

$$
C_{\alpha} \subset C_{\beta} \text { iff } \alpha<\beta \text {. }
$$

PROOF. The theorem has been already proved in the case $0 \leq \alpha \leq 1, \beta=1$; so, in view of lemma 2.3.3, it remains to show that, if $0 \leq \alpha<\beta<1$ and if $R_{\alpha}$ is abs mon, then $R_{\beta}$ is abs mon. To do so, from (2.3.2) we subtract the same equality with $z$ replaced by $\beta z$, and we obtain

$$
z(1-\alpha)\left\{R_{\alpha}(z)-\beta R_{\alpha}(\beta z)\right\}=\frac{P(\alpha \beta z)}{P(\beta z)}-\frac{P(\alpha z)}{P(z)}
$$

or, dividing by $P(\alpha z)$,

(2.3.12) $\frac{z(1-\alpha)}{\mathrm{P}(\alpha z)}\left\{\mathrm{R}_{\alpha}(z)-\beta \mathrm{R}_{\alpha}(\beta z)\right\}=\frac{\mathrm{P}(\alpha \beta z)}{\mathrm{P}(\alpha z) \mathrm{P}(\beta z)}-\frac{1}{\mathrm{P}(z)}$.

The right-hand side of (2.3.12), and hence the left-hand side of (2.3.12), is symmetric in $\alpha$ and $\beta$, from which it follows that for $0 \leq \alpha<1$ and 
$0 \leq \beta<1$ we have

(2.3.13) $\quad R_{\beta}(z)-\alpha R_{\beta}(\alpha z)=\frac{1-\alpha}{1-\beta} \frac{P(\beta z)}{P(\alpha z)}\left\{R_{\alpha}(z)-\beta R_{\alpha}(\beta z)\right\}$.

Now suppose that $\alpha<\beta$ and $P \in C_{\alpha}$. Then by theorem 2.3.4 P $\in C_{1}$, and from lemma 2.3 .6 we conclude that $P(z) / P(\{\alpha / \beta\} z)$, and so $P(\beta z) / P(\alpha z)$, is abs mon. From the abs mon of $R_{\alpha}$ we obtain the abs mon of $R_{\alpha}(z)-\beta R_{\alpha}(\beta z)$, so that by (2.3.13) it follows that $R_{\beta}(z)-\alpha R_{\beta}(\alpha z)$, with coefficients $\left(1-\alpha^{n+1}\right) x_{n}(\beta)$, is abs mon. Hence $R_{\beta}$ is abs mon, and the theorem is proved.

Looking somewhat more precisely at the identity (2.3.13), we obtain a generalization of the inequalities $(2.1 .10)$ and $(2.3 .11)$.

COROLIARY 2.3.8. If $0 \leq \alpha_{0}<1$ and if $P \in C_{\alpha_{0}}$, then $r_{n}(\alpha) / C_{n}(\alpha)$, and hence $r_{n}(\alpha)$, is nondecreasing in $\alpha \in\left[\alpha_{0}, 1\right]$ for all $n \in \mathbb{N}_{0}$, i.e.

$(2.3 .14) \quad r_{n}(\alpha) / c_{n}(\alpha) \leq x_{n}(\beta) / c_{n}(\beta) \quad\left(n \in \mathbb{N}_{0} ; \alpha_{0} \leq \alpha \leq \beta \leq 1\right)$.

PROOF. Let $P \in \mathcal{C}_{\alpha_{0}}$ and, in view of (2.3.11), $\alpha_{0} \leq \alpha \leq \beta<1$. Then by theorem 2.3.7 also $P \in C_{\alpha}$, and hence ( $C f$. the proof of theorem 2.3.7) $P(\beta z) / P(\alpha z)$ can be written as

$$
P(\beta z) / P(\alpha z)=\sum_{n=0}^{\infty} s_{n}(\alpha, \beta) z^{n}
$$

where $s_{n}(\alpha, \beta) \geq 0$ for all $n$ and $s_{0}(\alpha, \beta)=1$. Now, equating the coefficients of $z^{n}$ in (2.3.13), we see that

$$
c_{n}(\alpha) r_{n}(\beta)=\sum_{k=0}^{n} c_{k}(\beta) r_{k}(\alpha) s_{n-k}(\alpha, \beta) \quad\left(n \in \mathbb{N}_{0}\right),
$$

from which (2.3.14) follows. Finally, as $c_{n}(\alpha) \leq c_{n}(\beta) \quad\left(n \in \mathbb{N}_{0}\right)$, we conclude from $(2.3 .14)$ that $x_{n}(\alpha) \leq r_{n}(\beta) \quad\left(n \in \mathbb{N}_{0}\right)$.

We restate theorem 2.3 .7 as a property that generalizes a result of Goldie (1967), who proved, in fact, our result in case $\alpha=0$ and $\beta=1$.

COROLLARY 2.3.9. If for a given distribution $\left\{p_{n}\right\}_{0}^{\infty}$, with $p_{0}>0$, the quantities $x_{n}(\alpha)$, for a fixed $\alpha \in[0,1)$ and $n \in \mathbb{N}_{0}$ defined by 
$(2.3 .15) \quad\left(1+\alpha+\alpha^{2}+\ldots+\alpha^{n}\right) p_{n+1}=\sum_{k=0}^{n} p_{k}{ }_{n-k}(\alpha) \quad\left(n \in \mathbb{N}_{0}\right)$

are all nonnegative, then also the quantities $x_{n}(\beta)$, defined by $(2.3 .15)$

with $\alpha$ replaced by $\beta$, are nonnegative for all $n \in \mathbb{N}_{0}$ and all $\beta$ with $\alpha<\beta \leq 1$.

\subsection{Further properties and examples}

In view of theorem 2.3.7 the classes $C_{\alpha}$ define a classification (cf. the end of section 1.2) of $C_{1}$. In this section we shall compare the properties of the separate $C_{\alpha}$ 's with those known for $C_{1}$. To this end the characterization of $C_{\alpha}$, given by lemma 2.3 .3 , is frequently used, and, for notational convenience, we denote by $R_{\alpha}^{(\gamma)}$ the $R_{\alpha}$-function of a pgf, $P_{\gamma}$, depending on a parameter $\gamma$.

We start with a generalization of the closure property of $\mathcal{C}_{1}$ (theorem 1.4.3).

THEOREM 2.4.1. For $0 \leq \alpha \leq 1$ the class $C_{\alpha}$ is closed under weak convergence, i.e. a pgf $P$, for which there exist $\operatorname{pgf}^{\prime} s \mathrm{P}_{n} \in C_{\alpha}(n \in \mathbb{N})$ such that $P(z)=$ $=\lim _{n \rightarrow \infty} P_{n}(z)(0 \leq z \leq 1)$, is in $C_{\alpha}$.

PROOF. Let $0 \leq \alpha<1$, and let $P$ be a pgf for which $P(z)=\lim _{n \rightarrow \infty} P_{n}(z)$ with $\mathrm{P}_{\mathrm{n}} \in \mathrm{C}_{\alpha}(\mathrm{n} \in \mathbb{N})$. Then $\mathrm{R}_{\alpha}^{(\mathrm{n})}$ is abs mon for all $\mathrm{n}$, and as on account of $(2.3 .2)$ the $R_{\alpha}$-function of $P$ satisfies $R_{\alpha}(z)=\lim _{n \rightarrow \infty} R_{\alpha}^{(n)}(z)$, it follows by lemma $1.3 .3(\mathrm{iv})$ that $R_{\alpha}$ is abs mon, too. Hence $P \in C_{\alpha}$.

In the following theorem we state some properties of $C_{\alpha}$ that are well known,

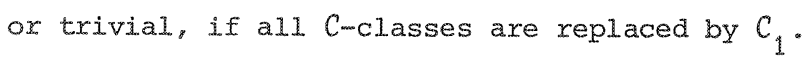

THEOREM 2.4.2. For $0 \leq \alpha \leq 1$ the class $C_{\alpha}$ has the following properties.

(i) If $\mathrm{P} \in \mathcal{C}_{\alpha}$ and $0 \leq \gamma \leq 1$, then $P(\gamma z) / P(\gamma) \in C_{\alpha}$.

(ii) If $\mathrm{P} \in \mathrm{C}_{\alpha}$ and $0 \leq \gamma \leq 1$, then $\mathrm{P}(\mathrm{z})^{\gamma} \in \mathrm{C}_{\alpha}$.

(iii) If $P \in C_{\alpha}$ and $0 \leq \gamma \leq 1$, then $P_{\gamma}(z):=P(\gamma) P(z) / P(\gamma z) \in C_{\alpha}$.

(iv) If $P \in C_{\alpha}, n \in \mathbb{N}$ and $\alpha^{1 / n} \leq \gamma \leq 1$, then

(2.4.1) $\quad P_{n, \gamma}(z):=\prod_{k=0}^{n-1} P\left(\gamma^{k} z\right) / P\left(\gamma^{k}\right) \in C_{\gamma}$. 
PROOF. In all four cases we apply lemma 2.3.3.

(i) In fact, this result has already been proved in theorem 2.1.5, but it also follows from the following obvious relation:

(2.4.2) $\quad R_{\alpha}^{(\gamma)}(z)=\gamma R_{\alpha}(\gamma z)$

(ii) For the $R_{\alpha}$-function $R_{\alpha}^{(\gamma)}$ of $\mathrm{P}^{\gamma}$ we have

$$
\begin{aligned}
\frac{\mathrm{d}}{\mathrm{d} z}\left[(1-\alpha) z_{\alpha}(\gamma)(z)\right] & =-\frac{\mathrm{d}}{\mathrm{dz}}\left[(P(\alpha z) / P(z))^{\gamma}\right]= \\
& =-\gamma(P(\alpha z) / P(z))^{\gamma-1} \frac{\mathrm{d}}{\mathrm{dz}}[P(\alpha z) / P(z)] .
\end{aligned}
$$

and so

(2.4.3) $\quad \frac{d}{d z}\left[z R_{\alpha}^{(\gamma)}(z)\right]=\gamma(P(z) / P(\alpha z))^{1-\gamma} \frac{d}{d z}\left[z R_{\alpha}(z)\right]$.

Now, if $P \in C_{\alpha}$, then $P \in C_{1}$, and by lemma 2.3 .6 we know that $P(\alpha) P(z) / P(\alpha z)$, and hence $\{P(\alpha) P(z) / P(\alpha z)\}^{1-\gamma}$ for $0 \leq \gamma \leq 1$, is a pgf in $C_{1}$. It follows that $\{P(z) / P(\alpha z)\}^{1-\gamma}$ is abs mon for $0 \leq \gamma \leq 1$. As $R_{\alpha}$ is abs mon too, we $o b-$ tain the abs mon of $R_{\alpha}^{(\gamma)}$ from (2.4.3).

(iii) First, note that, if $P \in C_{\alpha}$ then for $0 \leq \gamma \leq 1 P_{\gamma}$ is indeed a pgf (cf. lemma 2.3.6). Calculating the $R_{\alpha}$-function of $P_{\gamma}$ we obtain

$$
\left(1-\alpha_{0}\right) z R_{\alpha}^{(\gamma)}(z)=1-\frac{P(\alpha z)}{P(\alpha \gamma z)} \frac{P(\gamma z)}{P(z)}=\frac{P(\gamma z)}{P(\alpha \gamma z)}\left\{\frac{P(\alpha \gamma z)}{P(\gamma z)}-\frac{P(\alpha z)}{P(z)}\right\},
$$

and so

$(2.4 .4) \quad R_{\alpha}^{(\gamma)}(z)=\frac{P(\gamma z)}{P(\alpha \gamma z)}\left\{R_{\alpha}(z)-\gamma R_{\alpha}(\gamma z)\right\}$.

As according to lemma 2.3.6 $P(\gamma z) / P(\alpha \gamma z)$ is abs mon, we obtain the abs mon of $R_{\alpha}^{(\gamma)}$ from that of $R_{\alpha}$, using $(2.4 .4)$.

(iv) We calculate the $R_{\gamma}$-function of $P_{n, \gamma}$, and we get

$(2.4 .5) \quad R_{\gamma}^{(n, \gamma)}(z)=c_{n-1}(\gamma) R_{\gamma}(z)$.

By hypothesis, this is abs mon for $\gamma^{n}=\alpha$, and hence by theorem 2.3 .7 for $\gamma^{n} \geq \alpha$

Taking $\alpha=0$ in part (iv) of the preceding theorem, we see that for every $n \in \mathbb{N}$ the product of the first $n$ factors in the canonical representation (2.3.8) of a pgf in $C_{\alpha}$ also is a pgf in $C_{\alpha}$. We state this as a corollary. 
COROLIARY 2.4.3. If $0 \leq \alpha \leq 1, \mathrm{n} \in \mathbb{N}, 0 \leq \mathrm{p}<1$ and if $\mathrm{Q}$ is a pgf with $Q(0)=0$, then

$$
(2.4 .6) \prod_{k=0}^{n-1} \frac{1-p Q\left(\alpha^{k}\right)}{1-p Q\left(\alpha^{k} z\right)} \in C_{\alpha} .
$$

Next we prove a property of the $C_{\alpha}{ }^{\prime}$ 's, showing that, though many inf div distributions are in $C_{1} \backslash \underset{0 \leq \alpha<1}{U} C_{\alpha}$ (cf. example 2 on p.56), the situation is not too bad: we prove that $\underset{0 \leq \alpha<1}{U} C_{\alpha}$ is dense in $C_{1}$ in the sense of weak convergence.

THEOREM 2.4.4. If $P \in C_{1}$, then there exist an increasing sequence $\left\{\alpha_{n}\right\}_{1}^{\infty}$, with $\lim _{n \rightarrow \infty} \alpha_{n}=1$, and a sequence of $\operatorname{pgf}^{\prime \prime} P_{n} \in \mathcal{C}_{\alpha_{n}}(n \in \mathbb{N})$, such that

$$
(2.4 .7) \quad P(z)=\lim _{n \rightarrow \infty} P_{n}(z) \quad(|z| \leq 1) .
$$

PROOF. Let $P \in C_{1}$. Then by theorem 1.5.1 $P$ has the form (2.4.8) $P(z)=\exp [\mu(Q(z)-1)]$. where $\mu>0$ and $Q$ is a pgf with $Q(0)=0$. Take $\alpha_{n}=1-n^{-2}$, and for $n>\mu$ define the pgf's $P_{n}$ by

$$
P_{n}(z):=\prod_{k=0}^{n-1} \frac{1-\{\mu / n\} Q\left(\alpha_{n}^{k}\right)}{1-\{\mu / n\} Q\left(\alpha_{n}^{k} z\right)} .
$$

From corollaxy 2.4 .3 we know that $\mathrm{P}_{\mathrm{n}} \in \mathrm{C}_{\alpha_{\mathrm{n}}}$. We rewrite $\mathrm{P}_{\mathrm{n}}$ as

$$
\text { (2.4.9) } \quad P_{n}(z)=\left\{1+\frac{\mu}{n}(Q(z)-1)\right\}^{n} \prod_{k=0}^{n-1}\left\{1+g_{k, n}(z)\right\} \text {, }
$$

with

$$
\begin{aligned}
g_{k, n}(z): & =\frac{n-\mu Q\left(\alpha_{n}^{k}\right)}{n-\mu Q\left(\alpha_{n}^{k} z\right)} \frac{1}{1+\{\mu / n\}(Q(z)-1)}-1= \\
& =\mu \frac{1-Q\left(\alpha_{n}^{k}\right)+Q\left(\alpha_{n}^{k} z\right)-Q(z)+\{\mu / n\} Q\left(\alpha{ }_{n}^{k} z\right)(Q(z)-1)}{\left[n-\mu Q\left(\alpha_{n}^{k} z\right)\right][1+\{\mu / n\}(Q(z)-1)]} .
\end{aligned}
$$

It follows that for $|z| \leq 1$, n sufficiently large and $k<n$ we have 


$$
\left|g_{k, n}(z)\right| \leq \frac{2 \mu}{n}\left\{\left|1-Q\left(\alpha_{n}^{k}\right)\right|+\left|Q\left(\alpha_{n}^{k} z\right)-Q(z)\right|+\frac{2 \mu}{n}\right\} .
$$

Since for $|z| \leq 1$ and $k<n$

$$
\begin{aligned}
\left|Q\left(\alpha_{n}^{k} z\right)-Q(z)\right| & =\left|\sum_{m=0}^{\infty} q_{m}\left(\alpha_{n}^{k m}-1\right) z^{m}\right| \leq \sum_{m=0}^{\infty} q_{m}\left(1-\alpha_{n}^{k m}\right)= \\
& =1-Q\left(\alpha_{n}^{k}\right) \leq 1-Q\left(\alpha_{n}^{n}\right)
\end{aligned}
$$

we obtain an upperbound for $\left|g_{k_{n}, n}(z)\right|$ that is independent of $k$ and $z$ :

$$
\left|g_{k, n}(z)\right| \leq \frac{4 \mu}{n}\left\{1-Q\left(\alpha_{n}^{n}\right)+\frac{\mu}{n}\right\}
$$

Finally, from $\lim _{z \uparrow 1} Q(z)=1$ and $\lim _{n \rightarrow \infty} \alpha_{n}^{n}=\lim _{n \rightarrow \infty}\left(1-n^{-2}\right)^{n}=1$ it follows that $\lim _{n \rightarrow \infty} Q\left(\alpha_{n}^{n}\right)=1$, and so, for $n$ sufficiently large,

$$
(2.4 .10) \quad \forall \forall_{k \in\{0,1, \ldots, n\}} \forall_{|z| \leq 1}\left|g_{k, n}(z)\right| \leq \varepsilon_{n} \text {, with } \varepsilon_{n}=0\left(\frac{1}{n}\right)(n \rightarrow \infty) .
$$

Now, observing that for $\mathrm{n}$ sufficiently large

$$
\begin{aligned}
\left|\log \left\{1+g_{k, n}(z)\right\}\right| & \leq-\log \left\{1-\left|g_{k, n}(z)\right|\right\} \leq \\
& \leq\left|g_{k, n}(z)\right| /\left\{1-\left|g_{k, n}(z)\right|\right\} \leq 2\left|g_{k, n}(z)\right| .
\end{aligned}
$$

we can estimate as follows:

$$
\begin{aligned}
\left|\log \prod_{k=0}^{n-1}\left\{1+g_{k, n}(z)\right\}\right| & \leq \sum_{k=0}^{n-1}\left|\log \left\{1+g_{k, n}(z)\right\}\right| \leq 2 \sum_{k=0}^{n-1}\left|g_{k, n}(z)\right| \leq \\
& \leq 2 n \varepsilon_{n^{\prime}}
\end{aligned}
$$

which by $(2.4 .10)$ becomes arbitrarily small for $n \rightarrow \infty$. Hence

$$
\lim _{n \rightarrow \infty} \prod_{k=0}^{n-1}\left\{1+g_{k, n}(z)\right\}=1,
$$

and $(2.4 .7)$ immediately follows from $(2.4 .8)$ and $(2.4 .9)$.

Before turning to some examples, we mention a characterization of $C_{\alpha}$ in terms of $\mathrm{C}_{0}$.

THEOREM 2.4.5. Let $P$ be a pgf, with $P(0)>0$, let $0<\alpha<1$ and let $P_{\alpha}$ be defined by 
(2.4.11) $\quad P_{\alpha}(z):=P(\alpha) P(z) / P(\alpha z)$.

Then $P \in C_{\alpha}$ iff the function $P_{\alpha}$ is a pgf in $C_{0}$.

PROOF. First we note that if $P \in C_{\alpha}$ then by lema $2.3 .6 P_{\alpha}$ is a pgf. Next, consider the case that $\mathrm{P}_{\alpha}$ is a pgf. Then the $\mathrm{R}_{\mathrm{O}}$-function of $\mathrm{P}_{\alpha}$ is given by

$$
R_{0}^{(\alpha)}(z)=\frac{1}{z}\left\{1-P_{\alpha}(0) / P_{\alpha}(z)\right\}=\left(1-\alpha_{0}\right) R_{\alpha}(z) .
$$

Hence $R_{0}^{(\alpha)}$ is abs mon iff $R_{\alpha}$ is abs mon, from which the theorem follows.

Using theorem 2.3 .7 we can now improve theorem 2.4 .2 (iii) in the following way.

COROLLARY 2.4.6. If $P \in C_{\alpha}$ and if $0 \leq \gamma \leq 1$, then $P_{\gamma}$, defined by (2.4.11), is a pgf, while $P_{\gamma} \in C_{\alpha}$ for $0 \leq \gamma<\alpha$, and $P_{\gamma} \in C_{0}$ for $\alpha \leq \gamma \leq 1$.

Theorem 2.4 .5 is not only convenient to prove properties of the $C_{\alpha}{ }^{8} s, e . g$. the properties in theorem 2.4.2(i), (ii) and (iv), but it also provides examples of distributions in $C_{\alpha_{b}}$. This will be clear from the following obvious reformulation.

COROLIARY 2.4.7. Let $Q$ be a pgf with $Q(0)>0$, and let $0<\alpha<1$. Then $Q \in C_{0}$ iff $P(z):=\prod_{k=0}^{\infty} Q\left(\alpha^{k} z\right) / Q\left(\alpha^{k}\right)$ is a pgf in $C_{\alpha}$.

Combining corollaries 2.4 .3 and 2.4 .7 , we get for all $\alpha \in(0,1)$

$$
\text { (2.4.12) } Q \in \mathcal{C}_{0} \Rightarrow \prod_{k=0}^{n-1} Q\left(\alpha^{k} z\right) / Q\left(\alpha^{k}\right) \in C_{\alpha} \quad(n \in \mathbb{N} \cup\{\infty\}) .
$$

This will be used presently to construct examples of distributions in $C_{\alpha}$. Note that on account of theorem 1.5.13 for $Q$ we may take the pgf of any comp mon or log-convex distribution.

Now, we list a number of examples of (inf div) lattice distributions in the various classes $C_{\alpha}$. If the proofs of the statements made are straightforward, we only give brief indications. 
1. Consider the Poisson distribution with parameter $\mu>0$

$$
p_{n}=\frac{\mu^{n}}{n !} e^{-\mu} \quad\left(n \in \mathbb{N}_{0}\right) \text {, or } P(z)=\exp [\mu(z-1)]
$$

Calculating the $r_{n}(\alpha)^{\prime}$ 's corresponding to $\left\{p_{n}\right\}$ we see that

$(2.4 .13) \quad \exp [\mu(z-1)] \notin \bigcup_{0 \leq \alpha<1}^{U} C_{\alpha} \quad(\mu>0)$.

2. The negative-binomial distribution with parameters $p \in[0,1)$ and $u>0$ is defined by

$(2.4 .14) \quad p_{n}=\left(\begin{array}{c}n+u-1 \\ n\end{array}\right)(1-p) u^{u} \quad\left(n \in \mathbb{N}_{0}\right)$, or $P(z)=\left(\frac{1-p}{1-p z}\right)^{u}$.

For $u=1 \quad\left\{p_{n}\right\}$ is comp mon, hence by theorem 2.4.2(ii) we have

(2.4.15) $\quad\left(\frac{1-p}{1-p z}\right)^{u} \in C_{0} \quad(0<u \leq 1 ; 0 \leq p<1)$.

However, if u exceeds 1 , the classes $C_{\alpha}(0<\alpha<1)$ are skipped over:

(2.4.16) $\quad\left(\frac{1-p}{1-p z}\right)^{u} \& \underset{0 \leq \alpha<1}{U} C_{\alpha} \quad(u>1 ; 0 \leq p<1)$.

To prove this, we put $u=1+\varepsilon$ with $\varepsilon>0$ and obtain for the $R_{\alpha}$-function of $P$

$$
(1-\alpha) z R_{\alpha}(z)=1-\left(\frac{1-p z}{1-p \alpha z}\right)^{1+\varepsilon}=1-\left\{1-\frac{p(1-\alpha) z}{1-p \alpha z}\right\}^{1+\varepsilon} .
$$

Expanding this in a power series in $z$ and putting $x=(1-\alpha) / \alpha$, we see that for $n \in \mathbb{N}$

$$
\begin{aligned}
(1-\alpha)(p \alpha)^{-n_{x_{n-1}}(\alpha)} & =(-1)^{n-1} \sum_{k=1}^{n}\left(\begin{array}{c}
1+\varepsilon \\
k
\end{array}\right)\left(\begin{array}{l}
-k \\
n-k
\end{array}\right) x^{k}= \\
& =\sum_{k=1}^{n}(-1)^{k-1}\left(\begin{array}{c}
1+\varepsilon \\
k
\end{array}\right)\left(\begin{array}{c}
n-1 \\
k-1
\end{array}\right) x^{k} .
\end{aligned}
$$

For the case $0<\varepsilon \leq 1$ it follows that

$$
\begin{aligned}
& (1-\alpha)(p \alpha)^{-n_{r}} r_{n-1}(\alpha)=(1+\varepsilon) x-(1+\varepsilon) \varepsilon \sum_{k=2}^{n} \frac{1}{k(k-1)}\left(\begin{array}{c}
k-2-\varepsilon \\
k-2
\end{array}\right)\left(\begin{array}{c}
n-1 \\
k-1
\end{array}\right) x^{k} \leq \\
& \leq(1+\varepsilon) x-\frac{1}{2}(n-1)(1+\varepsilon) \varepsilon x^{2} .
\end{aligned}
$$

which tends to $-\infty$ for $n \rightarrow \infty$. Hence $(2.4 .16)$ is proved for $1<u \leq 2$. 
Next, suppose $u>2$ and that $\{(1-p) /(1-p z)\}^{u} \in \mathcal{C}_{\alpha}$ for some $\alpha \in[0,1)$. Then according to theorem 2.4.2(ii) it would follow that for all $v \in(1,2]$

$$
\left(\frac{1-p}{1-p z}\right)^{v}=\left\{\left(\frac{1-p}{1-p z}\right)^{u}\right\}^{v / u} \in C_{\alpha}
$$

but this contradicts the result above.

3. Consider the boundary distribution $\left\{\tilde{p}_{n}\right\}$ with parameters $\alpha_{0} \in[0,1]$ and $\gamma \in\left(0,\left(1-a_{0}\right)^{-1}\right)$ (cf. definition 2.2.2); it satisfies

(2.4.17) $\quad \tilde{p}_{n}=\tilde{p}_{0} \gamma^{n} \prod_{k=0}^{n-1} c_{k}\left(\alpha_{0}\right)^{-1} \quad(n \in \mathbb{N})$.

For $\alpha_{0}=0$ we get the geometric distribution with parameter $\gamma_{0}$ and for $\alpha_{0}=1$ the poisson distribution with parameter $\gamma$, which has been considered in example 1. Now, take $\alpha_{0} \in(0,1)$. By $(2.2 .2)$ and $(2.2 .3)$ we know that $\left\{\tilde{p}_{n}\right\} \in \mathcal{C}_{\alpha_{0}}$ with $R_{\alpha_{0}} \equiv \gamma$. Hence by $(2.3 .7)$ we have for the pgf $\widetilde{\mathrm{p}}$ of $\left\{\tilde{\mathrm{p}}_{\mathrm{n}}\right\}$

(2.4.18) $\tilde{\mathrm{p}}(z)=\prod_{k=0}^{\infty} \frac{1-\left(1-\alpha_{0}\right) \gamma \alpha_{0}^{k}}{1-\left(1-\alpha_{0}\right) \gamma \alpha_{0}^{k}} \quad(|z| \leq 1)$.

In order to check whether $\widetilde{\mathrm{P}} \in \mathcal{C}_{\alpha}$ for some $\alpha<\alpha_{0}$, we calculate

$$
(1-\alpha) z R_{\alpha}(z)=1-\prod_{k=0}^{\infty} \frac{1-\left(1-\alpha_{0}\right) \gamma \alpha_{0}^{k} z}{1-\left(1-\alpha_{0}\right) \gamma \alpha_{0}^{k} \alpha z},
$$

from which it follows that for $m \in \mathbb{N}$

$$
\left(1-\alpha_{0}^{\mathrm{m}}\right) \mathrm{zR}_{\alpha_{0}}(z)=1-\prod_{\mathrm{k}=0}^{\mathrm{m}=1}\left\{1-\left(1-\alpha_{0}\right) \gamma \alpha_{0}^{k} z\right\} .
$$

As this is a polynomial in $z$ of degree $m$, we must have $r_{n}\left(\alpha_{0}^{m}\right)=0$ for $\mathrm{n}=\mathrm{m}, \mathrm{m}+1, \ldots$ or, equivalently,

$$
\forall_{n \in \mathbb{N}}{ }_{m \in\{1, \ldots, n\}} r_{n}\left(\alpha_{0}^{m}\right)=0
$$

From (2.4.17) and the recurrence relations (2.3.1), however, we see that $x_{n}(\alpha)$ is a polynomial in $\alpha$ of degree $n$ with leading coefficient $\gamma^{n+1} \prod_{k=0}^{n} c_{k}\left(\alpha_{0}\right)^{-1}$, so that 
(2.4.19) $r_{n}(\alpha)=\gamma^{n+1} \prod_{k=1}^{n} \frac{\alpha-\alpha_{0}^{k}}{c_{k}\left(\alpha_{0}\right)} \quad\left(n \in \mathbb{N}_{0} ; 0 \leq \alpha<1\right)$.

From this we obtain (take $\mathrm{n}=1$ ) for every choice of the parameters $\alpha_{0}$ and $\gamma$ $(2.4 .20) \quad\left\{\tilde{p}_{n}\right\} \in \mathcal{C}_{\alpha} \Leftrightarrow \alpha \geq \alpha_{0}$.

4. The Logarithmic distribution with parameter $\theta \in(0,1)$, given by

$$
(2.4 .21) \quad p_{n}=\frac{1}{b} \frac{1}{n+1} \theta^{n+1} \quad\left(n \in \mathbb{N}_{0}\right) \text {, or } P(z)=-\frac{1}{b z} \log (1-\theta z),
$$

where $b:=-\log (1-\theta)$, is comp mon, and hence in $C_{0}$. The following related distribution, which will be called the semi-Zogarithmic distribution with parameters $a>0$ and $\theta \in(0,1)$, is more interesting:

$$
(2.4 .22) \quad p_{0}=\frac{a}{1+a}, p_{n}=\frac{1}{(1+a) b} \frac{1}{n} \theta^{n} \quad(n \in \mathbb{N})
$$

where again $b:=-\log (1-\theta)$. Its pgf $P$ is given by

$(2.4 .23) \quad P(z)=\frac{a}{1+a}\left\{1-\frac{1}{a b} \log (1-\theta z)\right\}$.

This distribution is also considered by Katti (1967), who proves that $\left\{p_{n}\right\}$ is inf div iff $a b \geq 1$, and by steutel (1970), who notes that $\left\{p_{n}\right\}$ is $\log$ convex (and so in $C_{0}$ ) if $a b \geq 2$. For the case $1 \leq a b \leq 2$ we now prove the following result:

$$
(2.4 .24) \quad\left\{p_{n}\right\} \in C_{\alpha} \Leftrightarrow a b \geq \frac{2}{1+\alpha} \quad(0 \leq \alpha \leq 1) .
$$

To this end we consider the recurrence relations (2.3.1) rather than the re-lation (2.3.2) for $\mathrm{pgf}^{3} \mathrm{~s}$, and obtain

$$
c_{n}(\alpha) \frac{\theta^{n+1}}{n+1}=a b x_{n}(\alpha)+\sum_{k=1}^{n} \frac{\theta^{k}}{k} x_{n-k}(\alpha) \quad\left(n \in \mathbb{I N}_{0}\right)
$$

or, defining $\bar{x}_{n}(\alpha):=\theta^{-n-1} r_{n}(\alpha)$,

$$
(2.4 .25) \quad 1=\frac{n+1}{c_{n}(\alpha)}\left\{a b \bar{x}_{n}(\alpha)+\sum_{k=1}^{n} \frac{1}{k} \bar{x}_{n-k}(\alpha)\right\} \quad\left(n \in \mathbb{N}_{0}\right) .
$$

For $\mathrm{n}=1$ we get $a b \bar{r}_{1}(\alpha)=\frac{1}{2}(1+\alpha)-(a b)^{-1}$; hence the condition in the right-hand side of $(2.4 .24)$ is necessary. To prove its sufficiency, from $(2.4 .25)$ we subtract the same relation with $n$ replaced by $n-1$, and obtain 


$$
\begin{aligned}
a b \bar{r}_{n}(\alpha) & =\sum_{k=2}^{n}\left[\frac{1}{k-1} \frac{n}{n+1} \frac{c_{n}(\alpha)}{c_{n-1}(\alpha)}-\frac{1}{k}\right] \bar{r}_{n-k}(\alpha)+ \\
& +\left[\frac{n}{n+1} \frac{c_{n}(\alpha)}{c_{n-1}(\alpha)} a b-1\right] \bar{r}_{n-1}(\alpha) .
\end{aligned}
$$

By the monotonicity of $c_{n}(\alpha)$ in $n$ the coefficients of the $\bar{x}_{n-k}(\alpha)$ 's are all positive; therefore it is sufficient to prove that under the condition in $(2.4 .24)$ the following holds:

$$
a b \geq \frac{n+1}{n} \frac{1-\alpha^{n}}{1-\alpha^{n+1}}=: a_{n}(\alpha) \quad(n \in \mathbb{N} ; 0<\alpha<1) .
$$

This immediately follows from the fact that $d_{1}(\alpha)=2 /(1+\alpha)$ and that $\mathrm{d}_{\mathrm{n}}(\alpha) \leq \mathrm{d}_{1}(\alpha)$ for all $\mathrm{n} \in \mathbb{I N}$ and all $\alpha \in(0,1)$. The last assertion is easily verified by noting that for the function $f$, defined on $(0,1)$ by $f(\alpha):=$ $2\left(1-\alpha^{n+1}\right) /(n+1)-(1+\alpha)\left(1-\alpha^{n}\right) / n$, one has $f(0)>0, f(1)=0, f^{3} \leq 0$ on $(0,1)$.

5. A product of two geometric pgf's can be written as

$$
(2.4 .26) \quad p(z)=\frac{1-p}{1-p z} \frac{1-p \gamma}{1-p \gamma z} \text {, or } p_{n}=(1-p)(1-p \gamma) c_{n}(\gamma) p^{n} \quad\left(n \in \mathbb{N}_{0}\right) \text {. }
$$

where $0 \leq \mathrm{p}<1$ and $0 \leq \gamma \leq 1$. From the $\mathrm{R}_{\alpha}$-function of $\mathrm{P}$ we find

$$
r_{0}(\alpha)=(1+\gamma) p, r_{n}(\alpha)=\left\{c_{n+1}(\gamma) \alpha-\gamma c_{n-1}(\gamma)\right\}_{p}^{n+1} \alpha^{n-1} \quad(n \in \mathbb{N}) .
$$

Now, the zero $\alpha_{n}:=\gamma c_{n-1}(\gamma) / c_{n+1}(\gamma)$ of $x_{n}(\alpha)$ is nondecreasing in $n$ and $\lim _{n \rightarrow \infty} \alpha_{n}=\gamma$, from which it follows that

$(2.4 .27) \quad \frac{1-p}{1-p z} \frac{1-p \gamma}{1-p \gamma z} \in C_{\alpha} \Leftrightarrow \alpha \geq \gamma \quad(0 \leq p<1 ; 0 \leq \gamma \leq 1)$.

6. For a product of three geometric pgf's we find in a similar way

(2.4.28) $\quad \frac{1-p}{1-p z} \frac{1-p \gamma}{1-p \gamma z} \frac{1-p \gamma \delta}{1-p \gamma \delta z} \in C_{\max (\gamma, \delta)} \quad(0 \leq p<1 ; 0 \leq \gamma \leq 1 ; 0 \leq \delta \leq 1)$.

The following examples are special cases of (2.4.12).

7. $\prod_{k=0}^{n-1} \frac{1-p \alpha^{k}}{1-p \alpha^{k}{ }^{k}} \in C_{\alpha} \quad(0 \leq p<1 ; n \in \mathbb{N} \cup\{\infty\})$.

Note that for $\mathrm{n}=\infty$ we get the boundary distribution from example 3 . 
8. $\prod_{k=0}^{n-1} \frac{\gamma-\log \left(1-\theta \alpha^{k} z\right)}{\gamma-\log \left(1-\theta \alpha^{k}\right)} \in C_{\alpha} \quad(0<\theta<1 ; \gamma \geq 2 ; n \in \mathbb{N} \cup\{\infty\})$.

9. Lamperti (1958) notes that $\tan z /(z \tan 1)$ is a pgf in $C_{0}$. Hence

$$
\prod_{k=0}^{n-1} \frac{\tan \left(\alpha^{k} z\right)}{z \tan \alpha^{k}} \in \mathcal{C}_{\alpha}
$$

10. It is easy to see that the quotient of two geometric pgf's with parameters $\mathrm{p}$ and $\mathrm{q}$, respectively, is again a pgf iff $\mathrm{p} \geq \mathrm{q}$. In that case it belongs necessarily to $C_{0}$. Hence we have

$$
\prod_{k=0}^{n-1} \frac{1-p \alpha^{k}}{1-p \alpha^{k} z} / \frac{1-p \gamma \alpha^{k}}{1-p \gamma \alpha^{k}} \in C_{\alpha} \quad(0 \leq p<1 ; 0 \leq \gamma \leq 1 ; n \in \mathbb{N} \cup\{\infty\}) .
$$

The characterization of $C_{\alpha}$ by $C_{0}$, given in theorem 2.4 .5 , only holds for $\alpha<1$. In the case $\alpha=1$, however, there also exists such a characterization; in fact, we have the following analogue of theorem 2.4 .5 and corollary 2.4 .7 .

THEOREM 2.4.8. Let $P$ be a pgf with $P(0)>0$. Then (i) $P \in C_{1}$ iff $\{1-\log P(z)\}^{-1}$ is a pgf in $C_{0}$, (ii) $P \in C_{0}$ iff $\exp \left[1-P(z)^{-1}\right]$ is a pgf in $C_{1}$.

PRCOF. The theorem immediately follows from the following two observations. If $P$ is compound-Poisson- $(\mu, Q)$, then

$$
\{1-\log P(z)\}^{-1}=\{1-\mu(Q(z)-1)\}^{-1}=\{1+\mu-\mu Q(z)\}^{-1},
$$

which is compound-geometric- $(p, Q)$ with $p=\mu /(1+\mu)$.

Conversely, if $P$ is compound-geometric- $(p, Q)$, then

$$
\exp \left[1-P(z)^{-1}\right]=\exp [1-(1-p Q(z)) /(1-p)] .
$$

which is compound-Poisson- $(\mu, 2)$ with $\mu=p /(1-p)$.

Using simple properties of $C_{1}$, from the preceding theorem we obtain some operations, under which $C_{0}$ is closed. Closure under operations on the $p_{n}$ 's themselves will be discussed in section 4.1 . 
THEOREM 2.4 .9 .

(i) If $P \in C_{0}$ and $\mu>0$, then $\mu /\{\mu-\log P(z)\} \in \mathcal{C}_{0^{*}}$

(ii) If $P \in C_{0}$ and $\gamma>0$, then $P(z) /\{\gamma+(1-\gamma) P(z)\} \in C_{0}$.

(iii) If $\mathrm{P} \in \mathrm{C}_{0}$ and $0 \leq \mathrm{p} \leq 1$, then $\mathrm{p}+(1-\mathrm{p}) \mathrm{P}(z) \in \mathcal{C}_{0}$.

(iv) If $\mathbb{P}_{1} \in C_{0}$ and $P_{2} \in C_{0}$, then $P_{1}(z) P_{2}(z) /\left\{P_{1}(z)+P_{2}(z)-P_{1}(z) P_{2}(z)\right\} \in C_{0}$.

PROOF。

(i) If $\mathrm{P} \in \mathrm{C}_{0}$, then by theorem 1.4 .4 we have $\mathrm{P}^{1 / \mu} \in \mathrm{C}_{1}$ for all $\mu>0$. The result follows by application of theorem $2.4 .8(i)$.

(ii) Using theorem 2.4 .8 , we see that if $P \in C_{0}$, then $Q(z):=\exp \left[1-P(z)^{-1}\right]$, and hence for all $\gamma>02^{\gamma}$, is a pgf in $C_{1}$. It follows that

$$
\left\{1-\log Q(z)^{\gamma}\right\}^{-1}=\left\{1-\gamma\left(1-P(z)^{-1}\right)\right\}^{-1}=\frac{P(z)}{\gamma+(1-\gamma) P(z)}
$$

is a pgfin $C_{0}$.

(iii) Let $P \in \mathcal{C}_{0}$ and $0 \leq p \leq 1$. Then for the $R_{0}$-function $R_{0}^{(p)}$ of $p+(1-p) P$ we have

$$
z R_{0}^{(p)}(z)=1-\frac{p+(1-p) P(0)}{p+(1-p) P(z)}=(1-p) \frac{P(z)}{p+(1-p) P(z)} z R_{0}(z)
$$

which is abs mon because of (ii). Hence $p+(1-p) P \in C_{0}$.

(iv) If $P_{j} \in C_{0}(j=1,2)$, then also $Q_{j}(z):=\exp \left[1-P_{j}(z)^{-1}\right] \in C_{1}(j=1,2)$. It follows that $Q_{1} Q_{2} \in C_{1}$, and hence the following function is a pgf in $C_{0}$ :

$$
\begin{aligned}
& \left\{1-\log Q_{1}(z) Q_{2}(z)\right\}^{-1}=\left\{1-\left[1-P_{1}(z)^{-1}\right]-\left[1-P_{2}(z)^{-1}\right]\right\}^{-1}= \\
& =P_{1}(z) P_{2}(z) /\left\{P_{1}(z)+P_{2}(z)-P_{1}(z) P_{2}(z)\right\} .
\end{aligned}
$$

As in the case $\alpha<1$, it is possible, by use of theorem 2.4.8, to construct examples of inf div distributions from specially chosen distributions in $C_{0}$, and conversely. In the following theorem we mention a general class of inf div distributions, obtained in such a way.

THEOREM 2.4.10. For every df $G$ on $[0,1)$ the function $Q$, defined by

$$
(2.4 .29) \quad Q(z):=\exp \left[1-\left\{\int_{[0,1)} \frac{1-p}{1-p z} d G(p)\right\}^{-1}\right] .
$$

is a pgf in $C_{1}$. 
PROOF. According to lemma 1.5.11 the pgf of a comp mon lattice distribution $\left\{p_{n}\right\}_{0}^{\infty}$ has the form

$$
P(z)=\sum_{n=0}^{\infty} \int_{[0,1)}(1-p) p^{n} d G(p) z^{n}=\int_{[0,1)} \frac{1-p}{1-p z} d G(p),
$$

where $G$ is a df on $[0,1)$. As by theorem $1.5 .13 \mathrm{P} \in C_{0}$, the theorem follows from theorem 2.4 .8 (ii).

Next we list a few examples, which are all obtained by using theorem 2.4.8.

1. Considering the semi-logarithmic distribution (cf. $(2.4 .22)$ ), we get

$$
\exp \left[\frac{-b-\log (1-\theta z)}{a b-\log (1-\theta z)}\right] \in C_{1} \quad \text { iff } a b \geq 2 \text {, }
$$

where $\mathrm{a}>0,0<\theta<1$ and $\mathrm{b}:=-\log (1-\theta)$.

2. As noted in example 9, p. 60, $\tan z /(z \tan 1)$ is a pgf in $C_{0}$. Hence

$$
\exp [1-z \tan 1 / \tan z] \in \mathcal{C}_{1} \text {. }
$$

3. Taking the semi-logarithmic distribution with $a b=1$, we get

$$
\left\{1-\log \frac{1-\log (1-\theta z)}{1-\log (1-\theta)}\right\}^{-1} \in C_{0} .
$$

4. As $\frac{1+p z}{1+p} \frac{1-p}{1-p z}$ is a pgf in $C_{1}$ (and not in $C_{\alpha}$ for $\alpha<1$ ), we have

$$
\left\{1-\log \frac{1-p}{1+p} \frac{1+p z}{1-p z}\right\}^{-1} \in C_{0} .
$$

Finally, we mention a property of $C_{0}$ that ensures the product of a geometric pgf and a pgf from a large subclass of $C_{0}$ to be again in $C_{0}$. Note, that in general the product of two $C_{0}$-pgf's does not belong to $C_{0}$ (cf. $(2.4 .27)$ ). This property is suggested by replacing the exponential distribution by its discrete analogue, the geometric distribution, in a similar property of the analogue of $C_{0}$ for distributions on $[0, \infty)$, the class $F_{\infty}$ (cf. theorem 5.4.19). THEOREM 2.4.11. If $p_{,} p_{1}$ and $p_{2} \in[0,1)$ and if $Q$ is a pgf, then (2.4.30) $\quad P(z):=\frac{1-p_{1}}{1-p_{1} z} \frac{1-p}{1-p\left(\left(1-p_{2}\right) /\left(1-p_{2} z\right)\right\} Q(z)} \in C_{0} \quad$ if $p_{1} \leq p_{2}$. 
PROOF. It is no restriction to suppose that $Q(0)=0$, as a pgf $P$ of the form $(2.4 .30)$ can be written in the same form with different $p$ and $Q$, such that $Q(0)=0$. For the $R_{0}$-function of $P$ we can write now

$$
\begin{aligned}
\mathrm{zR}_{0}(z) & =1-\frac{\mathrm{P}(0)}{\mathrm{P}(\mathrm{z})}=1-\left(1-\mathrm{p}_{1} z\right)\left\{1-\mathrm{p} \frac{1-\mathrm{p}_{2}}{1-\mathrm{p}_{2} z} \mathrm{Q}(\mathrm{z})\right\}= \\
& =p_{1} z+p\left(1-\mathrm{p}_{2}\right) \frac{1-\mathrm{p}_{1} z}{1-\mathrm{p}_{2} z} \mathrm{Q}(\mathrm{z})= \\
& =p_{1} z+p\left(1-p_{2}\right) Q(z)+p\left(p_{2}-p_{1}\right) z \frac{1-p_{2}}{1-p_{2} z} Q(z)
\end{aligned}
$$

which is an abs mon function, if $p_{1} \leq p_{2}$. Hence $P \in C_{0}$.

\subsection{Other classifications}

The classes $C_{\alpha}$, introduced by means of recurrence relations, were seen to consist of infinite products of certain compound geometric pgf's. We will now start from classes of similar pgf's, the classes $C_{0}^{\mathrm{u}}$ of the compound negative-binomial pgf's with parameter $u \geq 0$ (cf. (2.4.14)). Also these classes define a classification of $C_{1}$, but there is no characterization by means of recurrence relations of type $(2.1 .4)$.

DEFINITION 2.5.1. For $u \geq 0$ a lattice distribution $\left\{p_{n}\right\}_{0}^{\infty}$, with $p_{0}>0$, is said to be in the class $C_{0}^{\mathrm{u}}$ if its pgf $\mathrm{P}$ has the form

$(2.5 .1) \quad P(z)=\left\{\frac{1-p}{1-p Q(z)}\right\}^{u}$

where $0 \leq p<1$ and $Q$ is a pgf with $Q(0)=0$.

clearly, $C_{0}^{0}$ only consists of the degenerate distribution at zero, $C_{0}^{1}=C_{0}$ and $C_{o}^{u} \subset C_{1}$ for all $u \geq 0$. The following obvious criterion is useful.

LEMMA 2.5.2. Let $P$ be a pgf with $P(0)>0$, and let $u>0$. Then $P \in C_{0}^{u}$ iff $p^{1 / u}$ is a pge in $C_{0}$.

It is now easily shown that the classes $C_{0}^{u}$ define a classification of the inf div lattice distributions. 
THEOREM 2.5.3. $\mathrm{C}_{0}^{\mathrm{u}}$ is nondecreasing in $u \in[0, \infty)$, i.e. for all $u$ and $v \in[0, \infty)$

$$
C_{0}^{u}<C_{0}^{v} \text { if } u<v
$$

PROOF. Let $0<u<v$ and $P \in C_{0}^{u}$. Then $P^{1 / u}$ is a pgf in $C_{0}$ so that according to theorem $2.4 .2(i i)$ also $\mathrm{P}^{1 / \mathrm{v}}=\left(\mathrm{P}^{1 / \mathrm{u}}\right)^{\mathrm{u} / \mathrm{v}}$ is a pgf in $\mathrm{C}_{0}$. Hence $\mathrm{P} \in \mathrm{C}_{0}^{\mathrm{V}} \cdot \mathrm{D}$

In fact, interpolation between $(1-p) /(1-p Q(z)) \in C_{0}$ and $\exp [\mu(Q(z)-1)] \in C_{1}$ is most easily achieved by considering pgf's of the form

$$
\mathrm{P}_{u}(z):=\left\{\frac{1-\mu / u}{1-(\mu / u) Q(z)}\right\}^{u} \quad(u>\mu) .
$$

Using these pgf's we easily see that, like $\underset{0 \leq \alpha<1}{U} C_{\alpha}{ }_{u>0}^{U} C_{0}^{u}$ is dense in $C_{1}$. We state this in the following theorem.

THEOREM 2.5.4. If $\mathrm{P} \in \mathrm{C}_{1}$, then there exists a sequence of pgf's $\mathrm{P}_{n} \in \mathrm{C}_{0}^{n}$ ( $n \in \mathbb{N}$ ) such that $P(z)=\lim _{n \rightarrow \infty} P_{n}(z)$ for $|z| \leq 1$.

Although little can be said about the relation between the $C_{0}^{u} s$ and the $C_{\alpha}{ }^{3} s(\mathrm{cf} .(2.4 .16))$, the $C_{0}^{u^{\prime}} \mathrm{s}$ possess the same sort of properties the $C_{\alpha}{ }^{\circ} s$ have. In this respect the functions $\mathrm{p}^{1 / u}$ for $C_{0}^{u}$, and $P(z) / P(\alpha z)$ for $C_{\alpha}$, play analogous roles. The following properties of $c_{0}^{\mathrm{u}}$ are very similar to those of $C_{\alpha}$ (Cf. theorem 2.4.2), and are easily verified by using lemma 2.5 .2 and theorem 2.4 .2 with $\alpha=0$.

THEOREM 2.5.5. Let $u>0$.

(i) If a pgf $P$ is the limit of a sequence of pgf's $P_{n} \in C_{0}^{U}$, then also $p \in C_{0}^{u}$

(ii) If $P \in C_{0}^{U}$ and $0 \leq \gamma \leq 1$, then $P(\gamma z) / P(\gamma) \in C_{0}^{U}$.

(iii) If $\mathrm{P} \in \mathrm{C}_{0}^{\mathrm{u}}$ and $\gamma \geq 0$, then $\mathrm{P}^{\gamma} \in \mathrm{C}_{0}^{\gamma \mathrm{u}}$.

(iv) If $P \in C_{0}^{U}$ and $0 \leq \gamma \leq 1$, then $P(\gamma) P(z) / P(\gamma z) \in C_{0}^{U}$.

Next, we briefly consider classes of gf's that turn out to be closely related to the classes $C_{0}^{u}$ " They are suggested by the characterization of $C_{1}$ by means of the abs mon of $R_{1}(z)=p^{\prime}(z) / P(z)$ (cf. theorem 1.5.3) and the following characterization of $\mathrm{C}_{0}$.

LEMMA 2.5.6. A pgf $P$, with $P(0)>0$, is in $C_{0}$ iff $-\frac{d}{d z} P(z)^{-1}=P^{3}(z) / P(z)^{2}$ is an abs mon function. 
PROOF. According to theorem 1.5.8 $\mathrm{p}$ is in $C_{0}$ iff $R_{0}(z)=z^{-1}\{1-P(0) / P(z)\}$ is abs mon. Clearly, this is equivalent to the abs mon of $\frac{d}{d z}\left[z R_{0}(z)\right]$, and the lemma is proved.

DEFINITION 2.5.7. For $\gamma \in \mathbb{R}$ a gf $P$, with $P(0)>0$ and $P(1)=1$, is said to be in the class $H_{\gamma}$ if the function $S_{\gamma}$, defined by

$(2.5 .2) \quad S_{\gamma}(z):=P^{v}(z) / P(z)^{1+\gamma}$,

is abs mon.

The function $S_{\gamma}$ in $(2.5 .2)$ can be written in the following form:

(2.5.3) $\quad S_{\gamma}(z)= \begin{cases}\frac{d}{d z} \log P(z), & \text { if } \gamma=0 \\ -\frac{1}{\gamma} \frac{d}{d z} P(z)^{-\gamma}, & \text { if } \gamma \neq 0 .\end{cases}$

Although we do not require the coefficients of a gf $\mathrm{P} \in H_{\gamma}$ to be nonnegative, the classes $H_{\gamma}$, with $\gamma \geq 0$, turn out to contain only pgf's.

Considexing first the case $\gamma=0$, we solve (2.5.3) for $P$ and obtain

$(2.5 .4) \quad P(z)=P(0) \exp \left[\int_{(0, z]} s_{0}(u) d u\right]$.

It follows that if $P \in H_{0}$ then by lemma $1.3 .3(v) P$ is a pgf. Hence, as $S_{0}=R_{1}$, we conclude that

$(2.5 .5) \quad H_{0}=C_{1}$.

Denoting the coefficients in the power-series expansion of $S_{\gamma}$ by $s_{n}(\gamma)$ $\left(n \in \mathbb{N}_{0}\right.$ ), we obtain from (2.5.2) for all $\gamma \in \mathbb{R}$ a characterization of $H_{\gamma}$ by means of relations that, like $(2.1 .4)$, generalize the known recurrence relations (2.1.1) for $C_{1}$, but are much less attractive than (2.1.4).

LEMMA 2.5.8. For $\gamma \in \mathbb{R}$ a sequence $\left\{p_{n}\right\}^{\infty}$, with $p_{0}>0$ and $\sum p_{n}=1$, is in $H_{\gamma}$ iff there exist $s_{n}(\gamma) \geq 0\left(n \in \mathbb{N}_{0}\right)$ such that

$(2.5 .6) \quad(n+1) p_{n+1}=\sum_{k=0}^{n} p_{k}^{*(1+\gamma)} s_{n-k}(\gamma) \quad\left(n \in \mathbb{N}_{0}\right)$ 
The case $\gamma<0$ is not very interesting, as is apparent from the following properties, which we give without their, simple, proofs.

LEMMA 2.5.9. If $\gamma<0$, then a gf $P$, with $P(0)>0$ and $P(1)=1$, is in $H_{\gamma}$ iff the function $p|\gamma|$ is abs mon.

LEMMA 2.5 .10 .

(i) For all $\gamma<0: C_{1}=H_{0} \subset H_{\gamma}$.

(ii) For $n \in \mathbb{N}: H_{-1 / n}=\{$ pgf $P \mid P(0)>0$ and $P$ is $n-d i v\}$.

(iii) For $n \in \mathbb{N}: H_{-n}^{-n}=\left\{g f p \mid P(0)>0\right.$ and $p^{n}$ is a pgf $\}$.

The case $\gamma>0$ is more interesting, because $\left(H_{\gamma} \mid \gamma \geq 0\right)$ turns out to define a classification of $C_{1}$.

THEOREM 2.5.11. For $\gamma>0$ the following relation holds:

$$
H_{\gamma}=C_{o}^{1 / \gamma}
$$

In fact, if $\gamma>0$ then a gf $P$, with $P(0)>0$ and $P(1)=1$, is in $H_{\gamma}$ iff $P^{\gamma}$ is a pgf in $\mathrm{C}_{0}$.

PROOF. Let $\gamma>0$, and let $P$ be a gf with $P(0)>0$ and $P(1)=1$.

First we show that if $\mathrm{P} \in H_{\gamma}$ then $\mathrm{p}^{\gamma}$ is a pgf. So, let $\mathrm{S}_{\gamma}$ be abs mon. Solving (2.5.3) for $\mathrm{P}^{\gamma}$, we obtain

$$
\left.P(z)^{\gamma}=P(0)^{\gamma}\left\{1-\gamma P(0)^{\gamma} \int_{(0, z]} s_{\gamma}(u) d u\right)\right\}^{-1}
$$

from which by lemma 1.3.3(v) we see that $\mathrm{p}^{\gamma}$ is abs mon. Since the $s_{0}-$ function of $\mathrm{P}$ can be written as

$$
S_{0}(z)=P^{\prime}(z) / P(z)=S_{\gamma}(z) P(z)^{\gamma}
$$

it follows that $s_{\circ}$ is abs mon, and hence by $(2.5 .5)$ that $P$ is an inf div pgf. In view of theorem 1.4.4 we conclude that also $\mathrm{p}^{\gamma}$ is a $\mathrm{pgf}$.

Next, consider the case that $\mathrm{P}^{\gamma}$ is a pgf. From (2.5.3) the following relation between the $s_{1}$-function $s_{1}^{(\gamma)}$ of $p^{\gamma}$ and the $s_{\gamma}$-function $s_{\gamma}$ of $p$ is easiIy seen to hold:

$$
S_{1}^{(\gamma)}(z)=\gamma S_{\gamma}(z)
$$


from which by definition it follows that $\mathrm{P} \in H_{\gamma}$ iff $\mathrm{P}^{\gamma} \in H_{1}$. On the other hand by lemma 2.5 .6 we have $\mathrm{P}^{\gamma} \in \mathrm{C}_{0}$ iff $\mathrm{P}^{\gamma} \in H_{1}$, and hence the theorem is proved.

Thus, the $H_{\gamma}^{\prime}$ 's yield the same classification of $C_{1}$ as the $C_{0}^{u_{1}} s$. For instance, it follows that a gf $\mathrm{P}$ in $H_{\gamma}$, with $\gamma>0$, has the (canonical) representation (2.5.1) with $u=1 / \gamma$, and that the compound negative-binomial lattice distributions with parameter $u=1 / \gamma$ can be characterized by means of the relations $(2.5 .6)$. 

CHAPTER 3

DECOMPOSITIONS OF LATTICE DISTRIBUTIONS

The classes $C_{\alpha}$ from the previous chapter lead us to consider in general the pgf's $\mathrm{P}$ that have $\mathrm{P}(\alpha z) / \mathrm{P}(\alpha)$ as a factor. These pgf's, which will be called $\alpha$-decomposable ${ }^{1)}$, are briefly studied in section 1 together with the somewhat larger class of the somcalled $\alpha$-factorizable pgf's $P$, which are of the form $Q(z) Q(\alpha z) / Q(\alpha)$ with $Q$ a $p g f$.

In view of a notational analogy to the well-known self-decomposable distributions (on $\mathbb{R}$; cf. the end of section 1), also called distributions of class $\mathcal{L}$, it is then natural to consider (this is done in section 2) the classes of pgf's that are $\alpha$-decomposable and $\alpha$-factorizable, respectively, for $\alpha Z Z$

$\alpha \in(0,1)$. The former class turns out to coincide with $C_{1}$, while the latter contains $C_{1}$ as a proper subset, but nevertheless shares a number of basic properties with $C_{1}$.

In section 3 we introduce a new class of inf div lattice distributions, which is a close analogue of the class of (absolutely continuous) self-decomposable distributions on $[0, \infty)$; in fact, we consider pgf's $P$ that have $P(1-\alpha+\alpha z)$ as a factor for all $\alpha \in(0,1)$. As a subclass of this we obtain a lattice analogue of the (absolutely continuous) stable distributions.

Finally, in section 4 we briefly consider pgf"s $\mathrm{P}$ that have $1-\alpha+\alpha \mathrm{P}(z)$ as a factor. This gives rise to a new characterization of $C_{0}$.

3.1. a-decomposable and a-factorizable lattice distributions

Clearly, the characterization of $\mathrm{C}_{\alpha}$, given by theorem 2.4 .5 , can be reformulated as follows: if $P$ is a pgf with $P(0)>0$ and if $0<\alpha<1$, then

(3.1.1) $P \in C_{\alpha} \Leftrightarrow \exists_{\alpha \in C_{0}} P(z)=\frac{P(\alpha z)}{P(\alpha)} P_{\alpha}(z)$.

Dropping the condition that the factor $\mathrm{P}_{\alpha}$ be in $C_{0}$, we can generally consider pgf's $\mathrm{P}$ that have $\mathrm{P}(\alpha z) / \mathrm{P}(\alpha)$ as a factor. To this end we give the following definition.

DEFINITION 3.1.1. For $0<\alpha<1$ a pgf $\mathrm{P}$, with $\mathrm{P}(0)>0$, is said to be $\alpha-d e-$ composable $(\alpha-$ dec $)$ if there exists a pgf $P_{\alpha}$ such that

1) This concept is not related to the " $\alpha$-decompositions" considered in chapter 10 of Lukacs (1970). 
(3.1.2) $\quad P(z)=\frac{P(\alpha z)}{P(\alpha)} p_{\alpha}(z) \quad(|z| \leq 1)$.

The following property of $C_{\alpha}$, similar to (3.1.1) (cfe theorem 2.4.2(iv), with $\alpha=0$ and $n=2$ ):

(3.1.3) $Q \in C_{0} \Rightarrow P(z):=\frac{Q(\alpha z)}{Q(\alpha)} Q(z) \in C_{\alpha}$

raises the following question: is the converse of (3.1.3) also true, i.e. can every $P \in C_{\alpha}$ be represented as $Q(z) Q(\alpha z) / Q(\alpha)$ with $Q \in C_{0}$ ? This problem, which will be solved in lemma 3.1.8, lead us to consider the a-factorizable pgf's, introduced as follows.

DEFINITION 3.1.2. For $0<\alpha<1$ a pgf $P$, with $P(0)>0$, is said to be $\alpha-f a c-$ torizable $\left(\alpha\right.$-fact) if there exists a pgf ${ }^{\alpha} \mathrm{p}$ such that

(3.1.4) $\quad P(z)=\frac{\alpha_{P(\alpha z)}}{\alpha_{P(\alpha)}} \alpha_{P(z)} \quad(|z| \leq 1)$.

First, let us consider the $\alpha$-dec pgf's. The factor $P_{\alpha}$ in (3.1.2) is uniquel.y determined by $P$, as in a neighbourhood of zero we have

$$
\text { (3.1.5) } \quad P_{\alpha}(z)=P(\alpha) P(z) / P(\alpha z)
$$

For a general (not necessarily $\alpha-$ dec) pgf $P$ with $P(0)>0$, the function in the right-hand side of (3.1.5) is always defined in a neighbourhood of zero, and throughout sections 1 and 2 we shall denote this function by $P_{Q}$. Applying the mapping $\mathrm{P} \rightarrow \mathrm{P}_{\alpha}$ to $\mathrm{P}_{\beta}$, we see that, with an obvious notation,

$$
(3.1 .6) \quad\left(\mathrm{P}_{\beta}\right)_{\alpha}=\left(\mathrm{P}_{\alpha}\right)_{\beta} \quad(0<\alpha<1,0<\beta<1) .
$$

In view of lemma 1.3 .4 we have the following criterion for a pgf to be $\alpha$ dec.

LEMMA 3.1.3. If $0<\alpha<1$ and if $\mathrm{P}$ is a pgf with $\mathrm{P}(0)>0$, then $\mathrm{P}$ is $\alpha$-dec iff $P_{\alpha}$ is abs mon.

Looking for a general representation of $\alpha$-dec pgf's, we solve (3.1.2) for $P$ and we see that $P$ has the form of an infinite product. We now introduce the following notation: if $P$ is a pgf with $P(0)>0$, then 
(3.1.7) $\prod_{\alpha} P(z):=\prod_{k=0}^{\infty} P\left(\alpha^{k} z\right) / P\left(\alpha^{k}\right) \quad(0<\alpha<1)$.

It is not difficult to prove that this infinite product is absolutely and uniformly convergent in $|z| \leq 1$, and that $\pi_{\alpha} P$ is again a pgf, with

(3.1.8) $\quad \Pi_{\beta}\left(\Pi_{\alpha} P\right)=\Pi_{\alpha}\left(\Pi_{\beta} P\right) \quad(0<\alpha<1 ; 0<\beta<1)$.

Furthermore it is seen that $\pi_{\alpha}{ }{ }$, defined similarly as $(3.1 .7)$, is absolute$I_{y}$ and uniformly convergent in some neighbourhood of zero, with

(3.1.9) $\quad \pi_{\alpha} P_{\beta}=\left(\pi_{\alpha} P\right)_{\beta}$ and $\pi_{\alpha} P_{\alpha}=P$.

Now the following representation lemma for $\alpha$-dec pgf's is easily verified.

LEMMA 3.1.4. If $0<\alpha<1$ and if $P$ is a pgf with $P(0)>0$, then $P$ is a $\alpha$-dec iff $P$ has the form $P=\pi$, , where $Q$ is a $p g f$. The representation is unique: $Q=P_{\alpha}$

Turning to the $\alpha$-fact case, we give the following characterization.

LEMMA 3.1.5. Let $0<\alpha<1$ and let $P$ be a pgf with $P(0)>0$. Then $P$ is $\alpha-$ fact iff there exists a pgf 2 with $Q(0)>0$ such that

$(3.1 .10) \quad P_{\alpha}=Q_{\alpha}^{2}$ or, equivalently, iff $\pi{ }^{2^{2}}$ is $\alpha$-dec; in this case the factor $\alpha^{\alpha}$ of $P$ is gi-
ven by

(3.1.11) $\quad \alpha_{P(z)}=\pi \alpha^{2^{2} \alpha}(z)=\prod_{k=0}^{\infty} \frac{P\left(\alpha^{2 k} z\right) / P\left(\alpha^{2 k}\right)}{P\left(\alpha^{2 k+1} z\right) / P\left(\alpha^{2 k+1}\right)}$.

PROOF. Let $P$ be $\alpha$-fact. Then, iterating $(3.1 .4$ ) once, we get

$$
\alpha_{\mathrm{P}(z)}=\mathrm{P}(\mathrm{z}){ }^{\alpha} \mathrm{P}(\alpha) /{ }^{\alpha} \mathrm{P}(\alpha z)=\alpha_{\mathrm{P}\left(\alpha^{2} \mathrm{z}\right) \mathrm{P}(\mathrm{z}) / \mathrm{P}(\alpha z)}
$$

From this and the fact that $P(\alpha)={ }^{\alpha} \mathrm{P}\left(\alpha^{2}\right)$ (take $z=\alpha$ in $(3.1 .4)$ ), we obtain (3.1.10) with $Q={ }^{\alpha}$. By (3.1.9) it now follows that

$$
\text { (II }{ }_{\alpha} 2_{\alpha}^{P}=\pi \alpha_{\alpha} 2_{\alpha}^{P}=\pi 2_{\alpha}^{2}{ }^{2}=Q \text {. }
$$

and hence by lenma $3.1 .3 \pi \alpha^{2}{ }^{P}$ is $\alpha$-dec. 
Conversely, let $\pi_{\alpha} 2^{P}$ be $\alpha-$ dec. Then $Q:=\pi_{\alpha^{2}}{ }^{P} \alpha$ is a pgf, for which

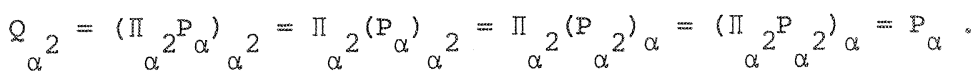

As $P(\alpha)=Q\left(\alpha^{2}\right)$, it follows that for all $\mathrm{n} \in \mathbb{N}$

$$
\begin{aligned}
P(z) & =\left\{Q(z) / Q\left(\alpha^{2} z\right)\right\} P(\alpha z)=P\left(\alpha^{n} z\right) \prod_{k=0}^{n-1} Q\left(\alpha^{k} z\right) / Q\left(\alpha^{k+2} z\right)= \\
& =P\left(\alpha^{n} z\right) \frac{Q(z) Q(\alpha z)}{Q\left(\alpha^{n} z\right) Q\left(\alpha^{n+1} z\right)} .
\end{aligned}
$$

Letting $n \rightarrow \infty$, we get

$$
P(z)=P(0) Q(z) Q(\alpha z) / Q(0)^{2}
$$

from which it is seen that $P(0) / Q(0)^{2}=Q(\alpha)$. It follows that $P$ is $\alpha$-fact with factor $\alpha_{\mathrm{P}}=\mathrm{Q}$.

Thus we have shown that the factor ${ }^{\alpha} \mathrm{p}$ of an $\alpha$-fact pgf $\mathrm{p}$ is uniquely determined by $P$. Furthermore it follows that the class of $\alpha$-dec pgf's is a proper subset of the class of a-fact pgf's.

LEMMA 3.1.6. If a pgf $P$ with $P(0)>0$ is $\alpha$-dec, then $P$ is $\alpha$-fact. In fact, $P$ is $\alpha$-dec iff $P$ is $\alpha$-fact with $\alpha^{2}$-dec factor ${ }^{\alpha} p$.

PROOF. Let $P$ be $\alpha$-dec. Then $P_{\alpha}$, and hence $\pi \alpha^{2}{ }^{P}$ r is a pgf, so that $\pi 2^{P}$ is $\alpha$-dec. By lemma 3.1 .5 it now follows that $P$ is $\alpha$-fact and its factor $\alpha_{\mathrm{P}}=\pi \alpha^{2}{ }^{\mathrm{P}} \alpha$ is $\alpha^{2}$-dec because of $(3.1 .12)$.

Conversely, if $P$ is $\alpha$-fact with $\alpha^{2}$-dec factor $\alpha_{P}$, then by $(3.1 .10)$ $P_{\alpha}=\left({ }^{\alpha} P\right)_{\alpha}{ }^{2}$ is a pgf, and hence $P$ is $\alpha$-dec.

From lemma 3.1 .4 and (3.1.4) it is evident that the classes of $\alpha$-dec and a-fact pgf"s are too large to have much structure. Therefore we want to re-strict them, and a first way to do so is by imposing conditions on the factors $P_{\alpha}$ and $\alpha_{P}$, respectively.

The most obvious condition is the inf div of $\mathrm{p}_{\alpha}$ and $\alpha_{\mathrm{p}}$. It turns out, however, that for all $\alpha \in(0,1)$ we then get the same class, namely $C_{1}$, both in the $\alpha$-dec and $\alpha$-fact case. 
THEOREM 3.1.7. Let $\mathrm{P}$ be a pgf with $\mathrm{P}(0)>0$. Then

(i) $P \in C_{1}$ iff for some, and then for all, $\alpha \in(0,1)$ is $\alpha$-dec with factor $P_{\alpha} \in C_{1} ;$

(ii) $\mathrm{P} \in \mathrm{C}_{1}$ iff for some, and then for all, $\alpha \in(0,1) \mathrm{P}$ is $\alpha$-fact with fac$\operatorname{tor} \alpha_{P}^{\alpha} \in C_{1}$.

PROOF. First, let $\mathrm{p} \in \mathrm{C}_{1}$. In lemma 2.3 .6 it is shown that then for all $\alpha \in(0,1) \mathrm{P}_{\alpha}$ is an inf div pgf. Hence for all $\alpha \in(0,1) \mathrm{P}$ is $\alpha$-dec with fac$\operatorname{tor} \mathrm{P}_{\alpha} \in \mathrm{C}_{1}$. Now, by lemma 3.1 .6 it follows that $\mathrm{P}$ is also $\alpha-$ fact for all $\alpha \in(0,1)$, while in view of $(3.1 .11)$ the factor $\alpha_{p}$ is the limit of a sequence of inf div pge's, so by the closure theorem $\alpha_{p} \in C_{1}$.

Next, let $P$ be $\alpha$-dec with factor $P_{\alpha} \in C_{1}$, for a fixed $\alpha \in(0,1)$. Then applying the closure theorem once more, from lemma 3.1 .4 we see that $P \in C_{1}$. Finally, if $P$ is $\alpha$-fact with factor $\alpha_{P} \in C_{1}$ for a fixed $\alpha \in(0,1)$, then the inf div of $\mathrm{p}$ immediately follows from $(3.1 .4)$.

Next, let us consider the cases that $\mathrm{P}_{\alpha}$ and $\alpha_{\mathrm{P}}$ are compound geometric. In view of $(3.1 .1)$ we have

(3.1.13) $\mathrm{P} \in \mathrm{C}_{\alpha} \Leftrightarrow \mathrm{P}$ a-dec with $\mathrm{P}_{\alpha} \in \mathrm{C}_{0}$

Using this, one easily verifies that the classes $C_{\alpha}$ can also be obtained as follows:

(3.1.14) $P \in C_{\alpha} \Leftrightarrow P$ a-fact with ${ }^{\alpha} P \in C_{\alpha}^{2}$.

Now, as for $0<\alpha<1 \quad C_{0}$ is a proper subset of $C_{\alpha}$ (cf. section 2.4), from (3.1.14) it follows that the question that led us to consider the $\alpha$-fact

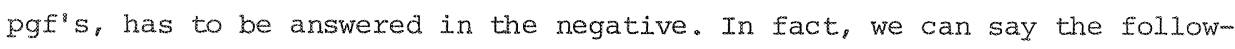
ing about the set of $\alpha$-fact pgf's with compound geometric factor ${ }^{\alpha}$.

LEMMA 3.1.8. If $0<\alpha<1$, then $C_{0} \varsubsetneqq\left\{\alpha\right.$-fact $\left.P \mid \alpha_{P} \in C_{0}\right\} \varsubsetneqq C_{\alpha}$. PROOF. In view of $(3.1 .14)$ we only need to prove the first part of the lemma. So let $\mathrm{P} \in \mathrm{C}_{\mathrm{O}}$. Then by theorem 3.1 .7 (ii) $\mathrm{P}$ is a-fact, and, as from corollary 2.4 .7 we see that

$$
{ }_{\alpha^{2}}{ }^{P} \in C_{\alpha}{ }^{2} \subset C_{\alpha}
$$

it follows from (3.1.13) that the factor $\left.\alpha_{p}=\pi_{\alpha}{ }_{2}{ }^{P}={ }_{\alpha}{ }^{P}\right)_{\alpha}$ is in $C_{0}$. 
Now, take $Q(z):=(1-p) /(1-p z)$, and define the pgf $P$ by

$$
P(z):=Q(z) Q(\alpha z) / Q(\alpha)
$$

then $P$ is $\alpha$-fact with factor $\alpha_{P}=Q \in C_{0}$. In view of (2.4.27), however, we see that $P \in C_{\alpha} \backslash \underset{\beta<\alpha}{U} C_{\beta}$, and hence $P \& C_{0}$.

of course, many other conditions might be imposed on $\mathrm{P}_{\alpha}$ or ${ }^{\alpha} \mathrm{p}$ to obtain subclasses of the classes of $\alpha-d e c$ and $\alpha$-fact pgf's. We mention one result: the classes $A_{\alpha, \beta}$ say, of $\alpha$-dec pgf's with factor $P_{\alpha} \in C_{\beta}$ define a classification of $C_{1}: A_{\alpha, \beta}$ is nondecreasing in both $\alpha$ and $\beta$, with

$$
A_{\alpha, \beta}=A_{\beta, \alpha} \supset C_{\alpha} \cup C_{\beta}
$$

as can be seen from the following charactexization:

$$
\mathbb{P} \in A_{\alpha, \beta} \Leftrightarrow 1-\frac{P(\alpha z) P(\beta z)}{P(z) P(\alpha \beta z)} \text { abs mon. }
$$

A second way to restrict the classes of $\alpha-d e c$ and $\alpha$-fact pgf's is to require $\alpha$-dec and $\alpha$-fact for $\alpha Z z \alpha \in(0,1)$. This is suggested by a notational analogy to the so-called self-decomposable chf's: a chf $\tilde{F}$ is said to be self-decomposable (self-dec) if for every $\alpha \in(0,1)$ there exists a chf $\widetilde{F}_{\alpha}$ such that $(3.1 .15) \quad \tilde{F}(t)=\widetilde{F}(\alpha t) \tilde{F}_{\alpha}(t) \quad(t \in \mathbb{I R})$

These chf's were introduced by Lêvy and Khintchine (cf. Lévy (1937)). For a survey of their main properties we refer to Lukacs (1970), ch. 5. Hexe we only mention that the class of self-dec distributions contains the wellknown stable distributions and is itself a proper subclass of the class of all inf div distributions.

\subsection{Totally decomposable and totally factorizable lattice distributions}

DEFINITION 3.2.1. Let $P$ be a pgf with $P(0)>0$. Then $P$ is said to be totalZy decomposable (tot dec) if $\mathrm{P}$ is $\alpha$-dec for all $\alpha \in(0,1)$. Similarly, $P$ is callea totally factorizable (tot fact) if $P$ is $\alpha$-fact for all $\alpha \in(0,1)$.

We start with studying the tot dec pgf"s. From theorem 3.1.7(i) we know that every inf div pgf is tot dec. It will turn out that the converse is also true. Though simpler proofs exist (to be given later), we shall. show this in a way, very similar to the proof of the inf div of a self-dec chf (cf. 
Lukacs (1970), ch. 5), thus showing that the analogy, noted at the end of section 1, is not merely notational. Proceeding in this way, we first prove the following lemma.

LEMMA 3.2.2. A tot dec pgf has no zeros in the closed unit disk $|z| \leq 1$.

PROOF. We need the following inequality:

(3.2.1) $|Q(\alpha z)| \leq|Q(z)|+1-Q(\alpha) \quad$ (Q is pgf; $0<\alpha<1 ;|z| \leq 1)$,

which can be proved as follows:

$$
\begin{aligned}
|Q(\alpha z)| & \leq|Q(z)|+|Q(z)-Q(\alpha z)| \leq|Q(z)|+\sum_{n=0}^{\infty} q_{n}\left(1-\alpha^{n}\right)|z|^{n} \leq \\
& \leq|Q(z)|+1-Q(\alpha) .
\end{aligned}
$$

Suppose the assertion of the lemma not to be true. Then we can find $\rho \in(0,1]$ and $z_{0} \in \notin$ with $\left|z_{0}\right|=\rho$ such that

$$
\mathrm{P}\left(z_{0}\right)=0 \text { and } \mathrm{P}(z) \neq 0 \quad(|z|<\rho) .
$$

From (3.1.2) it now follows that for all $\alpha \in(0,1)$ the factor $P_{\alpha}$ of $P$ satisfies

$$
\mathrm{P}_{\alpha}\left(\mathrm{z}_{0}\right)=0 \text { and } \mathrm{P}_{\alpha}(z) \neq 0 \quad(|z|<\rho) .
$$

As $\left|s_{z z}\right|<\rho$, we have on the one hand

$$
\lim _{\alpha \uparrow 1} P_{\alpha}\left(3_{\Sigma} z_{0}\right)=\lim _{\alpha \uparrow 1} P(\alpha) P\left(3_{\Sigma} z_{0}\right) / P\left(s_{\Sigma} \alpha z_{0}\right)=1
$$

whereas on the other hand from the inequality (3.2.1) it follows that

$$
\left|P_{\alpha}\left(\frac{1}{2} z_{0}\right)\right| \leq\left|P_{\alpha}\left(z_{0}\right)\right|+1-P_{\alpha}\left(\frac{1}{2}\right) \leq 1-P_{\alpha}(0)=1-P(\alpha) .
$$

which tends to zero as $\alpha \uparrow 1$. Thus we have obtained a contradiction, and the lemma is proved.

In the self-decomposable case one makes use of the theorem (see e.g. Gnedenko \& Kolmogorov (1949)) that, if a chf $\varphi$ can be written as

$$
\varphi(t)=\lim _{n \rightarrow \infty} \prod_{k=1}^{n} \varphi_{n, k}(t) \quad(t \in \mathbb{R}),
$$

where the $\varphi_{n, k}$ 's form an infinitesimal system of chf's, then $\varphi$ is inf div. 
Here a system $\left(\varphi_{n, k} \mid n \in \mathbb{N} ; k \in\{1, \ldots, n\}\right)$ of chf's is called infinitesimal if

$$
\lim _{n \rightarrow \infty} \sup _{1 \leq k \leq n}\left|\varphi_{n, k}(t)-1\right|=0 \quad(t \in \mathbb{R}) .
$$

Translating this to pgf"s, we obtain the following result.

THEOREM 3.2.3. A pgf $P$, with $P(0)>0$, is inf div iff there exist pgf's $P_{n, k}(n \in \mathbb{N} ; k \in\{1, \ldots, n\})$ satisfying

$$
\text { (3.2.2) } \lim _{n \rightarrow \infty} \inf _{1 \leq k \leq n} P_{n, k}(0)=1 \text {, }
$$

such that

$$
\text { (3.2.3) } P(z)=\lim _{n \rightarrow \infty} \prod_{k=1}^{n} P_{n, k}(z) \quad(|z| \leq 1) .
$$

We are now ready to prove the announced result.

THEOREM 3.2.4. A pgf $P$, with $P(0)>0$, is inf div iff $P$ is tot dec.

PROOF. As already noted, in view of theorem 3.1.7(i) we only need to show that a tot dec pgf is inf div. So let $\mathrm{P}$ be tot dec. Then by lemma 3.2 .2 it is seen that for the factors $\mathbb{P}_{\alpha}(0<\alpha<1)$ relation (3.1.5) holds for all $|z| \leq 1$. If we define the pgf"s $\mathrm{P}_{\mathrm{n}, \mathrm{k}}$ for $\mathrm{n} \in \mathbb{N}$ and $\mathrm{k} \in\{1, \ldots, \mathrm{n}\}$ by

$$
P_{n, k}(z):=P_{(k-1) / k}\left(\frac{k}{n} z\right) / P_{(k-1) / k}\left(\frac{k}{n}\right) \quad(|z| \leq 1),
$$

then it follows that

$$
P_{n, k}(z)=\frac{P\left(\frac{k}{n} z\right) / P\left(\frac{k}{n}\right)}{P\left(\frac{k-1}{n} z\right) / P\left(\frac{k-1}{n}\right)} \quad(|z| \leq 1) .
$$

and hence

$$
P(z)=\prod_{k=1}^{n} p_{n, k}(z) \quad(n \in \mathbb{N} ;|z| \leq 1) .
$$

Now from theorem 3.2 .3 we obtain the inf div of $P$, as soon as we have proved (3.2.2), which in our case is equivalent to

(3.2.4) $\lim _{n \rightarrow \infty} \inf _{1 \leq k \leq n} P\left(\frac{k-1}{n}\right) / P\left(\frac{k}{n}\right)=1$. 
Because of the uniform continuity of $\mathrm{p}$ on $[0,1]$, for all $\varepsilon>0$ one can find $\mathbb{N} \in \mathbb{N}$ such that

$$
\forall_{\mathrm{n} \geq \mathbb{N}} \forall_{\mathrm{k} \in\{1, \ldots, \mathrm{n}\}}\left|\mathrm{P}\left(\frac{\mathrm{k}-1}{\mathrm{n}}\right)-\mathrm{P}\left(\frac{\mathrm{k}}{\mathrm{n}}\right)\right|<\varepsilon,
$$

or

$$
\forall_{\mathrm{n} \geq \mathbb{N}} \forall_{\mathrm{k} \in\{1, \ldots, \mathrm{n}\}}\left|\mathrm{P}\left(\frac{\mathrm{k}-1}{\mathrm{n}}\right) / \mathrm{P}\left(\frac{\mathrm{k}}{\mathrm{n}}\right)-1\right|<\varepsilon / \mathrm{P}(0)
$$

From this (3.2.4), and hence the theorem, immediately follows.

The following two corollaries are obtained by using theorem 3.1 .7 (i) (or lemma 2.3 .6 ) and lemma 3.1 .3 , respectively.

COROLLARY 3.2.5. The factors $\mathrm{p}_{\alpha}(0<\alpha<1)$ of a tot dec pgf $\mathrm{p}$ are all inf div.

COROLLARY 3.2.6. Let $P$ be a pgf with $P(0)>0$. Then $P \in C_{1}$ iff for all $\alpha \in(0,1)$, or, equivalently, for all $\alpha \in(1-\varepsilon, 1)$ (some $\varepsilon>0$ ), the function $p(z) / p(\alpha z)$ is abs mon.

The characterization of $C_{1}$, just given, can also be proved as follows. The necessity of the condition has already been shown in lemma 2.3.6. Suppose therefore that $\varepsilon>0$ and that $P(z) / P(\alpha z)$ is abs mon for all $\alpha \in(1-\varepsilon, 1)$. As $P(z) / P(\alpha z)$ is equal to 1 for $z=0$, for all $\alpha \in(1-\varepsilon, 1)$ the function

$$
(3.2 .5) \quad \mathbb{T}_{\alpha}(z):=\frac{1}{1-\alpha} \frac{1}{z}\{P(z) / P(\alpha z)-1\}
$$

is abs mon. But then so is the following function:

$$
\lim _{\alpha \uparrow 1} T_{\alpha}(z)=\lim _{\alpha \uparrow 1} \frac{1}{P(\alpha z)} \frac{P(z)-P(\alpha z)}{z-\alpha z}=P^{\prime}(z) / P(z)
$$

from which by theorem 1.5 .3 it follows that $\mathrm{P} \in \mathrm{C}_{1}$.

In view of lemma 3.1 .3 this alternative proof now yields a simplex proof of theorem 3.2.4. Furthermore we note that another proof of this theorem can be given along the lines of the proofs of theorems 3.3 .3 and 3.4 .5 .

The $R_{\alpha}-$ function of a pgf $P$ can be expressed in the function $T_{\alpha}$ from $(3.2 .5)$ as follows:

(3.2.6) $\quad R_{\alpha}(z)=T_{\alpha}(z) P(\alpha z) / P(z)$.

Now, apart from (3.1.13), from this relation it can be clearly seen that $C_{\alpha}$ is a subclass of the class of $\alpha$-dec pgf"s, the latter being characterized by the 
abs mon of $\mathrm{T}_{\alpha}$.

Finally, denoting the coefficients in the power-series expansion of $T_{\alpha}$ by $t_{n}(\alpha)\left(n \in \mathbb{N}_{0}\right)$, from corollary 3.2 .6 we get the following characterization of $C_{1}$ by means of recurrence relations (cf. those defining the classes $C_{\alpha}$ ).

LEMMA 3.2.7. A lattice distribution $\left\{\mathrm{p}_{\mathrm{n}}\right\}_{0}^{\infty}$, with $\mathrm{p}_{0}>0$, is in $\mathrm{C}_{1}$ iff for all $\alpha \in(0,1)$ (or $\alpha \in(1-\varepsilon, 1)$, some $\varepsilon>0$ ) there exist nonnegative quantities $t_{n}(\alpha) \quad\left(n \in \mathbb{N}_{0}\right)$ such that

(3.2.7) $\frac{1-\alpha^{n+1}}{1-\alpha} p_{n+1}=\sum_{k=0}^{n} \alpha^{k} p_{k} t_{n-k}(\alpha) \quad\left(n \in \mathbb{N}_{0}\right)$.

We now turn to the tot fact pgf's. By theorem 3.1.7(ii) we know that all inf div pgf's are tot fact. Although an $\alpha$-fact pgf is more general than an $\alpha$-dec pgf (cf. lemma 3.1.6), and the tot dec pgf's coincide with the inf divpgf's, all tot fact pgf's might be inf div. In order to decide this, we study the tot fact pgf's in more detail, and start with the behaviour of the factor $\alpha_{\mathrm{P}}$ for $\alpha \uparrow 1$ and $\alpha \neq 0$.

THEOREM 3.2.8. A tot fact pgf $\mathrm{P}$ is 2-div; in fact, the factor ${ }^{\alpha} \mathrm{P}$ of $\mathrm{P}$ satisfies

(3.2.8) $\lim _{\alpha \uparrow 1} \alpha p\left(\alpha^{\mathrm{n}} z\right)=\mathrm{P}(z)^{\frac{3}{2}} \quad\left(|z| \leq 1 ; \mathrm{n} \in \mathbb{N}_{0}\right)$,

and furthermore

(3.2.9) $\lim _{\alpha \downarrow 0} \alpha_{P(z)}=P(z)$ and $\lim _{\alpha \downarrow 0} \alpha_{P\left(\alpha^{n} z\right)=P(0)}(|z| \leq 1 ; n \in \mathbb{N})$.

PROOF. Let $\mathrm{P}$ be tot fact. Taking $\mathrm{z}=\alpha$ in (3.1.4), we get ${ }^{\alpha} \mathrm{P}\left(\alpha^{2}\right)=\mathrm{P}(\alpha)$,

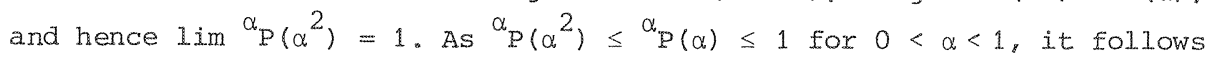
that $\lim \alpha_{\mathrm{P}(\alpha)}^{\alpha \uparrow 1}=1$, and hence

$\alpha \uparrow 1$

$$
\lim _{\alpha \uparrow 1} \alpha_{P(z)}{ }^{\alpha} \mathrm{P}(\alpha z)=\mathrm{P}(z) \quad(|z| \leq 1) .
$$

Now, let $\left\{p_{k}\right\}_{0}^{\infty}$ and $\left\{p_{k}(\alpha)\right\}_{0}^{\infty}$ be the lattice distributions with pgf $P$ and ${ }^{\alpha} p_{\text {, }}$ respectively. Then by the continuity theorem for pgf's (theorem 1.3.5) we have 
(3.2.10) $\lim _{\alpha \uparrow 1} \sum_{j=0}^{k} \alpha^{j} p_{j}(\alpha) p_{k-j}(\alpha)=p_{k} \quad\left(k \in \mathbb{N}_{0}\right)$.

Taking $k=0$ in this relation, we see that $\lim _{\alpha \uparrow 1} p_{0}(\alpha)=p_{0}^{1 / 2}$. Suppose that $p_{n}(1):=\lim _{\alpha \uparrow 1} p_{n}(\alpha)$ exists for $n=1, \ldots, k-1$; then from $(3.2 .10)$ it follows that also

$$
\lim _{\alpha \uparrow 1} p_{k}(\alpha)=\frac{1}{2} p_{0}^{-\frac{1}{2}}\left\{p_{k}-\sum_{j=1}^{k-1} p_{j}(1) p_{k-j}(1)\right\}
$$

exists, and hence (mathematical induction): $p_{k}(1)=\lim p_{k}(\alpha)$ exists for all $k \in \mathbb{N}_{0}$, while $\left\{\mathrm{p}_{\mathrm{k}}(1)\right\}_{0}^{\infty}$ satisfies

$$
\sum_{j=0}^{k} p_{j}(1) p_{k-j}(1)=p_{k} \quad\left(k \in \mathbb{N}_{o}\right)
$$

Applying the continuity theorem in the other direction, we conclude that

$$
\lim _{\alpha \uparrow 1} \alpha_{P(z)}=Q(z) \quad(|z| \leq 1),
$$

where $Q(z):=\sum p_{n}(1) z^{n}$ is a pgf satisfying $Q(z)^{2}=P(z)$. Hence $P$ is 2 -div, and $(3.2 .8)$ is proved for $n=0$. Replacing $z$ by $\alpha^{n} z$ in $(3.1 .4)$, we get

(3.2.11) $\quad \alpha_{P}\left(\alpha^{n+1} z\right)=\alpha_{P}(\alpha) P\left(\alpha^{n} z\right) / \alpha_{P}\left(\alpha^{n} z\right)$

from which by use of mathematical induction (3.2.8) follows for all $\mathrm{n} \in \mathbb{N}$. Finally, let $\alpha+0$. Taking $z=\alpha$ and $z=\alpha^{2}$ in (3.1.4) successively, one sees that

$$
\lim _{\alpha \downarrow 0} \alpha_{P}\left(\alpha^{2}\right)=\mathrm{P}(0) \text { and } \lim _{\alpha \downarrow 0} \alpha_{\mathrm{P}\left(\alpha^{3}\right) /{ }^{\alpha} \mathrm{P}(\alpha)=1 .}
$$

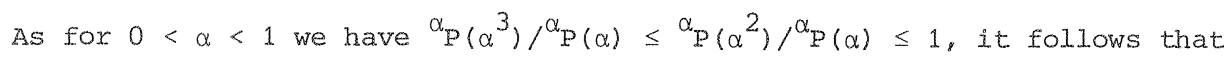

$$
\lim _{\alpha \downarrow 0} \alpha_{P}\left(\alpha^{2}\right) / \alpha_{P}(\alpha)=1
$$

and hence

$$
\lim _{\alpha \downarrow 0} \alpha_{P(\alpha)}=P(0)
$$

Observing that for $0<z \leq 1$ and $0<\alpha \leq z$ we have ${ }^{\alpha} P\left(\alpha^{2}\right) \leq{ }^{\alpha} P(\alpha z) \leq{ }_{P}(\alpha)$, we conclude that for all $z \in(0,1]$, and hence by the continuity theorem for all $|z| \leq 1$ 


$$
\lim _{\alpha \downarrow 0} \alpha_{p}(\alpha z)=p(0)
$$

The second part of (3.2.9) is now easily obtained from (3.2.11) by mathematical induction, while the first part follows from $(3.1 .4)$.

In view of the preceding theorem one might conjecture that the tot fact pgf's coincide with the 2-div ones. Consider, however, the pgf $P(z)=$ $=(1+z)^{2} / 4$; obviously, $P$ is 2 -div, but $P$ is not $\alpha$-fact for any $\alpha \in(0,1)$. In fact, if $P$ would be $\alpha$-fact, then $\alpha_{P}$ is necessarily of the form $\alpha_{P}(z)=$ $=c_{\alpha}\left(1+\gamma_{\alpha} z\right)$ with $c_{\alpha}>0, \gamma_{\alpha}>0$. But if we look at the zeros of $P$, then we see that $\gamma_{\alpha}$ has to satisfy: $\gamma_{\alpha}=\alpha \gamma_{\alpha}=1$, which is impossible for $\alpha \in(0,1)$. A similar observation with respect to the zeros of $\mathrm{P}$ yields a property that the tot fact pgf's share with the inf div ones (cf. theorem 1.4.6).

THEOREM 3.2.9. If $\mathrm{P}$ is a tot fact pgf, then the corresponding lattice distribution $\left\{p_{n}\right\}$ has an infinite support.

PROOF. Let $P$ be tot fact and suppose that the support of $\left\{p_{n}\right\}_{0}^{\infty}$ is finite. Then $P$, and its factors $\alpha_{\mathrm{p}}(0<\alpha<1)$, are polynomials. Let $z_{0}$ be a zero of the factor ${ }^{\frac{1}{2}} \mathrm{p}$, then, as $\mathrm{p}_{0}>0, \mathrm{z}_{0} \neq 0$, and from (3.1.4), which now holds for all $z \in \mathbb{Q}$, it follows that $P\left(z_{0}\right)=P\left(2 z_{0}\right)=0$. Taking $a=1 / k$ $(k=2,3 \ldots)$ in $(3.1 .4)$, we then see that $1 / k_{P}\left(z_{0}\right)=0$ or $1 / k_{P}\left(z_{0} / k\right)=0$, and hence

$$
\mathrm{P}\left(\mathrm{k} \mathrm{z}_{\mathrm{O}}\right)=0 \text { or } \mathrm{P}\left(\mathrm{z}_{\mathrm{O}} / \mathrm{k}\right)=0 \quad(\mathrm{k} \in \mathbb{N}) .
$$

As $z_{0} \neq 0$, we have thus obtained an infinite sequence of different zeros of $P$, which contradicts the fact that $P$ is a polynomial. It follows that the support of $\left\{\mathrm{p}_{\mathrm{n}}\right\}$ has to be infinite.

REMARK 3.2.10. If $P$ is $\alpha$-fact for only a finite number of $\alpha$ 's, then the support of $\left\{p_{n}\right\}$ may be finite. To show this, let $\alpha_{1}, \ldots, \alpha_{n} \in(0,1)$, and let $p_{n}$ be the pgf that is equal to a polynomial with $A_{n}$ as its set of zeros, where $A_{n}$ is recursively defined by

$$
A_{k}:=A_{k-1} \cup\left\{\alpha_{k}^{-1} x \mid x \in A_{k-1}\right\} \quad(k \in\{1, \ldots, n\}), A_{0}:=\{-1\} .
$$

Then $P_{n}$ is of degree $2^{n}$, and it is easily seen that $P_{n}$ is $\alpha_{k}$-fact for all $k \in\{1, \ldots, n\}$. 
Apart from being $2-\mathrm{div}$ and having infinite support, the tot fact pgf's share a third property with the inf div pgf's, viz. having no zeros in the open unit disk $|z|<1$ (cf. corollary 1.5.2). It is not clear if a tot fact pgi may have zeros for $|z|=1$.

THEOREM 3.2.11. If $P$ is a tot fact pgf, then $P(z) \neq 0$ for all $|z|<1$.

PROOF. Let $P$ be tot fact, and suppose the assertion not to be true. Then we can find $\rho \in(0,1)$ and $z_{0} \in \phi$ with $\left|z_{0}\right|=\rho$, such that

$$
P\left(z_{0}\right)=0 \text { and } P(z) \neq 0 \quad(|z|<\rho)
$$

From (3.1.4) it follows that for all $\alpha \in(0,1)$ the factor $\alpha_{p}$ of $p$ satisfies

$$
{ }^{\alpha} \mathrm{p}\left(z_{0}\right)=0 \text { and }{ }^{\alpha} \mathrm{p}(z) \neq 0 \quad(|z|<\rho) \text {. }
$$

Now, take $\alpha \in(\rho, 1)$, then $P\left(z_{0} / \alpha\right)$ is well defined, and according to (3.1.4)

$$
\mathrm{P}\left(z_{0} / \alpha\right)={ }^{\alpha} \mathrm{P}\left(z_{0} / \alpha\right)^{\alpha} \mathrm{P}\left(z_{0}\right) /{ }^{\alpha} \mathrm{P}(\alpha)=0
$$

hence

$$
P\left(\gamma z_{0}\right)=0 \quad(1 \leq \gamma \leq 1 / \rho)
$$

However, as $P$ is analytic on $|z| \leq 1$, this would imply that $P \equiv 0$, so we have obtained a contradiction, and hence $P(z) \neq 0$ for all $|z|<1$.

If $P$ is tot fact, then the coefficients $p_{k}(\alpha)$, say, in the power-series expansion of the function $\alpha_{p}$, given by (3.1.11). have to be nonnegative for all $\alpha \in(0,1)$. Now, from $(3.1 .4)$ we easily see that

$(3.2 .12)\left[\forall{ }_{\alpha \in(0,1)} p_{2}(\alpha) \geq 0\right] \Leftrightarrow 4 p_{0} p_{2} \geq p_{1}^{2}$

We compare this condition with the condition $r_{1}(1) \geq 0$ (cf. corollary 1.5.5), necessary for $P$ to be inf div, for which we have

$$
r_{1}(1) \geq 0 \Leftrightarrow 2 p_{0} p_{2} \geq p_{1}^{2}
$$

As the latter condition is more restrictive and as the same phenomenon seems to occur at further coefficients, we are led to look for examples of tot fact pgf's that are not inf div.

To this end the following characterization of an $\alpha$-fact pgf (and hence of a tot fact pgf) is useful. 
LEMMA 3.2.12. Let $P$ be a pgf with $P(0)>0$, and define the sequence $\left\{a_{n}\right\}_{1}^{\infty}$ by

(3.2.13) $\log \{P(z) / P(0)\}=\sum_{n=1}^{\infty} a_{n} z^{n}$

Then for $0<\alpha<1 \mathrm{P}$ is $\alpha$-fact iff the function $Q^{(\alpha)}$, defined by

$(3.2 .14) \quad Q^{(\alpha)}(z):=\exp \left[\sum_{n=1}^{\infty} \frac{a_{n}}{1+\alpha^{n}} z^{n}\right]$,

is abs mon, in which case necessarily $Q^{(\alpha)}(z)=\alpha_{p}(z) / \alpha_{p}(0)$.

PROOF. Let $P$ be a pgf with $P(0)>0$, and let $0<\alpha<1$. Then, using (3.2.13), we can write

$$
P(z) / P(0)=\exp \left[\sum_{n=1}^{\infty} a_{n} z^{n}\right]=\exp \left[\sum_{n=1}^{\infty} \frac{a_{n}}{1+\alpha^{n}} z^{n}+\sum_{n=1}^{\infty} \frac{a_{n}}{1+\alpha^{n}}(\alpha z)^{n}\right] .
$$

from which it follows that $\mathrm{P}$ can be written as (cf. $(3.2 .14)$ )

$(3.2 .15) \quad P(z) / P(0)=Q^{(\alpha)}(z) Q^{(\alpha)}(\alpha z)$

Now, if $\mathrm{P}$ is $\alpha$-fact with factor $\alpha_{\mathrm{P}}$, then $\mathrm{P}(0)^{\alpha} \mathrm{P}(\alpha)=\left\{{ }^{\alpha} \mathrm{P}(0)\right\}^{2}$, which, together with (3.2.15), implies that $Q^{(\alpha)}(z)=\alpha_{P(z)} /^{\alpha} p(0)$; hence $Q^{(\alpha)}$ is abs mon.

Conversely, let $Q^{(\alpha)}$ be abs mon. Then similarly to the proof of lemma 1.3 .4 it follows from $(3.2 .15)$ that $Q^{(\alpha)}(z)$ is convergent for $|z| \leq 1$, with $Q^{(\alpha)}(1) Q^{(\alpha)}(\alpha)=1 / P(0)$. Now, defining $\alpha_{P(z)}:=Q^{(\alpha)}(z) / Q^{(\alpha)}(1)$, we see from (3.2.15) that $P$ is $\alpha$-fact with factor ${ }^{\alpha}$.

For a pgf $\mathrm{P}$ to be inf div it is necessary and sufficient that the quantities $a_{n}$, defined by (3.2.13), are all nonnegative (cf. theorem 1.5.3). Hence, in view of lemma 3.2 .12 , in ordex to obtain a tot fact pgf that is not inf div, we need an example of a power series $Q$ with at least one negative coefficient such that $\exp [Q(z)]$ is abs mon. The simplest example of this type is provided by a polynomial of degree 4 , as was shown by Lêvy (1937), ch. VII. We state his result as a lemma.

LERIMA 3.2.13. For given $\mathrm{a}>0, \mathrm{c}>0$ and $\mathrm{d}>0$ there exists a (unique) real number $m(a, c, d)>0$, such that $F(z):=\exp \left[a z-b z^{2}+c z^{3}+d z^{4}\right]$ i.s abs mon iff $b \leq m(a, c, d)$. Furthermore, in this case the pgf $P(z):=F(z) / F(1)$ is indecomposable iff $\mathrm{b}=\mathrm{m}(\mathrm{a}, \mathrm{c}, \mathrm{d})$. 
Now we are ready to prove the existence of tot fact pgf's that are not inf div.

THEOREM 3.2.14. The class $C_{1}$ is a proper subset of the class of tot fact pgf's.

PROOF. Choose arbitrary positive numbers $a_{1}, a_{3}$ and $a_{4}$, and define $m(\alpha)$ by

$$
m(\alpha):=m\left(a_{1} /(1+\alpha), a_{3} /\left(1+\alpha^{3}\right), a_{4} /\left(1+\alpha^{4}\right)\right) \quad(0 \leq \alpha \leq 1)
$$

(cf. the preceding lemma). It is easy to show that the function $m(a, c, d)$ is nondecreasing in $a, c$ and $d$, and hence $m(\alpha)$ is nonincreasing in $\alpha \in[0,1]$. Now, take $\varepsilon>0$ such that $\varepsilon \leq m(1)$, then $\varepsilon \leq m(0)$ and by lemma 3.2 .13 it follows that

$$
\text { (3.2.16) } P(z) / P(0)=\exp \left[a_{1} z-\varepsilon z^{2}+a_{3} z^{3}+a_{4} z^{4}\right]
$$

defines a pgf. As its $R_{1}$-function is not abs mon, we have (cf. theorem 1.5.3) $P \& C_{1}$. But by the monotonicity of $m(\alpha)$, we have

$$
\varepsilon /\left(1+\alpha^{2}\right)<\varepsilon \leq \mathrm{m}(1) \leq \mathrm{m}(\alpha) \quad(0<\alpha<1) .
$$

and hence, applying lemma 3.2 .13 once more, we see that

$$
\exp \left[\frac{a_{1}}{1+\alpha} z-\frac{\varepsilon}{1+\alpha^{2}} z^{2}+\frac{a_{3}}{1+\alpha^{3}} z^{3}+\frac{a_{4}}{1+\alpha^{4}} z^{4}\right]
$$

is an abs mon function for all $\alpha \in(0,1)$. From lemma 3.2.12 it now follows that $\mathrm{P}$ is tot fact, and thus the theorem is proved.

REMARK 3.2.15. In view of the preceding proof one can replace the condition $\varepsilon \leq \mathrm{m}(1)$ by the weaker condition

$$
\varepsilon \leq \inf _{0 \leq \alpha<1}\left(1+\alpha^{2}\right) m(\alpha) .
$$

It follows that if the function $m(\alpha)$ is such that

$$
2 m(1) \leq\left(1+\alpha^{2}\right) m(\alpha) \quad(0 \leq \alpha<1),
$$

then one could choose $\varepsilon=2 \mathrm{~m}(1)$, in which case the pgf $P$ from (3.2.16) is such that its factor $\mathrm{P}(z)^{\frac{1}{2}}$ is indecomposable (cf. the last part of lemma $3.2 .13)$. 
In order to construct real examples of tot fact pgf's that are not inf div, it is necessary to have an expression, or at least a positive lower bound, for the function $\mathrm{m}(\mathrm{a}, \mathrm{c}, \mathrm{d})$. Lévy does not give any information of this type, but Lukacs (1970), ch. 8 notes that if $Q(z):=1+a z-b z^{2}+c z^{3}+a z^{4}$ is such that $b \leq \frac{1}{2} a^{2}$ and $Q^{2}$ and $Q^{3}$ are abs mon, then exp[Q(z)] is abs mon. Elaborating this, one easily verifies the following lemma.

LEMMA 3.2.16. For given $\mathrm{a}>0, \mathrm{c}>0$ and $\mathrm{d}>0$ the function

$\exp \left[a z-b z^{2}+c z^{3}+d z^{4}\right]$ is abs mon, if $b \leq \min \left\{a^{2} / 3, c / a, a d /(2 c), c^{2} /(3 d)\right\}$.

It follows that, for instance, the function $P$ defined by

$$
P(z)=\exp \left[6 z-z^{2}+6 z^{3}+6 z^{4}-17\right]
$$

is a tot fact pgf that is not inf div.

To conclude this section, we return to chf's. Analogous to self-dec chf"s (cf. the end of section 1) we defined tot dec pgf's. Now, having introduced tot fact pgf's, we could reverse matters and consider totally factorizable chf's, i.e. chf's $\tilde{\mathrm{F}}$ that satisfy

$$
(3.2 .17) \quad \tilde{F}(t)=\tilde{F}_{\alpha}(\alpha t) \tilde{F}_{\alpha}(t) \quad(t \in \mathbb{R} ; 0<\alpha<1) \text {. }
$$

where $\tilde{F}_{\alpha}$ is a chf. or, in terms of $x v^{\prime} s: x$ is said to be tot fact if

$$
\text { (3.2.18) } \quad \mathrm{x} \stackrel{\mathrm{d}}{=} \mathrm{x}_{\alpha}+\alpha \mathrm{x}_{\alpha}^{8} \quad(0<\alpha<1)
$$

where $x_{\alpha}$ and $x_{\alpha}^{\prime}$ are independent $r v^{\prime} s$ with the same distribution. As an $\mathbb{N}_{0}^{-}$ valued $r v X \neq 0$ cannot satisfy $(3.2 .18)$, we see that an inf div chf is not necessarily tot fact (cf. theorem 3.1.7(ii)). Furthermore, proceeding in a way similar to lemmas 3.1 .5 and 3.1 .6 , we can show that the class of selfdec chf's is a proper subset of the class of tot fact chf" $s$, and the question arises whether the latter class is a subset of the class of inf div chf's, i.e. whether all tot fact chf's are inf div. We will not go further into this now. We only note that e.g. the rectangular distribution is $\alpha-$ fact in this sense for infinitely many $\alpha^{\prime}$; in fact, the chf of the rectangular distribution on $(-1,+1)$ can be written as (cf. Lukacs $(1970)$, ch. 6)

$$
\tilde{F}(t)=\frac{\sin t}{t}=\prod_{k=1}^{\infty} \cos \left(t / 2^{k}\right)
$$

from which it is seen that $\tilde{F}$ is $2^{-n}$-fact for all $n \in \mathbb{N}$. 


\subsection{Discrete self-decomposability and stability}

As noted at the end of section 1, a chf $\tilde{F}$ is said to be self-decomposable (self-dec) if $\widetilde{\mathrm{F}}$ satisfies

(3.3.1) $\tilde{\mathbb{F}}(t)=\tilde{F}(\alpha, t) \tilde{F}_{\alpha}(t) \quad(t \in \mathbb{R} ; 0<\alpha<1)$.

with $\widetilde{F}_{\alpha}$ a chf. For the corresponding $r v^{\prime} s$ this means that

(3.3.2) $\quad \mathrm{x} \stackrel{\mathrm{d}}{=} \alpha \mathrm{x}+\mathrm{x}_{\alpha} \quad(0<\alpha<1)$,

where $x$ and $x_{\alpha}$ are independent. Vervat (1978) considers equations of the form (3.3.2), where also the factor $\alpha$ is a $r v$. clearly, except $x \equiv 0$, no $\mathbb{N}_{O^{-}}$ valued $r v X$ can satisfy (3.3.2); in fact, all nondegenerate self-dec distributions are known to be absolutely continuous (cf. Fisz \& Varadarajan (1963)). Now in this section we propose analogues of the concepts of self-decomposability and stability (cf. (3.3.20)) for lattice distributions. A slightly condensed version of the results of the present section can be found in Steutel \& Van Harn (1978). It turns out that the discrete self-dec distributions and the discrete stable distributions share the basic properties with their continuous counterparts. The discrete self--dec distributions, for instance, are unimodal (cf. Wolfe (1971a) and Yamazato (1978)), and the dis-crete stable distributions are very similar to their continuous analogues on $(0, \infty)$.

Looking for analogues of $(3.3 .2)$ that operate within the set of $\mathbb{N}_{0}$-valued $r V^{\prime} \mathrm{s}$, we consider equations of the form

$$
\text { (3.3.3) } \quad \mathrm{x} \stackrel{\mathrm{d}}{=} \alpha \circ \mathrm{x}+\mathrm{x}_{\alpha} \quad(0<\alpha<1)
$$

where the operation 0 is such that $\alpha \circ x$ is an $\mathbb{N}$ o-valued $r v$. In terms of $\operatorname{pgf}^{8} \mathrm{~S}$ :

$$
\text { (3.3.4) } \mathrm{P}(\mathrm{z})=\left(\mathrm{T}_{\alpha} \mathrm{P}\right)(z) \mathrm{P}_{\alpha}(z) \quad(|z| \leq 1 ; 0<\alpha<1) .
$$

where the operator $\mathrm{T}_{\alpha}$ is such that $\mathrm{T}_{\alpha} \mathrm{P}$ is a pgf. Now we want to choose $\alpha \circ \mathrm{X}$ or $\mathrm{T}_{\alpha} \mathrm{p}$ in such a way that they have properties as in ordinary scalar multiplication. We mention three examples, which satisfy

$$
T_{0} P=1, T_{1} P=P, T_{\alpha}\left(T_{\beta} P\right)=T_{\alpha, \beta} P
$$


EXAMPLE 3.3 .1 .

(i)

$$
\begin{aligned}
& \left(T_{\alpha} P\right)(z)=P(\alpha z) / P(\alpha) \\
& \left(T_{\alpha} P\right)(z)=1-\alpha+\alpha P(z) \text {, or } \alpha \circ x=x_{1}+\ldots+x_{N} .
\end{aligned}
$$

where $\mathrm{P}(\mathrm{N}=1)=1-\mathrm{P}(\mathrm{N}=0)=\alpha, \mathrm{X}_{\mathrm{k}} \stackrel{\mathrm{d}}{=} \mathrm{X}(\mathrm{k} \in \mathbb{I N})$, all $r \mathrm{v}^{\prime} \mathrm{s}$ being independent.

$$
\left(T_{\alpha} P\right)(z)=P(1-\alpha+\alpha z) \text {, or } \alpha \circ X=N_{1}+\ldots+N_{X} \text {, }
$$

where $N_{k} \stackrel{d}{=} N(k \in \mathbb{N})$ with $N$ as in example (ii), all $r v^{8} s$ being independent.

It is easy to see that only examples (i) and (iii) satisfy

$$
T_{\alpha}(P Q)=\left(T_{\alpha} P\right)\left(T_{\alpha} Q\right)
$$

and that only examples (ii) and (iii) satisfy

and

$$
\mathrm{T}_{\alpha}(\gamma \mathrm{P}+(1-\gamma) Q)=\gamma \mathrm{T}_{\alpha} \mathrm{P}+(1-\gamma) \mathrm{T}_{\alpha} Q \quad(0 \leq \gamma \leq 1)
$$

$$
\left(T{ }_{\alpha} P\right)^{\prime}(1)=\alpha P^{*}(1)
$$

Thus, example (iii) seems to be most similar to ordinary scalar multiplication. Indeed, it will turn out that using this example in (3.3.4), we obtain a class of lattice distributions that can be considered as the lattice analogue of the class of self-dec distributions. When using example (i) in (3.3.4), we get the class $C_{1}$ (cf. theorem 3.2.4), and, as we shall see in the next section, the class of pgf's $P$ that satisfy (3.3.4) with $T_{\alpha}{ }^{P}$ given by example (ii), coincides with $C_{0}$.

DEFINITION 3.3.2. A pgf $P$, with $P(0)>0$, is said to be discrete self-decomposable if $\mathrm{P}$ satisfies

(3.3.5) $\quad \mathrm{P}(z)=\mathrm{P}(1-\alpha+\alpha z) \mathrm{P}_{\alpha}(z) \quad(|z| \leq 1 ; 0<\alpha<1)$, with $\mathrm{P}_{\alpha}$ a pgf.

REMARK 3.3.3. A relation that suggests the analogy of discrete self-dec pgf's to self-dec chf's in another respect, is the relation that $H(z):=$ $:=P(1-z)$ satisfies:

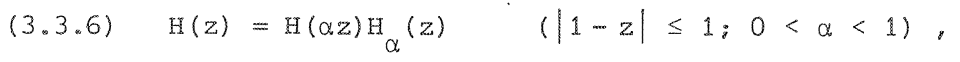

with $H_{\alpha}(z):=P_{\alpha}(1-z)$. 
In the present section the notation $\mathrm{P}_{\alpha}$ is no longer used for the function in (3.1.5); it will now denote the following function (cf. (3.3.5)):

$$
\text { (3.3.7) } \quad \mathrm{P}_{\alpha}(z):=\mathrm{P}(z) / \mathrm{P}(1-\alpha+\alpha z)
$$

which is always defined in a neighbourhood of zero.

Before establishing the canonical representation of the discrete self-mdec pgf's, we state an auxiliary lemma.

LEMMA 3.3.4. If $P$ is a pgf, then

$$
\lim _{x \uparrow 1}(1-x) P^{n}(x)=0 .
$$

PROOF. As $\mathrm{P}^{\prime}$ is nondecreasing on $[0,1)$, we can write for all $x \in[0,1)$

$$
0 \leq(1-x) P^{\prime}(x) \leq \int_{(x, 1)} P^{\prime}(y) d y=1-P(x),
$$

which tends to zero as $\mathrm{x} \uparrow 1$. Hence the lemma is proved.

THEOREM 3.3.5. A pgf $\mathrm{P}$, with $0<\mathrm{P}(0)<1$, is discrete self-dec iff $\mathrm{P}$ has the form

$$
\text { (3.3.8) } P(z)=\exp \left[-\mu \int_{(z, 1)} \frac{1-Q(u)}{1-u} d u\right],
$$

where $\mu>0$ and $Q$ is a pgf with $Q(0)=0$; the representation $(\mu, Q)$ is unique. Equivalently, $P$ is discrete self-dec iff $P$ is inf div and has a canonical sequence $\left\{r_{n}(1)\right\}$ (cf. theorem 1.5.3) that is nonincreasing.

PROOF. Let $P$ be discrete self-dec, $i . e$. let the function $P_{\alpha}$ defined by $(3.3 .7)$, be a pgf. For $0 \leq z<1$ we can write

$$
P(1-\alpha+\alpha z)-P(z)=(1-\alpha)(1-z) P^{\prime}(z)+o(1-\alpha) \quad(\alpha \uparrow 1) .
$$

and hence

$$
P_{\alpha}(z)=\left\{1+(1-\alpha)(1-z) P^{\prime}(z) / P(z)+o(1-\alpha)\right\}^{-1} \quad(\alpha \uparrow 1) .
$$

Let $\gamma>0$, and take $\alpha_{n}$ such that $\gamma /\left(1-\alpha_{n}\right)=n \in \mathbb{N}, i \cdot e \cdot \alpha_{n}=1-\gamma / n$. Then for all $n \in \mathbb{N} \quad\left\{p_{\alpha_{n}}(z)\right\}^{\gamma /\left(1-\alpha_{n}\right)}$ is a pgf, for which

$$
\text { (3.3.9) } \begin{aligned}
S_{\gamma}(z) & :=\lim _{n \rightarrow \infty}\left\{P_{\alpha_{n}}(z)\right\}^{\gamma /\left(1-\alpha_{n}\right)}=\lim _{n \rightarrow \infty}\left\{1+\frac{\gamma}{n}(1-z) \frac{P^{8}(z)}{P(z)}+o\left(\frac{1}{n}\right\}^{-n}=\right. \\
& =\exp \left[-\gamma(1-z) P^{\prime}(z) / P(z)\right] .
\end{aligned}
$$


Since $S_{\gamma}(z) \rightarrow 1$ as $z \uparrow 1$ (cf. lemma 3.3.4), it is seen by theorem 1.3 .5 that $s_{\gamma}$ is a pgf for all $\gamma>0$. As $\left\{s_{1}\right\}^{\gamma}=s_{\gamma}$, it follows that $s:=s_{1}$ is an inf div pgf, and hence (theorem 1.5.1) there exist $\mu>0$ and a pgf $Q$ with $Q(0)=0$ such that $S$ is compound-Poisson- $(\mu, 2)$. From (3.3.9) it is now seen that the $R_{1}$-function of $P$ is given by

$$
R_{1}(z)=P^{\prime}(z) / P(z)=\frac{-\log S(z)}{1-z}=\mu \frac{1-Q(z)}{1-z}
$$

which yields $(3.3 .8)$. Furthermore, if $Q$ is the pgf of $\left\{q_{n}\right\}_{0}^{\infty}$, from $(3.3 .10)$ it follows that the $x_{n}(1)$ 's corresponding to $P$ are given by

(3.3.11) $r_{n}(1)=\mu \sum_{k=n+1}^{\infty} q_{k} \quad\left(n \in \mathbb{N}_{0}\right) ;$

hence $r_{n}(1) \geq 0$ for all $n \in \mathbb{N}_{0}$ (so $P \in C_{1}$ ) and $r_{n}(1)$ is nonincreasing.

Conversely, let $P \in C_{1}$ with a nonincreasing canonical sequence $\left\{r_{n}(1)\right\}$, and let $\alpha \in(0,1)$. In view of the second part of theorem 1.5 .3 , for the function $\mathrm{P}_{\alpha}$ we can write

$$
\text { (3.3.12) } \quad P_{\alpha}(z)=\exp \left[-\int_{(z, 1-\alpha+\alpha z)} R_{1}(u) d u\right] .
$$

so that

$$
\frac{d}{d z} \log P_{\alpha}(z)=R_{1}(z)-\alpha R_{1}(1-\alpha+\alpha z) .
$$

This function has a power-series expansion with the following coefficients:

$$
\begin{aligned}
& r_{n}(1)-\alpha^{n+1} \sum_{k=n}^{\infty}\left(\begin{array}{l}
k \\
n
\end{array}\right)(1-\alpha)^{k-n} r_{k}(1) \geq r_{n}(1)\left\{1-\alpha^{n+1} \sum_{k=0}^{\infty}\left(\begin{array}{c}
n+k \\
k
\end{array}\right)(1-\alpha)^{k}\right\}= \\
& =r_{n}(1)\left\{1-\alpha^{n+1} \sum_{k=0}^{\infty}(-1)^{k}\left(\begin{array}{c}
-n-1 \\
k
\end{array}\right)(1-\alpha)^{k}\right\}=0,
\end{aligned}
$$

where we have used the fact that $r_{n}(1)$ is nonincreasing. It follows that $\log \left\{P_{\alpha}(z) / P_{\alpha}(0)\right\}$, and hence $P_{\alpha}$, is abs mon. Finally, $P_{\alpha}(z) \rightarrow 1$ as $z \uparrow 1$, i.e. $P_{\alpha}$ is a pgf, so $P$ is discrete self-dec.

COROLLARY 3.3.6. The factors $\mathrm{P}_{\alpha}(0<\alpha<1)$ of a discrete self-dec pgf $\mathrm{P}$ are all inf div.

COROLIARY 3.3.7. If $\left\{p_{n}\right\}_{0}^{\infty}$ is discrete self-dec wi.th $0<p_{0}<1$, then $p_{n}>0$ for all $n \in \mathbb{N}_{0}$. 
PROOF. If $p_{1}$ would vanish, then $x_{0}(1)=p_{1} / p_{0}=0$, and hence, as $r_{n}(1)$ is nonincreasing, $r_{n}(1)=0$ for all $n \in \mathbb{N}_{0}$, which is only possible if $p_{0}=1$. It follows that $p_{1}>0$, but then $p_{n}>0$ for all $n \in \mathbb{N}_{0}$ by theorem 1.5.7.

The unimodality of discrete self-dec distributions is a corollary to the following theorem (cf. corollary 1.5.5).

THEOREM 3.3.8. Let $\left\{p_{n}\right\}_{0}^{\infty}$ and $\left\{r_{n}\right\}_{0}^{\infty}$ be sequences of real numbers with $p_{0}>0$, $p_{n} \geq 0 \quad(n \in \mathbb{N}), r_{n}$ nonincreasing and such that

$$
\text { (3.3.13) } \quad(n+1) p_{n+1}=\sum_{k=0}^{n} p_{k} x_{n-k} \quad\left(n \in \mathbb{N}_{0}\right)
$$

Then $\left\{p_{n}\right\}_{0}^{\infty}$ is unimodal, i.e. $p_{n}-p_{n-1}$ changes sign at most once (put $p_{-1}=0$ ); $\left\{p_{n}\right\}_{0}^{\infty}$ is nonincreasing iff $r_{0} \leq 1$.

PROOF. Our proof is suggested by the proof of Wolfe (1971a) for self-decomposable densities on $(0, \infty)$. First we introduce the sequences $\left\{d_{n}\right\}_{0}^{\infty}$ and $\left\{\lambda_{n}\right\}_{0}^{\infty}$ by

$$
d_{n}:=p_{n}-p_{n-1}, \lambda_{n}:=r_{n}-r_{n+1} \quad\left(n \in \mathbb{N}_{0}\right)
$$

Because of the monotonicity of $\left\{r_{n}\right\}$ we have $\lambda_{n} \geq 0 \quad\left(n \in \mathbb{N}_{0}\right)$, while

(3.3.14) $r_{n}=x_{0}-\sum_{k=0}^{n-1} \lambda_{k} \quad(n \in \mathbb{N})$.

From (3.3.13) we obtain by subtraction

$$
\text { (3.3.15) }(n+1) d_{n+1}=\left(x_{0}-1\right) p_{n}-\sum_{k=0}^{n-1} \lambda_{k} p_{n-k-1} \quad\left(n \in \mathbb{N}_{0}\right),
$$

and hence $\mathrm{d}_{\mathrm{n}} \leq 0$ for $\mathrm{n} \in \mathbb{I N}$ iff $r_{0} \leq 1$. Now let $r_{0}>1$, and suppose that there exist $n_{1} \in \mathbb{N}$ and $n_{2}:=n_{1}+m$ (some $m \in \mathbb{I N}$ ) such that

$$
\text { (3.3.16) } \quad a_{1}>0, a_{2} \geq 0, \ldots, a_{n_{1}} \geq 0, a_{n_{1}+1}<0, \ldots, a_{n_{2}} \leq 0, a_{n_{2}+1}>0 .
$$

Then we have, putting $p_{n-j}=0$ if $j>n$,

$$
\begin{aligned}
& \text { (3.3.17) } \quad p_{n_{1}-j} \leq p_{n_{1}} \quad(j=1,2, \ldots, m) \\
& p_{n_{1}-j} \leq p_{n_{2}-j} \quad(j=m+1, m+2, \ldots) .
\end{aligned}
$$


From (3.3.15) and $(3.3 .16)$ it is seen that on the one hand

(3.3.18) $\left(n_{1}+1\right) d_{n_{1}+1}=\left(r_{0}-1\right) p_{n_{1}}-\sum_{k=0}^{n_{1}-1} \lambda_{k} p_{n_{1}-k-1}<0$.

and on the other hand

(3.3.19) $\left(n_{2}+1\right) d_{n_{2}+1}=\left(x_{0}-1\right) p_{n_{2}}-\sum_{k=0}^{n_{2}-1} \lambda_{k} p_{n_{2}-k-1}>0$.

From (3.3.19) it follows that

$$
\left(x_{0}-1\right) p_{n_{2}}>\sum_{k=0}^{m-1} \lambda_{k} p_{n_{2}}+\sum_{k=m}^{n_{2}-1} \lambda_{k} p_{n_{2}-k-1} .
$$

and hence, because of $(3.3 .14)$,

$$
\left(r_{m}-1\right) p_{n_{2}}>\sum_{k=m}^{n_{2}-1} \lambda_{k} p_{n_{2}-k-1}
$$

Now, using this and $(3.3 .17)$, we can estimate in the following way:

$$
\begin{aligned}
& \sum_{k=0}^{n_{1}-1} \lambda_{k} p_{n_{1}-k-1} \leq \sum_{k=0}^{m-1} \lambda_{k} p_{n_{1}}+\sum_{k=m}^{n_{2}-1} \lambda_{k} p_{n_{2}-k-1}< \\
& <\left(x_{0}-x_{m}\right) p_{n_{1}}+\left(r_{m}-1\right) p_{n_{2}}<\left(x_{0}-x_{m}\right) p_{n_{1}}+\left(x_{m}-1\right) p_{n_{1}}=\left(x_{0}-1\right) p_{n_{1}},
\end{aligned}
$$

which contradicts $(3.3 .18)$. It follows that $(3.3 .16)$ is impossible.

COROLLARY 3.3.9. A discrete self-dec distribution $\left\{p_{n}\right\}_{0}^{\infty}$ is unimodal; it is nonincreasing iff $x_{0}(1)=p_{1} / p_{0} \leq 1$. Equivalently, an inf div lattice distribution with $p_{0}>0$ is unimodal if $i t s$ canonical sequence $\left\{r_{n}(1)\right\}$ is nonincreasing; it is nonincreasing iff in addition $r_{0}(1) \leq 1$.

REMARK 3.3.10. The unimodality of discrete self-dec distributions can be used to give a slightly simpler proof of the unimodality of self-dec distributions on $[0, \infty)$. The latter distributions have a Lévy function $M$ (cf. theorem 1.4 .7$)$ that is absolutely continuous with $\mathrm{XM}^{8}(\mathrm{x})$ nonincreasing on $(0, \infty)$, i.e. (cf. corollary 1.7.4) its $k$-functions are concave on $[0, \infty)$. Now, such a $K_{0}$-function can be approximated by step functions for which the step heights form a nonincreasing sequence, and hence (cf. theorem 1.7.7) by making the lattice finer it can be seen that a self-dec distribution on $[0, \infty)$ 
is the limit of discrete self-dec distributions. This procedure amounts to a more drastic discretization than that used by wolfe (1971a).

REMARK 3.3.11. In theorem 3.3.8 the $r_{n}$ 's are not supposed to be all nonnegative, i.e. we seem to find a sufficient condition for unimodality of more general sequences than inf div lattice distributions. For nonnegative $p_{n}{ }^{\prime} s$, however, $r_{n}$ nonincreasing implies $r_{n} \geq 0\left(n \in \mathbb{N}_{0}\right)$, which can be shown as folllows.

First, let $\left\{p_{n}\right\}$ be nondecreasing. Suppose that there exists $n_{0} \in \mathbb{N}$ such that $r_{n_{0}}<0$, then for all $n>n_{0}$ we have

$$
(n+1) p_{n+1} \leq \sum_{k=0}^{n-1} r_{k} p_{n-k} \leq r_{0} n_{o} p_{n},
$$

which contradicts the fact that $\left\{p_{n}\right\}$ is nondecreasing.

If $\left\{p_{n}\right\}$ is not nondecreasing, then, as $\left\{p_{n}\right\}$ is unimodal, $\left\{p_{n}\right\}$ is bounded: $p_{n} \leq \mathbb{M}$, say, for all $n \in \mathbb{N}_{0}$. Suppose that not all $x_{n}{ }^{\prime} s$ are nonnegative, then there exist $c>0$ and $n_{0} \in \mathbb{N}$ such that $r_{n} \leq-c$ for all $n \geq n_{0}$. It follows that

$$
(n+1) p_{n+1} \leq r_{0} \sum_{k=0}^{n_{0}-1} p_{n-k}-c \sum_{k=0}^{n-n} p_{k} \cdot
$$

Now, if $\Sigma p_{n}=\infty$, then we can choose $N \in \mathbb{N}$ such that for $n$ sufficiently large

$$
(n+1) p_{n+1} \leq r_{0} n_{0} M-c N<0 ;
$$

similarly, if $\sum \mathrm{p}_{\mathrm{n}}=: \ell<\infty$, then we can choose $\varepsilon>0$ and $\delta>0$, such that for $n$ sufficiently large

$$
(n+1) p_{n+1} \leq r_{0} n_{0} \varepsilon-c(l-\delta)<0 .
$$

Thus in both cases we have obtained a contradiction to the fact that $p_{n} \geq 0$ for all $\mathrm{n} \in \mathbb{I N} \mathrm{O}^{\circ}$

The class of self-dec distributions contains a very important subclass, viz. the class of stable distributions, which can be introduced as follows (cf. Feller (1971), ch. VI): a $r v X$ is said to be (strictly) stable with exponent $\gamma$ (necessarily $\in(0,2]$ ) if 


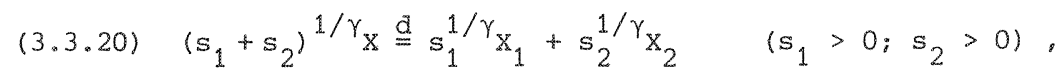

where $x_{1}$ and $x_{2}$ are independent $r v^{\prime} s$ with the same distribution as $x$. We rewrite $(3.3 .20)$ as

$$
\text { (3.3.21) } \quad \mathrm{x}=\alpha \mathrm{x}_{1}+\left(1-\alpha^{\gamma}\right)^{1 / \gamma_{\mathrm{x}}} \mathrm{x}_{2} \quad(0<\alpha<1),
$$

or in terms of che's

$$
\text { (3.3.22) } \tilde{F}(t)=\widetilde{F}(\alpha t) \tilde{F}\left((1-\alpha)^{1 / \gamma} t\right) \quad(t \in \mathbb{R} ; 0<\alpha<1) .
$$

Now, analogous to the definition of discrete self-dec distributions, we introduce discrete stable distributions by replacing $\alpha \mathrm{x}_{1}$ in $(3.3 .21)$ by $\alpha \circ \mathrm{x}_{1}$ as given by example 3.3 .1 (iii), and similarly for the other term in (3.3.21). In terms of $\mathrm{pgf}^{\mathrm{s}} \mathrm{s}$ we then obtain the following definition.

DEFINITION 3.3.12. A pgf $P$ with $0<P(0)<1$ is said to be (strictly) discrete stable with exponent $\gamma>0$ if it satisfies

$$
\text { (3.3.23) } \mathrm{P}(z)=\mathrm{P}(1-\alpha+\alpha . z) \mathrm{P}\left(1-\left(1-\alpha^{\gamma}\right)^{1 / \gamma}(1-z)\right) \quad(|z| \leq 1 ; 0<\alpha<1) .
$$

Comparing the defining relations (3.3.5) and (3.3.23), we see that, as in the continuous case, the following theorem holds.

THEOREM 3.3.13. A discrete stable distribution is discrete selfmdec, and hence unimodal.

Next we establish the canonical representation of a discrete stable distribution.

THEOREM 3.3.14. A pgf $\mathrm{P}$ with $0<\mathrm{P}(0)<1$ is discrete stable with exponent $\gamma$ iff $P$ has the form

$$
\text { (3.3.24) } P(z)=\exp \left[-\mu(1-z)^{\gamma}\right]=: 2_{\gamma}^{\mu}(z) \quad(|z| \leq 1),
$$

where $\mu>0$. The exponent $\gamma$ necessarily satisfies $0<\gamma \leq 1$.

PROOF. Let $P$ be discrete stable with exponent $\gamma$. As for $0 \leq z<1$ we can write

$$
P(1-\alpha+\alpha z)-P(z)=(1-\alpha)(1-z) P^{\prime}(z)+o(1-\alpha) \quad(\alpha \uparrow 1)
$$


it follows from $(3.3 .23)$, with $u_{\alpha}:=\left(1-\alpha^{\gamma}\right)^{1 / \gamma}$, that

$$
\begin{aligned}
\lim _{\alpha \uparrow 1} \frac{1-P\left(1-u_{\alpha}(1-z)\right)}{1-\alpha} & =\lim _{\alpha \uparrow 1} \frac{1}{P(1-\alpha+\alpha z)} \frac{P(1-\alpha+\alpha z)-P(z)}{1-\alpha}= \\
& =(1-z) P^{\prime}(z) / P(z) .
\end{aligned}
$$

or, equivalently,

$$
\text { (3.3.25) } \lim _{\alpha \uparrow 1} \frac{1-P\left(1-u_{\alpha}(1-z)\right)}{u_{\alpha}^{\gamma}}=\frac{1}{\gamma}(1-z) p^{2}(z) / P(z) \quad(0 \leq z<1) .
$$

As for $0 \leq z<1$ we have $\alpha \uparrow 1$ iff $v:=u_{\alpha}(1-z)+0$, we can rewrite (3.3.25) in the form

$$
\text { (3.3.26) } \lim _{v \downarrow 0} \frac{1-P(1-v)}{v^{\gamma}}=\frac{1}{\gamma}(1-z)^{1-\gamma} P^{\prime}(z) / P(z) \quad(0 \leq z<1) .
$$

As the left-hand side, and hence the right-hand side, of (3.3.26) is independent of $z \in[0,1)$, we get, by taking $z=0$ in $(3.3 .26)$,

$$
(3.3 .27) \quad P^{8}(z) / P(z)=\frac{P_{1}}{P_{0}}(1-z)^{\gamma-1} \quad(0 \leq z<1) .
$$

Integrating this equation and observing that the resulting function is analytic in $|z| \leq 1$, we see that $P$ has the form (3.3.24), with $\mu$ given by

(3.3.28) $\mu=\gamma^{-1} p_{1} / p_{0}=r_{0}(1) / \gamma$

Conversely, if $P=Q_{\gamma}^{\mu}$, then it is easily verified that $P$ satisfies $(3.3 .23)$, i.e. $P$ is discrete stable.

Finally, as $\mathrm{P}^{\prime}(1)>0$ (possibly infinite) unless $P(0)=1$, from (3.3.27) it is seen that $0<\gamma \leq 1$.

COROLLARY 3.3.15. A discrete stable distribution $\left\{p_{n}\right\}_{0}^{\infty}$ has a finite first moment iff its exponent $\gamma$ is equal to 1 , in which case $\left\{\mathrm{p}_{\mathrm{n}}\right\}$ is Poissonian.

REMARK 3.3.16. From $(3.3 .27)$ we see that the canonical sequence $\left\{r_{n}(1)\right\}$ of $Q_{\gamma}^{\mu}$ is given by

$$
\text { (3.3.29) } \quad x_{n}(1)=\mu \gamma(-1)^{n}\left(\begin{array}{c}
\gamma-1 \\
n
\end{array}\right)=\mu \gamma\left(\begin{array}{c}
n-\gamma \\
n
\end{array}\right) \quad\left(n \in \mathbb{N}_{0}\right) .
$$

As $r_{n}(1) \geq 0$ for all $n\left(Q_{\gamma}^{\mu}\right.$ is inf div), we see also from (3.3.29) that necessarily $0<\gamma \leq 1$. 
The discrete stable pgf's, i.e. the pgf's $Q_{\gamma}^{\mu}(0<\gamma \leq 1 ; \mu>0)$ from $(3.3 .24)$, are quite similar to the PLST's of the stable aistributions on $[0, \infty)(\mathrm{CF}$. Feller (1971), ch. XIII): a df $F$ on $[0, \infty)$ is stable with exponent $\gamma$ (necessarily $\in(0,1])$ iff $\vec{F}$ has the form

$(3.3 .30) \hat{F}(\tau)=\exp \left[-\mu \tau^{\gamma}\right]=: \hat{G}_{\gamma}^{\mu}(\tau) \quad(\tau \geq 0)$

where $\mu>0$. Rather curiously, in the discrete case the poisson distribution replaces the degenerate one (cf. corollary 3.3.15).

$A$ af $F$ on $[0, \infty)$ is said to be in the domain of attraction of $\mathrm{G}^{\mu}$ if there exist $\alpha_{n}(n \in \mathbb{N})$ such that

(3.3.31) $\lim _{n \rightarrow \infty}\left\{\hat{F}\left(\alpha_{n} \tau\right)\right\}^{n}=\hat{G}_{\gamma}^{\mu}(\tau) \quad(\tau \geq 0)$ or, if $x_{1}, x_{2} \ldots$ are independent $r v^{\prime} s$ with df $F$ and if $Y_{Y}$ has af $G_{Y}^{\mu}$

$$
\lim _{n \rightarrow \infty} \alpha_{n}\left(x_{1}+\ldots+x_{n}\right) \stackrel{d}{=} y_{\gamma} .
$$

As shown in e.g. Fellex (1971), ch. XIII, only a stable PLST appears as a limit like in the left-hand side of (3.3.31). Furthermore the $\alpha_{n}{ }^{8} \mathrm{~s}$ necessarily satisfy

$$
(3.3 .32) \quad \alpha_{n} \rightarrow n^{-1 / \gamma} \quad(n \rightarrow \infty)
$$

Now, similarly, a lattice distribution $\left\{\mathrm{p}_{\mathrm{n}}\right\}_{0}^{\infty}$ with $\mathrm{p}_{0}>0$ is said to be in the domain of discrete attraction of $Q_{\gamma}^{\mu}$ if there exist $\alpha_{n}$ ( $n \in \mathbb{N}$ ) such that

$$
\text { (3.3.33) } \lim _{n \rightarrow \infty}\left\{p\left(1-\alpha_{n}+\alpha_{n} z\right)\right\}^{n}=Q_{\gamma}^{\mu}(z) \quad(|z| \leq 1) .
$$

Taking $\alpha_{n}$ such that (3.3.32) holds, we see that $Q_{\gamma}^{\mu}$ belongs to its own domain of attraction. Furthermore we have the following property.

THEOREM 3.3.17. Every lattice distribution with finite first moment belongs to the domain of discrete attraction, of $Q_{1}^{\mu}$, 1 .e. of the poisson distribution.

PROOF. Let $\mathrm{P}$ be a pgf with $\mathrm{P}^{\prime}(1)=: \mu<\infty$, and let $z \in[0,1)$. Then for all $n \in \mathbb{N}$ there exists $\xi_{n} \in\left(1-\frac{1}{n}(1-z), 1\right)$ such that

$$
1-P\left(1-\frac{1}{n}(1-z)\right)=\frac{1}{n}(1-z) P^{8}\left(\xi_{n}\right) .
$$


Now, as $\lim _{n \rightarrow \infty} P^{\prime}\left(\xi_{n}\right)=\mu$, it follows that for $0 \leq z<1$, and hence by the continuity theorem for all $|z| \leq 1$.

$$
\lim _{n \rightarrow \infty}\left\{P\left(1-\frac{1}{n}(1-z)\right)\right\}^{n}=\lim _{n \rightarrow \infty}\left\{1-\frac{\mu}{n}(1-z)+o\left(\frac{1}{n}\right)\right\}^{n}=\exp [\mu(z-1)] .
$$

A general theory of attraction could easily be developed. The domains of discrete attraction, however, are completely determined by their continuous counterparts. In fact, for every $\gamma \in(0,1]$ and $\mu>0$ we have

$$
Q_{\gamma}^{\mu}(1-\tau)=\hat{G}_{\gamma}^{\mu}(\tau) \quad(\tau \geq 0)
$$

and as for every $\tau \geq 0$

$$
\begin{aligned}
\left\{P_{X}\left(1-\alpha_{n} \tau\right)\right\}^{n} & =\left\{E \exp \left[X \log \left(1-\alpha_{n} \tau\right)\right]\right\}^{n} \sim \\
& \sim\left\{E \exp \left[-\alpha_{n} \tau X\right]\right\}^{n}=\left\{\hat{F}_{X}\left(\alpha_{n} \tau\right)\right\}^{n} \quad(n \rightarrow \infty)
\end{aligned}
$$

where $\mathrm{X}$ is an $\mathbb{N}_{0}$-valued $\mathrm{rv}$ with $\mathrm{pgf} \mathrm{P}_{\mathrm{X}}$ and $\mathrm{df} \mathrm{F}_{\mathrm{X}^{\prime}}$ it follows that

$$
\lim _{n \rightarrow \infty}\left\{P_{X}\left(1-\alpha_{n}+\alpha_{n} z\right)\right\}^{n}=Q_{\gamma}^{\mu}(z) \Leftrightarrow \lim _{n \rightarrow \infty}\left\{\hat{F}_{X}\left(\alpha_{n} \tau\right)\right\}^{n}=\hat{G}_{\gamma}^{\mu}(\tau)
$$

i.e. an $\mathbb{N}_{0}$-valued $x \mathrm{x} x$ is in the domain of discrete attraction of $Q_{\gamma}^{\mu}$ iff it is in the domain of attraction of $G_{\gamma}^{\mu}$.

\section{4. a-decomposable(1) lattice distributions}

In this section we briefly consider pgf'S $P$ that have $1-\alpha+\alpha P(z)$ as a factor (cf. example 3.3.1(ii)). Proceeding as in sections 1 and 2 for $\alpha$-dec pgf's (which have $P(\alpha z) / P(\alpha)$ as a factor), we obtain properties that are similar to those of the $\alpha$-dec pgf's. The main purpose is to give an analogue of theorem 3.2 .4 for $C_{0}$.

DEFINITION 3.4.1. For $0<\alpha<1$ a pgf $\mathrm{p}$ with $\mathrm{P}(0)>0$ is said to be $\alpha$-decomposable(1) $(\alpha-\operatorname{dec}(1))$ if there exists a pgf $\mathrm{P}_{\alpha}$ such that

$$
\text { (3.4.1) } \quad P(z)=\{1-\alpha+\alpha P(z)\}_{\alpha}(z) \quad(|z| \leq 1) .
$$

$P$ is called totalzy-decomposable(1) if $P$ is $\alpha$-dec(1) for all $\alpha \in(0,1)$.

For an arbitrary pgf $\mathrm{P}$ with $\mathrm{P}(0)^{\circ}>0$ and for $\alpha \in(0,1)$ we denote by $\mathrm{P}_{\alpha}$ the following function (cf. $(3,4.1))$ : 
(3.4.2) $\quad P_{\alpha}(z):=P(z) /\{1-\alpha+\alpha P(z)\}$,

which is always defined in some neighbourhood of zero. Obviously, we have the following criterion (cf. lemma 1.3.4) and representation lemma (solve $(3.4 .1)$ for $P)$.

LEMMA 3.4.2. For $0<\alpha<1$ a pgf $\mathrm{P}$ with $\mathrm{P}(0)>0$ is $\alpha-\operatorname{dec}(1)$ iff $\mathrm{P} \alpha$ is abs mon.

LEMMA 3.4.3. If $0<\alpha<1$ and if $P$ is a pgf with $P(0)>0$, then $P$ is $\alpha$-dec(1) iff $P$ has the form

$(3.4 .3)$

$$
P(z)=\frac{1-\alpha}{1-\alpha Q(z)} Q(z) \quad(|z| \leq 1),
$$

where $Q$ is a pgf. The representation is unique: $Q=\mathrm{P}_{\alpha}$.

Thus, an $\alpha$-dec(1) pgf $\mathrm{P}$ has a compound geometric factor. It turns out that the factor $\mathrm{P}_{\alpha}$ of $\mathrm{P}$ is in $\mathrm{C}_{0}$ iff $\mathrm{P} \in \mathrm{C}_{0}$. We state this in the following lemra, which is easily verified by using theorem 2.4 .9 (ii) and the following relation between the $R_{0}$-functions $R_{0}$ and $R_{0}^{(\alpha)}$ of $P$ and $P_{\alpha}$, respectively:

$$
R_{0}(z)=\left\{1+\frac{\alpha}{1-\alpha} P(0)\right\} R_{0}^{(\alpha)}(z)
$$

LEMMA 3.4.4. If $P$ is a pgf with $P(0)>0$, then $P \in C_{0}$ iff for some, and then for all, $\alpha \in(0,1)$ is $\alpha$-dec(1) with factor $\mathrm{P}_{\alpha} \in \mathcal{C}_{0}$.

By expressing $P_{\beta}$ in $P_{\alpha}$ one obtains the following implication:

(3.4.4) $[\mathrm{P} \alpha-\operatorname{dec}(1), 0<\beta<\alpha<1] \Rightarrow P \beta-\operatorname{dec}(1)$.

It follows that the classes of $\alpha$-dec(1) pgf's are decreasing in $\alpha$. Now the limiting class $(\alpha \uparrow 1)$ turns out to be $C_{0}$, i.e. we have the following analogue of theorem 3.2 .4 for $\mathrm{C}_{0}$.

THEOREM 3.4.5. A pgf $\mathrm{P}$ with $\mathrm{P}(0)>0$ is in $\mathrm{C}_{0}$ iff $\mathrm{P}$ is tot-dec(1).

PROOF. In view of lemma 3.4 .4 we only need show that a tot-dec(1) pgf is in $C_{0}$. So let $P$ be tot-dec(1), i.e. let $P_{\alpha}$ be a pgf for all $\alpha \in(0,1)$. Rewrite $\mathrm{P}_{\alpha}$ as follows

$$
P_{\alpha}(z)=\left\{1+(1-\alpha)\left[P(z)^{-1}-1\right]\right\}^{-1}
$$


let $\gamma>0$ and take $\alpha_{n}=1-\gamma / n(n \in \mathbb{N})$. Then it follows that $\left\{p_{\alpha_{n}}(z)\right\}^{\gamma /\left(1-\alpha_{n}\right)}$ is a pgf $(n \in \mathbb{N})$, which satisfies

$$
\begin{aligned}
S_{\gamma}(z) & :=\lim _{n \rightarrow \infty}\left\{p_{\alpha_{n}}(z)\right\}^{\gamma /\left(1-\alpha_{n}\right)}=\lim _{n \rightarrow \infty}\left\{1+\frac{\gamma}{n}\left[p(z)^{-1}-1\right]\right\}^{-n}= \\
& =\exp \left[-\gamma\left(P(z)^{-1}-1\right)\right] .
\end{aligned}
$$

Since $S_{\gamma}(z) \rightarrow 1$ as $z \uparrow 1$, by theorem 1.3 .5 it is seen that $S_{\gamma}$ is a pgf for all $\gamma>0$, and hence

$$
S_{1}(z)=\exp \left[1-P(z)^{-1}\right]
$$

is an inf div pgf. From theorem 2.4.8(ii) it now follows that $P \in C_{0}$.

COROLIARY 3.4.6. The factors $P_{\alpha}(0<\alpha<1)$ of a tot-dec(1) pgf are all in Co:

Finally, we note that (cf. sections 1 and 2 ) we might consider $\alpha-f a c t(1)$ $\operatorname{pgf}^{\prime} s, i . e . \operatorname{pgf}$ 's $P$ of the form $\{1-\alpha+\alpha Q(z)\} Q(z)$, but we shall not do so here. 



\section{CHAPTER 4}

THE CLASSES $C_{\alpha}$ IN RELATION TO RENEWAL THEORY

In chapter 2 we introduced the classes $C_{\alpha}(0 \leq \alpha \leq 1)$. Most of their properties, given there, were most easily formulated in terms of pgf's. Now, in the present chapter, we want to dexive properties of the probabilities themselves; in particular, we consider inequalities, asymptotic behaviour and closure properties.

The recurrence relations by means of which $C_{0}$ can be characterized (cf. com rollary 1.5.9), are very similar to those defining the somcalled renewal sequences (class $R_{0}$ ). This class $R_{0}$ has many well-known propexties, which are often easily obtained by a probabilistic intexpretation (cf. Feller (1968), ch. XIII and Kingman (1972), ch. 1). Now, using the relation between $C_{0}$ and $R_{0}$, we obtain similar properties for $C_{0}$ (section 1). In section 2. we consider the case $0<\alpha<1$. We introduce classes $R_{\alpha}$ of generalized renewal sequences that are related to the $C_{\alpha}{ }^{8} \mathrm{~s}$ in the same way as $R_{0}$ is related to $C_{0}$, and we investigate to what extent the properties of $R_{0}$ can be extended to the $R_{\alpha}{ }^{\prime} s$. Specifically, we look for a probabilistic interpretation of the sequences in $R_{\alpha}$. We do find interpretations, but these axe rather complicated and yield only few results. Finally, in section 3 we briefly consider an extension $R_{1}$ of $C_{1}$.

4.1. The class $C_{0}$ and discrete-time renewal theory The class $C_{0}$ of compound geometric lattice distributions $\left\{p_{n}\right\}_{0}^{\infty}$ can be cham racterized by means of the recurrence relations

(4.1.1a) $p_{n+1}=\sum_{k=0}^{n} p_{k} x_{n-k}(0) \quad\left(n \in \mathbb{N}_{0}\right)$

where the sequence $\left\{r_{n}(0)\right\}_{0}^{\infty}$ satisfies

$(4.1 .1 b) \quad r_{n}(0) \geq 0\left(n \in \mathbb{N}_{0}\right), r(0):=\sum_{n=0}^{\infty} x_{n}(0)<1$.

Now, the recurrence relations $(4.1 .1$ a) are very similar to those defining the so-called renewal sequences (cf. Kingman (1972), ch. 1): a sequence $\left\{u_{n}\right\}_{0}^{\infty}$, with $u_{0}=1$, is said to be a renewal sequence (or of class $R_{0}$ ) if it satisfies 
(4.1.2a) $u_{n}=\sum_{k=1}^{n} f_{k} u_{n-k} \quad(n \in \mathbb{N})$

where the sequence $\left\{f_{n}\right\}_{1}^{\infty}$ is such that

$(4.1 .2 b) \quad f_{n} \geq 0 \quad(n \in \mathbb{N}), f:=\sum_{n=1}^{\infty} f_{n} \leq 1:$

in this case $\left\{u_{n}\right\}$ is called the renewal sequence associated with $\left\{f_{n}\right\}_{1}^{\infty}$. In fact, we have the following relation between $C_{0}$ and $R_{0}$.

LEMMA 4.1 .1$.

(i) If $\left\{p_{n}\right\} \in C_{0}$ and if $u_{n}:=p_{n} / p_{0}\left(n \in \mathbb{N}_{0}\right)$, then $\left\{u_{n}\right\} \in R$ and $\left\{u_{n}\right\}$ is associated with $\left\{f_{n}\right\}_{1}^{\infty}$ given by $f_{n}=r_{n-1}(0) \quad(n \in \mathbb{N})$.

(ii) If $\left\{u_{n}\right\} \in R_{0}$, associated with $\left\{\tilde{f}_{n}\right\}$, then

a) If $f<1$ and $p_{n}:=(1-f) u_{n}\left(n \in \mathbb{N}_{0}\right)$, then $\left\{p_{n}\right\} \in C_{0}$ with $r_{n}(0)=$ $=f_{n+1}\left(n \in \mathbb{N}_{0}\right):$

b) If $f=1$ and $p_{n}:=\gamma^{n} u_{n} / v(\gamma)$, where $\gamma \in(0,1)$, then $\left\{p_{n}\right\} \in C_{0}$ wi.th $r_{n}(0)=\gamma^{n+1} f_{n+1}\left(n \in \mathbb{N}_{0}\right)$.

PROOF.

(i) immediately follows by comparing (4.1.1a) with $(4.1 .2 a)$, and $(4.1 .1 \mathrm{~b})$ with $(4.1 .2 \mathrm{~b})$.

(ii) Let $\left\{u_{n}\right\} \in R_{0}$, associated with $\left\{f_{n}\right\}$. If $f<1$, then, as we shall see in theorem $4.1 .2(i), \sum_{n=0}^{\infty} u_{n}=(1-f)^{-1}$ is finite. Hence $\left(c f\right.$. (i)), if $p_{n}:=$ $:=(1-f) u_{n}$, then $\left\{p_{n}\right\}^{n=0} \in C_{0}$. If $f=1$, then $\sum_{n=0}^{\infty} u_{n}=\infty$, but $U(\gamma)=\sum_{n=0}^{\infty} u_{n} \gamma^{n}<\infty$ for all $\gamma \in(0,1)$ (c.f. lemma 4.1.3). It follows that $\left\{p_{n}\right\}^{\infty}$, with $p_{n}:=$ $:=\gamma^{n} u_{n} / U(\gamma)$, is a probability distribution that satisfies (4.1.1a) with $r_{n}(0)=\gamma^{n+1} f_{n+1} \geq 0$. Hence $\left\{p_{n}\right\} \in C_{0}$.

The recurrence relations $(4.1 .2 a)$ have been studied by several authors, e.g. by Kaluza (1928), De Bruijn \& Erdös (1951), Lampexti (1958), Kendal1 (1967), Feller (1968) and kingman (1972). The main properties of these relations will be given in the remainder of this section. Using lemma 4.1 .1 we can transfer most of these properties to $C_{0}$, but we shall do so only if this is of special interest.

In the next section we want to investigate to what extent the properties that can be obtained for $C_{0}$, can be extended to $C_{\alpha}(0<a<1)$. As, to this 
end, the sequences in $C_{\alpha}$ will be related to the so-called delayed renewal. sequences, we consider recurrence relations that are slightly more general than those in $(4.1 .2 a)$.

Our starting point is the theory of recurrent events of Feller (1968), ch. XIII, but we prefer a formulation similar to the one usual for renewal theory in continuous time (cf. Feller (1971), ch. XI). Let us introduce two sequences $\left\{b_{n}\right\}_{0}^{\infty}$ and $\left\{f_{n}\right\}_{1}^{\infty}$ satisfying

$(4.1 .3) \quad b_{n} \geq 0 \quad\left(n \in \mathbb{N}_{0}\right), 0<b:=\sum_{n=0}^{\infty} b_{n}<\infty, f_{n} \geq 0 \quad(n \in \mathbb{N}), 0<f:=\sum_{n=1}^{\infty} f_{n}<\infty$. Then the (discrete) renewal equation associated with $\left\{b_{n}\right\}$ and $\left\{f_{n}\right\}$, is defined by

(4.1.4) $\quad v_{n}=b_{n}+\sum_{k=1}^{n} f_{k} v_{n-k} \quad\left(n \in \mathbb{N}_{0}\right)$

It is called pure if $b_{n}=\delta_{0, n}$ and delayed otherwise. Clearly, the renewal equation has a unique solution $\left\{\mathrm{v}_{\mathrm{n}}\right\}^{\infty}$, and its gf $\mathrm{V}$ satisfies

(4.1.5) $\quad \mathrm{V}(\mathrm{z})=\mathrm{B}(\mathrm{z}) /\{1-\mathrm{F}(\mathrm{z})\}$,

or, if $\left\{u_{n}\right\}_{0}^{\infty}$ is the solution of the pure renewal equation associated with $\left\{f_{n}\right\}$,

$(4.1 .6) \quad V(z)=B(z) U(z)$.

The period $d$ of a sequence $\left\{a_{n}\right\}_{0}^{\infty}$ of real numbers (not all $a_{n}(n \in \mathbb{N}$ ) zero) is defined as follows:

$(4.1 .7) \quad a:=\operatorname{gcd}\left\{n \in \mathbb{N} \mid a_{n} \neq 0\right\}$.

Here the value of $a_{0}$ is not relevant; the period of a seguence $\left\{a_{n}\right\}_{1}^{\infty}$ is also defined by $(4.1 .7)$. Of course, $(4.1 .7)$ is equivalent to the definition of Feller (1968), who defines the period of $\left\{a_{n}\right\}$ by

$$
\mathrm{d}:=\max \left\{\mathrm{k} \in \mathbb{N} \mid \forall_{\mathrm{n} \in \mathbb{N}}\left[\mathrm{a}_{\mathrm{n}} \% 0 \Rightarrow \mathrm{k} \mid \mathrm{n}\right]\right\} .
$$

If $d=1$, then the sequence $\left\{a_{n}\right\}$ is called aperiodic. Now we are ready to state the following basic result, which is known as the renewal theorem (cf. Fellex (1968), ch. XIII)。

THEOREM 4.1.2. Suppose (4.1.3) and that $\left\{f_{n}\right\}_{1}^{\infty}$ is aperiodic. Then the solution $\left\{v_{n}\right\}_{0}^{\infty}$ of the renewal equation associated with $\left\{b_{n}\right\}$ and $\left\{f_{n}\right\}$ has the 
following properties:

(i) $\mathrm{E}<1$ iff $\mathrm{v}:=\sum_{\mathrm{n}=0}^{\infty} \mathrm{v}_{\mathrm{n}}<\infty$, in which case $\mathrm{v}=\mathrm{b} /(1-\mathrm{f})$;

(ii) If $f=1$, then $v_{\infty}:=\lim _{n \rightarrow \infty} v_{n}$ exists, and $v_{\infty}=b / \mu$ with $\mu:=\sum_{n=1}^{\infty} n f_{n}$ $(\leq \infty)$;

(iii) If $f>1$, then $\lim _{n \rightarrow \infty} x_{0}^{n} v_{n}=B\left(x_{0}\right) /\left\{x F^{\prime}\left(x_{0}\right)\right\}$, where $x_{0} \in(0,1)$ is such that $F\left(x_{0}\right)=1$.

We shall frequently use the following characterization of the bounded solutions of the renewal equation.

LEMMA 4.1.3. The solution $\left\{v_{n}\right\}_{0}^{\infty}$ of the renewal equation (4.1.4) is bounded iff $f \leq 1$, in which case $v_{n} \leq b$ for all $n \in \mathbb{N}_{0}$.

PROOF. Let $\left\{\mathrm{v}_{\mathrm{n}}\right\}$ be bounded. Then its gf $\mathrm{v}$ exists, and does not vanish, on $(0,1)$. Hence by $(4.1 .5)$ we have

$$
F(x)=1-B(x) / V(x) \leq 1 \quad(0<x<1),
$$

from which, letting $x \uparrow 1$, we see that $f=F(1) \leq 1$.

Conversely, let $f \leq 1$. Considering first the solution $\left\{u_{n}\right\}_{0}^{\infty}$ of the pure renewal equation associated with $\left\{f_{n}\right\}$, from (4.1.4), using mathematical induction, we see that $u_{n} \leq 1$ for all $n \in \mathbb{N}_{0}$. Now, equation the coefficients of $z^{n}$ in $(4.1 .6)$, we obtain

$$
v_{n}=\sum_{k=0}^{n} b_{k} u_{n-k} \leq \sum_{k=0}^{n} b_{k} \leq b
$$

and the lemma is proved.

We are particularly interested in certain bounded solutions of (4.1.4), which frequently occur in probability theory, viz. the delayed renewal sequences, as they will provide a probabilistic interpretation of the sequences in $C_{\alpha}$. If $\left\{b_{n}\right\}$ and $\left\{f_{n}\right\}$ are sequences satisfying (4.1.3) with $b \leq 1$ and $f \leq 1$, then the solution $\left\{v_{n}\right\}_{0}^{\infty}$ of the renewal equation associated with $\left\{b_{n}\right\}$ and $\left\{f_{n}\right\}$ is called the delayed renewal sequence associated with $\left\{b_{n}\right\}$ and $\left\{f_{n}\right\}$. The set of such sequences is denoted by $R$. We note that a renewal sequence (cf. the beginning of this section) can be considered as a delayed renewal sequence for which $b_{n}=\delta_{0, n}$. Hence we have

$$
\text { (4.1.8) } R_{0} \subset R \text {, }
$$


and in order to distinguish the $R_{0}$--sequences from those in $R \backslash R_{0}$ we occasionally call them pure renewal sequences.

The (delayed) renewal sequences owe their name to their interpretation in renewal processes. For ease of reference we first give the definition of such processes for discrete time.

DEFINITION 4.1.4. Let $\left\{b_{n}\right\}_{0}^{\infty}$ and $\left\{f_{n}\right\}_{1}^{\infty}$ be two sequences satisfying (4.1.3) with $b \leq 1$ and $f \leq 1$. If $\mathbb{T}_{0}, \mathbb{T}_{1}, T_{2}, \ldots$ are mutually independent, $\mathbb{N}_{0}$-valued (possibly defective) $r v^{i} s$ such that $\left\{b_{n}\right\}$ is the distribution of $T_{0}$ and $\left\{f_{n}\right\}$ that of $T_{k}$ for $k \in \mathbb{N}$, then the sequence $\left\{s_{n}\right\}^{\infty}$ " defined by

$$
S_{n}=\sum_{k=0}^{n} T_{k} \quad\left(n \in \mathbb{N}_{0}\right)
$$

is called the (discrete-time) renewal process associated with $\left\{b_{n}\right\}$ and $\left\{f_{n}\right\}$. The $\mathrm{rv} \mathrm{S}_{\mathrm{n}}$ is called the $\mathrm{n}$-th renewal epoch, and $\mathrm{T}_{\mathrm{k}}$ the $\mathrm{k}$-th life time. If $f=1$, then the mean recurrence time $\mu$ is defined by

$$
\mu:=\mathrm{ET}_{1}=\sum_{n=1}^{\infty} \mathrm{nf} \mathrm{n}_{n} \quad(\leq \infty) .
$$

Finally, $\left\{\mathrm{s}_{\mathrm{n}}\right\}$ is called pure if $\mathrm{b}_{\mathrm{n}}=\delta_{0, \mathrm{n}}$ (delayed otherwise), and persistent if $b=f=1$ (transient otherwise).

THEOREM 4.1.5. A sequence $\left\{\mathrm{v}_{\mathrm{n}}\right\}_{0}^{\infty}$ is in $R$ iff there exists a (discrete-time) renewal process $\left\{\mathrm{s}_{\mathrm{n}}\right\}_{0}^{\infty}$ such that

(4.1.9) $\quad v_{n}=P\left(\exists_{k \in \mathbb{N}_{0}} s_{k}=n\right) \quad\left(n \in \mathbb{N}_{0}\right)$

If $\left\{v_{n}\right\}$ is associated with $\left\{b_{n}\right\}$ and $\left\{f_{n}\right\}$, then $\left\{s_{n}\right\}$ is associated with the same sequences, and conversely. Finally, $\left\{v_{n}\right\}$ is pure $\left(\in R_{0}\right)$ iff $\left\{s_{n}\right\}$ is pure.

In addition to the interpretation of $\left\{v_{n}\right\} \in R$, just given, there exists another one in texms of a Markov chain. A (discrete-time) Markov chain with stationary transition probabilities is a sequence $\left\{\mathrm{x}_{n}\right\}_{0}^{\infty}$ of $x v^{\prime} s$, taking values in a countable state space $S$ and satisfying the Markov property

$$
P\left(x_{n}=j \mid x_{0}, x_{1}, \ldots, x_{n-1}^{\prime}\right)=P\left(x_{n}=j \mid x_{n-1}\right) \quad(n \in \mathbb{N} ; j \in s),
$$


such that the transition probabilities

$$
p_{i j}:=P\left(x_{n}=j \mid x_{n-1}=i\right) \quad(i, j \in s)
$$

do not depend on $\mathrm{n}$. In fact, we have the following characterization of (delayed) renewal sequences (cf. Kingman (1972)).

THEOREM 4.1 .6 .

(i) A sequence $\left\{u_{n}\right\}_{0}^{\infty}$ is in $R_{0}$ iff there exist a Maxkov chain $\left\{x_{n}\right\}_{0}^{\infty}$ and a state $j \in S$ such that

$(4.1 .10) \quad u_{n}=P\left(x_{n}=j \mid x_{0}=j\right) \quad\left(n \in \mathbb{N}_{0}\right)$.

The sequence $\left\{f_{n}\right\},\left\{u_{n}\right\}$ is associated with, is then the recurrence time distribution of $j \in S$ :

$(4.1 .11) \quad f_{n}=p\left(x_{1} \neq j, \ldots, x_{n-1} \neq j, x_{n}=j \mid x_{0}=j\right) \quad(n \in \mathbb{N})$.

(ii) A sequence $\left\{v_{n}\right\}_{0}^{\infty}$ is in $R \backslash R_{0}$ iff there exist a Markov chain $\left\{x_{n}\right\}_{0}^{\infty}$ and two distinct states $i, j \in S$ such that

$(4.1 .12) \quad v_{n}=P\left(x_{n+1}=j \mid x_{0}=i\right) \quad\left(n \in \mathbb{N}_{0}\right)$.

The sequences $\left\{b_{n}\right\}$ and $\left\{f_{n}\right\},\left\{v_{n}\right\}$ is associated with, then satisfy (4.1.11) and

$(4.1 .13) \quad b_{n}=p\left(x_{1} \neq j, \ldots, x_{n} \neq j, x_{n+1}=j \mid x_{0}=i\right) \quad\left(n \in \mathbb{N}_{0}\right)$.

These interpretations can be used to obtain the following inequalities and closure properties for $R_{0}$ (cf. Kingman (1972)). An analytic proof of (i) in a slightly less general situation has been given by De Bruijn \& Erdôs (1951), but for (ii) and (iii) such proofs seem nonexistent.

THEOREM 4.1.7.

(i) If $\left\{u_{n}\right\} \in R_{0^{\prime}}$ then $u_{n} u_{k} \leq u_{n+k} \leq u_{n} u_{k}+1-u_{n}$ for all $n, k \in \mathbb{N}_{0}$.

(ii) If $\left\{u_{n}\right\} \in R_{0}$ and $k \in \mathbb{N}$, then $\left\{u_{k n}\right\}_{n=0}^{\infty} \in R_{0}$.

(iii) If $\left\{u_{n}\right\} \in R_{0}$ and $\left\{v_{n}\right\} \in R_{0}$, then also $\left\{u_{n} v_{n}\right\} \in R_{0}$.

By lemma 4.1.1 we successively obtain from the preceding theorem the following results for $C_{0}$. Only part (i) of the first corollary immediately follows from (4.1.1a) by mathematical induction (cf. lemma 4.1.3): 


$$
p_{n+1}=\sum_{k=0}^{n} r_{k}(0) p_{n-k} \leq p_{0} \sum_{k=0}^{n} r_{k}(0) \leq p_{0} \sum_{k=0}^{\infty} r_{k}(0)<p_{0} .
$$

COROLLARY 4.1.8. The following inequalities hold for $\left\{p_{n}\right\} \in C_{0}$ :

(i) $\mathrm{p}_{\mathrm{n}}<\mathrm{p}_{\mathrm{O}}$ for all $\mathrm{n} \in \mathbb{I}$;

(ii) $p_{n} p_{k} \leq p_{0} p_{n+k} \leq p_{n} p_{k}+p_{0}\left(p_{0}-p_{n}\right)$ for all $n, k \in \mathbb{N}_{0}$;

(iii) $p_{0}\left(p_{1} / p_{0}\right)^{n} \leq p_{n} \leq p_{0}-\left(p_{0}-p_{1}\right)\left(p_{1} / p_{0}\right)^{n-1}$ for all $n \in \mathbb{N}$.

COROLLARY 4.1.9. If $\left\{\mathrm{p}_{\mathrm{n}}\right\} \in \mathrm{C}_{\mathrm{o}}$ and $\mathrm{k} \in \mathbb{N}$, then $\left\{\mathrm{c}_{\mathrm{k}} \mathrm{p}_{\mathrm{kn}}\right\}_{\mathrm{n}=0}^{\infty} \in \mathrm{C}_{\mathrm{o}}$, where $\mathrm{c}_{\mathrm{k}}$ is a suitable norming constant. In particular, if $\mathrm{k}=2$, then in terms of pgf's (4.1.14) $\left\{\mathrm{P}\left(\mathrm{z}^{\frac{1}{2}}\right)+\mathrm{P}\left(-\mathrm{z}^{\frac{1}{2}}\right)\right\} /(1+\mathrm{P}(-1)) \in \mathrm{C}_{0}$.

COROLLARY 4.1.10. If $\left\{p_{n}\right\} \in C_{0}$ and $\left\{q_{n}\right\} \in C_{0}$, then also $\left\{\mathrm{cp}_{n} q_{n}\right\} \in C_{0}$ (c is a norming constant).

In view of a result of Lamperti (1958), theorem 4.1.7(iii), which also holds

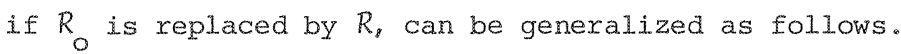

THEOREM 4.1.11. If $\left\{u_{n}\right\} \in R_{0}$ and $\left\{v_{n}\right\} \in R_{0}$, then $\left\{w_{n}\right\} \in R_{0}$ for all $\alpha \geq 0$, $\beta \geq 0, \gamma \geq 0$ (not all zero), where $\mathrm{w}_{\mathrm{n}}$ is defined by

$$
(4.1 .15) \quad w_{n}:=(\alpha+\beta+\gamma)^{-n} \sum_{i+j+k=n} \frac{n !}{i ! j ! k !} \alpha^{\dot{j}} \beta^{j} \gamma^{k} u_{i+j} v_{i+k} \quad\left(n \in \mathbb{N}_{0}\right) .
$$

PROOF. According to the result of Lamperti, $\left\{(\alpha+\beta+\gamma)^{n} w_{n}\right\}$ is a solution of the pure renewal equation. It easily follows that then also $\left\{w_{n}\right\}$ is a solution of the pure renewal equation, and as $u_{n} \leq 1$ and $v_{n} \leq 1$ (lemma 4.1.3), we see that $w_{n} \leq 1$. Applying lemma 4.1 .3 in the reverse direction, we conclude that $\left\{w_{n}\right\} \in R_{0}$.

REMARK 4.1.12. If $\alpha \neq 0$ or $\beta \neq 0$ and if $\sum u_{n}<\infty$, then $\left\{w_{n}\right\}$, multiplied with a suitable constant, is a lattice distribution in $C_{0}$ :

$$
\begin{aligned}
w_{n} & =(\alpha+\beta+\gamma)^{-n} \sum_{\ell=0}^{n}\left(\begin{array}{l}
n \\
\ell
\end{array}\right) u_{\ell} \gamma^{n-\ell} \sum_{j=0}^{\ell}\left(\begin{array}{l}
\ell \\
j
\end{array}\right) \alpha^{j} \beta^{\ell-j} v_{n-\ell+j} \leq \\
& \leq(\alpha+\beta+\gamma)^{-n} \sum_{\ell=0}^{n}\left(\begin{array}{l}
n \\
\ell
\end{array}\right) u_{\ell}(\alpha+\beta)^{\ell} \gamma^{n-\ell},
\end{aligned}
$$


and hence

$$
\begin{aligned}
\sum_{n=0}^{\infty} w_{n} & \leq \sum_{\ell=0}^{\infty} u_{\ell}(\alpha+\beta)^{\ell} \sum_{n=\ell}^{\infty}\left(\begin{array}{l}
n \\
\ell
\end{array}\right) \gamma^{n-\ell}(\alpha+\beta+\gamma)^{-n}= \\
& =\sum_{\ell=0}^{\infty} u_{\ell}(\alpha+\beta)^{\ell} \sum_{m=0}^{\infty}\left(_{m}^{-\ell-1}\right)(-\gamma)^{m}(\alpha+\beta+\gamma)^{-\ell-m}= \\
& =\sum_{\ell=0}^{\infty} u_{\ell}(\alpha+\beta)^{\ell}\left(1-\frac{\gamma}{\alpha+\beta+\gamma}\right)^{-\ell-1}(\alpha+\beta+\gamma)^{-\ell}=\frac{\alpha+\beta+\gamma}{\alpha+\beta} \sum_{\ell=0}^{\infty} u_{\ell} .
\end{aligned}
$$

of course, by lemma 4.1 .1 we can also obtain properties of $R_{0}$ from known properties of $C_{0}$. We give one example on mixtures of renewal sequences; the result seems to be new, but in fact it is a special case of theorem 4.1 .7 (iij) (take $v_{n}=p \delta_{0, n}+(1-p)$ there; then $\left\{v_{n}\right\} \in R_{0}$, as it is associated with $\left\{f_{n}\right\}$, given by $\left.f_{n}=(1-p) p^{n-1}\right)$.

THEOREM 4.1.13. If $\left\{u_{n}\right\} \in R_{0}$ and $0<p<1$, then also $\left\{w_{n}\right\} \in R_{0}$, where $(4.1 .16) \quad \mathrm{w}_{\mathrm{n}}:=\mathrm{p} \delta_{0, \mathrm{n}}+(1-\mathrm{p}) \mathrm{u}_{\mathrm{n}} \quad\left(\mathrm{n} \in \mathbb{N}_{\mathrm{O}}\right)$

PROOF. Let $\left\{u_{n}\right\} \in R_{0}$ and $0<p<1$. Then for $\left\{w_{n}\right\}$, defined by $(4.1 .16)$, we have $w_{0}=1$ and $0 \leq w_{n} \leq 1(n \in \mathbb{N})$, and its $g f w$ satisfies

$$
W(z)=p+(1-p) U(z) \quad(|z|<1) .
$$

Choose $\gamma \in(0,1)$, and define the lattice distributions $\left\{p_{n}\right\}$ and $\left\{q_{n}\right\}$ by

$$
p_{n}=\gamma^{n} u_{n} / U(\gamma), q_{n}=\gamma^{n} w_{n} / W(\gamma) \quad\left(n \in \mathbb{N}_{0}\right)
$$

Then, considering theix pgf's, we have

$$
Q(z)=W(\gamma z) / W(\gamma)=\frac{p+(1-p) U(\gamma z)}{p+(1-p) U(\gamma)}=p_{1}+\left(1-p_{1}\right) P(z)
$$

where $p_{1}:=p /\{p+(1-p) U(\gamma)\} \in(0,1)$. Since $\left\{p_{n}\right\} \in C_{0}$ (Lemma $\left.4.1 .1(i i)\right)$, from theorem 2.4.9(iii) it now follows that also $\left\{q_{n}\right\} \in C_{0^{\prime}}$ and hence, by lemma 4.1.1(i), $\left\{\gamma^{n} w_{n}\right\} \in R_{0}$. But then $\left\{w_{n}\right\}$ satisfies the pure renewal equation, and as $w_{n} \leq 1$, it follows by lemma 4.1 .3 that $\left\{w_{n}\right\} \in R_{0}$.

Next we consider the asymptotic behaviour of a renewal sequence. Of course, the strong result in this area has been already given in the renewal theorem (theorem 4.1.2), of which parts (i) and (ii) can be applied. If, however, 
$u_{\infty}:=\lim _{n \rightarrow \infty} u_{n}=0$, one can ask how fast $u_{n}$ tends to zero. The following theorem (cE. Kingman (1972)) gives an answer to this question.

THEOREM 4.1.14. Let $\left\{u_{n}\right\} \in R_{0}$ be associated with $\left\{f_{n}\right\}_{1}^{\infty}$. Then the period d of $\left\{u_{n}\right\}_{0}^{\infty}$ is equal to that of $\left\{f_{n}\right\}_{1}^{\infty}$, and

$(4.1 .17) \#\left(\left\{n \in \mathbb{N} \mid u_{n d}=0\right\}\right)<\infty$.

Furthermore $\theta:=\lim _{n \rightarrow \infty} u_{n d}^{1 /(n d)}$ exists in $(0,1]$, and

$(4.1 .18) \quad u_{n} \leq \theta^{n} \quad\left(n \in \mathbb{N}_{0}\right)$

COROLLARY 4.1.15. If $\left\{u_{n}\right\} \in R_{0}$, then the radius of convergence $\rho$ of the $g$ f $u$ of $\left\{u_{n}\right\}_{0}^{\infty}$ is Einite. Furthermore, if $v_{n}:=\rho u_{n}\left(n \in \mathbb{N}_{0}\right)$, then $\left\{v_{n}\right\}_{0}^{\infty} \in R_{0}$.

PROOF. We have

$$
\rho=\left\{\limsup _{n \rightarrow \infty} u_{n}^{1 / n}\right\}^{-1}
$$

Since in view of theorem 4.1 .14

$$
\limsup _{n \rightarrow \infty} u_{n}^{1 / n}=\limsup _{n \rightarrow \infty} u_{n d}^{1 /(n d)}=\lim _{n \rightarrow \infty} u_{n d}^{1 /(n d)}=\theta>0
$$

it follows that

$(4.1 .19) \quad \rho=\theta^{-1}<\infty$.

Now, if $v_{n}:=\rho u_{n}$, from $(4.1 .18)$ it is seen that $v_{n} \leq 1$ ( $n \in \mathbb{N}_{0}$, and, as $\left\{v_{n}\right\}$ obviously satisfies the renewal equation, we conclude by lemma 4.1 .3 that $\left\{v_{n}\right\} \in R_{0}$

Now, applying the renewal theorem to the sequence $\left\{v_{n}\right\}$, thus obtained, we can improve on the second part of theorem 4.1 .14 in some sense. We state the results in the following theorem. They are interesting only if $\rho>1$, which implies that $\sum_{n=0}^{\infty} u_{n}<\infty, i . e$. In fact we considex $C_{0}$.

THEOREM 4.1.16. Let $\left\{u_{n}\right\} \in R_{0}$ associated with $\left\{f_{n}\right\}_{1}^{\infty}$ and with radius of convergence $\rho$. Then $\rho<\infty$ and $F(\rho) \leq 1$, while 
(i) $F(\rho)<1$ iff $U(\rho)<\infty$, in which case $\lim _{n \rightarrow \infty} \rho^{n} u_{n}=0$;

(ii) If $F(\rho)=1$ and $\left\{f_{n}\right\}_{1}^{\infty}$ is aperiodic, then $\lim _{n \rightarrow \infty} \rho^{n} u_{n}=1 / \mu$, where $\mu$ is given by

$$
\mu:=\sum_{n=1}^{\infty} n f_{n} \rho^{n}=\rho F^{\prime}(\rho) \quad(\leq \infty) .
$$

REMARK 4.1.17. Theorem 4.1.16 and the second part of theorem 4.1.14 have been proved previously by De Bruijn \& Erdös (1951) in a slightly less general situation; they consider general solutions of the pure renewal equation, but suppose the $f_{n}$ "s to be all positive. For this case they show furthermore that the quantities $\rho$ and $\theta$ can be expressed in terms of the $f_{n}$ 's as follows:

$$
\text { (4.1.20) } \rho=\theta^{-1}=\sup \{x \geq 0 \mid F(x) \leq 1\} .
$$

However, this relation holds for all renewal sequences, as will be shown in a more general situation in the next section.

The class $R_{0}$ contains two easily recognizable subclasses, viz. the class of comp mon sequences $\left\{u_{n}\right\}_{0}^{\infty}$ (cf, definition 1.3.10) with $u_{0}=1$, and the class of bounded Kaluza sequences. Here a sequence $\left\{a_{n}\right\}_{0}^{\infty}$ is called a Kaluza sequence if $a_{0}=1, a_{n} \geq 0(n \in \mathbb{N})$ and $\left\{a_{n}\right\}_{0}^{\infty}$ is log-convex, i.e.

$$
a_{n}^{2} \leq a_{n-1} a_{n+1} \quad(n \in \mathbb{N}) .
$$

Note that in this case $a_{n}=0$ for all $n \in \mathbb{N}$ or $a_{n}>0$ for all $n \in \mathbb{N}$, and that $\left\{a_{n}\right\}$ is bounded iff it is nonincreasing. Kaluza (1928) proved the following theorem, the analogue of which for $C_{0}$ has been already given in theorem $1: 5.13$.

THEOREM 4.1.18. A comp mon sequence $\left\{u_{n}\right\}_{0}^{\infty}$ with $u_{0}=1$ is a bounded Kaluza sequence, and a bounded Kaluza sequence is in $R_{0}$.

The bounded Kaluza sequences can be characterized as a subclass of $R_{0}$ in two somewhat similar ways. The first one is given by Kendall (1967), the second one seems to be new. 
THEOREM 4.1.19. Let $\left\{u_{n}\right\}_{0}^{\infty}$ be a sequence of positive numbers. Then $\left\{u_{n}\right\}$ is a bounded Kaluza sequence iff for all $t>0$ (or, equivalently, for all $t=1 / \mathrm{m}$, $m \in \mathbb{N})\left\{u_{n}^{t}\right\} \in R_{0}$, in which case $\left\{u_{n}^{t}\right\}$ is also a bounded kaluza sequence for all $t>0$.

Translating this result for $\left\{p_{n}\right\} \in C_{0}$, we have to consider $\gamma^{n} p_{n}$ for some $\gamma \in(0,1)$ rather than $p_{n}$, since $\Sigma p_{n}^{t}$ is not necessarily finite for all $t>0$.

THEOREM 4.1.20. Let $\left\{u_{n}\right\}_{0}^{\infty}$ be a sequence of positive numbers. For $l \in \mathbb{N}_{0}$ define the sequence $\left\{u_{n}(l)\right\}_{0}^{\infty}$ by

$$
u_{n}(\ell):=u_{\ell+n} / u_{\ell} \quad\left(n \in \mathbb{N}_{0}\right) .
$$

Then $\left\{u_{n}\right\}$ is a bounded Kaluza sequence iff $\left\{u_{n}(l)\right\} \in R_{0}$ for all $\ell \in \mathbb{N} 0^{\text {, in }}$ which case $\left\{u_{n}(\ell)\right\}$ is also a bounded Kaluza sequence for all $\ell \in \mathbb{N}_{0}$ 。

PROOF. Let $\left\{u_{n}\right\}$ be a bounded Kaluza sequence and take $\ell \in \mathbb{N}_{0}$ fixed. As $\left\{u_{n}\right\}_{0}^{\infty}$ is nonincreasing, $u_{n}(l)$ is bounded by 1 . Furthermore, $\left\{u_{n}(l)\right\}$ is logconvex, as is seen from $(n \in \mathbb{N})$

$$
u_{n}(\ell)^{2}=u_{\ell}^{-2} u_{\ell+n}^{2} \leq u_{\ell}^{-2} u_{\ell+n-1} u_{\ell+n+1}=u_{n-1}(\ell) u_{n+1}(\ell) .
$$

Conversely, let $\left\{u_{n}(l)\right\} \in R_{0}$ for all $\ell \in \mathbb{N}_{0}$. Taking $l=0$, we see that $\left\{u_{n}\right\} \in R_{0}$, and hence $\left\{u_{n}\right\}$ is bounded. The sequence $\left\{f_{n}(l)\right\}_{1}^{\infty}$, with which $\left\{u_{n}(l)\right\}$ is associated, consists of nonnegative numbers. Now, calculating $f_{2}(l)$, we get

$$
f_{2}(\ell)=u_{2}(\ell)-u_{1}(\ell)^{2}=u_{\ell}^{-2}\left\{u_{\ell} u_{\ell+2}-u_{\ell+1}^{2}\right\},
$$

from which it follows that the nonnegativity of $f_{2}(l)$ for all $\ell \in \mathbb{I N}$ implies the log-convexity of $\left\{u_{n}\right\}_{\circ}^{\infty}$. Hence $\left\{u_{n}\right\}$ is a bounded Kaluza sequence.

REMARK 4.1.21. If we define the sequence $\left\{u_{n}(l)\right\}$ somewhat differently, viz.

$$
u_{0}(\ell)=1, u_{n}(\ell)=u_{\ell+n} \quad(n \in \mathbb{N}),
$$

then we get a weaker result: $\left\{u_{n}\right\}$ is a bounded Kaluza sequence iff $\left\{u_{n}(l)\right\}_{0}^{\infty}$ is a bounded Kaluza sequence for all $\ell \in \mathbb{N}_{0}$.

Finally, we consider the occurrence of a compound geometric lattice distribution in a stochastic process. By lemma 4.1 .1 (i), theorems 4.1 .5 and 4.1 .6 
(i) yield, rather artificially, an interpretation of $\left\{p_{n}\right\} \in C_{0}$ in a pure, transient renewal process and in a Markov chain, respectively. In the first case, however, we can say a little more. Using the notation of definition 4.1.4, we denote the (pure) renewal sequence corresponding to $\left\{s_{n}\right\}$ (cf. theorem 4.1 .5$)$ by $\left\{u_{n}\right\}_{0}^{\infty}$, and define

$$
\mathrm{N}:=\#\left(\left\{\mathrm{n} \in \mathbb{N} \mid \mathrm{S}_{\mathrm{n}}<\infty\right\}\right),
$$

i.e. $N$ is the total number of renewal epochs $\left(S_{0}=0\right.$ not counted). Then $N$ is finite with probability one:

$$
\mathrm{P}(\mathrm{N}=\infty)=\mathrm{P}\left(\forall_{\mathrm{n} \in \mathbb{N}} \mathrm{S}_{\mathrm{n}}<\infty\right)=\lim _{\mathrm{n} \rightarrow \infty} \mathrm{P}\left(\forall_{\mathrm{k} \leq \mathrm{n}} \mathrm{T}_{\mathrm{k}}<\infty\right)=\lim _{\mathrm{n} \rightarrow \infty} \mathrm{f}^{\mathrm{n}}=0 .
$$

In fact, $\mathrm{N}$ has a geometric distribution with parameter $\mathrm{f}$ :

$$
\mathrm{P}(\mathrm{N}=\mathrm{k})=\mathrm{P}\left(\mathrm{S}_{\mathrm{k}}<\infty ; \mathrm{T}_{\mathrm{k}+1}=\infty\right)=(1-f) \mathrm{f}^{\mathrm{k}} \quad\left(\mathrm{k} \in \mathbb{N}_{\mathrm{O}}\right)
$$

Now we can define the duration $D$ of the process $\left\{S_{n}\right\}$ as $D=S_{N}$. Its distribution is easily calculated:

$$
\begin{aligned}
P(D=n) & =\sum_{k=0}^{\infty} P\left(S_{N}=n ; N=k\right)=\sum_{k=0}^{\infty} P\left(S_{k}=n ; T_{k+1}=\infty\right)= \\
& =\sum_{k=0}^{\infty} P\left(S_{k}=n\right)(1-f)=(1-f) u_{n} \quad\left(n \in \mathbb{N}_{0}\right)
\end{aligned}
$$

from which by lemma 4.1.1 we obtain the following interpretation of $\left\{p_{n}\right\} \in C_{0}$.

THEOREM 4.1.22. Let $\left\{\mathrm{p}_{\mathrm{n}}\right\}_{0}^{\infty}$ be a lattice distribution. Then $\left\{\mathrm{p}_{\mathrm{n}}\right\} \in \mathrm{C}_{0}$ iff there exists a pure, transient renewal process $\left\{s_{n}\right\}_{0}^{\infty}$ such that $\left\{p_{n}\right\}$ is the distribution of the duration $D$ of the process.

\subsection{Generalized renewal sequences; classes $R_{\alpha}(0<\alpha<1)$}

In this section we study the distributions in $C_{\alpha}$ for $0<\alpha<1$, by considering slightly more general classes $R_{\alpha}$. As these are defined in such a way that there exists a relation between $C_{\alpha}$ and $R_{\alpha}$ (to be given in lemma 4.2.4), similar to that between $C_{0}$ and $R_{0}$, we can (and will) confine ourselves to studying the $R_{\alpha}$ 's. As in section 1, the properties of $R_{\alpha}$ can easily be translated into properties of $C_{\alpha}$. We define the following classes $R_{\alpha}$, employing the notation used for the $C_{\alpha}{ }^{\prime}$ s rather than that used for $R_{0}$. 
DEFINITION 4.2.1. For $0<\alpha<1$ a sequence $\left\{u_{n}\right\}_{0}^{\infty}$ of real numbers with $u_{0}=1$ is said to be in the class $R_{\alpha}$ if there exist nonnegative quantities $r_{n}(\alpha)$ ( $n \in \mathbb{N}_{0}$ ) satisfying

$$
\text { (4.2.1) } \quad r(\alpha):=\sum_{n=0}^{\infty} r_{n}(\alpha) \leq \frac{1}{1-\alpha} .
$$

such that

$$
\text { (4.2.2) } \frac{1-\alpha^{n+1}}{1-\alpha} u_{n+1}=\sum_{k=0}^{n} r_{k}(\alpha) u_{n-k} \quad\left(n \in \mathbb{N}_{0}\right) .
$$

We could consider more general classes by dropping condition (4.2.1). Many results to be proved for $R_{\alpha}$ will also hold in that case. But in view of probabilistic interpretations and because for a sequence $\left\{u_{n}\right\}$ that satisfies $(4.2 .2)$ and has a gf with positive radius of convergence, we can choose $\gamma \in(0,1)$ such that $\left\{\gamma^{n} u_{n}\right\}$ is bounded and still satisfies $(4.2 .2)$, we will only consider bounded sequences $\left\{u_{n}\right\}$. This results in the classes $R_{\alpha}$, as is seen from the following lemma.

LEMMA 4.2.2. Let $0<\alpha<1$ and let $\left\{u_{n}\right\}_{0}^{\infty}$ be a sequence with $u_{0}=1$ and satisfying (4.2.2) with nonnegative $r_{n}(\alpha)^{\prime} s\left(n \in \mathbb{N}_{0}\right)$. Then $\left\{u_{n}\right\}$ is boundediff (4.2.1) holds (i.e. $\left\{u_{n}\right\} \in R_{\alpha}$ ), in which case for all $k \in \mathbb{N}_{0}$
$(4.2 .3)$

$$
u_{k} \leq \prod_{l=1}^{k}\left(1-\alpha^{l}\right)^{-1}
$$$$
\left(\leq \prod_{\ell=1}^{\infty}\left(1-\alpha^{\ell}\right)^{-1}<\infty\right)
$$

PROOF. Let $\left\{u_{n}\right\}$ be bounded. Then its gf U exists on $[0,1)$. Taking gf's in $(4.2 .2)$ we get

$$
(4.2 .4) \quad \frac{U(z)-U(\alpha . z)}{(1-\alpha) z}=U(z) R_{\alpha}(z) \quad(0 \leq z<1) .
$$

and hence

$$
z R_{\alpha}(z)=\{1-U(\alpha z) / U(z)\} /(1-\alpha) \leq 1 /(1-\alpha) \quad(0 \leq z<1) .
$$

Letting $z+1$, by the monotone convergence theorem we see that $r(\alpha) \leq 1 /(1-\alpha)$. Conversely, let $r(\alpha) \leq 1 /(1-\alpha)$. Suppose $(4.2 .3)$ to be true for $k=1, \ldots, n$. Then

$u_{n+1}=\frac{1-\alpha}{1-\alpha^{n+1}} \sum_{k=0}^{n} r_{k}(\alpha) u_{n-k} \leq \frac{1-\alpha}{1-\alpha^{n+1}} \sum_{k=0}^{n} r_{k}(\alpha) \prod_{\ell=1}^{n}\left(1-\alpha^{\ell}\right)^{-1} \leq \prod_{\ell=1}^{n+1}\left(1-\alpha^{\ell}\right)^{-1}$, 
which is less than

$$
\prod_{\ell=1}^{\infty}\left(1-\alpha^{\ell}\right)^{-1}=\exp \left[\sum_{\ell=1}^{\infty} \sum_{j=1}^{\infty} \frac{1}{j} \alpha^{\ell j}\right] \leq \exp \left[\alpha(1-\alpha)^{-2}\right]<\infty .
$$

REMARK 4.2.3. The inequality (4.2.3) cannot easily be improved, as is seen from the following example (cf. the boundary distribution from definition 2.2.2). Define $\left\{\tilde{u}_{n}\right\}_{0}^{\infty}$ as the sequence in $R_{\alpha}$ corresponding to $r_{0}(\alpha)=1 /(1-\alpha)$, $r_{n}(\alpha)=0 \quad(n \in \mathbb{N})$.

Then from $(4.2 .2)$ it follows that

$$
(4.2 .5) \quad\left(1-\alpha^{n+1}\right) \tilde{u}_{n+1}=\tilde{u}_{n} \quad\left(n \in \mathbb{N}_{0}\right)
$$

and hence

$$
\text { (4.2.6) } \quad \tilde{u}_{n}=\prod_{k=1}^{n}\left(1-\alpha^{k}\right)^{-1} \quad\left(n \in \mathbb{N}_{0}\right)
$$

one easily verifies that $C_{\alpha}$ is related to $R_{\alpha}$ in the following way (cf. lem$\operatorname{ma} 4.1 .1)$.

LEMMA 4.2.4. Let $0<\alpha<1$.

(i) If $\left\{\mathrm{p}_{\mathrm{n}}\right\} \in \mathrm{C}_{\alpha}$ and $\mathrm{u}_{\mathrm{n}}:=\mathrm{p}_{\mathrm{n}} / \mathrm{p}_{0}\left(\mathrm{n} \in \mathbb{N}_{0}\right)$, then $\left\{\mathrm{u}_{\mathrm{n}}\right\} \in R_{\alpha}$.

(ii) If $\left\{u_{n}\right\} \in R_{\alpha}$ and $p_{n}:=\gamma^{n} u_{n} / U(\gamma)$, where $0<\gamma<1$, then $\left\{p_{n}\right\} \in C_{\alpha}$.

It may be noted that if $x(\alpha)<1 /(1-\alpha)$, then in (ii) we can take $\gamma=1$.

This follows from the following property of $R_{\alpha}$.

THEOREM 4.2.5. Let $0<\alpha<1$ and let $\left\{u_{n}\right\} \in R$. Then $u:=\sum_{n=0}^{\infty} u_{n}$ is finite iff $x(\alpha)<1 /(1-\alpha)$, in which case

$(4.2 .7) \quad u=U(\alpha) /\{1-(1-\alpha) x(\alpha)\}$

PROOF" Let $0<\alpha<1$ and let $\left\{u_{n}\right\} \in R_{\alpha}$. Then relation (4.2.4) holds for all $|z|<1$, and can be written in the form

$$
U(z)=\left\{1-(1-\alpha) z R_{\alpha}(z)\right\}^{-1} U(\alpha z) \quad(|z|<1)
$$

Now, letting $z \uparrow 1$ and applying the monotone convergence theorem concludes the proot. 
Studying the $R_{\alpha}$ "s further, we first observe that many properties of the $C_{\alpha}$ 's can be extended to the $R_{\alpha}$ "s. For instance, using lemas 4.2 .4 and 4.2 .2 , one easily shows that

$$
(4.2 .8) \quad R_{\alpha} \subset R_{\beta} \quad(0 \leq \alpha<\beta<1) .
$$

Specifically, it follows that every $R_{\alpha}$ contains $R_{0}$ as a subclass, $i$.e. the sequences in $R_{\alpha}$ can be considered as generalized renewal sequences. Now, in the remainder of this section, we mainly investigate to what extent the propexties of $R_{0}$, summaxized in the preceding section, can be generalized to the larger classes $R_{\alpha}$. To this end we first state a useful relation of $R_{\alpha}$ with the (pure) renewal sequences, and next a more direct relation between $R_{\alpha}$ and certain delayed renewal sequences.

THEOREM 4.2.6. Let $0<\alpha<1$, and let $\left\{u_{n}\right\}_{0}^{\infty}$ be a sequence with $u_{0}=1$ and gf $U$. Define the sequence $\left\{u_{n}(\alpha)\right\}_{0}^{\infty}$ by its $g f U_{\alpha}(z):=U(z) / U(\alpha z)$, or recursively by

$\left.(4.2 .9) \quad u_{n}=\sum_{k=0}^{n} \alpha u_{k} u_{n-k}(\alpha) \quad(n \in \mathbb{N})_{0}\right)$.

Then $\left\{u_{n}\right\} \in R_{\alpha}$ iff $\left\{u_{n}(\alpha)\right\} \in R_{0}$.

PROOF: Let $\left\{u_{n}\right\} \in R_{\alpha}$ and take $\gamma \in(0,1)$. Then by lemma 4.2 .4 the relation between $C_{\alpha}$ and $C_{o}$, given by theorem 2.4 .5 , implies that $\left\{\gamma^{n} u_{n}(\alpha)\right\} \in R_{0}$. It follows that $\left\{u_{n}(\alpha)\right\}$ satisfies the pure renewal equation. Furthemore, since $\left\{u_{n}\right\}$ is bounded (Lemma 4.2 .2$)$ and as by $(4.2 .9)$ we have $u_{n}(\alpha) \leq u_{n}\left(n \in \mathbb{N}_{0}\right)$, $\left\{u_{n}(\alpha)\right\}$ is bounded, and on account of lemma 4.1 .3 we conclude that $\left\{u_{n}(\alpha)\right\} \in R_{0}$

Conversely, let $\left\{u_{n}(\alpha)\right\} \in R_{0}$. Then, similarly, it can be shown that $\left\{\gamma^{n} u_{n}\right\} \in R_{\alpha}$ for all $\gamma \in(0,1)$, and hence $\left\{u_{n}\right\}$ satisfies $(4.2 .2)$ with nonnegative $x_{n}(\alpha)$ " $s$. Now, in view of lemma 4.2 .2 , for $\left\{u_{n}\right\}$ to be in $R_{\alpha} i t$ is sufficient that $\left\{u_{n}\right\}$ is bounded. To show this, we prove (4.2.3), or, equivalently, $u_{n} \leq \tilde{u}_{n}$ (cf. remark 4.2.3). By (4.2.5) and mathematical induction, $\left\{\widetilde{u}_{n}\right\}$ is seen to satisfy

(4.2.10) $\tilde{u}_{n}=\sum_{k=0}^{n} a^{k} \tilde{u}_{k} \quad\left(n \in \mathbb{N}_{0}\right)$.

Hence, assuming that $u_{k} \leq \widetilde{u}_{k}$ for $k=0,1, \ldots, n-1$, by $(4.2 .9)$ and the fact that $u_{n}(\alpha) \leq 1\left(n \in \mathbb{N}_{0}\right)$ we can estimate $u_{n}$ as follows: 


$$
\begin{aligned}
u_{n} & =\left(1-\alpha^{n}\right)^{-1} \sum_{k=0}^{n-1} \alpha^{k} u_{k} u_{n-k}(\alpha) \leq\left(1-\alpha^{n}\right)^{-1} \sum_{k=0}^{n-1} \alpha^{k} \tilde{u}_{k}= \\
& =\left(1-\alpha^{n}\right)^{-1} \tilde{u}_{n-1}=\tilde{u}_{n} .
\end{aligned}
$$

It follows that $\left\{u_{n}\right\}$ is bounded, and the theorem is proved.

THEOREM 4.2 .7 . Let $0<\alpha<1$.

(i) If $\left\{u_{n}\right\} \in R_{\alpha}$ and if $v_{n}:=u_{n} / U(\alpha) \quad\left(n \in \mathbb{N}_{0}\right)$, then $\left\{v_{n}\right\}_{0}^{\infty} \in R$, associated with $\left\{b_{n}\right\}_{0}^{\infty}$ and $\left\{f_{n}\right\}_{1}^{\infty}$ given by

$(4.2 .11) \quad b_{n}=\alpha v^{n} v_{n}\left(n \in \mathbb{N}_{0}\right), f_{n}=(1-\alpha) x_{n-1}(\alpha) \quad(n \in \mathbb{N})$.

(ii) If $\left\{v_{n}\right\} \in R$ is associated with $\left\{b_{n}\right\}$ and $\left\{f_{n}\right\}$ such that $b_{0}>0$ and $b_{n}=\alpha v_{n}\left(n \in \mathbb{N}_{0}\right)$, and if $u_{n}:=v_{n} / v_{0}\left(n \in \mathbb{N}_{0}\right)$, then $\left\{u_{n}\right\} \in R_{\alpha}$.

PROOF. Let $\left\{u_{n}\right\} \in R_{\alpha}$ and $v_{n}:=u_{n} / U(\alpha)$ (n $\left.\in \mathbb{N}_{0}\right)$. Then by definition 4.2 .1 $\left\{v_{n}\right\}_{0}^{\infty}$ is easily seen to satisfy the renewal equation (4.1.4) with $\left\{b_{n}\right\}$ and $\left\{f_{n}\right\}$ given by $(4.2 .11)$. It follows that $b=1$ and $f=(1-\alpha) r(\alpha) \leq 1$, and hence that $\left\{\mathrm{v}_{\mathrm{n}}\right\} \in R$.

Finally, (ii) can be shown in a similar way.

REMARK 4.2.8. For $\left\{v_{n}\right\} \in R$, associated with $\left\{b_{n}\right\}$ and $\left\{f_{n}\right\}$, the condition $b_{n}=\alpha^{n} v_{n}\left(n \in \mathbb{N}_{0}\right)$ can be reformulated as a relation between $\left\{b_{n}\right\}$ and $\left\{f_{n}\right\}$ :

$(4.2 .12) \quad\left(1-\alpha^{n}\right) b_{n}=\sum_{k=1}^{n} \alpha^{k} f_{k} b_{n-k} \quad(n \in \mathbb{N})$

(i.e. in case $v_{0}>0$ and $V(\alpha)=1:\left\{b_{n}\right\}_{0}^{\infty} \in C_{\alpha}$ with corresponding $r_{n}(\alpha)^{\prime}$ s given by $r_{n}(\alpha)=(1-\alpha)^{-1} \alpha^{n+1} E_{n+1}$ ). This can be seen from (4.1.5) (replace $z$ by $\alpha z$ ).

REMARK 4.2.9. Using theorem 4.2 .6 one easily proves the following implication:

$(4.2 .13) \quad U \in R_{0} \Rightarrow W(z):=U(z) U(\alpha z) \in R_{\alpha}$.

Now, in view of $(4.1 .6)$, the sequences $\left\{w_{n}\right\}$ in $R_{\alpha}$, thus obtained, are of the form $w_{n}=U(a) v_{n}$, where $\left\{v_{n}\right\} \in R$ is such that the sequences $\left\{b_{n}\right\}$ and $\left\{f_{n}\right\}$, that $\left\{v_{n}\right\}$ is associated with, satisfy $B(z)=\{1-F(\alpha)\} /\{1-F(\alpha z)\}$, i.e. 
$(4.2 .14) \quad b_{n}=(1-F(\alpha)) \alpha^{n} \sum_{k=0}^{n} f_{n}^{* k} \quad\left(n \in \mathbb{N}_{0}\right)$

Relation (4.2.14) seems somewhat more tractable than (4.2.12), but, unfortunately, not all sequences in $R_{\alpha}$ can be obtained in this way, i.e. the converse of (4.2.13) is not true. Indeed, every $W \in R_{\alpha}$ can be written as $U(z) U(\alpha z)$, but generally $U$ will belong to $R_{\alpha}$ and not necessarily to $R_{0}$ (cf. (3.1.14) and lemma 3.1 .8 ).

The relation between $R_{\alpha}$ and $R$ in theorem 4.2 .7 yields interpretations of $\left\{u_{n}\right\} \in R_{\alpha}$ in a renewal process and Markov chain (cf. theorems 4.1 .5 and $4.1 .6(i i)$ ). J. Wijngaard (personal communication) gave a third interpretation, where $u_{n}$ appears as the expected number of particles at time $n$ in a complicated "Markov branching-process". Although theorem 4.2 .7 will be applied when we consider the asymptotic behaviour of $\left\{u_{n}\right\} \in R_{\alpha}$, these interpretations do not yield easy proofs of inequalities or closure properties for $R_{\alpha}$ of the kind one has for $R_{0}$. For instance, if $\left\{u_{n}^{(i)}\right\} \in R_{\alpha}(i=1,2)$ and $v_{n}^{(i)}:=u_{n}^{(i)} / U_{i}(\alpha)\left(i=1,2 ; n \in \mathbb{N}_{0}\right)$, then $\left\{v_{n}^{(i)}\right\} \in R(i=1,2)$, and hence, by the Markov-chain interpretation, also $\left\{v_{n}\right\} \in R_{\text {, where }} v_{n}:=$ $:=v_{n}^{(1)} v_{n}^{(2)}\left(n \in \mathbb{N}_{0}\right)$. But since the $\left\{b_{n}\right\}$ and $\left\{f_{n}\right\}$, that $\left\{v_{n}\right\}$ is associated with, are not tractable, we cannot go back to $R_{\alpha}$.

Using the relation between $R_{\alpha}$ and $R_{0}$ from theorem 4.2 .6 , we can say a little more, but the results are only formal and rather obscure. We give one example, where we make use of theorem 4.1.7(i.i.).

THEOREM 4.2.10. If $\left\{u_{n}\right\}$ and $\left\{v_{n}\right\} \in R_{\alpha}$, and if $\left\{u_{n}(\alpha)\right\}$ and $\left\{v_{n}(\alpha)\right\}$ are defined by

$$
u_{n}=\sum_{k=0}^{n} \alpha u_{k} u_{n-k}(\alpha), v_{n}=\sum_{k=0}^{n} \alpha v_{k}^{k} v_{n-k}(\alpha) \quad\left(n \in \mathbb{N}_{0}\right)
$$

then the sequence $\left\{w_{n}\right\}^{\infty}$, defined by

$$
w_{0}=1, w_{n}=\sum_{k=0}^{n} \alpha w_{k} u_{n-k}(\alpha) v_{n-k}(\alpha) \quad(n \in \mathbb{N})
$$

is also in $R_{\alpha}$.

Not able to prove inequalities for $\left\{u_{n}\right\} \in R_{\alpha}$ using a probabilistic interpretation, we adapt the analytic proof of the first inequality in theorem 
4.1.7(i). To this end we need the following lemma from DeBruijn \& Erdös (1951).

LEMMA 4.2.11. If $\left\{v_{n}\right\}_{0}^{\infty}$ is a sequence of real numbers with $v_{0}=1$ and satisfying

$(4.2 .15) \quad v_{n+1}=\sum_{k=0}^{n} r_{k, n} v_{n-k} \quad\left(n \in \mathbb{N}_{0}\right)$

where $r_{k, n} \geq 0\left(k \in \mathbb{N}_{0} ; n=k, k+1, \ldots\right)$ and $r_{k, n}$ is nondecreasing in $n$ for all $k \in \mathbb{N}_{0}$, then for $\mathrm{n} \in \mathbb{N}_{\mathrm{o}}$

$(4.2 .16) \quad v_{n+k} \geq v_{n} v_{k} \quad\left(k \in \mathbb{N}_{0}\right)$.

PROOF. We use mathematical induction with respect to $n$. For $n=0(4.2 .16)$ is trivial. Now suppose that $(4.2 .16)$ holds for $n=1, \ldots, N$, then for all $k \in \mathbb{N}_{0}$

$$
\begin{aligned}
v_{N+1+k} & =\sum_{\ell=0}^{N+k} x_{\ell, N+k} v_{N+k-\ell} \geq \sum_{\ell=0}^{N} r_{\ell, N+k} v_{N-\ell} v_{k} \geq \\
& \geq v_{k} \sum_{\ell=0}^{N} x_{\ell, N} v_{N-\ell}=v_{N+1} v_{k},
\end{aligned}
$$

and $(4.2 .16)$ is proved for $\mathrm{n}=\mathrm{N}+1$.

Before stating the generalized inequality, we introduce the following notation:

(4.2.17) $h_{n}(\alpha):=\prod_{k=1}^{n} \frac{1-\alpha^{k}}{1-\alpha} \quad\left(n \in \mathbb{N}_{0}: 0 \leq \alpha<1\right)$.

THEOREM 4.2.12. If $0 \leq \alpha<1$ and $\left\{u_{n}\right\} \in R_{\alpha}$, then the following inequality holds:

$$
\text { (4.2.18) } \frac{h_{n+k}(\alpha)}{h_{n}(\alpha) h_{k}(\alpha)} u_{n+k} \geq u_{n} u_{k} \quad\left(n, k \in \mathbb{N}_{0}\right) .
$$

PROOF. Let $\left\{u_{n}\right\} \in R_{\alpha}$. Define $v_{n}:=h_{n}(\alpha) u_{n}\left(n \in \mathbb{N}_{0}\right)$, then $v_{0}=1$ and by $(4.2 .2)$ 


$$
v_{n+1}=h_{n}(\alpha) \frac{1-\alpha}{1-\alpha} u_{n+1}=\sum_{k=0}^{n} x_{k}(\alpha) \frac{h_{n}(\alpha)}{h_{n-k}(\alpha)} v_{n-k} \quad\left(n \in \mathbb{N}_{0}\right)
$$

i.e. $\left\{v_{n}\right\}_{0}^{\infty}$ satisfies $(4.2 .15)$ with $r_{k, n}$ given by

$$
r_{k, n}=r_{k}(\alpha) h_{n}(\alpha) / h_{n-k}(\alpha)=r_{k}(\alpha) \prod_{j=0}^{k-1} \frac{1-\alpha \alpha^{n-j}}{1-\alpha}\left(k \in \mathbb{N}_{0} ; n=k, k+1, \ldots\right) 。
$$

Obviously, $r_{\mathrm{k}, \mathrm{n}}$ is nonnegative and nondecreasing in $\mathrm{n}$, from which by lemma 4.2 .11 it follows that $(4.2 .16)$, and hence $(4.2 .18)$, holds.

COROLLARY 4.2.13. If $0 \leq \alpha<1$ and $\left\{u_{n}\right\} \in R_{\alpha}$, then $u_{n}$ can be bounded as folLows:

$(4.2 .19) \quad \frac{u_{1}^{n}}{h_{n}(\alpha)} \leq u_{n} \leq \frac{(1-\alpha)^{-n}}{h_{n}(\alpha)} \quad\left(n \in \mathbb{N}_{0}\right)$.

PROOF: The first part of the inequality follows by iteration of (4.2.18), while the second part is a reformulation of $(4.2 .3)$.

we note that both inequalities in (4.2.19) become equalities if $u_{n}=\widetilde{u}_{n}$ (cf. remark 4.2.3). Further, theorem 4.2 .12 implies the following result about the zeros of $\left\{u_{n}\right\} \in R_{\alpha}$. It can also be obtained from theorem 1.5.7 and the fact that $R_{\alpha} \subset R_{1}$, where $R_{1}$ is an extension of $C_{1}$ to be defined in the next section.

COROLLARY 4.2.14. If $0 \leq \alpha<1$ and $\left\{u_{n}\right\} \in R_{\alpha}$, then the following implication holds:

$$
\left[u_{n}>0 \text { and } u_{k}>0\right] \Rightarrow u_{n+k}>0 \quad\left(n, k \in \mathbb{N}_{0}\right)
$$

Consequently, if $u_{1}>0$ then $u_{n}>0$ for all $n \in \mathbb{N}_{0}$.

The upper bound for $u_{n}$ given in $(4.2,19)$, is independent of $\left\{u_{n}\right\} \in R \alpha^{\text {. Now, }}$ using the relation between $R_{\alpha}$ and $R$, given in theorem 4.2 .7 , and observing that $v_{n} \leq 1\left(n \in \mathbb{N}_{0}\right)$ if $\left\{v_{n}\right\} \in R$, we see that if $\left\{u_{n}\right\} \in R_{\alpha}$ then

$$
(4.2 .20) \quad u_{n} \leq u(\alpha) \quad\left(n \in \mathbb{N}_{0}\right)
$$

i.e. we have an upper bound for $u_{n}$, independent of $n \in \mathbb{N}_{0}$. 
Furthermore, theorem 4.2 .7 can be used to generalize the renewal theorem for $R_{0}$ (theorem 4.1.2(i) and (ii) with $b_{n}=\delta_{0, n}$ ') to the $R_{\alpha}{ }^{\prime} s$. The first part has already been generalized in theorem 4.2 .5 , the second part will be considered after the following lemma concerning the period of $\left\{u_{n}\right\} \in R_{\alpha}$.

LEMMA 4.2.15. Let $0<\alpha<1$, Let $\left\{u_{n}\right\} \in R_{\alpha}$, and suppose that the $r_{n}(\alpha)^{\prime} s_{\text {, }}$ corresponding to $\left\{u_{n}\right\}$, are not all zero. Then the period $d$ of $\left\{u_{n}\right\}$ is equal to that of the sequence $\left\{r_{n-1}(\alpha)\right\}_{1}^{\infty}$, and

$(4.2 .21) \not\left(\left\{n \in \mathbb{N} \mid u_{n d}=0\right\}\right)<\infty$.

PROOF. Let $\left\{u_{n}\right\} \in R_{\alpha}$ with period $d$, and let $\delta$ be the period of $\left\{x_{n-1}(\alpha)\right\}_{1}^{\infty}$. since by $(4.2 .2)$ the following inequality holds:

$$
\frac{1-\alpha^{n+1}}{1-\alpha} u_{n+1} \geq r_{n}(\alpha) \quad\left(n \in \mathbb{N}_{0}\right)
$$

we have

$$
\left\{\mathrm{n} \in \mathbb{N} \mid x_{n-1}(\alpha)>0\right\} \subset\left\{n \in \mathbb{N} \mid u_{n}>0\right\}
$$

and hence $\delta \geq$ d. As in the proof of theorem 2.3.4. by iteration of (4.2.4) we obtain the following expression for $U$ in terms of $R_{Q}$ :

$(4.2 .22) \quad \mathrm{U}(z)=\prod_{k=0}^{\infty}\left\{1-(1-\alpha) \alpha^{k} z R_{\alpha}\left(\alpha^{k} z\right)\right\}^{-1}$.

Now, as $r_{n-1}(\alpha)=0$ unless $\delta$ divides $n(n \in \mathbb{N})$, we see that each factor in the right-hand side of $(4.2 .22)$, and hence $u$, is a power series in $z$, i.e.

$$
\left\{n \in \mathbb{N} \mid u_{n}>0\right\} \subset\{k \delta \mid k \in \mathbb{N}\},
$$

so that $d \geq \delta$. It follows that $d=0$. To prove $(4.2 .21)$ we apply theorem 4.2.6, according to which the sequence $\left\{u_{n}(\alpha)\right\}_{0}^{\infty}$, with gf $U_{\alpha}(z):=U(z) / U(\alpha z)$, is in $R_{0}$. The $g f F$ of $\left\{f_{n}\right\}_{1}^{\infty}$, that $\left\{u_{n}(\alpha)\right\}$ is associated with, satisfies

$$
F(z)=1-1 / U_{\alpha}(z)=1-U(\alpha z) / U(z)=(1-\alpha) z R_{\alpha}(z),
$$

i.e. $f_{n}=(1-\alpha) x_{n-1}(\alpha) \quad(n \in \mathbb{N})$. Now by theorem 4.1.14 it follows that the period of $\left\{u_{n}(\alpha)\right\}$ is equal to $d$, and since by (4.2.9) we have

$$
u_{n} \geq u_{n}(\alpha) \quad\left(n \in \mathbb{N}_{0}\right)
$$

we obtain (4.2.21) from the corresponding result for $\left\{u_{n}(\alpha)\right\}($ cf. (4.1.17)). $\square$ 
THEOREM 4.2.16. Let $0<\alpha<1$ and let $\left\{u_{n}\right\} \in R_{\alpha}$. Suppose that the $r_{n}(\alpha)^{\prime} \mathrm{s}$, corresponding to $\left\{u_{n}\right\}$, are such that $r(\alpha)=1 /(1-\alpha)$, and let $\left\{u_{n}\right\}$ have period $d$. Then $u_{\infty}:=\lim _{n \rightarrow \infty} u_{n d}$ exists, and $u_{\infty}$ is given by

$(4.2 .23) \quad u_{\infty}=(1-\alpha)^{-1} v(\alpha) \frac{d}{\mu}$,

where

$(4.2 .24) \quad \mu:=\sum_{n=0}^{\infty}(n+1) r_{n}(\alpha) \quad(\leq \infty)$.

PROOF. Let $\left\{u_{n}\right\} \in R_{\alpha}$. By the preceding lemma we have

$$
u_{n}=x_{n-1}(\alpha)=0 \text { if } n \notin\left\{l d \mid l \in \mathbb{N}_{0}\right\}
$$

Now, for the sequence $\left\{w_{n}\right\}_{0}^{\infty}$, with $w_{n}:=u_{n d}\left(n \in \mathbb{N}_{0}\right)$, it follows that for $n \in \mathbb{N}_{0}$

$$
\begin{aligned}
& \frac{1-\left(\alpha^{\alpha}\right)^{n+1}}{1-\alpha} w_{n+1}=\frac{1-\alpha}{1-\alpha} \frac{1-\alpha^{(n+1) d}}{1-\alpha} u_{(n+1) d}= \\
& =\frac{1-\alpha}{1-\alpha} \sum_{\ell=1}^{n+1} r_{\ell d-1}(\alpha) u_{(n+1-\ell) d}=\sum_{k=0}^{n} \frac{1-\alpha}{1-\alpha} x_{(k+1) d-1}(\alpha) w_{n-k} .
\end{aligned}
$$

Hence $\left\{w_{n}\right\} \in R_{\alpha} d$ and, if the $r_{n}(\alpha)^{d}$ 's, corresponding to $\left\{w_{n}\right\}$, are denoted by $\bar{r}_{n}\left(\alpha^{\alpha}\right) \quad\left(n \in \mathbb{N}_{0}\right)$, then

$$
\bar{x}_{n}(\alpha)=\frac{1-\alpha}{1-\alpha} x_{(n+1) \alpha-1}(\alpha) \quad\left(n \in \mathbb{N}_{0}\right) .
$$

Now, according to theorem $4.2 .7(i)$, the sequence $\left\{v_{n}\right\}_{0}^{\infty}$, with $v_{n}:=w_{n} / w(\alpha)$ $\left(n \in \mathbb{I N}_{0}\right)$, is a delayed renewal sequence, associated with

$$
b_{n}=\alpha v_{n}^{n d}\left(n \in \mathbb{N}_{0}\right) \text { and } f_{n}=\left(1-\alpha^{d}\right) \bar{r}_{n-1}(\alpha) \quad(n \in \mathbb{N})
$$

Since $\left\{\bar{x}_{n-1}\left(\alpha^{d}\right)\right\}_{1}^{\infty}$, and hence $\left\{f_{n}\right\}_{1}^{\infty}$, is aperiodic, and as

$$
f=\sum_{n=1}^{\infty} f_{n}=(1-\alpha) \sum_{n=1}^{\infty} r_{n d-1}(\alpha)=(1-\alpha) r(\alpha)=1,
$$

by the renewal theorem (theorem 4.1 .2 ) it follows that $v_{\infty}:=\lim _{n \rightarrow \infty} v_{n}$ exists, and that $\mathrm{v}_{\infty}=\mathrm{b} / \mu_{1}$, where $\mu_{1}:=\sum \mathrm{nf} \mathrm{n}_{\mathrm{n}}$ observing that in our case $\mathrm{b}=1$, 
$w(\alpha)=U(\alpha)$, and

$$
\mu_{1}=\sum_{n=1}^{\infty} n f_{n}=(1-\alpha) \sum_{n=1}^{\infty} n r_{n d-1}(\alpha)=(1-\alpha) d^{-1} \mu,
$$

with $\mu$ given by $(4.2 .24)$, we see that $u_{\infty}:=\lim _{n \rightarrow \infty} u_{n d}$ exists, and is equal to the expression in $(4.2 .23)$.

If the $r_{n}(\alpha)^{\prime} s$, corresponding to $\left\{u_{n}\right\} \in R$, are such that either $r(\alpha)<$ $1 /(1-\alpha)$ or $r(\alpha)=1 /(1-\alpha)$ and $\mu=\Sigma(n+1) r_{n}(\alpha)=\infty$, then $u_{n} \rightarrow 0$ as $n \rightarrow \infty$. In order to know how fast $u_{n}$ may tend to zero, we generalize the results of Kingman (1972) and DeBruijn \& Erdös (1951) for $R_{0}$ (cf. theorem 4.1.14 and remark 4.1.17) to $R_{\alpha}$. It turns out that, as in the case $\alpha=0,\left\{u_{n}\right\} \in R_{\alpha}$ tends to zero not faster than exponentially.

THEOREM 4.2.17. Let $0<\alpha<1$ and let $\left\{u_{n}\right\} \in R_{\alpha}$. Suppose that the $r_{n}(\alpha)^{\text {' }} \mathrm{s}$, corxesponding to $\left\{u_{n}\right\}$, are not all zero, and that $\left\{u_{n}\right\}$ has period d. Final$I y$, let $p$ denote the radius of convergence of the gf of $\left\{u_{n}\right\}_{0}^{\infty}$. Then

$$
\theta:=\lim _{n \rightarrow \infty} u_{n d}^{1 /(n d)}
$$

exists in $(0,1], \rho$ is finite, and

$$
\text { (4.2.25) } \quad \theta^{-1}=\rho=\sup \left\{x \geq 0 \mid(1-\alpha) x \mathbb{R}_{\alpha}(x) \leq 1\right\}=: \gamma_{\alpha} .
$$

Furthermore (cf. $(4.2 .17)$ )

$$
(4.2 .26) \quad u_{n} \leq \theta^{n} \frac{(1-\alpha)^{-n}}{h_{n}(\alpha)} \quad\left(n \in \mathbb{N}_{0}\right)
$$

so that also $\left\{\rho^{n} u_{n}\right\}_{0}^{\infty} \in R_{\alpha}$.

PROOF. Let $\left\{u_{n}\right\} \in R_{\alpha}$. We introduce the sequence $\left\{v_{n}\right\}$ by

$$
v_{n}:=-\log \left\{h_{n d}(\alpha) u_{n d}\right\},
$$

which is well defined for $n$ sufficiently large, as then $u_{\text {nd }}>0$ (cf.

$(4.2 .21))$. By inequality $(4.2 .18)$ it follows that $\left\{v_{n}\right\}$ is subadditive, i.e.

$$
v_{n+k} \leq v_{n}+v_{k}
$$

and hence (cf. Pólya \& Szegö (1970), I. Abschn, Kap. 3) $\lim _{n \rightarrow \infty} v_{n} / n$ exists in 
$[-\infty, \infty)$, and is equal to $\ell:=$ inf $v_{n} / n$. It follows that

$$
\lim _{n \rightarrow \infty}\left\{h_{n d}(\alpha) u_{n d}\right\}^{1 /(n d)}=\exp \left[-\lim _{n \rightarrow \infty} v_{n} /(n d)\right]=\exp [-\ell / d]
$$

from which, observing that for $0<\alpha<1$

$$
\log h_{n}(\alpha) \sim-n \log (1-\alpha) \quad(n \rightarrow \infty),
$$

we see that

$$
\theta:=\lim _{n \rightarrow \infty} u_{n d}^{1 /(n d)}=(1-\alpha) \exp [-\ell / \alpha]
$$

As $l<\infty$, we have $\theta>0$. On the other hand, in view of (4.2.19) we can write

$$
\ell=\inf \frac{-1}{n} \log \left\{h_{n d}(\alpha) u_{n d}\right\} \geq \inf \frac{-1}{n} \log (1-\alpha)^{-n d}=\alpha \log (1-\alpha),
$$

and hence $\theta \leq 1$. Further, as $v_{n} / n \geq \ell$ for all $n$, we have

$$
\left\{h_{n d}(\alpha) u_{n d}\right\}^{1 /(n d)} \leq \exp [-\ell / d]=\theta(1-\alpha)^{-1}
$$

which yields $(4.2 .26)$. In view of lemma 4.2 .2 we may now conclude that also $\left\{\theta^{-n} u_{n}\right\}_{0}^{\infty} \in R_{\alpha}$.

Finally, we turn to relation $(4.2 .25)$. As in the proof of corollary 4.1.15, it can be shown that $\rho=\theta^{-1}$. As $\theta>0, \rho$ is finite. From $(4.2 .2)$ it is seen that

$$
(4.2 .27) \quad u_{n+1} \geq(1-\alpha) r_{n}(\alpha) \quad\left(n \in \mathbb{N}_{0}\right) \text {. }
$$

and hence the radius of convergence of $R_{\alpha}$ is at least $\rho$. Now by $(4.2 .4)$ it follows that

$$
(1-\alpha) R_{\alpha}(x)=1-U(\alpha x) / U(x) \leq 1 \quad(0 \leq x<\rho) .
$$

and hence $\gamma_{\alpha} \geq \rho$. On the other hand, using mathematical induction, we prove that for $k \in \mathbb{N}_{0}$

$(4.2 .28) \quad u_{k} \leq \gamma_{\alpha}^{-k} \prod_{\ell=1}^{k}\left(1-\alpha^{\ell}\right)^{-1}:$

suppose that $(4.2 .28)$ holds for $k=1, \ldots, n$, then we can write

$$
\gamma_{\alpha}^{n+1} u_{n+1}=\gamma_{\alpha}^{n+1} \frac{1-\alpha}{1-\alpha^{n+1}} \sum_{k=0}^{n} r_{k}(\alpha) u_{n-k} \leq
$$




$$
\begin{aligned}
& \leq \gamma_{\alpha}^{n+1} \frac{1-\alpha}{1-\alpha^{n+1}} \sum_{k=0}^{n} r_{k}(\alpha) \gamma_{\alpha}^{-(n-k)} \prod_{\ell=1}^{n-k}\left(1-\alpha^{\ell}\right)^{-1} \leq \\
& \leq(1-\alpha) \gamma_{\alpha} R_{\alpha}\left(\gamma_{\alpha}\right)^{n+1} \prod_{\ell=1}^{n+1}\left(1-\alpha^{\ell}\right)^{-1} \leq \prod_{\ell=1}^{n+1}\left(1-\alpha^{\ell}\right)^{-1} .
\end{aligned}
$$

It follows that there exists $M>0$ such that $u_{n} \leq M \gamma_{\alpha}^{-n}$ for all $n \in \mathbb{N}_{0}$ and hence $\rho \geq \gamma_{\alpha}$. Since we already showed that $\gamma_{\alpha} \geq \rho$, we get (4.2.25).

REMARK 4.2.18. If $\left\{u_{n}\right\} \in R_{\alpha}$ with $u_{1}>0$, then $d=1$, and (4.2.19) yields a sharper lower bound for $\theta=\lim _{n \rightarrow \infty} u_{n}^{1 / n}$ than the value zero given in theorem 4.2.17:

(4.2.29) $\quad \theta \geq(1-\alpha) u_{1}$

The first part of theorem 4.2 .17 can be reformulated as follows:

(4.2.30) $\lim _{n \rightarrow \infty}\left\{\rho^{n d} u_{n d}\right\}^{1 /(n d)}=1$

Now, from the fact that $\left\{\rho \mathrm{n}_{n}\right\} \in R_{\alpha}$ (cf. the last part of theorem 4.2.17). we can obtain some more information about the asymptotic behaviour of $\left\{\rho^{\text {nd }} u_{n d}\right\}^{\infty}$. In fact, applying theorems 4.2 .5 and 4.2 .16 to $\left\{\rho^{n} u_{n}\right\}$, we get the following result, which, of course, is only interesting if $\rho>1$.

THEOREM 4.2.19. Consider the situation from the preceding theorem, and let $\sigma_{\alpha}$ denote the radius of convergence of $R_{\alpha}$. Then $\gamma_{\alpha}$ satisfies

$$
(1-\alpha) \gamma_{\alpha} R_{\alpha}\left(\gamma_{\alpha}\right) \leq 1
$$

while

(i) $(1-\alpha) \gamma_{\alpha} R_{\alpha}\left(\gamma_{\alpha}\right)<1$ iff $U(\rho)<\infty$, in which case $\lim _{n \rightarrow \infty} \rho^{n} u_{n}=0$;

(ii) If $(1-\alpha) \gamma_{\alpha} R_{\alpha}\left(\gamma_{\alpha}\right)=1$, or, equivalently, if either $\sigma_{\alpha}=\infty$ or $\sigma_{\alpha}<\infty$ and $(1-\alpha) \sigma_{\alpha} R_{\alpha}\left(\sigma_{\alpha}\right) \geq 1$, then

(4.2.31) $\lim _{n \rightarrow \infty} \rho^{n d} u_{n d}=(1-\alpha)^{-1} U(\alpha \rho) \frac{d}{\mu}$

where

$(4.2 .32) \quad \mu:=\sum_{n=0}^{\infty}(n+1) x_{n}(\alpha) \rho^{n+1} \quad(\leq \infty)$. 
To conclude the discussion of the $R_{\alpha}{ }^{\prime} s$, we give a sufficient condition in terms of the $r_{n}(\alpha)$ 's for $\left\{u_{n}\right\} \in R_{\alpha}$ to be unimodal. This is suggested by theorem 3.3.8, which gives, in fact, the same result for the extension $R_{1}$ of $C_{1}$, to be defined in the next section.

THEOREM 4.2.20. Let $0<\alpha<1$ and let $\left\{u_{n}\right\} \in R_{\alpha}$. Suppose that the sequence $\left\{x_{n}(\alpha)\right\}$, corresponding to $\left\{u_{n}\right\}$, is such that $\left\{r_{n}(\alpha) / \alpha\right\}_{0}^{n}$ is nonincreasing. Then $\left\{u_{n}\right\}_{0}^{\infty}$ is unimodal, i.e. $\left\{u_{n}-u_{n-1}\right\}_{0}^{\infty}$, with $u_{-1}:=0$, changes sign at most once. Furthermore, $\left\{u_{n}\right\}$ is nonincreasing iff $x_{0}(\alpha) \leq 1$.

PROOF. Let $\left\{u_{n}\right\} \in R_{\alpha}$ and define the sequence $\left\{a_{n}\right\}_{0}^{\infty}$ by

$$
a_{n}:=u_{n}-u_{n-1} \quad\left(n \in \mathbb{N}_{0}\right)
$$

Replacing in $(4.2 .2) \mathrm{n}$ by $\mathrm{n}-1$, we can write

$$
\frac{1-\alpha^{n+1}}{1-\alpha} u_{n}=\alpha \frac{1-\alpha^{n}}{1-\alpha} u_{n}+u_{n}=\alpha \sum_{k=0}^{n-1} r_{k}(\alpha) u_{n-1-k}+u_{n} \text {. }
$$

From this and $(4.2 .2)$ we obtain by subtraction

$$
\text { (4.2.33) } \frac{1-\alpha^{n+1}}{1-\alpha} d_{n+1}=\left(x_{0}(\alpha)-1\right) u_{n}+\sum_{k=0}^{n-1}\left\{r_{k+1}(\alpha)-\alpha x_{k}(\alpha)\right\}_{n-1-k}\left(n \in \mathbb{N}_{0}\right) .
$$

Now, if the sequence $\left\{x_{n}(\alpha) / \alpha^{n}\right\}$ is nonincreasing then

$$
x_{n+1}(\alpha) \leq \operatorname{ar}_{n}(\alpha) \quad\left(n \in \mathbb{N}_{0}\right)
$$

and hence by $(4.2 .33)$ it follows that $a_{n} \leq 0(n \in \mathbb{N}), i . e,\left\{u_{n}\right\}_{0}^{\infty}$ is nonincreasing, iff $x_{0}(\alpha) \leq 1$. Finally, if $x_{0}(\alpha)>1$, then, relation $(4.2 .33)$ being very similax to (3.3.15), we obtain the unimodality of $\left\{u_{n}\right\}$ along the lines of the proof of theorem 3.3.8.

REMARK 4.2.21. Neither the condition that $r(\alpha) \leq 1 /(1-\alpha)$ nor the nonnegativity of the $r_{n}(\alpha)^{\prime}$ 's is essential in the preceding theorem. If, however, the $x_{n}(\alpha)$ 's are nonnegative, then $\left\{r_{n}(\alpha) / \alpha^{n}\right\}$ nonincreasing implies that the radius of convergence $\sigma_{\alpha}$ of $R_{\alpha}$ satisfies $\sigma_{\alpha} \geq 1 / \alpha$. Hence, unimodality of sequences $\left\{u_{n}\right\} \in R_{\alpha}$, for which $\sigma_{\alpha}<1 / \alpha$, cannot be proved by theorem 4.2.20.

REMARK 4.2.22. Though $R_{0}$ contains many unimodal sequences, e.g. the bounded Kaluza sequences (cf. theorem 4.1.18), there exists no obvious analogue of 
theorem 4.2.20 for $R_{0}$. However, as for $\left\{u_{n}\right\} \in R_{0}$ we have $u_{n} \leq 1=u_{0}$, a unimodal renewal sequence is necessarily nonincreasing. Hence (cf. (4.1.5)) a necessary and sufficient condition in terms of $\left\{f_{n}\right\}_{1}^{\infty}$ for $\left\{u_{n}\right\} \in R_{0}$ to be unimodal is that $\left\{\sum_{k=1}^{n} f_{n}^{* k}\right\}_{1}^{\infty}$ is nonincreasing, but this condition is not very useful.

Finally, we mention an analogue of theorem 4.1 .22 for $C_{\alpha}$. Consider a delayed, transient renewal process $\left\{\mathrm{s}_{n}\right\}_{0}^{\infty}$, associated wi.th $\left\{\mathrm{b}_{\mathrm{n}}\right\}_{0}^{\infty}$ and $\left\{\mathrm{f}_{\mathrm{n}}\right\}_{1}^{\infty}$ such that $b=1$ (and so $f<1$ ). Let $\left\{v_{n}\right\}_{0}^{\infty}$ denote the corresponding delayed renewal sequence (cf. theorem 4.1.5). Then, as in the case of a pure, transient renewal process (cf. the end of section 1), it can be shown that the $r v$

$$
\mathrm{N}:=\not \#\left(\left\{\mathrm{n} \in \mathbb{N} \mid \mathrm{s}_{\mathrm{n}}<\infty\right\}\right)
$$

has a geometric distribution with parameter $f$, and that the distribution $\left\{p_{n}\right\}_{0}^{\infty}$ of the duration $D=S_{N}$ is given by

$$
p_{n}=(1-f) v_{n} \quad\left(n \in \mathbb{N}_{0}\right)
$$

Now in view of the relation between $R$ and $R_{\alpha}$ (cf. theorem 4.2 .7 and remark 4.2 .8 ) and the relation between $R_{\alpha}$ and $C_{\alpha}$ (cf. lemma 4.2.4), we easily obtain the following characterization of $C_{\alpha}$ for $0<\alpha<1$.

THEOREM 4.2.23. For $0<\alpha<1$ a lattice distribution $\left\{p_{n}\right\}_{0}^{\infty}$ is in $\mathcal{C}_{\alpha}$ iff there exists a delayed, transient renewal process $\left\{\mathrm{s}_{n}\right\}$, associated with $\left\{b_{n}\right\}$ and $\left\{f_{n}\right\}$ satisfying $b=1, b_{0}>0$ and

$$
\left(1-\alpha^{n}\right) b_{n}=\sum_{k=1}^{n} \alpha^{k} f_{k} b_{n-k} \quad(n \in \mathbb{N})
$$

such that $\left\{p_{n}\right\}$ is the probability distribution of the duration of $\left\{s_{n}\right\}$.

\subsection{An extension of $C_{1}$; the class $R_{1}$}

In this section we briefly consider an extension $R_{1}$ of $C_{1}$. The class $C_{1}$ is in many respects different from the classes $C_{\alpha}$ for $0 \leq \alpha<1$ (cf. chapter $2)$. As a consequence, it is not clear how to define $R_{1}$ analogous to $R_{\alpha}$ for $\alpha<1, i . e$. how to characterize the boundedness of the $R_{1}$-sequences in terms of the $r_{n}(1)^{\prime} \mathrm{s}$. Therefore we define $R_{1}$ as follows. 
DEFINITION 4.3.1. A sequence $\left\{u_{n}\right\}_{0}^{\infty}$ with $u_{0}=1$ is said to be in the class $R_{1}$ if $i t$ is bounded and if there exist nonnegative quantities $r_{n}(1) \quad\left(n \in \mathbb{N}_{0}\right)$ such that

(4.3.1) $\quad(n+1) u_{n+1}=\sum_{k=0}^{n} r_{k}(1) u_{n-k} \quad\left(n \in \mathbb{N}_{0}\right)$.

M.L.J. Hautus suggested the following generalization of our result on the boundedness of sequences $\left\{u_{n}\right\}$ satisfying (4.3.1) with $r_{n}(1) \geq 0\left(n \in \mathbb{N}_{0}\right)$; in fact, we proved the case $\varepsilon_{n}=0\left(n \in \mathbb{N}_{0}\right)$.

LEMMA 4.3.2. Let $\left\{u_{n}\right\}_{0}^{\infty}$ be a sequence with $u_{0}=1$ and satisfying (4.3.1) with nonnegative $x_{n}(1)$ " $s\left(n \in \mathbb{N}_{0}\right)$. Then $\left\{u_{n}\right\}$ is bounded if there exist $N \in \mathbb{N}$ and a sequence $\left\{\varepsilon_{n}\right\}_{0}^{\infty}$ satisfying

(4.3.2) $\varepsilon_{n} \geq 0 \quad\left(n \in \mathbb{N}_{0}\right), \sum_{n=0}^{\infty} \varepsilon_{n}<\infty$,

such that

(4.3.3) $\frac{1}{n+1} \sum_{k=0}^{n} x_{k}(1) \leq 1+\varepsilon_{n} \quad(n \geq N)$.

PROOF, Define $v_{n}:=\max \left\{u_{0}, u_{1}, \ldots, u_{n}\right\}$. Because of $(4.3 .3)$ we have for all $\mathrm{n} \geq \mathbb{N}$

$$
u_{n+1}=\frac{1}{n+1} \sum_{k=0}^{n} r_{k}(1) u_{n-k} \leq\left(1+\varepsilon_{n}\right) v_{n}
$$

since $v_{n+1}=\max \left\{v_{n}, u_{n+1}\right\},\left\{v_{n}\right\}_{0}^{\infty}$ is now seen to satisfy

$$
v_{n+1} \leq\left(1+\varepsilon_{n}\right) v_{n} \quad(n \geq N),
$$

or also

$$
v_{n+1} \leq v_{N} \prod_{k=N}^{n}\left(1+\varepsilon_{k}\right) \quad(n \geq N)
$$

As $\sum \varepsilon_{n}<\infty$, it follows that $\left\{v_{n}\right\}$, and hence $\left\{u_{n}\right\}$, is bounded.

REMARK 4.3.3. If $r_{n}(1) \leq 1$ for all $n \in \mathbb{N}_{O^{\prime}}$ or if there exist $\mathrm{k} \in \mathbb{N}$ and $\gamma<1$ such that $r_{n}(1) \leq \gamma$ for $n \geq k$, then (4.3.3) holds and so $\left\{u_{n}\right\}$ is bounded. However, if, for instance, $x_{n}(1)=1+\varepsilon$ for all $n \in \mathbb{N}_{0}(\varepsilon>0)$, then it follows that (use $(4.3 .5)$ ) 


$$
u(z)=(1-z)^{-1-\varepsilon}
$$

and hence

$$
u_{n}=\left(\begin{array}{c}
-1-\varepsilon \\
n
\end{array}\right)(-1)^{n}=\left(\begin{array}{c}
n+\varepsilon \\
n
\end{array}\right)=\prod_{k=1}^{n}(1+\varepsilon / k) \quad\left(n \in \mathbb{N}_{0}\right)
$$

which tends to $\infty$ as $n \rightarrow \infty$. Thus it turns out that condition (4.3.3) is not too bad.

The gf's $U$ and $R_{1}$ of $\left\{u_{n}\right\} \in R_{1}$ and $\left\{r_{n}(1)\right\}$, corresponding to $\left\{u_{n}\right\}$, exist for $|z|<1$, because $\left\{u_{n}\right\}$ is bounded and $\left\{r_{n}(1)\right\}$ satisfies

$$
(4.3 .4) \quad x_{n}(1) \leq(n+1) u_{n+1} \quad\left(n \in \mathbb{N}_{0}\right) .
$$

From $(4.3 .1)$ it follows that

$$
\text { (4.3.5) } \quad U^{*}(z)=U(z) R_{1}(z) \quad(|z|<1),
$$

from which by integration and analytic continuation of the result we obtain the following representation for $U \in R_{1}$ :

$$
\text { (4.3.6) } \quad u(z)=\exp \left[\sum_{n=0}^{\infty} \frac{r_{n}(1)}{n+1} z^{n+1}\right] \quad(|z|<1) .
$$

Letting $z \uparrow 1$ we get the following analogue of theorems $4.1 .2(i)$ and 4.2 .5 . THEOREM 4.3 .4 . Let $\left\{u_{n}\right\} \in \mathbb{R}_{1}$. Then $u:=\sum_{n=0}^{\infty} u_{n}<\infty$ iff $\sum_{n=0}^{\infty} \frac{x_{n}(1)}{n+1}<\infty$, in
which case

$$
(4.3 .7) \quad \sum_{n=0}^{\infty} \frac{x_{n}(1)}{n+1}=\log u
$$

It is not clear how to get an analogue of theorems 4.1.2(ii) and 4.2.16, because the conditions $f=1$ and $x(\alpha)=1 /(1-\alpha)$ have no obvious analogue for $\alpha=1$. If, however, for a sequence $\left\{u_{n}\right\} \in R_{1}$ we suppose that $u_{\infty}:=\lim _{n \rightarrow \infty} u_{n}$ exists, then, using $(4.3 .6)$, we can obtain this limit as follows:

$$
\begin{aligned}
u_{\infty} & =\lim _{x \uparrow 1}(1-x) U(x)=\lim _{x \uparrow 1} \exp \left[\log (1-x)+\sum_{k=0}^{\infty} \frac{x_{k}(1)}{k+1} x^{k+1}\right]= \\
& \left.=\underset{x \uparrow 1}{\exp \left[\lim _{k=0}\right.} \sum_{k+1}^{\infty} \frac{x_{k}(1)-1}{k+1} x^{k+1}\right] .
\end{aligned}
$$


If in addition the $r_{n}(1)$ 's are such that

$$
\text { (4.3.8) } \sum_{k=0}^{\infty} \frac{r_{k}(1)-1}{k+1} \text { converges. }
$$

then by Abel's theorem it follows that

$$
(4.3 .9) \quad u_{\infty}=\exp \left[\sum_{k=0}^{\infty} \frac{r_{k}(1)-1}{k+1}\right] .
$$

Quite recently, however, Hawkes \& Jenkins (1978) proved that the absolute convergence of the series in $(4.3 .8)$ is also sufficient for the existence of $u_{\infty}=\lim _{n \rightarrow \infty} u_{n}$. In fact, they have the following results.

THEOREM 4.3.5. Let $\left\{u_{n}\right\}_{0}^{\infty}$ be an aperiodic (cf. lemma 4.3.9) sequence with $u_{0}=1$ and satisfying $(4.3 .1)$ with $r_{n}(1) \geq 0\left(n \in \mathbb{N}_{0}\right)$.

(i) If $\gamma:=\lim _{n \rightarrow \infty} r_{n}(1)$ exists in $(0, \infty)$, then $\left\{u_{n}\right\}$ satisfies $(4.3 .10) \quad u_{n} \sim \frac{n^{\gamma-1}}{\Gamma(\gamma)} \exp \left[\sum_{k=0}^{\infty} \frac{r_{k}(1)-\gamma}{k+1}\left(1-\frac{1}{n}\right)^{k+1}\right] \quad(n \rightarrow \infty)$.

(i.i) If $\sum_{k=0}^{\infty} \frac{\left|r_{k}(1)-\gamma\right|}{k+1}<\infty$ for some $\gamma \geq 1$, then (4.3.11) $u_{n} \sim \frac{n^{\gamma-1}}{\Gamma(\gamma)} \exp \left[\sum_{k=0}^{\infty} \frac{r_{k}(1)-\gamma}{k+1}\right] \quad(n \rightarrow \infty) ;$ when $\gamma=1$, then $\left\{u_{n}\right\}$ is also of bounded variation, i.e. (4.3.12) $\sum_{n=0}^{\infty}\left|u_{n+1}-u_{n}\right|<\infty$.

PROOF. For the sake of completeness we give a proof of the result that is most interesting for us, i.e. part (ii) with $\gamma=1$.

Define $d_{n}:=u_{n}-u_{n-1}\left(n \in \mathbb{N}_{0}\right)$, with $u_{-1}:=0$. Then from (4.3.1) we obtain by subtraction

$$
(4.3 .13) \quad(n+1) d_{n+1}=\sum_{k=0}^{n}\left(r_{k}(1)-1\right) d_{n-k} \quad\left(n \in \mathbb{N}_{0}\right) .
$$

Now, let $\left\{v_{n}\right\}_{0}^{\infty}$ be the sequence with $v_{0}=1$ that satisfies (4.3.1) with $r_{n}(1)$ replaced by $\bar{x}_{n}(1):=\left|r_{n}(1)-1\right|\left(n \in \mathbb{N}_{0}\right)$. Then from (4.3.13) it follows by induction that $\left|d_{n}\right| \leq v_{n}\left(n \in \mathbb{N}_{0}\right)$, and as by theorem 4.3 .4 we have 
$\Sigma v_{n}<\infty$, we conclude that $\left\{u_{n}\right\}$ is of bounded variation. Hence $\left\{u_{n}\right\}$ has a limit $u_{\infty}$, which is necessarily equal to the expression in $(4.3 .9)$.

REMARK 4.3.6. As noted by Hawkes \& Jenkins, theorem 4.3.5(i) includes the renewal theorem: if $\left\{u_{n}\right\}$ is an aperiodic renewal sequence, associated with $\left\{f_{n}\right\}$ such that $\mu:=\sum n f_{n}<\infty$, then the $r_{n}(1)^{\prime} s$, corresponding to $\left\{u_{n}\right\}$, satisfy $\lim _{n \rightarrow \infty} r_{n}(1)=1$ (cf. Port $(1964)$ ), and

(4.3.14) $\lim _{n \rightarrow \infty} \sum_{k=0}^{\infty} \frac{r_{k}(1)-1}{k+1}\left(1-\frac{1}{n}\right)^{k+1}=\frac{1}{\mu}$.

The limit theorems 4.1 .14 for $R_{0}$ and 4.2 .17 for $R_{\alpha}(0<\alpha<1)$ do have an analogue for $R_{1}$. As in the case $0 \leq \alpha<1$, we need an inequality and an observation concerning the period of $\left\{u_{n}\right\} \in R_{1}$. Since for the function $h_{n}(\alpha)$, defined in $(4.2 .17)$, we have

$$
h_{n}(1):=\lim _{\alpha \uparrow 1} h_{n}(\alpha)=n: \quad\left(n \in \mathbb{N}_{0}\right)
$$

and as the discussion in the proof of theorem 4.2 .12 also holds for $\alpha=1$, we get the following inequality for $\left\{u_{n}\right\} \in R_{1}$.

THEOREM 4.3.7. If $\left\{u_{n}\right\}_{0}^{\infty} \in R_{1}$, then

$(4.3 .15) \quad\left(\begin{array}{c}n+k \\ n\end{array}\right) u_{n+k} \geq u_{n} u_{k} \quad\left(n, k \in \mathbb{N}_{0}\right)$.

COROLLARY 4.3.8. If $\left\{u_{n}\right\} \in R_{1}$, then the following implication and inequality hold:

$$
\begin{aligned}
& \text { (4.3.16) }\left[u_{n}>0 \text { and } u_{k}>0\right] \Rightarrow u_{n+k}>0 \quad\left(n, k \in \mathbb{N}_{0}\right), \\
& \text { (4.3.17) } u_{n} \geq u_{1}^{n} / n: \quad\left(n \in \mathbb{N}_{0}\right) .
\end{aligned}
$$

We note that the inequalities in (4.3.15) and (4.3.17) become equalities for $\left\{\tilde{u}_{n}\right\} \in R_{1}$, corresponding to $\tilde{r}_{0}(1)=\mu>0, \tilde{r}_{n}(1)=0$ (n $\left.\in \mathbb{N}\right)$, i.e.

$$
(4.3 .18) \quad \widetilde{u}_{n}=\mu^{n} / n: \quad\left(n \in \mathbb{N}_{0}\right) \text {, }
$$

i.e. the $R_{1}$-analogue of the poisson distribution (cf. $\left\{\tilde{u}_{n}\right\} \in R_{\alpha}$ in remark $4.2 .3)$. 
The period of $\left\{u_{n}\right\} \in R_{1}$ has properties similar to those in the case $0 \leq \alpha<1$ (cf. theorem 4.1.14 and lemna 4.2.15; see also Wright (1967)).

LEMMA 4.3.9. Let $\left\{u_{n}\right\} \in R_{1}$ and suppose that the $r_{n}(1) ' s$, corresponding to $\left\{u_{n}\right\}$, are not all zero. Then the period $d$ of $\left\{u_{n}\right\}$ is equal to that of the sequence $\left\{x_{n-1}(1)\right\}_{1}^{\infty}$, and

$(4.3 .19) \#\left(\left\{n \in \mathbb{N} \mid u_{n d}=0\right\}\right)<\infty$.

PROOF. Let $\left\{u_{n}\right\} \in R_{1}$ with period $d$, and let $\delta$ be the period of $\left\{x_{n-1}(1)\right\}_{1}^{\infty}$. In view of $(4.3 .4)$ we have

$$
\left\{n \in \mathbb{N} \mid r_{n-1}(1)>0\right\} \subset\left\{n \in \mathbb{N} \mid u_{n}>0\right\},
$$

and hence $\delta \geq d$. Since $r_{n-1}(1)=0$ unless $\delta$ divides $n(n \in \mathbb{N})$, from $(4.3 .6)$ it is seen that $U$ is a power series in $z^{\delta}$, i.e.

$$
\left\{\mathrm{n} \in \mathbb{N} \mid \mathrm{u}_{\mathrm{n}}>0\right\} \subset\{k \delta \mid k \in \mathbb{N}\}
$$

so that $d \geq \delta$. It follows that $d=\delta$. To prove (4.3.19) we introduce the set $\mathbb{A}=\left\{k_{1}, k_{2}, \ldots\right\}$ as the set of $k \in \mathbb{N}$ for which $u_{k d}>0$. Then there exists $\mathbb{N} \in \mathbb{N}$ such that

$$
\operatorname{gcd} A=\operatorname{gcd}\left\{k_{1} \ldots, k_{N}\right\}=1
$$

It is well known that there now exists $K \in \mathbb{I N}$ such that every $k \geq k(k \in \mathbb{I N})$ can be written as

$$
k=\sum_{j=1}^{\mathbb{N}} m_{j} k_{j}
$$

where $\mathrm{m}_{j} \in \mathbb{N}(j=1, \ldots, \mathrm{N})$. By $(4.3 .15)$ it follows that for $k \geq \mathbb{K}$

(4.3.20) $\frac{(k d):}{\prod_{j=1}^{N}\left\{\left(k_{j} d\right):\right\}} u_{j k d} \geq \prod_{j=1}^{N} u_{k_{j} d}^{m_{j}}$,

which is positive, as $u_{k_{j}}>0$ for $j=1, \ldots, N$. Hence $u_{k d}>0$ for $k \geq k$.

COROLIARY 4.3.10. If $\left\{u_{n}\right\} \in R_{1}$, and if $\left\{u_{n}\right\}$ has period $d$, then also $\left\{u_{n d}\right\}_{n=0}^{\infty} \in R_{1}$. 
PROOF. In view of the preceding lemma we have

$$
u_{n}=x_{n-1}(1)=0 \text { if } n k\left\{\ell d \mid \ell \in \mathbb{N}_{0}\right\} .
$$

Define $w_{n}:=u_{n d}\left(n \in \mathbb{N}_{0}\right)$, then it follows that for $\mathrm{n} \in \mathbb{N}$ 。

$$
\begin{aligned}
(n+1) w_{n+1} & =(n+1) u_{(n+1) d}=\frac{1}{d} \sum_{j=1}^{n+1} r_{j d-1}(1) u_{(n+1-j) d}= \\
& =\sum_{k=0}^{n} \frac{1}{d} x_{(k+1) d-1}(1) w_{n-k} .
\end{aligned}
$$

Hence $\left\{w_{n}\right\} \in R_{1}$, with $r_{n}(1)$, corresponding to $\left\{w_{n}\right\}$ and denoted by $r_{n}^{(d)}(1)$, given by

$$
r_{n}^{(d)}(1)=\frac{1}{d} r_{(n+1) d-1}(1) \quad\left(n \in \mathbb{N}_{0}\right) .
$$

REMARK 4.3.11. Theorem 4.1.7(ii) yields an analogue of corollary 4.3.10 for $R_{0}$. In the case $0<\alpha<1$, however, we have (cf. the proof of theorem $4.2 .16)$ :

$$
\text { (4.3.21) }\left\{u_{n}\right\} \in R_{\alpha} \Rightarrow\left\{u_{n d}\right\} \in R_{\alpha} d
$$

We are now ready to state the following result on the asymptotic behaviour of $\left\{u_{n}\right\} \in R_{1}$. By corollary 4.3.10 we can, and will, confine ourselves to aperiodic sequences $\left\{u_{n}\right\}$.

THEOREM 4.3.12. Let $\left\{u_{n}\right\}$ be an aperiodic sequence in $R_{1}$. Then

$$
(4.3 .22) \quad \ell:=\lim _{n \rightarrow \infty} \frac{-\log u_{n}-n \log n}{n}
$$

exists in $[-\infty, \infty)$, while

(i) $l>-\infty$ iff there exists $\gamma>0$ such that $u_{n} \leq \gamma^{n} / n !\left(n \in \mathbb{N}_{0}\right)$, in which case

(4.3.23) $\lim _{n \rightarrow \infty} \frac{-\log u_{n}}{n \log n}=1$ and $\frac{-\log u_{n}}{n \log n}-1=\frac{\ell+o(1)}{\log n} \quad(n \rightarrow \infty)$;

(ii) If $\ell=-\infty$, then

(4.3.24) $\limsup _{n \rightarrow \infty} \frac{-\log u_{n}}{n \log n} \leq 1$. 
PROOF. Let $\left\{u_{n}\right\} \in R_{1}$ be aperiodic. Then, in view of (4.3.19), for $n$ sufficiently large we can define $v_{n}$ as follows:

$$
v_{n}:=-\log \left\{n: u_{n}\right\}
$$

On account of theorem 4.3.7, $\left\{v_{n}\right\}$ is subadditive, i.e. $v_{n+k} \leq v_{n}+v_{k}$ from which (cf. Polya \& szego (1970), I. Abschn., Kap. 3) it follows that $\lim _{n \rightarrow \infty} \frac{1}{n} v_{n}$ exists in $[-\infty, \infty)$ and is equal to $l^{\prime}:=\inf \frac{1}{n} v_{n}$. As $n \log n-\log n: \sim n(n \rightarrow \infty)$, it is seen that also

$$
\begin{aligned}
\ell: & =\lim _{n \rightarrow \infty} \frac{-\log u_{n}-n \log n}{n}=\lim _{n \rightarrow \infty}\left\{\frac{1}{n} v_{n}-\frac{1}{n}(n \log n-\log n:)\right\}= \\
& =\ell^{8}-1
\end{aligned}
$$

exists in $[-\infty, \infty)$. Furthermore, if $\ell>-\infty$, then we have for $n$ sufficiently large

$$
-\frac{1}{n} \log \left\{n: u_{n}\right\} \geq \ell=\ell+1
$$

and hence $u_{n} \leq \gamma^{n} / n$ : ( $n \in \mathbb{N}_{0}$ ) for some $\gamma>0$. It is easily seen that the converse is also true, and that in this case (4.3.23) follows from (4.3.22). Finally, if $l=-\infty$, then for all sufficiently large $n$ we have $-\log u_{n}-n \log n<0$, and hence $(4.3 .24)$.

It can be proved that for an aperiodic $\left\{u_{n}\right\} \in R_{1}$ the limit $\lim _{n \rightarrow \infty}-\log u_{n} /\{n \log n\}$ always exists (cf. $(4.3 .23)$ and $(4.3 .24)$ ), and its value can be calculated. This has been done by steutel \& wolfe (1977) for $\left\{u_{n}\right\} \in C_{1}$ with $u_{1}>0$. It is easily shown, however, that their proof also holds for a general aperiodic $\left\{u_{n}\right\} \in C_{1}$. Finally, as for every $\left\{u_{n}\right\} \in R_{1}$ there exists $\gamma>0$ such that $\left\{u_{n} \gamma^{n} / u(\gamma)\right\} \in C_{1}$, we get the following result.

THEOREM 4.3.13. Let $\left\{u_{n}\right\}$ be an aperiodic sequence in $R_{1}$. Then

$$
\text { (4.3.25) } \lim _{n \rightarrow \infty} \frac{-\log u_{n}}{n \log n}=\frac{1}{N} \text {, }
$$

where $N$ is the smallest integer (possibly infinite) such that $r_{n}(1)=0$ for all $n \geq N$.

Combining this result with theorem 4.3 .12 , we obtain the following corollaries (note that $N=1$ (cf. theorem 4.3.13) iff $u_{n}=\tilde{u}_{n}$ given by (4.3.18)). 
COROLLARY 4.3.14. Let $\left\{u_{n}\right\}$ be an aperiodic sequence in $R_{1}$. Then the limit $\ell$ in $(4.3 .22)$ is finite iff there exists $\mu>0$ such that

$$
u_{n}=\mu / n: \quad\left(n \in \mathbb{N}_{0}\right)
$$

in which case necessarily $l=-\log \mu-1$.

COROLLARY 4.3.15. Let $\left\{u_{n}\right\}$ be an aperiodic sequence in $R_{1}$. If $u_{n} \leq \gamma^{n} / n$ ! $\left(n \in \mathbb{N}_{0}\right.$ ) for some $\gamma>0$, then there exists $\mu>0$ such that $u_{n}=\mu^{n} / n$ : $\left(n \in \mathbb{N}_{0}\right)$.

As $C_{\alpha} \subset C_{1}$, we have also

(4.3.26) $\quad R_{\alpha} \subset R_{1} \quad(0 \leq \alpha<1)$.

Now, if $0 \leq \alpha<1$ and if $\left\{u_{n}\right\}$ is an aperiodic sequence in $R_{\alpha}$, then by theorem 4.2 .17

$$
\lim _{n \rightarrow \infty} \frac{-\log u_{n}}{n}=-\log \theta \in[0, \infty) .
$$

It follows that the limit in $(4.3 .25)$ is zero, i.e. we have the following result.

THEOREM 4.3.16. Let $0 \leq \alpha<1$ and let $\left\{u_{n}\right\}$ be an aperiodic sequence in $R_{\alpha}$. Then the $r_{n}(1)^{\prime} \mathrm{s}$, corresponding to $\left\{u_{n}\right\}$, satisfy

$$
\forall_{N \in \mathbb{N}} \exists_{n \geq N} r_{n}(1)>0
$$




\section{CHAPTER 5}

\section{CLASSIFICATION OF THE INFINITELY DIVISIBLE DISTRIBUTIONS ON $[0, \infty$}

The first aim of this chapter is to extend the classification of $C_{1}$, defined by the classes $C_{\alpha}$ (cf, chapter 2), to all inf div distributions on $[0, \infty$ ). Here the difficulty arises that no analogue of $C_{0}$ is known for general de's on $[0, \infty)$, but generalizing a functional equation, by means of which the inf div de's on $[0, \infty$ ) are characterized (cf. theorem 1.6.2), we obtain classes $F_{\lambda}(0 \leq \lambda<\infty)$, which, by putting $\alpha=e^{-\lambda}$, can be considered to be extensions of the classes $C_{\alpha}(0<\alpha \leq 1)$. This is done in section 1 , which also contains some preliminary results. These are necessary, because the proofs of the monotonicity of $F_{\lambda}$ (section 2) and further properties (section 3) turn out to be more delicate than in the discrete case. This is mainly due to the necessity of considering also distributions on $[0, \infty)$ without a jump at zero, as will be apparent from the proofs, which will only be given as far as they are essentially different from those in the discrete case. In section 4 we study the limiting class $F_{\infty}:=\lim _{\lambda \rightarrow \infty} F_{\lambda}$. In many respects, this class turns out to be the analogue of $C_{0}$ for de's on $[0, \infty)$. For instance, the df"s $F^{\prime}$ in $F_{\infty}$ with $F(O)>0$ corxespond with the compound geometric df: $s$ on $[0, \infty)$, and $F_{\infty}$ contains the log-convex, and hence the comp mon, densities on $(0, \infty)$ (cf. the end of section 1.6). Furthermore, we show that the PLST"s of df's in $F_{\infty}$ have a canonical representation that is very similax to that of the LT's of continuous analogues of the renewal sequences (class $R_{0}$ ), the standard p-functions (class $P$ ) of Kingman (1972). The re-sulting relation between $P$ and (a part of) $F_{\infty}$ is considered in section 5. Many properties of $P$, often easily obtained by the probabilistic interpretation of a p-function, can be translated for the corresponding densities in $F_{\infty}$. Also, the af's in $F$ have interesting relations with the renewal functions (cf. Smith (1958)) and the potential kernels (cf. Berg \& Forst (1975) and Hawkes (1977)). However, we shall not investigate these relations in detail in this monograph.

In section 6 we show that two different interpolations between $F_{\infty}$ and $F_{0}$ give both rise to classes of df's that are limits of compound negative-binomial df"s on $[0, \infty)$ (cf. section 2.5). These classes also define a classification of $F_{0}$

Finally, in section 7 we mention some further generalizations. Specifically, we briefly discuss the classification of inf div distributions on $\mathrm{IR}$ and on $[0, \infty)^{2}$ by means of Eunctional equations. 


\subsection{The classes $F_{\lambda}$; preliminaries}

In chapter 2 the classification of $C_{1}$, defined by the classes $C_{\alpha}(0 \leq \alpha \leq 1)$, was achieved by generalizing the recurrence relations by means of which $C_{1}$ and $C_{0}$ are characterized, $i . e$. the relations for a lattice distribution $\left\{p_{n}\right\}_{0}^{\infty}$ given by

$$
(5.1 .1) \quad(n+1) p_{n+1}=\sum_{k=0}^{n} p_{k}{ }_{n-k}(1) \quad\left(n \in \mathbb{N}_{0}\right)
$$

and

$$
\text { (5.1.2) } \quad p_{n+1}=\sum_{k=0}^{n} p_{k} r_{n-k}(0) \quad\left(n \in \mathbb{N}_{0}\right)
$$

respectively. Now we want to give a similar classification of the class of all inf div distributions on $[0, \infty)$. The starting point for this is the analogue of (5.1.1) for densities (cf. corollary 1.6.3): a pdf $f$ on $(0, \infty)$ is inf div iff there exists a right--continuous, nondecreasing function $k_{0}$ such that

$$
\text { (5.1.3) } x f(x)=\int_{[0, x]} f(x-y) d K_{0}(y) \quad \text { (almost all } x>0 \text { ). }
$$

For pdf"s there is no obvious analogue of $(5.1 .2)$; this would be

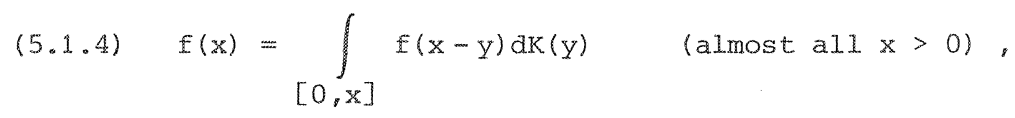

with a right-continuous, nondecreasing function $\mathrm{K}$. However, (5.1.4) is satisfied by all pdf's if $\mathrm{K}$ is the unit-step function at zero and by none for any other $\mathrm{K}$. To overcome this difficulty we first generalize the classes $C_{\alpha}$ for $\alpha>0$. The set of (absolutely continuous) distributions in the intersection of the resulting classes will then be considered as the analogue of $C_{0}$ for (absolutely continuous) distributions on $[0, \infty)$.

Proceeding as in the discrete case we replace the factor $x$ in the left--hand side of $(5.1 .3)$ by the function

$$
(5.1 .5) \quad c(x ; \lambda):=\left(1-e^{-\lambda x}\right) /\left(1-e^{-\lambda}\right) \quad(0<\lambda<\infty ; x \geq 0),
$$

with $c(x ; 0):=x(x \geq 0)$. This function is obtained from $c_{n}(\alpha)=\left(1-\alpha^{n+1}\right) /$ $(1-\alpha)$ in $(2.3 .1)$ by replacing $\alpha$ with $e^{-\lambda}$; in fact, we have

$$
\text { (5.1.6) } \quad c_{n}(\alpha)=c(n+1 ;-\log \alpha) \quad\left(n \in \mathbb{N}_{0} ; 0<\alpha \leq 1\right)
$$


and, as in the discrete case, we define for $\lambda>0$

$$
c_{\lambda}:=\lim _{x \rightarrow \infty} c(x ; \lambda)=\left(1-e^{-\lambda}\right)^{-1}
$$

Considering, more generally, df's rather than pdf's, we are led to the following analogues of the classes $C_{\alpha}$ for $0<\alpha \leq 1$.

DEFINITION 5.1.1. For $0 \leq \lambda<\infty$ a df $F$ on $[0, \infty)$ is said to be in the class $F_{\lambda}$ if there exists a right-continuous, nondecreasing function $K_{\lambda}$, vanishing on $(-\infty, 0)$, such that

$$
(5.1 .7) \quad \int_{[0, x]} c(y ; \lambda) d F(y)=\int_{[0, x]} F(x-y) d k_{\lambda}(y) \quad(x \geq 0) .
$$

If $F \in F_{\lambda}$, then we shall also say that $\hat{F} \in F_{\lambda}$; similarly, $f \in F_{\lambda}$ if $F \in F_{\lambda}$ has density $f$. As we shall see from its LST, the function $k_{\lambda}$ in definition 5.1 .1 is uniquely determined by $F \in F_{\lambda}$; it will be called the $k_{\lambda}$-function of $\mathrm{F} \in F_{\lambda}$.

It is convenient to introduce the following (disjoint) subclasses of $F_{\lambda}$ for $0 \leq \lambda<\infty$ :

$$
\begin{aligned}
F_{\lambda}^{+} & :=\left\{F \in F_{\lambda} \mid F(0)>0\right\} \\
F_{\lambda}^{\prime} & :=\left\{F \in F_{\lambda} \mid F \text { is absolutely continuous }\right\} .
\end{aligned}
$$

The classes $F_{\lambda}^{+}$contain the classes $C_{\alpha}$; in fact, for $0<\alpha \leq 1$ we have

$$
\text { (5.1.9) } \quad C_{\alpha}=\left\{F \in F_{-\log \alpha}^{+} \mid F \text { is concentrated on } \mathbb{I N}_{0}\right\} .
$$

This easily follows by showing that if $F$ is the df corresponding to a distribution $\left\{p_{n}\right\}_{0}^{\infty}$ on $\mathbb{N}_{0}$ with $p_{0}>0$ and if $\lambda=-\log \alpha$, then the recurrence relations (2.3.1) and the functional equation (5.1.7) are equivalent: use $(5.1 .6)$ and the following relation between the $\mathrm{K}_{\lambda}$-function of $\mathrm{F}$ and the $r_{n}(\alpha)$ 's corresponding to $\left\{p_{n}\right\}$ (cf. theorem 1.7.7):

$$
K_{\lambda}(x)=\sum_{n=1}^{\infty} x_{n-1}(\alpha) 1_{[n, \infty)}(x) \quad(x \in \mathbb{R}) .
$$

The classes $F_{\lambda}^{\prime}$ can be characterized as follows (cf. corollary 1.6.3).

THEOREM 5.1.2. For $0 \leq \lambda<\infty$ a paf $f$ on $(0, \infty)$ is in $F_{\lambda}^{3}$ iff there exists a right-continuous, nondecreasing function $k_{\lambda}$ such that 
$(5.1 .10) \quad c(x ; \lambda) f(x)=\int_{[0, x]} f(x-y) d K_{\lambda}(y) \quad$ (almost all $\left.x>0\right)$.

PROOF. If $F$ is a df on $[0, \infty)$ with pdf $f$, then for all $x>0$ we have, on the one hand

$$
\int_{[0, x]} c(y ; \lambda) d F(y)=\int_{[0, x]} c(y ; \lambda) f(y) d y,
$$

and on the other hand (using Fubini's theorem)

$$
\begin{aligned}
& \int_{[0, x]} F^{2}(x-y) d K_{\lambda}(y)=\int_{[0, x]} \int_{[0, x-y]} f(u) d u d K_{\lambda}(y)= \\
& =\int_{[0, x]} \int_{[y, x]} f(v-y) d v d K_{\lambda}(y)=\int_{[0, x][0, v]} f(v-y) d K_{\lambda}(y) d v .
\end{aligned}
$$

Now, in view of $(5.1 .7)$, the theorem easily follows.

Taking $\lambda=0$ in definition 5.1.1, from theorem 1.6 .2 one sees that $F_{0}$ is the class of all inf div df's on $[0, \infty)$. Furthermore, on account of theorem 1.6.6 it follows that $F_{0}^{+}$is the class of all compound Poisson distributions on $[0, \infty)$. To obtain the compound geometric distributions on $[0, \infty)$, we formally let $\lambda \rightarrow \infty$ in $(5.1 .7)$. As

$$
c(x ; \infty):=\lim _{\lambda \rightarrow \infty} c(x ; \lambda)= \begin{cases}0 & \text { if } x=0, \\ 1 & \text { if } x>0,\end{cases}
$$

we get

$(5.1 .11) \quad F(x)-F(0)=\int_{[0, x]} F(x-y) d K_{\infty}(y) \quad(x \geq 0)$.

If $F(0)=0$, the same difficulty axises as in (5.1.4) for paf's, but if $F(0)>0,(5.1 .11)$ makes sense; the df's $F$ on $[0, \infty)$, with $F(0)>0$ and satisfying (5.1.11) with a right-continuous, nondecreasing function $\mathrm{K}_{\infty}$, correspond to the compound geometric distributions on $[0, \infty)$ (cf. theorem 1.6.7). This class of distributions we denote by $F_{\infty}^{+}(\mathrm{cf} .(5.1 .8))$, since it turns out to be the subset of df's $F$ with $F(0)>0$ in a class $F_{\infty}$ to be defined later. Thus we have the following definition:

(5.1.12) $F_{\infty}^{+}:=\{$df $F$ on $[0, \infty) \mid F$ is compound geometric $\}$. 
We require that the function $K_{\infty}$ in $(5.1 .11)$ vanishes on $(-\infty, 0)$. It is then uniquely determined by $F \in F_{\infty}^{+}$, and will be called the $K_{\infty}$-function of $F \in F_{\infty}^{+}$.

In order to obtain a characterization of $F_{\lambda}(0<\lambda<\infty)$ and $F_{\infty}^{+}$, analogous to that of $F_{0}$ given in the first part of theorem 1.6.1, we take LST's in $(5.1 .7)$ and $(5.1 .11)$ to obtain

(5.1.13) $\quad c_{\lambda}\{\hat{F}(\tau)-\hat{F}(\tau+\lambda)\}=\hat{F}(\tau) \hat{K}_{\lambda}(\tau) \quad(\tau>0)$, and

$(5.1 .14) \quad \hat{F}(\tau)-F(0)=\hat{F}(\tau) \hat{K}_{\infty}(\tau) \quad(\tau>0)$.

Now, for an arbitrary df $F$ on $[0, \infty)$, we define the $\varphi_{\lambda}$-function of $F$ as the solution of $(5.1 .13)$ or $(5.1 .14)$ for $\hat{\mathrm{K}}_{\lambda}$, i.e. we have the following definition.

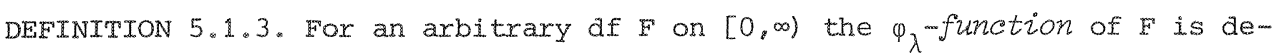
fined by

$$
\begin{aligned}
& \varphi_{\lambda}(\tau):=c_{\lambda}\{1-\hat{F}(\tau+\lambda) / \hat{F}(\tau)\} \quad \text { if } 0<\lambda<\infty, \\
& \varphi_{0}(\tau):=\lim _{\lambda \downarrow 0} \varphi_{\lambda}(\tau)=-\hat{F}^{\prime}(\tau) / \hat{F}(\tau)=-\frac{d}{d \tau} \log \hat{F}(\tau),
\end{aligned}
$$

and, if $F(0)>0$,

$$
\varphi_{\infty}(\tau):=\lim _{\lambda \rightarrow \infty} \varphi_{\lambda}(\tau)=1-F(0) / \hat{F}(\tau) .
$$

We note that for $0 \leq \lambda<\infty$ the $\varphi_{\lambda}$-function of a df $F \in F_{\lambda}$ coincides with the LST of the $K_{\lambda}$-function of $F, i . e, \varphi_{\lambda}=\hat{K}_{\lambda}$. Similarly $\varphi_{\infty}=\hat{K}_{\infty}$, if $F \in F_{\infty}^{+}$. By Bernstein's theorem (theorem 1.3.7) we immediately obtain the following charactexization of $F_{\lambda}$ and $F_{\infty}^{+}$(cf. lemma 2.3.3).

LEMMA 5.1 .4 .

(i) For $0 \leq \lambda<\infty$ a df $F$ on $[0, \infty)$ is in $F_{\lambda}$ iff its $\varphi_{\lambda}$-function is compmon. (ii) $A$ df $F$ on $[0, \infty)$ with $F(0)>0$ is in $F_{\infty}^{+}$iff its $\varphi_{\infty}$-function is compmon.

We shall need the limiting behaviour for $\tau \rightarrow \infty$ of the $\varphi_{\lambda}$-functions of a df $F$. For $\lambda>0$ this is obtained from the following lemma, which does not seem to be generally known (Cf. Van Harn (1977)). The quantity $\ell(F)$ is the left extremity of F (cf. section 1.2). 
138

LEMMA 5.1.5. If $a>0$ and if $F$ is $a$ df on $[0, \infty)$, then the function $\hat{\mathrm{F}}(\tau+\mathrm{a}) / \hat{\mathrm{F}}(\tau)$ is nondecreasing, and satisfies

(5.1.15) $\lim _{\tau \rightarrow \infty} \hat{\mathrm{F}}(\tau+a) / \hat{\mathrm{F}}(\tau)=\exp [-a \ell(F)]$.

PROOF. Let $a>0$ and let $F$ be a df on $[0, \infty)$. As by Bernstein's theorem $\hat{\mathrm{F}}$ is comp mon, it is log-convex (use Schwarz's inequality; see also theorem 1.6.11), i.e. $\log \hat{F}(\tau)$ is convex. It follows that the $\varphi_{0}$-function $\varphi_{0}$ of $F$ is nonincreasing, and as

$(5.1 .16) \quad \frac{d}{d \tau}\{\hat{F}(\tau+a) / \hat{F}(\tau)\}=\left\{\varphi_{0}(\tau)-\varphi_{0}(\tau+a)\right\} \hat{F}(\tau+a) / \hat{F}(\tau)$

it is seen that the function $\hat{F}(\tau+a) / \hat{F}(\tau)$, which takes values in $(0,1]$, is nondecreasing, and hence has a limit in $(0,1]$ as $\tau \rightarrow \infty$.

The proof of $(5.1 .15$ ) we give here, is due to $\mathrm{W}$. Vervaat (see also remark 5.1.7). First, take $\ell(F)=0$. Then for $(5.1 .15)$ it is sufficient to prove that

(5.1.17) $\liminf \hat{F}(\tau+a) / \hat{F}(\tau) \geq 1$. $\tau \rightarrow \infty$

For every $\tau>0$ and $\varepsilon>0$

$$
\hat{F}(\tau+a) \geq e^{-a \varepsilon} \int_{[0, \varepsilon]} e^{-\tau x} d F(x) \geq e^{-a \varepsilon}\left\{\hat{F}(\tau)-e^{-\tau \varepsilon}\right\} .
$$

Since

$$
0 \leq e^{-\tau \varepsilon} \hat{F}(\tau)^{-1} \leq e^{-\tau \varepsilon}\left\{\int_{[0, \varepsilon / 2]} e^{-\tau x} d F(x)\right\}^{-1} \leq e^{-\tau \varepsilon / 2} F(\varepsilon / 2)^{-1},
$$

it follows that for all $\varepsilon>0$

$$
\underset{\tau \rightarrow \infty}{\liminf } \hat{F}(\tau+a) / \hat{F}(\tau) \geq e^{-a \varepsilon}
$$

which yields (5.1.17), and hence (5.1.15) with $\ell(F)=0$.

Finally, using this result for the df $G(x):=F(x+l(F))$, we easily obtain $(5.1 .15)$ for an arbitrary df $\mathrm{F}$ on $[0, \infty)$

LEMMA 5.1.6. If $0 \leq \lambda \leq \infty$ and if $F$ is a df on $[0, \infty)$ (with $F(0)>0$ if $\lambda=\infty$ ), then the $\varphi_{\lambda}$-function of $\mathrm{F}$ satisfies (Cf. (5.1.5))

(5.1.18) $\lim _{\tau \rightarrow \infty} \varphi_{\lambda}(\tau)=c(\ell(F) ; \lambda)$. 
PROOF. The result follows from $(5.1 .15)$ if $0<\lambda<\infty$, and is trivial if $\lambda=\infty$. To prove $(5.1 .18)$ for $\lambda=0$, we may restrict ourselves to df's $F$ with $\ell(F)=0$ (Cf. the proof of lemma 5.1.5). Then using the identity

$$
\hat{F}(\tau)=\tau \int_{(0, \infty)} e^{-\tau x} F(x) d x \quad(\tau>0) .
$$

we obtain for $\varepsilon>0$

$$
\begin{aligned}
\varphi_{0}(\tau) & =-\hat{F}^{\prime}(\tau) / \hat{F}(\tau) \leq\left\{\int_{(0, \infty)} e^{\left.-\tau x_{F}(x) d x\right\}^{-1}} \int_{(0, \infty)} x e^{-\tau x} F(x) d x \leq\right. \\
& \leq \varepsilon+\left\{F(\varepsilon / 2) \int_{(\varepsilon / 2, \infty)} e^{-\tau x} d x\right\}^{-1} \int_{(\varepsilon, \infty)} x e^{-\top \tau x} d x= \\
& =\varepsilon+F(\varepsilon / 2)^{-1}\{\varepsilon+1 / \tau\} \exp [-\tau \varepsilon / 2],
\end{aligned}
$$

which is less than $2 \varepsilon$ for $\tau$ sufficiently large. Hence $\lim _{\tau \rightarrow \infty} \varphi_{0}(\tau)=0$.

REMARK 5.1.7. Since for all $\tau>0$ there exists $\theta(\tau) \in(0,1)$ such that

$$
\log \{\hat{F}(\tau+a) / \hat{F}(\tau)\}=-a \varphi_{0}(\tau+\theta(\tau) a),
$$

(5.1.15) also immediately follows from $(5.1 .18)$ with $\lambda=0$.

In (1.6.2) and lemma 1.6.4 some properties of the $k_{0}$-function (i.e. the canonical function) of an inf div af on $[0, \infty)$ are given. The $k_{\lambda}$-function of a df in $F_{\lambda}$ has analogous properties. In the following lemma we prove two of them by considering the LST $\hat{K}_{\lambda}$ of $\mathrm{K}_{\lambda}$, but they can also be obtained from the functional equations (5.1.7) and (5.1.11). A third property of $k_{\lambda}$ will be given in lemma 5.2 .2 .

LEMMA 5.1.8. If $0<\lambda \leq \infty$ and if $F \in F_{\lambda}$ (with $F(0)>0$ if $\lambda=\infty$ ), then the $\mathrm{K}_{\lambda}$-function of $\mathrm{F}$ satisfies

$$
(5.1 .19) \quad K_{\lambda}(0)=c(\ell(F) ; \lambda)
$$

and

$(5.1 .20) \quad \mathrm{K}_{\lambda}(\infty)=\int_{[0, \infty)} \mathrm{dK}_{\lambda}(x)=\mathrm{c}_{\lambda}\{1-\hat{\mathrm{F}}(\lambda)\}<\mathrm{c}_{\lambda}$. 
PROOF. If $F \in F_{\lambda}$, then $\hat{\mathrm{K}}_{\lambda}$ coincides with the $\varphi_{\lambda}$-function of $F$, for which we have lemma 5.1 .6 . Now from $(1.3 .5)$ it follows that

$$
\mathrm{K}_{\lambda}(0)=\lim _{\tau \rightarrow \infty} \hat{\mathrm{K}}_{\lambda}(\tau)=\mathrm{c}(\ell(\mathrm{F}) ; \lambda)
$$

and from $(1.3 .6)$

$$
K_{\lambda}(\infty)=\lim _{\tau \downarrow 0} \hat{K}_{\lambda}(\tau)=\lim _{\tau \downarrow 0} c_{\lambda}\{1-\hat{F}(\tau+\lambda) / \hat{F}(\tau)\}=c_{\lambda}\{1-\hat{F}(\lambda)\} .
$$

Finally, we state a property of $F_{0}$ that we need in the next section to prove the monotonicity of $F_{\lambda}$.

THEOREM 5.1.9. If $F \in F_{0}$ and $a>0$, then the function $\varphi$, defined by

$$
\varphi(\tau):=\hat{F}(a) \hat{F}(\tau) \hat{F}(\tau+a) \quad(\tau \geq 0),
$$

is a PLST in $\mathrm{F}_{0}^{+}$.

PROOF。 Let $F \in F_{0}$ with canonical function $K_{0}$. In view of theorem 1.6 .1 we calculate

$$
-\frac{d}{d \tau} \log \varphi(\tau)=\hat{K}_{0}(\tau)-\hat{K}_{0}(\tau+a),
$$

which is a comp mon function. As furthermore $\varphi(0)=1$, it follows that $\varphi$ is the PLST of a df $\mathrm{F}_{a}$ in $F_{O^{\prime}}$ for which by lemma 5.1 .5 we have

$$
F_{a}(0)=\lim _{\tau \rightarrow \infty} \varphi(\tau)=\hat{F}(a) \exp [a l(F)]>0 .
$$

\subsection{The monotonicity of $F_{\lambda}$, absolute continuity}

In this section we show that the classes $F_{\lambda}$ define a classification of the inf div df's on $[0, \infty), \dot{i} . e$. we show that $F_{\lambda}$ depends monotonically on $\lambda$. To this end it is convenient to consider only df's $F$ for which

$$
(5.2 .1) \quad l(F)=0 \text { : }
$$

this is not an essential restriction (cf. theorem 5.3.2(i)). Now, for instance, it follows that the $\varphi_{\lambda}$-function of $F$ satisfies $\lim _{\tau \rightarrow \infty} \varphi_{\lambda}(\tau)=0$ (cf. lemma 5.1.6), and, if $F \in F_{\lambda}$, that $\mathrm{K}_{\lambda}(0)=0$ (cf. (5.1.19)). First we prove that all distributions in the classes $F_{\lambda}$ with $0<\lambda<\infty$ are inf div. 
THEOREM 5.2.1. For all $\lambda \in(0, \infty)$ the following inclusion holds: $F_{\lambda} \subset F_{0}$.

PROOF. We use the method of proof of theorem 2.3.4. It will then be clear that by iteration of $(5.1 .13) \hat{\mathrm{F}}$ can be written as

$$
\hat{F}(\tau)=\frac{\hat{F}(\tau+n \lambda)}{\hat{F}(n \lambda)} \prod_{k=0}^{n-1} \frac{1-c_{\lambda}^{-1} \hat{K}_{\lambda}(k \lambda)}{1-c_{\lambda}^{-1} \hat{K}_{\lambda}(\tau+k \lambda)} \quad(\tau \geq 0 ; n \in \mathbb{N}) .
$$

Now using the fact that $\hat{F}(\tau+n \lambda) / \hat{F}(n \lambda)$ tends to 1 as $n \rightarrow \infty$ (cf. lemma 5.1.5), we obtain the following expression for $\hat{\mathrm{F}}$ in its $\mathrm{K}_{\lambda}$-function:

(5.2.2) $\hat{\mathrm{F}}(\tau)=\prod_{k=0}^{\infty} \frac{1-\mathrm{c}_{\lambda}^{-1} \hat{\mathrm{K}}_{\lambda}(\mathrm{k} \lambda)}{1-\mathrm{c}_{\lambda}^{-1} \hat{\mathrm{K}}_{\lambda}(\tau+k \lambda)} \quad(\tau \geq 0)$,

from which, as in the discrete case, it follows that $F$ is inf div.

From (5.2.2) we obtain a characterization of $F_{\lambda}^{+}$for $0<\lambda<\infty$ in terms of the $\mathrm{K}_{\lambda}$-function. It can be considered as an analogue of lemma 1.6.4(ii), where $\mathrm{F}_{0}^{+}$is characterized similarly.

LEMMA 5.2.2. Let $0<\lambda<\infty$ and let $F \in F_{\lambda}$. Then $F \in F_{\lambda^{\prime}}^{+}$i.e. $F(0)>0$, iff the $\mathrm{K}_{\lambda}$-function of $\mathrm{F}$ satisfies

$(5.2 .3) \quad \int_{(0, \infty)} c(x ; \lambda)^{-1} d K_{\lambda}(x)<\infty$.

in which case

$(5.2 .4) \quad F(0)=\prod_{k=0}^{\infty}\left\{1-c_{\lambda}^{-1} \hat{K}_{\lambda}(k \lambda)\right\}$.

PROOF. Let $F \in F_{\lambda}$. Then (5.2.2) holds, from which, taking $\tau=n \lambda$, we see that

(5.2.5) $\hat{F}(n \lambda)=\prod_{k=0}^{n-1}\left\{1-c_{\lambda}^{-1} \hat{K}_{\lambda}(k \lambda)\right\} \quad(n \in \mathbb{N})$.

As $F(0)=\lim _{n \rightarrow \infty} \hat{F}(n \lambda)$, from $(5.2 .5)$ it follows that $F(0)>0$ iff the infinite product in (5.2.4) converges, or, equivalently, iff 
$(5.2 .6) \quad \sum_{k=0}^{\infty} \hat{\mathrm{K}}_{\lambda}(k \lambda)<\infty$.

Since by $(5.2 .1)$ we have $\mathrm{K}_{\lambda}(0)=0$, we can write, using Fubini.'s theorem,

$$
c_{\lambda}^{-1} \sum_{k=0}^{\infty} \hat{K}_{\lambda}(k \lambda)=c_{\lambda}^{-1} \int_{(0, \infty)} \sum_{k=0}^{\infty}\left\{e^{-\lambda x}\right\}^{k} d K_{\lambda}(x)=\int_{(0, \infty)} c(x ; \lambda)^{-1} d K_{\lambda}(x),
$$

and the lemma is proved.

For the classes $F_{O^{\prime}} F_{0}^{+}$and $F_{\infty}^{+}$we have already representation theorems (cf. theorems $1.6 .1,1.6 .6$ and 1.6 .7 , respectively). Now, from (5.2.2) we easily obtain the following representation for PLST's in $F_{\lambda}$.

THEOREM 5.2.3. For $0<\lambda<\infty$ a df $F$ (with $\ell(F)=0$ ) is in $F_{\lambda}$ iff $\hat{F}$ has the form

$(5.2 .7) \quad \hat{F}(\tau)=\prod_{k=0}^{\infty} \frac{1-\hat{p} \hat{G}(k \lambda)}{1-p \hat{G}(\tau+k \lambda)} \quad(\tau \geq 0)$,

where $0 \leq p<1$ and $G$ is a df with $G(0)=0$. The representation $(p, G)$ is unique.

PROOF. Apply the method of proof of theorem 2.3.5, and use the properties of the $\mathrm{K}_{\lambda}$-function of $\mathrm{F}$ from lemma 5.1.8.

Next we turn to the general monotonicity property. Once it has been proved for $F$, it also follows for $F^{*}, F^{+}$and $C$, and for every other set of classes, obtained from the $F_{\lambda}$ 's by intersecting them with a set of df's that does not depend on $\lambda$. We think it useful to give a full proof, although the first part is analogous to the proof of theorem 2.3.7.

THEOREM 5.2.4. For all $\lambda \in[0, \infty)$ and $\mu \in[0, \infty)$ the following inclusion holds:

$$
F_{\lambda} \subset F_{\mu} \quad \text { if } \lambda \geq \mu \text {. }
$$

PROOF. The theorem has been already proved in the case $\lambda \in[0, \infty), \mu=0$. So, in view of lemma 5.1.4(i), we have to show that, if $0<\mu \leq \lambda<\infty$ and if the $\varphi_{\lambda}$-function of a df $F$ on $[0, \infty)$ is comp mon, then its $\varphi_{\mu}$-function is comp mon. Now, according to definition 5.1.3, the $\varphi_{\mu}$-function of $F$ satisfies 
(5.2.8) $\varphi_{\mu}(\tau)-\varphi_{\mu}(\tau+\lambda)=c_{\mu}\{\hat{F}(\tau+\lambda+\mu) / \hat{F}(\tau+\lambda)-\hat{F}(\tau+\mu) / \hat{F}(\tau)\}$.

If the right-hand side of $(5.2 .8)$ is divided by $c_{\mu} \hat{F}(\tau+\mu)$, it becomes symmetric in $\lambda$ and $\mu$, and so

(5.2.9) $\quad c_{\lambda}\left\{\varphi_{\mu}(\tau)-\varphi_{\mu}(\tau+\lambda)\right\}=\frac{\hat{F}(\tau+\mu)}{\hat{F}(\tau+\lambda)} c_{\mu}\left\{\varphi_{\lambda}(\tau)-\varphi_{\lambda}(\tau+\mu)\right\}$.

If $\varphi_{\lambda}$ is comp mon, then $\varphi_{\lambda}(\tau)-\varphi_{\lambda}(\tau+\mu)$ is comp mon too. Further, as $F \in F_{\lambda} \subset F_{0}$ (theorem 5.2.1) and as $\mu \leq \lambda$, from theorem 5.1.9 it follows that the function $\hat{F}(\tau) / \hat{F}(\tau+\lambda-\mu)$, and hence $\hat{F}(\tau+\mu) / \hat{F}(\tau+\lambda)$, is comp mon. Thus, from (5.2.9) we conclude that $\varphi_{\mu}(\tau)-\varphi_{\mu}(\tau+\lambda)$ is a comp mon function. Now, using the fact that $\lim _{n \rightarrow \infty} \varphi_{\mu}(\tau+n \lambda)=0$ (cf. lemma 5.1.6), for $\varphi_{\mu}$ we can
write

$$
\varphi_{\mu}(\tau)=\sum_{k=0}^{\infty}\left\{\varphi_{\mu}(\tau+k \lambda)-\varphi_{\mu}(\tau+k \lambda+\lambda)\right\} .
$$

It follows that $\varphi_{\mu}$ is the limit of a sequence of sums of comp mon functions. Hence $\varphi_{\mu}$ is comp mon, and the theorem is proved.

By letting $\lambda \rightarrow \infty$ in $(5.2 .9)$ we see that if $\varphi_{\infty}$ is comp mon, then $\varphi_{\mu}$ is comp mon for all $\mu \in(0, \infty)$, so (cf. lemma 5.1.4(ii)) we have $F_{\infty}^{+} \subset \cap \overbrace{\lambda<\infty} F_{\lambda}^{+}$. On the other hand $\varphi_{\infty}=\lim _{\lambda \rightarrow \infty} \varphi_{\lambda}$ is comp mon, if all $\varphi_{\lambda}$ are, and so

$(5.2 .10) \quad F_{\infty}^{+}=\bigcap_{\lambda<\infty} F_{\lambda}^{+}$

This allows us to define the classes $F_{\infty}$ and $F_{\infty}^{\prime}$ as follows (cf. (5.1.8) and $(5.1 .12)):$$$
F_{\infty}:=\prod_{\lambda<\infty} F_{\lambda}=\lim _{\lambda \rightarrow \infty} F_{\lambda} .
$$$$
F_{\infty}^{\prime}:=\left\{F \in F_{\infty} \mid F \text { is absolutely continuous }\right\} \text {. }
$$

of course, theorem 5.2 .4 can now be supplemented with the case $\lambda=\infty$, $\mu \in[0, \infty]$.

If $F \in F_{\lambda}$, then theorem 5.2 .4 ensures the existence of the $K_{\mu}$-functions of F for all $\mu \in[0, \lambda]$. From (5.2.9) we obtain the following properties of these functions. 
THEOREM 5.2.5. Let $0<\lambda \leq \infty$ and let $F \in F_{\lambda}$ (with $F(0)>0$ if $\lambda=\infty$ ) with an absolutely continuous $k_{\lambda}$-function (density $k_{\lambda}$ ). Then for all $\mu \in[0, \lambda$ ) the $\mathrm{K}_{\mu}$-function of $\mathrm{F}$ is also absolutely continuous (density $\mathrm{k}_{\mu}$ ), and the following inequality holds:

$(5.2 .12) \quad k_{\mu}(x) / c(x ; \mu) \geq k_{\lambda}(x) / c(x ; \lambda) \quad(x>0)$.

PROOF. First take $\mu \in(0, \lambda)$. As we saw in the proof of theorem 5.2.4, $\hat{F}(\tau+\mu) / \hat{F}(\tau+\lambda)$ is comp mon, so by Bernstein's theorem there exists a rightcontinuous, nondecreasing function $\mathrm{K}_{\lambda, \mu}$, zero for negative arguments, such that $\hat{F}(\tau+\mu) / \hat{F}(\tau+\lambda)=\hat{K}_{\lambda, \mu}(\tau)$. As $\varphi_{\lambda}=\hat{K}_{\lambda}$ and $\varphi_{\mu}=\hat{K}_{\mu}$ from (5.2.9) it follows that for all $x>0$

(5.2.13) $\int_{[0, x]} c(y ; \lambda) d K_{\mu}(y)=\int_{[0, x]} \int_{[0, x-y]} c(u ; \mu) d K_{\lambda}(u) d K_{\lambda, \mu}(y)$.

If $K_{\lambda}$ has a density $k_{\lambda}$, then, substituting $u+y=v$ in the right-hand side of (5.2.13) and then changing the order of integration, and noting that by lemma 5.1 .5

$$
K_{\lambda, \mu}(0)=\lim _{\tau \rightarrow \infty} \hat{F}(\tau+\mu) / \hat{F}(\tau+\lambda)=1
$$

we obtain the absolute continuity of $\mathrm{K}_{\mu}$, with density $\mathrm{k}_{\mu}$ given by

$(5.2 .14) \quad k_{\mu}(x)=c(x ; \lambda)^{-1}\left\{c(x ; \mu) k_{\lambda}(x)+\int_{(0, x]} c(x-y ; \mu) k_{\lambda}(x-y) d K_{\lambda, \mu}(y)\right\}$.

For the case $\mu=0$, we let $\mu \downarrow 0$ in $(5.2 .9)$ to obtain

$(5.2 .15) \quad c_{\lambda}\left\{\hat{K}_{0}(\tau)-\hat{K}_{0}(\tau+\lambda)\right\}=\frac{\ddot{F}(\tau)}{F(\tau+\lambda)} \frac{d}{d \tau}\left[-\hat{K}_{\lambda}(\tau)\right]$,

which by $(5.1 .13)$ is equivalent to

$$
\int_{[0, x]} c(y ; \lambda) d K_{0}(y)=\int_{[0, x]} y d K_{\lambda}(y)+\int_{[0, x]} K_{\lambda}(x-y)\left(1-e^{-\lambda y}\right) d K_{0}(y) .
$$

It follows that $K_{0}$ is absolutely continuous, with density $k_{0}$ given by

$(5.2 .17) k_{0}(x)=c(x ; \lambda)^{-1}\left\{x k_{\lambda}(x)+\int_{[0, x]} k_{\lambda}(x-y)\left(1-e^{-\lambda y}\right) d k_{0}(y)\right\}$.

Finally, $(5.2 .12)$ is obtained from $(5.2 .14)$ and $(5.2 .17)$. 
REMARK 5.2.6. In case $\mu=0$ in the preceding theorem we can reverse matters. Suppose that the $K_{0}$-function of $F \in F_{\lambda}$ has a density $k_{0}$, then from $(5.2 .16)$ it follows that the $\mathrm{K}_{\lambda}$-function of $\mathrm{F}$ satisfies

$$
\begin{aligned}
\int_{[0, x]} y d k_{\lambda}(y) & =\int_{[0, x]}\left\{c(y ; \lambda) k_{0}(y) \cdots\right. \\
& \left.-\int_{[0, y]}\left(1-e^{-\lambda(y-z)}\right) k_{0}(y-z) d k_{\lambda}(z)\right\} d y .
\end{aligned}
$$

Hence, as $\mathrm{K}_{\lambda}(0)=0, \mathrm{~K}_{\lambda}$ is absolutely continuous and has a density $\mathrm{k}_{\lambda}$, that satisfies $(5.2 .17)$.

Thus we have proved that if $F \in F_{\lambda}$, then the absolute continuity of the $K_{\lambda}-$ function and that of the $\mathrm{K}_{\mathrm{o}}$-function of $\mathrm{F}$ are equivalent. Furthermore, in view of theorem 5.2.4 it now follows more generally that if $F \in F_{\lambda}$ and if $0 \leq \mu<\lambda$, then the $\mathrm{K}_{\lambda}$-function of $\mathrm{F}$ is absolutely continuous iff the $\mathrm{K}_{\mu}-$ function of $\mathrm{F}$ is absolutely continuous.

The absolute continuity of the $\mathrm{K}_{\lambda}$-function of $F \in F_{\lambda}$ is also sufficient for the absolute continuity of $F(x)-F(0)$. This observation generalizes a theorem by Tucker (1962) or Fisz \& Varadarajan (1963) (our theorem 1.7.10), if restricted to the half-line. In our case the proof is very simple.

THEOREM 5.2.7. If $0 \leq \lambda \leq \infty$ and if $F \in F_{\lambda}$ (with $F(0)>0$ if $\lambda=\infty$ ) has an absolutely continuous $k_{\lambda}$-function, then $F(x)-F(0)$ is absolutely continuous.

PROOF. If $k_{\lambda}$ has a density $k_{\lambda}$, then the right-hand side of (5.1.7) or (5.1.11) is absolutely continuous with density u given by

$$
u(x)=\int_{[0, x]} k_{\lambda}(x-y) d F(y) \quad(x>0) .
$$

As $c(x ; \lambda)>0$ for all $x>0$, from $(5.1 .7)$ or $(5.1 .11)$ it now follows that

$$
F(x)-F(0)=\int_{[0, x]} d F(y)=\int_{(0, x]} c(y ; \lambda)^{-1} u(y) d y,
$$

and hence $F(x)-F(0)$ is absolutely continuous with density $f_{0}$ given by

$$
(5.2 .19) \quad f_{0}(x)=c(x ; \lambda)^{-1} \int_{[0, x]} k_{\lambda}(x-y) d F(y) \quad(x>0) .
$$


COROLLARY 5.2.8. Let $0 \leq \lambda<\infty$ and let $F \in F_{\lambda}$. If the $k_{\lambda}$-function of $F$ is absolutely continuous with

$(5.2 .20) \int_{(0, \infty)} c(x ; \lambda)^{-1} d K_{\lambda}(x)=\infty$,

then $F$ is absolutely continuous, i.e. $F \in F_{\lambda}^{\prime}$.

PROOF. Combine theorem 5.2.7 and lemma 5.2.2.

REMARK 5.2.9. The $\mathrm{K}_{\mathrm{O}}$-function of a df $\mathrm{F} \in \mathrm{F}_{\mathrm{O}}^{\prime}$ satisfies $(5.2 .20)$ with $\lambda=0$, but it is not necessarily absolutely continuous: if $k_{0}$ has an absolutely continuous part, i.e. $k_{0}=K_{0}^{(1)}+K_{0}^{(2)}$ with $K_{0}^{(i)}$ nondecreasing $(i=1,2)$ and $\mathrm{K}_{0}^{(1)} \not 0$ absolutely continuous, then fxom (1.6.3) it is seen that $\mathrm{F}$ has an absolutely continuous component, and hence is itself absolutely continuous. By remark 5.2.6 it follows that also the $k_{\lambda}$-function of a df $F \in F_{\lambda}^{\prime \prime}$ may not be expected to be always absolutely continuous.

\subsection{Further properties of the $F_{\lambda}$ 's, examples}

When considering properties and examples of df's in $F_{\lambda}$, we shall frequently use the characterization of $F_{\lambda}$ given by lemma 5.1.4. The $\varphi_{\lambda}$-function of a df that depends on some parameter $\nu$, will then be denoted by $\varphi_{\lambda}(\nu)$, and we shall use the same notation without further comment in several different situations.

We start with some properties of the classes $F_{\lambda}$. The first of them is well known for $F_{0}$ (cf. theorem 1.4.3).

THEOREM 5.3.1. For $0 \leq \lambda \leq \infty$ the class $F_{\lambda}$ is closed under weak convergence, i.e. a df $F$ on $[0, \infty)$, for which there exist $F_{n} \in F_{\lambda}(n \in \mathbb{N})$ such that $\hat{\mathrm{F}}(\tau)=\lim _{\mathrm{n} \rightarrow \infty} \hat{\mathrm{F}}_{\mathrm{n}}(\tau)(\tau \geq 0)$, is again in $F_{\lambda}$.

PROOF. By the definition of $F_{\infty}$ it is sufficient to consider the case

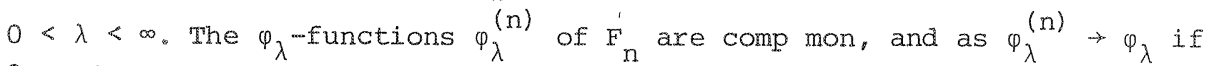
$\hat{F}_{n} \rightarrow \hat{F}(n \rightarrow \infty)$, it follows that $\varphi_{\lambda}$ is comp mon too. So $F \in F_{\lambda}$.

It turns out that every $F_{\lambda}(0 \leq \lambda \leq \infty)$ is closed under translations, but only $F_{0}$ and $F_{\infty}$ are closed under scale transformations. 
THEOREM 5.3.2. If $0 \leq \lambda \leq \infty$ and $a>0$, then

(i) A df $F$ on $[0, \infty)$ is in $F_{\lambda}$ iff the df $F_{a}(x):=F(x-a)$ is in $F_{\lambda}$;

(ii) $A$ df $F$ on $[0, \infty)$ is in $F_{\lambda}$ iff the $d f F_{a}(x):=F(a x)$ is in $F_{a \lambda}{ }^{\circ}$

PROOF. The theorem is known for $\lambda=0$ and follows for $\lambda=\infty$ as soon as it has been proved for finite $\lambda$ 's. So let $0<\lambda<\infty$ and $a>0$. In case (i) we have

$$
\hat{\mathrm{F}}_{\mathrm{a}}(\tau)=\mathrm{e}^{-\mathrm{a} \tau_{\hat{F}}}(\tau)
$$

by which one easily obtains the following relation between the $\varphi_{\lambda}$-functions of $F_{a}$ and $F$ :

(5.3.1) $\varphi_{\lambda}^{(a)}(\tau)=c(a ; \lambda)+e^{-a \lambda} \varphi_{\lambda}(\tau)$.

As $\lim _{\tau \rightarrow \infty} \varphi_{\lambda}(\tau)=c(\ell(F) ; \lambda) \geq 0$ (cf. lemma 5.1.6), it follows that $\varphi_{\lambda}$ is comp mon iff $\varphi_{\lambda}^{(a)}$ is comp mon. Hence (i) is proved. In case (ii) $\hat{F}$ and $\hat{F}$ a are related by

$$
\hat{\mathrm{F}}_{\mathrm{a}}(\tau)=\hat{\mathrm{F}}(\tau / \mathrm{a})
$$

from which it is easily seen that

$$
(5.3 .2) \quad \varphi_{a \lambda}^{(a)}(\tau)=\left\{c_{a \lambda} / c_{\lambda}\right\} \varphi_{\lambda}(\tau / a)
$$

It follows that $\varphi_{\lambda}$ is comp mon iff $\varphi_{a \lambda}^{(a)}$ is comp mon, and (ii) is proved.

In view of part (ii) of the preceding theorem, for many purposes, such as asymptotic behaviour and properties of moments, it is sufficient to consider, apart from $F_{0}$ and $F_{\infty}$, only, say, the class $F_{1}$ in stead of all classes $F_{\lambda}$ for $0<\lambda<\infty$. Still, the monotonicity of $F_{\lambda}$ is an interesting property, and an explicit definition of the $F_{\lambda}^{\prime}$ 's is needed to define the class $F_{\infty}$. Furthermore, we note that, if we want to consider specifically lattice distributions, i.e. distributions on the fixed lattice $\mathbb{N}_{0}$, then the transformation $F_{a}(x)=F(a x)$ is not possible (unless $\left.a^{-1} \in \mathbb{N}\right)$ : the classes $C_{\alpha}(0 \leq \alpha \leq 1)$ are essentially distinct.

In the following theorem we state some properties of the $F_{\lambda}{ }^{\prime} s$ for $0 \leq \lambda \leq \infty$, which are well known or trivial for $\lambda=0$. 
THEOREM 5.3.3. FOr $0 \leq \lambda \leq \infty$ the following properties hold:

(i) If $F \in F_{\lambda}$ and $0 \leq \nu<\infty$, then $\hat{F}(\tau+\nu) / \hat{F}(\nu) \in F_{\lambda}$.

(ii) If $F \in F_{\lambda}$ and $0 \leq \nu \leq 1$, then $\hat{F}^{\nu} \in F_{\lambda}$.

(iii) If $F \in F_{\lambda}$ and $0 \leq \nu<\infty$, then $\hat{\mathrm{F}}(\nu) \hat{\mathrm{F}}(\tau) / \hat{\mathrm{F}}(\tau+\nu) \in F_{\lambda}$.

(iv) If $F \in F_{\lambda}, n \in \mathbb{N}$ and $0 \leq \nu \leq \lambda / n$, then $\underset{k=0}{n-1} \hat{\mathrm{F}}(\tau+k \nu) / \hat{\mathrm{F}}(\mathrm{k} \nu) \in F_{\nu}$.

PROOF. It is sufficient to consider the case $0<\lambda<\infty$. The proof is then analogous to that of theorem 2.4 .2 in the discrete case (use lemma 5.1.4). $\square$

The case $\lambda=\infty$ in (iv) can be used to construct examples of df's in $F_{\lambda}$; we state it as a corollary.

COROLLARY 5.3.4. If $\mathrm{F} \in \mathrm{F}_{\infty}$ and if $0 \leq \lambda<\infty$, then for all $\mathrm{n} \in \mathbb{N}$

$(5.3 .3) \prod_{k=0}^{n-1} \hat{F}(\tau+k \lambda) / \hat{F}(k \lambda) \in F_{\lambda}$

We note that if $F(0)=0$ then in $(5.3 .3)$ we cannot take $n=\infty$. In fact, the infinite product converges iff the series

$$
\sum_{k=0}^{\infty}\{1-\hat{F}(\tau+k \lambda) / \hat{F}(k \lambda)\}=c_{\tau}^{-1} \sum_{k=0}^{\infty} \hat{k}_{\tau}(k \lambda)
$$

is convergent, but in view of lemma 5.2 .2 and $(5.2 .6)$ this can only be the case if $F(0)>0$. If $F \in F_{\infty}^{+}$, however, then from theorem 5.2 .3 it is seen that the infinite product yields a PLST in $F_{\lambda}$ indeed. This also follows from the following characterization of $F_{\lambda}$ in terms of $F_{\infty}^{+}$(cf. theorem 2.4.5).

THEOREM 5.3.5. Let $\mathrm{F}$ be a df on $[0, \infty)$, let $0<\lambda<\infty$ and define the function $\psi_{\lambda}$ by

(5.3.4) $\quad \psi_{\lambda}(\tau):=\hat{F}(\lambda) \hat{F}(\tau) / \hat{F}(\tau+\lambda) \quad(\tau \geq 0)$.

Then $F \in F_{\lambda}$ iff $\psi_{\lambda}$ is a PLST in $F_{\infty}^{+}$.

PROOF. If $F \in F_{\lambda}$, then according to theorem 5.1.9 $\psi_{\lambda}$ is a PLST $\hat{F}_{\lambda}$, say, with $F_{\lambda}(0)=\hat{F}(\lambda) \exp [\lambda \ell(F)]>0$. In this case the $\varphi_{\infty}$-function of $F_{\lambda}$ is given by $(5.3 .5) \varphi_{\infty}^{(\lambda)}(\tau)=1-e^{\lambda \ell(F)}+e^{\lambda \ell(F)} C_{\lambda}^{-1} \varphi_{\lambda}(\tau)$, 
and as $\varphi_{\lambda}$ and $\varphi_{\infty}^{(\lambda)}$ are both nonnegative functions, it follows that $\varphi_{\lambda}$ is comp mon iff $\varphi_{\infty}^{(\lambda)}$ is. Applying lemma 5.1.4 now proves the theorem.

COROLLARY 5.3.6. A df $F$ on $[0, \infty)$ is in $F_{\infty}^{+}$iff $\prod_{k=0}^{\infty} \hat{F}(\tau+k \lambda) / \hat{F}(k \lambda)$ is a PLST $\operatorname{in} F_{\lambda}$.

Using theorems 5.2.4 and 5.3.5 we can improve part (iii) of theorem 5.3.3 in the following way.

COROLIARY 5.3.7. If $0 \leq \lambda \leq \infty$ and if $F \in F_{\lambda}$, then for all $\nu \in[0, \infty)$ the function $\hat{F^{\prime}}(\nu) \hat{F}(\tau) / \hat{F}(\tau+\nu)$ is a PIST $\hat{F}_{\nu^{\prime}}$ say, while $F_{\nu} \in \hat{F}_{\lambda}^{+}$if $\nu>\lambda$, and $F_{\nu} \in F_{\infty}^{+}$if $0 \leq \nu \leq \lambda$.

As a last property of the $F_{\lambda}^{\prime}$ 's we prove that $\underset{\lambda>0}{U} F_{\lambda}$ is dense in $F_{0}$ in the sense of weak convergence.

THEOREM 5.3.8. If $F \in F_{0}$, then there exists a decreasing sequence $\left\{\lambda_{n}\right\}$ with $\lambda_{n} \rightarrow 0$ and there are $F_{n} \in F_{\lambda_{n}}(n \in \mathbb{N})$ such that

$$
\hat{\mathrm{F}}(\tau)=\lim _{n \rightarrow \infty} \hat{\mathrm{F}}_{n}(\tau) \quad(\tau \geq 0) \text {. }
$$

PROOF. As $\mathrm{F}_{0}^{+}$coincides with the set of compound Poisson distributions on $[0, \infty)$ (cf. theorem 1.6 .6$)$, for $F \in F_{0}^{+}$we can give a proof along the same lines as in theorem 2.4 .4 for the discrete case. For an arbitrary $F \in F_{0}$ this proof and the proof of De Finetti's theorem (theorem 1.4.15) suggest the following choice: take $\lambda_{n}=n^{-2}$, and $F_{n}$ such that for $n \geq 2$

$$
\text { (5.3.6) } \quad \hat{F}_{n}(\tau)=\prod_{k=0}^{n-1} \frac{1-n^{-\frac{1}{2}} \hat{G}_{n}\left(k / n^{2}\right)}{1-n^{-\frac{1}{2}} \hat{G}_{n}\left(\tau+k / n^{2}\right)} \quad(\tau \geq 0),
$$

where $G_{n}$ is defined by

$$
\hat{G}_{n}(\tau):=\hat{F}(\tau)^{1 / n^{\frac{1}{2}}} \quad(\tau \geq 0),
$$

which because of the inf div of $F$ is indeed a PLST. Using in corollary 5.3 .4 a compound-geometric- $\left(n^{-\frac{1}{2}}, G_{n}\right)$ distribution, we see that $F_{n} \in F_{\lambda_{n}}$. Next we rewrite $\hat{\mathrm{F}}_{\mathrm{n}}$ as follows: 
$(5.3 .7) \quad \hat{F}_{n}(\tau)=\left\{1-n^{-\frac{1}{2}}\left(1-\hat{G}_{n}(\tau)\right)\right\}^{n} \prod_{k=0}^{n-1}\left\{1+g_{k, n}(\tau)\right\}$

where

$g_{k, n}(\tau):=\frac{\hat{G}_{n}\left(\tau+k / n^{2}\right)-\bar{G}_{n}\left(k / n^{2}\right)+1-\hat{G}_{n}(\tau)}{n^{\frac{1}{2}}+\tilde{G}_{n}(\tau)-1} \quad(n \in \mathbb{N} ; k=0,1, \ldots, n-1 ; \tau \geq 0)$.

Observing that for all $\tau \geq 0$ and all $k \in\{0,1, \ldots, n-1\}$ we have

$$
\begin{aligned}
& \hat{G}_{n}(\tau)-\hat{G}_{n}\left(\tau+k / n^{2}\right) \leq \hat{G}_{n}(\tau)-\hat{G}_{n}(\tau+1 / n)= \\
& =\int_{[0, \infty)} e^{-\tau x}\left\{1-e^{-x / n}\right\} d G_{n}(x) \leq 1-\hat{G}_{n}(1 / n),
\end{aligned}
$$

for $\left|g_{k, n}(\tau)\right|$ we obtain an upperbound, independent of $k$ and $\tau$ :

(5.3.8) $\left|g_{k, n}(\tau)\right| \leq 2\left(n^{\frac{1}{2}}-1\right)^{-1}\left\{1-\hat{G}_{n}(1 / n)\right\} \quad(n \in \mathbb{N} ; k=0,1, \ldots, n-1 ; \tau \geq 0)$.

Now define $\delta_{n}:=1-\hat{F}(1 / n)$, then $\delta_{n} \in(0,1)$ and

$$
\begin{aligned}
& n^{\frac{1}{2}}\left\{1-\hat{G}_{n}(1 / n)\right\}=n^{\frac{3}{2}}\left\{1-\left(1-\delta_{n}\right)^{1 / n^{\frac{1}{2}}}\right\}=-n^{\frac{1}{2}} \sum_{k=1}^{\infty}\left(\begin{array}{c}
1 / n^{\frac{1}{2}} \\
k
\end{array}\right)\left(-\delta_{n}\right)^{k}= \\
& =\delta_{n} \sum_{k=1}^{\infty} \frac{1}{k}\left(\begin{array}{c}
1 / n^{\frac{1}{2}}-1 \\
k-1
\end{array}\right)\left(-\delta_{n}\right)^{k-1} \leq \delta_{n} \sum_{l=0}^{\infty}\left(\begin{array}{c}
1 / n^{\frac{1}{2}}-1 \\
l
\end{array}\right)\left(-\delta_{n}\right)^{l}=\delta_{n}\left(1-\delta_{n}\right)^{1 / n^{\frac{1}{2}}-1},
\end{aligned}
$$

which, as $\delta_{n}=0(1)(n \rightarrow \infty)$, tends to zero as $n \rightarrow \infty$. It follows that

$$
1-\hat{G}_{n}(1 / n)=o\left(n^{-\frac{1}{2}}\right) \quad(n \rightarrow \infty),
$$

and hence by $(5.3 .8)$

(5.3.9) $\quad \forall_{k \in\{0,1, \ldots, n-1\}} \forall_{\tau \geq 0}\left|g_{k, n}(\tau)\right| \leq \varepsilon_{n}$, with $\varepsilon_{n}=o\left(\frac{1}{n}\right) \quad(n \rightarrow \infty)$.

We can write now the following inequalities:

$$
\left(1-\varepsilon_{n}\right)^{n} \leq \prod_{k=0}^{n-1}\left\{1+g_{k, n}(\tau)\right\} \leq\left(1+\varepsilon_{n}\right)^{n}
$$

from which by the asymptotic behaviour of $\varepsilon_{n}$ it is easily seen that

$$
\text { (5.3.10) } \lim _{n \rightarrow \infty} \prod_{k=0}^{n-1}\left\{1+g_{k, n}(\tau)\right\}=1 \quad(\tau \geq 0)
$$


Turning to the first factor in the right-hand side of (5.3.7) we write

$$
\left\{1-n^{-\frac{1}{2} / 2}\left(1-\hat{G}_{n}(\tau)\right)\right\}^{n}=\exp \left[n \log \left(1-c_{n}\right)\right] .
$$

with $c_{n}:=n^{-\frac{1}{2}}\left(1-\hat{G}_{n}(\tau)\right)$. Using the fact that

$$
-x-x^{2} \leq \log (1-x) \leq-x \text { for } 0 \leq x \leq \frac{1}{2},
$$

and observing that $\mathrm{nc}_{\mathrm{n}} \rightarrow-\log \hat{\mathrm{F}}(\tau)$ and $\mathrm{nc}_{\mathrm{n}}^{2} \rightarrow 0$ as $\mathrm{n} \rightarrow \infty$, we see that

(5.3.11) $\lim _{n \rightarrow \infty}\left\{1-n^{-\frac{1}{2}}\left(1-\hat{G}_{n}(\tau)\right)\right\}^{n}=\hat{F}(\tau) \quad(\tau \geq 0)$.

Combining this result with $(5.3 .10)$, from $(5.3 .7)$ we conclude that $\lim _{n \rightarrow \infty} \hat{F}_{n}(\tau)=\hat{F}(\tau)$.

To construct examples of df's in $F_{\lambda}$ we need, apart from lemma 1.3.8, the following lemma's on comp mon functions. They can easily be proved by use of Bernstein's theorem and partial fraction expansions.

LEMMA 5.3.9. For $\mu_{3}>0$ the function $\varphi$, defined by

$$
\varphi(\tau):=\frac{\mu_{1}+\mu_{2} \tau}{\mu_{3}+\tau} \quad(\tau>0),
$$

is comp mon iff $\mu_{2} \geq 0$ and $\mu_{1} \geq \mu_{2} \mu_{3}$.

LEMMA 5.3.10. For $\mu_{3}>0$ and $\mu_{4}>0$ the function $\varphi$, defined by

$$
\varphi(\tau):=\frac{\mu_{1}+\mu_{2} \tau}{\left(\mu_{3}+\tau\right)\left(\mu_{4}+\tau\right)} \quad(\tau>0),
$$

is comp mon iff $\mu_{2} \geq 0$ and $\mu_{1} \geq \mu_{2} \min \left(\mu_{3}, \mu_{4}\right)$.

LEMMA 5.3.11. For $\mu_{3}>0$ and $\mu_{4}>0$ the function $\varphi$, defined by

$$
\varphi(\tau):=\frac{\left(\mu_{1}+\tau\right)\left(\mu_{2}+\tau\right)}{\left(\mu_{3}+\tau\right)\left(\mu_{4}+\tau\right)} \quad(\tau>0),
$$

is comp mon iff $\min \left(\mu_{1}, \mu_{2}\right) \geq \min \left(\mu_{3}, \mu_{4}\right), \mu_{1} \mu_{2} \geq \mu_{3} \mu_{4}$ and $\mu_{1}+\mu_{2} \geq \mu_{3}+\mu_{4}$.

Now we mention some simple examples of distributions in $F_{\lambda}$. In the next section some more examples for $F_{\infty}$ will be given. 
1. Consider the exponential distribution with parameter $\mu>0$; its pdf and PLST is given by

$$
f(x)=\mu e^{-\mu x} \quad(x>0) \text {, and } \hat{F}(\tau)=\frac{\mu}{\mu+\tau} .
$$

Calculating the $\varphi_{\lambda}$-function of $F$, we obtain

$(5.3 .12) \frac{\mu}{\mu+\tau} \in F_{\infty}^{\prime} \quad(\mu>0)$.

In fact, the $\mathrm{K}_{\lambda}$-function of $\mathrm{F}$ has a density $\mathrm{k}_{\lambda}$ given by

$$
k_{\lambda}(x)=\lambda c_{\lambda} e^{-(\lambda+\mu) x} \quad(x>0 ; 0 \leq \lambda<\infty) .
$$

2. The gamma distribution with parameters $\mu>0$ and $\nu>0$ has pdf and PLST given by

$$
f(x)=\frac{\mu^{\nu}}{\Gamma(\nu)} x^{\nu-1} e^{-\mu x}(x>0), \text { and } \hat{F}(\tau)=\left\{\frac{\mu}{\mu+\tau}\right\}^{\nu}
$$

In view of theorem 5.3.3(ii), from example 1. we conclude that

$$
\text { (5.3.13) }\left\{\frac{\mu}{\mu+\tau}\right\}^{\nu} \in F_{\infty}^{\prime} \quad(\mu>0 ; 0<\nu \leq 1) .
$$

but, as for the discrete analogue of the gamma distribution (cf. example 2, p. 56), we have

$$
(5.3 .14) \quad\left\{\frac{\mu}{\mu+\tau}\right\}^{\nu} \& \underset{\lambda>0}{U} F_{\lambda} \quad(\mu>0 ; \nu>1) .
$$

To show this we put $\nu=1+\varepsilon$ with $\varepsilon>0$, and we calculate the $\varphi_{\lambda}$-function of $\mathrm{F}$ :

$$
\varphi_{\lambda}(\tau)=c_{\lambda}\left\{1-\left(\frac{\mu+\tau}{\mu+\lambda+\tau}\right)^{1+\varepsilon}\right\}=-\sum_{k=1}^{\infty}\left(\begin{array}{c}
1+\varepsilon \\
k
\end{array}\right)(-\lambda)^{k}(\mu+\lambda+\tau)^{-k}
$$

which can be written as the LT of a function $k_{\lambda}$ that satisfies

$\lambda^{-1} e^{(\lambda+\mu) x_{k}}(x)=\sum_{k=1}^{\infty}\left(\begin{array}{c}1+\varepsilon \\ k\end{array}\right) \frac{(-\lambda x)^{k-1}}{(k-1) !}=1+\varepsilon-\varepsilon(1+\varepsilon) \sum_{k=1}^{\infty}\left(\begin{array}{c}k-1-\varepsilon \\ k-1\end{array}\right) \frac{(\lambda x)^{k}}{k(k+1) !}$.

As for $0<\varepsilon \leq 1$ this is less than $(1+\varepsilon)\{1-\varepsilon \lambda x / 2\}$, which tends to $-\infty$ as $x \rightarrow \infty,(5.3 .14)$ is proved for $1<v \leq 2$. Finally, from this and theorem 5.3 .3 (ii) it is easily seen that $(5.3 .14)$ also holds for $v>2$. 
3. In view of example 1 we can take $\hat{F}(\tau)=\mu /(\mu+\tau)$ in corollary 5.3 .4 to obtain

(5.3.15) $\prod_{k=0}^{n-1} \frac{\mu+k \lambda}{\mu+k \lambda+\tau} \in F_{\lambda}^{8} \quad(\mu>0 ; n \in \mathbb{N} ; 0 \leq \lambda<\infty)$.

4. For a product of two exponential PLST's, which, obviously, can be given the form (5.3.15) with $\mathrm{n}=2$, we have

(5.3.16) $\frac{\mu}{\mu+\tau} \frac{\mu+\nu}{\mu+\nu+\tau} \in F_{\lambda}^{8} \Leftrightarrow \lambda \leq \nu \quad(\mu>0 ; \nu \geq 0)$.

In fact, the $\varphi_{\lambda}$-function can easily be seen to be the LT of the function $k_{\lambda}$ given by

$$
k_{\lambda}(x)=\lambda c_{\lambda} \nu^{-1}\left\{(\nu-\lambda)+(\nu+\lambda) e^{-\nu x}\right\} e^{-(\mu+\lambda) x} \quad(x>0)
$$

from which it follows that $\varphi_{\lambda}$ is comp mon, or, equivalently, $k_{\lambda}(x) \geq 0$ for all $\mathrm{x}>0$, iff $\lambda \leq \nu$.

5. A quotient of two exponential PLST's with parameters $\mu>0$ and $\nu>0$, respectively, is again a PLST iff $\mu \leq \nu$ (cf. lemma 5.3.9). We then have (5.3.17) $\frac{\mu}{\mu+\tau} / \frac{\nu}{\nu+\tau} \in F_{\infty}^{+} \quad(0<\mu \leq \nu)$. In fact, the $\mathrm{K}_{\infty}$-function has a density $k_{\infty}$ given by

$$
k_{\infty}(x)=(\nu-\mu) e^{-v x} \quad(x>0) .
$$

6. Taking $F$ in corollaries 5.3 .4 and 5.3 .6 compound geometric, we get

(5.3.18) $\prod_{k=0}^{n-1} \frac{1-p \hat{G}(k \lambda)}{1-p \hat{G}(\tau+k \lambda)} \in F_{\lambda} \quad(n \in \mathbb{N} \cup\{\infty\} ; 0 \leq p<1 ; G$ is df on $[0, \infty))$.

7. Choose in $(5.3 .18) \hat{G}(\tau)=\mu /(\mu+\tau)$, then it follows that

(5.3.19) $\prod_{k=0}^{n-1} \frac{\mu_{1}+k \lambda}{\mu_{1}+k \lambda+\tau} / \frac{\mu_{2}+k \lambda}{\mu_{2}+k \lambda+\tau} \in F_{\lambda} \quad\left(n \in \mathbb{N} \cup\{\infty\} ; 0<\mu_{1} \leq \mu_{2}\right)$.

Note that for $\mathrm{n}=1$ we get example 5 .

8. Finally, we want to show that for positive $\mu_{1}, \mu_{2}$ and $\mu_{3}$ 
$(5.3 .20) \frac{\mu_{1}}{\mu_{1}+\tau} \frac{\mu_{3}}{\mu_{3}+\tau} /\left\{\frac{\mu_{2}}{\mu_{2}+\tau}\right\}^{2} \in F_{\infty}^{+} \Leftrightarrow \frac{s_{2}}{2}\left(\mu_{1}+\mu_{3}\right) \leq \mu_{2} \leq \max \left(\mu_{1}, \mu_{3}\right)$.

First we note that according to lemma 5.3.11 for the function in (5.3.20) to be a PLST it is necessary and sufficient that $\mu_{2} \geq \frac{1}{2}\left(\mu_{1}+\mu_{3}\right)$. Calculating the $\varphi_{\infty}$-function in that case, we find

$$
\varphi_{\infty}(\tau)=\left(\mu_{2}+\tau\right)^{-2}\left\{\left(\mu_{2}^{2}-\mu_{1} \mu_{3}\right)+\left(2 \mu_{2}-\mu_{1}-\mu_{3}\right) \tau\right\},
$$

from which by lemma 5.3 .10 it follows that $\varphi_{\infty}$ is comp mon iff $\mu_{2}^{2}-\mu_{1} \mu_{3} \geq$ $\geq\left(2 \mu_{2}-\mu_{1}-\mu_{3}\right) \mu_{2}$, i.e. $\left(\mu_{3}-\mu_{2}\right)\left(\mu_{2}-\mu_{1}\right) \geq 0$, or $\mu_{2} \leq \max \left(\mu_{1}, \mu_{3}\right)$.

\subsection{The class $F_{\infty}$}

In this section we study the class $F_{\infty}:=\lim _{\lambda \rightarrow \infty} F_{\lambda}$ in more detajl, and we do so mainly for the following two reasons. In the first place, $F_{\infty}$ seems to be the analogue of $C_{0}$ for distributions on $[0, \infty)$. In fact, similarly to the way $F_{\infty}$ is defined, $C_{0}$ can be obtained as the limit of the discrete analogues of the $F_{\lambda}$ 's, the classes $C_{\alpha}$, for $\alpha \downarrow 0$. Furthermore, it will turn out that $F_{\infty}$ has properties, very similar to those of $C_{O}$, and $F_{\infty}$ contains the compound geometric distributions on $[0, \infty)$ just as $F_{0}$ contains the compound poisson ones. Secondly we study $F_{\infty}$ because of its interesting relations with other classes of functions occurring in probability theory, such as the standard p-functions, the renewal densities and the potential kernels. These relations will be discussed briefly in the next section.

Our first aim is to look for some basic properties of $F_{\infty}$ as available for $F_{0}$ (cf. theorems 1.6 .1 and 1.6.2). The relation between $C_{0}$ and $R_{0}$ (cf. section 4.1 ) suggests the existence of a relation between $F_{\infty}$ and the class of continuous analogues of the renewal sequences, the (standard) p-functions (cf. section 5). By a method used by Kingman (1972) for p-functions, we can derive a canonical representation for the PLST's in $F_{\infty}$. The starting point for this is the observation that $F_{\infty}^{+}$i's dense in $\left\{F \in F_{\infty} \mid l(F)=0\right\}$ in the sense of weak convergence (this can be considered as an analogue of De Finetti's theorem (theorem 1.4.15) for $F_{\infty}$ ): if $F \in F_{\infty}$ with $\ell(F)=0$, then by (5.1.13) and lemma 5.1.5 we can write

(5.4.1) $\hat{\mathrm{F}}(\tau)=\lim _{\lambda \rightarrow \infty} \frac{\hat{\mathrm{F}}(\tau+\lambda)}{1-c_{\lambda}^{-1} \hat{\mathrm{K}}_{\lambda}(\tau)}=\lim _{\lambda \rightarrow \infty} \frac{\hat{\mathrm{F}}(\lambda)}{1-c_{\lambda}^{-1} \hat{K}_{\lambda}(\tau)}=\lim _{n \rightarrow \infty} \frac{1-p_{n}}{1-p_{n} G_{n}(\tau)}$, 
where for $n \in \mathbb{N} \quad p_{n}:=1-\hat{F}(n) \in(0,1)$ and $\hat{G}_{n}(\tau):=c_{n}^{-1} \hat{K}_{n}(\tau) /\{1-\hat{F}(n)\}$ is a PLST (cf. $(5 \cdot 1.20))$. Using a different approach, however, we can derive the canonical representation much easiex. We shall do so now, and startwith a characterization of $F_{\infty}$ by means of a comp mon function. To this end we give the following definition.

DEFINITION 5.4.1. The $\psi_{\infty}$-function of a df $\mathrm{F}$ on $[0, \infty)$ is defined by

$$
\psi_{\infty}(\tau):=\frac{d}{d \tau} \hat{F}(\tau)^{-1}=-\hat{F}^{\theta}(\tau) / \hat{F}(\tau)^{2} \quad(\tau>0)
$$

We note the following relations with the $\varphi_{0}$-function and, if $F(0)>0$, with the $\varphi_{\infty}$-function of $F$ (cf. definition 5.1.3):

(5.4.2) $\psi_{\infty}(\tau)=\varphi_{0}(\tau) / \hat{F}(\tau)$ and $\psi_{\infty}(\tau)=-\varphi_{\infty}^{*}(\tau) / F(0)$.

As $\varphi_{\infty}(\tau) \geq 0(\tau>0)$, the latter relation shows that (cf. lemma 5.1.4(ii)) a df $F$ with $F(0)>0$ is in $F_{\infty}^{+}$iff the $\psi_{\infty}$-function of $F$ is comp mon. This characterization of $F_{\infty}^{+}$can be extended to $F_{\infty}$ in the following way (cf. lem$\operatorname{ma} 2.5 .6)$.

THEOREM 5.4.2. If $F$ is a df on $[0, \infty)$, then $F \in F_{\infty}$ with $\ell(F)=0$ iff the $\psi_{\infty}{ }^{-}$ function of $\mathrm{F}$ is comp mon.

PROOF. Let $F \in F_{\infty}$ with $\ell(F)=0$. Then by lemma $5.1 .4(i)$ for all $\lambda<\infty$ the $\varphi_{\lambda}$-function of $F$ is comp mon, and is related to the $\psi_{\infty}$-function of $F$ as follows:

$(5.4 .3)-\frac{d}{d \tau} \varphi_{\lambda}(\tau)=c_{\lambda} \hat{F}(\tau+\lambda)\left\{\psi_{\infty}(\tau)-\varphi_{0}(\tau+\lambda) / \hat{F}(\tau)\right\}$.

Division by $\hat{F}(\lambda)$, and use of the fact that $c_{\lambda} \rightarrow 1, \hat{F}(\tau+\lambda) / \hat{F}(\lambda) \rightarrow 1$ (cf. lema 5.1.5), and $\varphi_{0}(\tau+\lambda) \rightarrow 0$ (cf. lemma 5.1.6) as $\lambda \rightarrow \infty$, show that

$(5.4 .4) \quad \psi_{\infty}(\tau)=\lim _{\lambda \rightarrow \infty}-\frac{d}{d \tau} \varphi_{\lambda}(\tau) / \hat{F}(\lambda)$.

It follows that $\psi_{\infty}$ is comp mon, since it is the limit of a sequence of comp mon functions.

Conversely, let the $\psi_{\infty}$-function of a df $F$ on $[0, \infty)$ be comp mon, and let $0<\lambda<\infty$. As $\hat{F}$ is nonincreasing, the function

(5.4.5) $\varphi_{\lambda}(\tau) / \hat{F}(\tau+\lambda)=c_{\lambda}\left\{\hat{F}(\tau+\lambda)^{-1}-\hat{F}(\tau)^{-1}\right\}$ 
is nonnegative. Furthermore, it satisfies

$(5.4 .6)-\frac{d}{d \tau}\left[\varphi_{\lambda}(\tau) / \hat{F}(\tau+\lambda)\right]=c_{\lambda}\left\{\psi_{\infty}(\tau)-\psi_{\infty}(\tau+\lambda)\right\}$,

from which it follows that $\varphi_{\lambda}(\tau) / \hat{F}(\tau+\lambda)$, and hence $\varphi_{\lambda}(\tau)$, is comp mon. Thus we have proved that $F \in F_{\infty}$. In order to show that $l(F)=0$, we note that $\lim \psi_{\infty}(\tau)$ exists in $[0, \infty)$. Hence by lemma 5.1 .6 and $(5.4 .2), \ell(F)$ can $\tau \rightarrow \infty$ be obtained as follows:

$$
\ell(F)=\lim _{\tau \rightarrow \infty} \varphi_{O}(\tau)=\lim _{\tau \rightarrow \infty} \psi_{\infty}(\tau) \hat{F}(\tau)=F(0) \lim _{\tau \rightarrow \infty} \psi_{\infty}(\tau)
$$

from which it is seen that $\ell(F)=0$ if $F(0)=0$. As trivially $\ell(F)=0$ if $F(0)>0$, the theorem is proved.

Using Bernstein's theorem, from theorem 5.4.2 we immediately obtain a characterization of $F_{\infty}$ by a functional equation (cf. lemma 2.5.8, where a similar result is given for $C_{0}\left(=H_{1}\right.$; cf. theorem 2.5.11)).

COROLIARY 5.4.3. If $F$ is a df on $[0, \infty)$, then $F \in F_{\infty}$ with $\ell(F)=0$ iff there exists a right-continuous, nondecreasing function $L$ such that

$$
\int_{[0, x]} y d F(y)=\int_{[0, x]} F^{* 2}(x-y) d L(y) \quad(x \geq 0) .
$$

Relation (5.4.6) gives rise to a characterization of $F_{\infty}$ in terms of the $\varphi_{\lambda}{ }^{-}$ function for a fixed $\lambda \in[0, \infty)$. It shows in a sense which part of $F_{\lambda}$ consists of $F_{\infty}$-distributions.

THEOREM 5.4.4. If $F$ is a df on $\left[0, \infty\right.$, then $F \in F_{\infty}$ with $\ell(F)=0$ iff for some, and then for all, $\lambda \in[0, \infty)$ the function $\varphi_{\lambda}(\tau) / \hat{F}(\tau+\lambda)$ is comp mon.

PROOF. First we note that if $F \in F_{\infty}$ with $\ell(F)=0$, then by theorem 5.4 .2 the $\psi_{\infty}$-function of $F$ is comp mon, and, hence (cf. the second part of the proof of theorem 5.4.2) $\varphi_{\lambda}(\tau) / \hat{F}(\tau+\lambda)$ is comp mon for all $\lambda \in[0, \infty)$. From (5.4.2) it now follows that for $\lambda=0$ the theorem reduces to theorem 5.4.2. Therefore we take a fixed $\lambda \in(0, \infty)$ and suppose $\varphi_{\lambda}(\tau) / \hat{F}(\tau+\lambda)$ to be comp mon. Then the limit

(5.4.8) $a_{\lambda}:=\lim _{\tau \rightarrow \infty} \varphi_{\lambda}(\tau) / \hat{F}(\tau+\lambda)=c_{\lambda} \lim _{\tau \rightarrow \infty} \frac{\hat{F}(\tau)-\hat{F}(\tau+\lambda)}{\hat{F}(\tau) \hat{F}(\tau+\lambda)}$ 
exists in $[0, \infty)$. For all $\tau>0$ there exists $\theta_{\tau} \in(0,1)$ such that

$$
\hat{F}(\tau)-\hat{F}(\tau+\lambda)=-\lambda \hat{F}^{*}\left(\tau+\theta_{\tau} \lambda\right)
$$

and hence, as $\hat{\mathrm{F}}^{\prime}$ is nondecreasing,

$$
-\lambda \hat{F}^{\gamma}(\tau+\lambda) \leq \hat{F}(\tau)-\hat{F}(\tau+\lambda) \leq-\lambda \hat{F}^{\prime}(\tau) .
$$

or for $\tau>\lambda$

$$
\hat{F^{\prime}}(\tau)-\hat{F}(\tau+\lambda) \leq-\lambda \hat{F}^{\prime}(\tau) \leq \hat{F}(\tau-\lambda)-\hat{F}(\tau) .
$$

Now, dividing by $\hat{\mathrm{F}}(\tau)^{2}$, and using $(5.4 .8)$ and lemma 5.1 .5 , we see that $\psi_{\infty}$ satisfies

(5.4.9) $\left\{\lambda c_{\lambda}\right\}^{-1} a_{\lambda} e^{-\lambda \ell(F)} \leq \liminf _{\tau \rightarrow \infty} \psi_{\infty}(\tau) \leq \limsup _{\tau \rightarrow \infty} \psi_{\infty}(\tau) \leq\left\{\lambda c_{\lambda}\right\}^{-1} a_{\lambda} e^{\lambda \ell(F)}$.

If $F$ would be such that $\ell(F)>0$ (and so $F(0)=0$ ), $\psi_{\infty}$ would satisfy (cf.

Lemma 5.1 .6$)$

$$
\lim _{\tau \rightarrow \infty} \psi_{\infty}(\tau)=\lim _{\tau \rightarrow \infty} \varphi_{0}(\tau) / \hat{F}(\tau)=\infty
$$

which contradicts $(5.4 .9)$. It follows that $\ell(F)=0$, and hence

$$
(5.4 .10) \lim _{\tau \rightarrow \infty} \psi_{\infty}(\tau)=\left\{\lambda c_{\lambda}\right\}^{-1} a_{\lambda}
$$

Now $\psi_{\infty}$ can be written as

$$
\psi_{\infty}(\tau)=\left\{\lambda c_{\lambda}\right\}^{-1} a_{\lambda}+\sum_{k=0}^{\infty}\left\{\psi_{\infty}(\tau+k \lambda)-\psi_{\infty}(\tau+k \lambda+\lambda)\right\},
$$

which by $(5.4 .6)$ implies the comp mon of $\psi_{\infty}$. Hence by theorem $5.4 .2: F \in F_{\infty} \cdot \square$

Next, from theorem 5.4.2 we derive a representation theorem for $F_{\infty}$ that can be considered as an analogue of theorem 2.4 .8 , where a relation between $C_{0}$ and $C_{1}$ is given.

THEOREM 5.4.5. A function $\varphi$ on $[0, \infty)$ is the PLST of a df $F \in F_{\infty}$ with $\ell(F)=0$ iff there exists a df $H \in F_{0}$ such that $\varphi$ has the form

(5.4.11) $\varphi(\tau)=\{1-\log \hat{\mathrm{H}}(\tau)\}^{-1} \quad(\tau \geq 0)$.

PROOF. Let $F \in F_{\infty}$ with $l(F)=0$. Then the $\psi_{\infty}$-function $\psi_{\infty}$ of $F$ is comp mon. If we define the positive function $\psi$ on $[0, \infty)$ by 
$(5.4 .12) \quad \psi(\tau):=\exp \left[1-\hat{F}(\tau)^{-1}\right] \quad(\tau \geq 0)$

then it is seen that $\psi(0)=1$ and that the function

$$
-\frac{d}{d \tau} \log \psi(\tau)=\frac{d}{d \tau} \hat{F}(\tau)^{-1}=\psi_{\infty}(\tau)
$$

is comp mon. Now by theorem 1.6 .1 it follows that there exists a df $H \in F_{0}$ such that $\psi=\hat{H}$, and hence by $(5.4 .12) \hat{\mathrm{F}}$ can be represented in the form (5.4.11).

Conversely, suppose that a function $\varphi$ on $[0, \infty)$ has the form $(5.4 .11)$ with $\mathrm{H} \in \mathrm{F}_{\mathrm{O}}$. Then the $\varphi_{\mathrm{O}}$-function of $\mathrm{H}$.

$$
\varphi_{0}^{(H)}(\tau)=-\frac{d}{d \tau} \log \hat{H}(\tau)
$$

is comp mon, and as $-\log \hat{H}(\tau)$ is nonnegative, by lemma 1.3.8(vi) it follows that $\varphi$ is comp mon. Also $\varphi(0)=1$, and hence by Bernstein's theorem $\varphi$ is the PLST of a df $F$ on $[0, \infty)$, for which the $\psi_{\infty}$-function

$$
\psi_{\infty}(\tau)=\frac{\mathrm{d}}{\mathrm{d} \tau} \hat{\mathrm{F}}(\tau)^{-1}=\varphi_{0}^{(\mathrm{H})}(\tau)
$$

is comp mon. In view of theorem 5.4 .2 we conclude that $F \in F_{\infty}$ with $\ell(F)=0 . \square$

COROLLARY 5.4.6. If $F \in F_{\infty}$ with $\ell(F)=0$, then

$(5.4 .13) \hat{H}(\tau)=\exp \left[1-\hat{F}(\tau)^{-1}\right] \quad(\tau \geq 0)$

is the PLST of a df $\mathrm{H}$ in $F_{0}$. The $\mathrm{K}_{\mathrm{O}}$-function $\mathrm{K}_{\mathrm{O}}^{(\mathrm{H})}$ of $\mathrm{H}$ has the $\psi_{\infty}$-function of $F$ as its LST, and is therefore related to the $K_{0}$-function $K_{0}$ of $F$ as follows:

$(5.4 .14) \quad F * K_{0}^{(H)}=K_{0}$.

By letting $\tau \rightarrow \infty$ in $(5.4 .13)$, it is seen that $F(0)>0$ iff $H(0)>0$. Hence from the preceding theorem we obtain the following relation between $F_{0}^{+}$and $F_{\infty}^{+}$, which can also be established by use of the representations (1.6.12) and (1.6.13) for $F_{0}^{+}$and $F_{\infty}^{+}$, respectively (cf. the proof of theorem 2.4.8).

COROLLARY 5.4.7. A function $\varphi$ on $[0, \infty)$ is the PLST of a df $F \in F_{\infty}^{+}$iff there exists a df $\mathrm{H} \in F_{0}^{+}$such that $(5.4 .11)$ holds. 
We want to make the representation for PLST's in $F_{\infty}$, given by theorem 5.4.5, more explicit. We do so in the following theorem by introducing canonical quantities in such a way that several properties of $F_{\infty}$ are easily expressible in terms of them, and such that there exists a clear resemblance to the canonical representation for the LT of a standard p-function (cf. section 5).

THEOREM 5.4.8. A function $\varphi$ on $[0, \infty)$ is the PLST of a df $F \in F_{\infty}$ with $\ell(F)=0$ iff there exist $\gamma \geq 0$ and a right-continuous, nondecreasing function $N$ on $(0, \infty)$ with $N(\infty)=0$ and satisfying

$(5.4 .15) \quad \int_{(0,1]} \mathrm{x} d \mathrm{~N}(\mathrm{x})<\infty$.

such that $\varphi$ has the form

$(5.4 .16) \quad \varphi(\tau)=\left\{1+\gamma \tau+\int_{(0, \infty)}\left(1-e^{-\tau x}\right) d N(x)\right\}^{-1} \quad(\tau \geq 0)$

the representation $(\gamma, N)$ for $\varphi=\hat{F}$ is unique.

PROOF. The theorem is an immediate consequence of the preceding theorem, and theorem 1.7.1, where a representation $(\gamma, N)$ is given for the chf of an inf div df with finite left extremj.ty; using this representation for the df $\mathrm{H} \in \mathrm{F}_{\mathrm{O}}$ in $(5.4 .11)$, we get $(5.4 .16$;

It is useful to state the following corollary, which will be clear from corollary 1.7 .2 .

COROLLARY 5.4.9. If $F \in F_{\infty}$ with $\ell(F)=0$, then between the representation $(\gamma, N)$ in $(5.4 .16)$ for $\hat{F}$ and the $K_{0}$-function $K_{O}^{(H)}$ of the $d f H$, defined by (5.4.13), the following relations hold:

$(5.4 .17) \quad \gamma=K_{0}^{(H)}(0)$ and $N(x)=-\int_{(x, \infty)} \frac{1}{y} d K_{0}^{(H)}(y) \quad(x>0)$,

and, conversely,

$(5.4 .18) K_{0}^{(H)}(x)=\gamma+\int_{(0, x]} y d N(y) \quad(x \geq 0)$. 
The representation $(\gamma, N)$, given in $(5.4 .16)$, for the PLST of a df $F \in F_{\infty}$ with $\ell(F)=0$ will be called the canonical representation of $F \in F_{\infty}$. It has several simple properties, which we need in the sequel. They are summarized in the following lemma.

LEMMA 5.4.10. Let $F \in F_{\infty}$ with $\ell(F)=0$ and canonical representation $(\gamma, N)$. Then

(i) $\mathrm{N}$ is integrable on every finite interval, or, equivalently,

$(5.4 .19)-\int_{(0, x]} N(y) d y<\infty \quad(x>0)$.

(ii) $N$ is integrable on $(0, \infty)$ iff $\mu:=\int_{[0, \infty)} x d F(x)$ is finite, in which
case $(5.4 .20) \mu=\gamma-\int_{(0, \infty)} N(x) d x=\gamma+\int_{(0, \infty)} x d N(x)$.

(iii) The LT of $N$ exists on $(0, \infty)$ and can be expressed in $\hat{F}$ as follows:

(5.4.21) $\int_{(0, \infty)} e^{-\tau X} \mathbb{N}(x) d x=\gamma+\tau^{-1}-\{\tau \hat{F}(\tau)\}^{-1} \quad(\tau>0)$.

(iv) $\gamma$ can be obtained from $\hat{\mathrm{F}}$ by

$(5.4 .22) \quad \gamma=\lim _{\tau \rightarrow \infty}\{\tau \hat{\mathrm{F}}(\tau)\}^{-1}$.

(v) The $K_{0}$-function of $F$ is related to $(\gamma, N)$ as follows:

$(5.4 .23) \quad K_{0}(x)=\gamma F(x)+\int_{(0, x]} F(x-y) y d N(y) \quad(x \geq 0)$.

(vi) $F \in F_{\infty}^{+}$iff $\gamma=0$ and $N$ is bounded, i.e. $-N(0+)<\infty$.

PROOF。

(i) For all $x>0$, using Fubini's theorem, we can write

$(5.4 .24)-\int_{(0, x]} N(y) d y=\int_{(0, x]} y d N(y)-x N(x)$,

which by $(5.4 .15)$ is finite.

(ii) By Fubini's theorem it follows that 
$(5.4 .25)-\int_{(0, \infty)} N(x) d x=\int_{(0, \infty)} x d N(x)$

where the integrals may be both infinite. Further, by (5.4.14) and (5.4.18) we can write

$$
\mu=\int_{(0, \infty)} x d F(x)=\lim _{\tau \downarrow 0}-\hat{F}^{\prime}(\tau) / \hat{F}(\tau)^{2}=\lim _{\tau \downarrow 0} \hat{K}_{0}^{(H)}(\tau)=\gamma+\int_{(0, \infty)} x d N(x),
$$

from which the assertion in (ii) immediately follows.

(iii) From (5.4.19) it is seen that the LT of $N$ exists on $(0, \infty)$. We calculate it from $(5.4 .16)$ with $\varphi=\hat{\xi}$, and obtain

$$
\begin{aligned}
& -\int_{(0, \infty)} e^{-\tau x} N(x) d x=\int_{(0, \infty)} e^{-\tau x} \int_{(x, \infty)} d N(y) d x= \\
& =\tau^{-1} \int_{(0, \infty)}\left(1-e^{-\tau y}\right) d N(y)=\tau^{-1}\left\{\hat{F}(\tau)^{-1}-1-\gamma \tau\right\} .
\end{aligned}
$$

(iv) This follows immediately from (iii).

(v) The assertion is a consequence of the relations (5.4.14) and (5.4.18). (vi) If $\mathrm{H}$ is the df in $F_{\mathrm{O}}$ defined by (5.4.13), then according to corollary 5.4 .7 we have $F \in F_{\infty}^{+}$iff $H \in F_{0}^{+}$. Now, by lemma 1.6 .4 it is seen that $H \in F_{0}^{+}$ iff its $K_{0}$-function $K_{0}^{(H)}$ satisfies

$$
K_{0}^{(H)}(0)=0 \text { and } \int_{(0, \infty)} \frac{1}{x} \cdot K_{0}^{(H)}(x)<\infty,
$$

which in view of $(5.4 .17)$ is equivalent to the condition that $\gamma=0$ and $N$ is bounded.

REMARK 5.4.11. From the canonical representation $(5.4 .16)$ for $\hat{F}$ it is also easily verified that if $\gamma=0$ and $N$ is bounded, then $F$ is compound-geometric$(p, G)$, with

$(5.4 .26) \quad p=-N(0+) /\{1-N(0+)\}$ and $G(x)=1-N(x) / N(0+) \quad(x>0)$.

The $\mathrm{K}_{\infty}$-function of $\mathrm{F}$ can then be expressed in $(\gamma, N)$ as follows:

$(5.4 .27) \quad K_{\infty}(x)=\{N(x)-N(0+)\} /\{1-N(0+)\} \quad(x>0)$.

Thus we see that a df $F$ in $F_{\infty}$ with $\ell(F)=0$ and for which $\gamma>0$ or $\mathbb{N}$ is unbounded, is necessarily continuous at zero, and hence is continuous every- 
where (cf. corollary 1.7.6). A further investigation of such distributions is simplified by the existence of a functional equation in terms of $\gamma$ and $N$, by means of which $F_{\infty}$ can be characterized. In fact, rewriting (5.4.21) as

$$
\hat{F}(\tau)\left\{\gamma+\int_{(0, \infty)} e^{-\tau X}\{1-N(x)\} d x\right\}=\tau^{-1} .
$$

and using the uniqueness theorem for LST's, we get the following result.

THEOREM 5.4.12. If $F$ is a df on $[0, \infty)$, then $F \in F_{\infty}$ with $l(F)=0$ iff there exist $\gamma \geq 0$ and a function $N$ satisfying the conditions for $N$ in theorem 5.4 .8 such that

$$
(5.4 .28) x-\gamma F(x)=\int_{(0, x]} F(x-y)\{1-N(y)\} d y \quad(x \geq 0) .
$$

The quantities $\gamma$ and $N$ are unique, and give the canonical representation of $F$.

We can subdivide $F_{\infty}$ in four subclasses, characterized by the following four possibilities for the canonical representation $(\gamma, N): \gamma=0$ or $\gamma>0$ and $N$ is bounded or not. The subclass $F_{\infty}^{+}=\left\{F \in F_{\infty}\right.$ with $\ell(F)=0 \mid \gamma=0, N$ bounded\} is well known. Now, using theorem 5.4.12, we can completely analyze the two classes of $\mathrm{df}^{\prime} \mathrm{s}$, for which $\gamma>0$. To this end we state the following theorem.

THEOREM 5.4.13. If $F \in F_{\infty}$, with $\ell(F)=0$, has a canonical representation $(\gamma, N)$ with $\gamma>0$, then $F$ is absolutely continuous and has a continuous density f which satisfies

$$
(5.4 .29) \quad 1-\gamma f(x)=\int_{(0, x)} f(x-y)\{1-N(y)\} d y \quad(x>0)
$$

Furthermore, this density $f$ has the following properties:

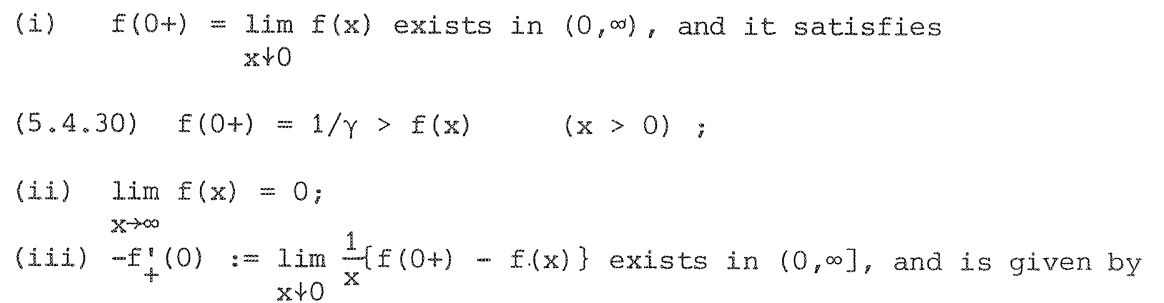


$(5.4 .31) \quad-f_{+}^{\prime}(0)=\{1-N(0+)\} / \gamma^{2} \quad(\leq \infty)$.

PROOF. Let $F \in F_{\infty}$ with $l(F)=0$. Then $F$ satisfies the functional equation (5.4.28), the right-hand side of which can be written as (cf. (5.4.19))

$$
\begin{aligned}
& \int_{[0, x]} \int_{(0, x-y]}\{1-N(z)\} \operatorname{dzdF}(y)=\int_{[0, x]} \int_{(y, x]}\{1-N(u-y)\} d u d F(y)= \\
& =\int_{(0, x][0, u)}\{1-N(u-y)\} d F(y) d u .
\end{aligned}
$$

It follows that

$$
\text { (5.4.32) } \quad \gamma F(x)=\int_{(0, x]}\{1-w(u)\} d u \quad(x \geq 0)
$$

where the function $w$ is defined on $(0, \infty)$ by

$$
(5.4 .33) w(x):=\int_{[0, x)}\{1-N(x-y)\} d F(y) \quad(x>0) .
$$

Now, suppose that $\gamma>0$. Then from (5.4.32) it is seen that $F$ is absolutely continuous; the function $f$, defined by

$$
f(x):=\{1-w(x)\} / \gamma \quad(x>0),
$$

is a density of $F$, and, because of (5.4.33), it satisfies (5.4.29). From this relation we obtain, using the monotonicity of $\mathrm{N}$,
$(5.4 .34)$
$\gamma f(x) \leq 1-\{1-N(x)\} F(x)$
$(x>0)$.

It follows that $f(x)<1 / \gamma$ for $x>0$, and, if we let $x \rightarrow \infty$, that (ii) holds. Now we can estimate as follows:

$$
0 \leq \int_{(0, x)} f(x-y)\{1-N(y)\} d y \leq \gamma^{-1}\left\{x-\int_{(0, y)} N(y) d y\right\}
$$

which, because of (5.4.19), tends to zero as $x+0$. From (5.4.29) it is now seen that $1-\gamma f(x)$ tends to zero as ' $x \downarrow 0$, and (i) is proved.

To prove the continuity of $f$, or, equivalently, of $w$, we use the following well known property:

$$
\text { (5.4.35) }\left[\forall_{x>0} \int_{(0, x]}|f(y)| d y<\infty\right] \Rightarrow\left[\forall_{a>0} \lim _{h \rightarrow 0} \int_{(0, a]}|f(x+h)-f(x)| d x=0\right] .
$$

Let $x>0$ and $h>0$, then for all $x_{0}<x$ we have 


$$
\begin{aligned}
& |w(x+h)-w(x)| \leq \int_{(0, x)}|f(x+h-y)-f(x-y)|\{1-N(y)\} d y+ \\
& +\int_{[x, x+h)} f(x+h-y)\{1-N(y)\} d y \leq 2 \gamma^{-1} \int_{\left(0, x_{0}\right)}\{1-N(y)\} d y+ \\
& +\left\{1-N\left(x_{0}\right)\right\} \int_{\left(0, x-x_{0}\right]}|f(z+h)-f(z)| d z+\{1-N(x)\} \int_{(0, h]} f(z) d z,
\end{aligned}
$$

which by (5.4.19) and $(5.4 .35)$ becomes arbitrarily small, if we choose $x_{0}$ sufficiently small. Proceeding for $h<0$ in a similar way, we conclude that $w$, and hence $f$, is continuous.

Finally, we prove (iii); since $N$ is nondecreasing, we can write for $x>0$

$$
\{1-N(x)\} F(x) \leq \int_{(0, x)} f(x-y)\{1-N(y)\} d y \leq\{1-N(0+)\} F(x) \quad(\leq \infty),
$$

from which by use of

$$
\lim _{x \downarrow 0} F(x) / x=f(0+)
$$

it follows that

$$
\begin{aligned}
& \lim _{x \ngtr 0} \frac{1}{x}\{f(0+)-f(x)\}=\gamma^{-1} \lim _{x \ngtr 0} \frac{1}{x}\{1-\gamma f(x)\}= \\
& =\gamma^{-1} \lim _{x \ngtr 0} \frac{1}{x} \int_{(0, x)} f(x-y)\{1-N(y)\} d y=\{1-N(0+)\} / \gamma^{2} \quad(\leq \infty) .
\end{aligned}
$$

From (5.4.32) we immediately obtain a characterization of $F_{\infty}^{3}$ and of $\left\{F \in F_{\infty}\right.$ wi.th $\ell(F)=0 \mid \gamma=0$ \} by a functional equation.

COROLIARY 5.4.14. A paf $f$ on $(0, \infty)$ is the density of a df $F \in F_{\infty}^{\prime}$ with $\ell(F)=0$ iff there exist $\gamma \geq 0$ and a function $N$ satisfying the conditions for $\mathrm{N}$ in theorem 5.4 .8 such that $(5.4 .29)$ holds for almost all $\mathrm{x}>0$.

COROLLARY 5.4.15. If $F$ is a df on $[0, \infty)$, then $F \in F_{\infty}$ with $\ell(F)=0$ and with $\gamma=0$ in its canonical representation (5.4.16) iff there exists a function $\mathrm{N}$ satisfying the conditions for $\mathrm{N}$ in theorem 5.4 .8 such that

(5.4.36) $\int_{[0, x)}\{1-N(x-y)\} d F(y)=1 \quad$ (almost all $x>0$ ). 
The subdivision of $F_{\infty}$ in four subclasses, mentioned just before theorem 5.4 .13 , can be characterized in terms of properties of the df's in $F_{\infty}$ themselves; this is easily verified from the preceding theorem and the following lemma.

LEMMA 5.4.16. If a df $F \in F_{\infty}$, with $\ell(F)=0$ and canonical representation $(\gamma, N)$, is absolutely continuous and has a density $f$, for which $f(0+)$ exists in $[0, \infty]$, then necessarily $f(0+) \in(0, \infty]$, and

(i) $f(0+) \in(0, \infty)$ iff $\gamma>0$, in which case $f(0+)=1 / \gamma$;

(ii) $f(0+)=\infty$ iff $\gamma=0$, in which case $N$ is unbounded.

PROOF. If a df $F$ on $[0, \infty)$ has a density $f$, for which $f(0+)$ exists in $[0, \infty]$, then, as is easily verified, $f(0+)$ can be obtained as follows:

$$
f(0+)=\lim _{\tau \rightarrow \infty} \tau \hat{F}(\tau)
$$

Now, if $F \in F_{\infty}^{\prime}$ with $\ell(F)=0$, then in view of lemma 5.4.10(iv) it is seen that $f\left(0^{+}\right)$cannot be zero, and that $f(0+)<\infty$ iff $\gamma>0$, in which case $f(0+)=1 / \gamma$. Finally, if $f(0+)=\infty$, then $\gamma=0$ and hence $N$ is unbounded, as otherwise by lemma 5.4.10(vi) we would have $F \in F_{\infty}^{+}$.

THEOREM 5.4.17. Let $F$ be in $F_{\infty}$ with $\ell(F)=0$ and canonical representation $(\gamma, N)$. Then the following four cases can be distinguished:

(i) $\gamma=0$ and $N$ is bounded iff $F$ is compound geometric $\left(F \in F_{\infty}^{+}\right)$:

(ii) $\gamma>0$ and $N$ is bounded iff $F$ is absolutely continuous and has a density $f_{\text {, for which }} f\left(O_{+}\right)$and $-f_{+}^{\prime}(0)$ exist in $(0, \infty)$;

(iii) $\gamma>0$ and $N$ is unbounded iff $F$ is absolutely continuous and has a den-sity $f$, for which $f(0+)$ exists in $(0, \infty)$ and $-f_{+}^{*}(0)=\infty$;

(i.v) $\gamma=0$ and $N$ is unbounded iff $F$ is continuous and $F$ is either not absolutely continuous, or absolutely continuous such that no density of $F$ has a finite limit as $x * 0$.

The following lemma provides a method to construct examples of distributions in each of the four subclasses of $F_{\infty}$ from given inf div distributions on $[0, \infty)$.

LEMMA 5.4.18. If $\mathrm{H} \in F_{O^{\prime}}$ then $\{\dot{1}-\log \hat{\mathrm{H}}(\tau)\}^{-1}$ is the PLST $\hat{\mathrm{F}}$ of a df $F \in F_{\infty}$ with $\ell(F)=0$, and the canonical representation $(\gamma, N)$ of $F$ satisfies 
(i) $\gamma>0$ iff $\ell(\mathrm{H})>0$;

(ii) $N$ is bounded iff $H(\ell(H))>0$.

PROOF. The first part of the lemma follows fxom theorem 5.4.5. Further, from corollary 5.4.9 and lemma 1.6 .4 it is seen that $\gamma=K_{0}^{(\mathrm{H})}(0)=\ell(\mathrm{H})$ and that $\mathrm{N}$ is bounded iff

$$
\int_{(0, \infty)} \frac{1}{x} d K_{0}^{(H)}(x)<\infty
$$

i.e. iff $H(\ell(H))>0$.

Just as $F_{\infty}^{+}(\mathrm{cf} .(5.4 .1))$, the subclass $\left\{F \in F_{\infty}\right.$ with $\ell(F)=0 \mid \gamma>0, N$ is bounded $\}$ is dense in $\left\{F \in F_{\infty} \mid \ell(F)=0\right\}$ in the sense of weak convergence; in fact, if $F \in F_{\infty}$ with $\ell(F)=0$ and canonical representation $(\gamma, N)$, then by the monotone convergence theorem it is seen that $\hat{F}(\tau)=\lim _{n \rightarrow \infty} \hat{F}_{n}(\tau)$, where $\mathrm{F}_{\mathrm{n}}(\mathrm{n} \in \mathbb{N})$ is defined as the df with PLST (5.4.16) with $\gamma$ and $\mathrm{N}$ replaced by

$$
\gamma_{n}:=\left\{\begin{array}{ll}
\gamma_{0} & \text { if } \gamma>0 \\
1 / n, & \text { if } \gamma=0
\end{array} ; N_{n}(x):= \begin{cases}N(x), & \text { if } N \text { bounded or } x \geq 1 / n \\
N(1 / n), & \text { otherwise. }\end{cases}\right.
$$

Furthermore, this subclass of $F_{\infty}$ turns out to consist of convolutions of an exponential distribution and a compound-geometric- $(p, G)$ one, where $G$ has the same exponential distribution as a factor. We state this in the following theorem, and note that distributions of this type also occur as first-passage time distributions in Miller (1967).

THEOREM 5.4.19. If $F$ is a df on $[0, \infty)$, then $F \in F_{\infty}$ with $\ell(F)=0$ and having a canonical representation $(\gamma, N)$ with $\gamma>0$ and $N$ bounded iff $\hat{F}$ has the form

$(5.4 .37) \hat{F}(\tau)=\frac{\mu}{\mu+\tau} \frac{1-p}{1-p\{\mu /(\mu+\tau)\} G(\tau)}$ where $\mu>0,0 \leq p<1$ and $G$ is a df on $[0, \infty)$.

PROOF. Let the PLST $\hat{F}$ of a df $F$ on $[0, \infty)$ have the form $(5.4 .16)$ with $\gamma>0$ and $\mathrm{N}$ bounded. Then, defining

$(5.4 .38) \mu:=\{1-N(0+)\} / \gamma, p:=-N(0+) /\{1-N(0+)\}, G(x):=1-N(x) / N(0+)$, we see that $\hat{F}$ takes the form $(5,4.37)$. 
Conversely, the $\psi_{\infty}$-function of a df F with PLST given by (5.4.37) satisfies

$$
\begin{aligned}
\psi_{\infty}(\tau) & =\frac{d}{d \tau} \hat{F}(\tau)^{-1}=\frac{d}{d \tau}\left[1+\{\mu(1-p)\}^{-1} \tau+\{p /(1-p)\}(1-\hat{G}(\tau))\right]= \\
& =\{\mu(1-p)\}^{-1}-\{p /(1-p)\} \hat{G}^{\prime}(\tau)
\end{aligned}
$$

which is comp mon. Hence by theorem 5.4.2 F $\in F_{\infty}$ with $\ell(F)=0$, and because of corollaries 5.4 .6 and 5.4 .9 it follows that the canonical representation is given by

(5.4.39) $\gamma=\{\mu(1-p)\}^{-1}, N(x)=-\{p /(1-p)\}(1-G(x)) \quad(x>0)$, so $\gamma>0$ and $N$ is bounded.

EXAMPLE 5.4.20. In $(5.4 .37)$ take $\hat{G}(\tau)=\frac{\nu}{\nu+\tau} / \frac{\mu}{\mu+\tau}$ with $0<\nu \leq \mu$; by example 5 on p. 153, this is indeed a PLST (in $F_{\infty}^{+}$). Then it follows that

$$
\frac{\mu}{\mu+\tau} \frac{\nu(1-p)}{\nu(1-p)+\tau} / \frac{\nu}{\nu+\tau} \in F_{\infty}^{\prime}
$$

However, we can prove a little more: if $\mu_{1}, \mu_{2}$ and $\mu_{3}$ are all positive, then $(5.4 .40) \quad \hat{\mathrm{F}}(\tau):=\frac{\mu_{1}}{\mu_{1}+\tau} \frac{\mu_{3}}{\mu_{3}+\tau} / \frac{\mu_{2}}{\mu_{2}+\tau} \epsilon F_{\infty}^{8} \Leftrightarrow \min \left(\mu_{1}, \mu_{3}\right) \leq \mu_{2} \leq \max \left(\mu_{1}, \mu_{3}\right)$. First we note that by lemma $5.3 .10 \hat{\mathrm{F}}$ is indeed a PLST iff $\mu_{2} \geq \min \left(\mu_{1}, \mu_{3}\right)$. Calculating then the $\psi_{\infty}$-function of $F$, we get

$$
\psi_{\infty}(\tau)=1+\left(\mu_{1}-\mu_{2}\right)\left(\mu_{2}-\mu_{3}\right)\left(\mu_{2}+\tau\right)^{-2}
$$

which is comp mon iff $\left(\mu_{1}-\mu_{2}\right)\left(\mu_{2}-\mu_{3}\right) \geq 0$, i.e. iff $\mu_{2} \leq \max \left(\mu_{1}, \mu_{3}\right)$. Final$I y$, we note that under these conditions $\hat{\mathrm{F}}$ is, in fact, a mixture of two exponential PLST's:

$$
\hat{\mathrm{F}}(\tau)=\left\{\mu_{2}\left(\mu_{3}-\mu_{1}\right)\right\}^{-1}\left\{\mu_{3}\left(\mu_{2}-\mu_{1}\right) \frac{\mu_{1}}{\mu_{1}+\tau}+\mu_{1}\left(\mu_{3}-\mu_{2}\right) \frac{\mu_{3}}{\mu_{3}+\tau}\right\} .
$$

Hence (cf. lemma 1.6.10) F has a comp mon density and therefore belongs to $F_{\infty}^{\prime}$, as we shall see presently.

The subclass $\left\{F \in F_{\infty}\right.$ with $\ell(F)=0 \mid \gamma=0, N$ unbounded $\}$ of $F_{\infty}$ is still rather obscure. Apart from PLST'S of the form $\{1-\log \hat{\mathrm{H}}(\tau)\}^{-1^{\infty}}$ with $\mathrm{H} \in F_{\text {。 }}$ and $\ell(\mathrm{H})=H(\ell(H))=0$ ( Cf. lemma 5.4.18), it contains all pdf's $f \in F_{\infty}^{\prime}$ ' for which $f(0+)=\infty$ (cf. lemma 5.4.16(ii)). Beforegiving some examples, we state an analogue of corollary 5.2.8. 
THEOREM 5.4.21. If $F \in F_{\infty}$ with $\ell(F)=0$ and with canonical representation $(\gamma, N)$ such that $\gamma=0$ and $N$ is unbounded and absolutely continuous, then $F$ is absolutely continuous.

PROOF. If $\gamma=0$ and $N$ is absolutely continuous with density $n$, then from (5.4.23) it is seen that the $K_{0}$-function of $F$ is absolutely continuous with density $k_{0}$ given by

$$
k_{0}(x)=\int_{[0, x)}(x-y) n(x-y) d F(y) \quad(x>0) .
$$

Now, by theorem 5.2 .7 it follows that $F(x)-F(0)$ is absolutely continuous. If in addition $\mathrm{N}$ is unbounded, then necessarily $F(0)=0$, and hence $F$ is absolutely continuous, with density $f$ satisfying (cf. (5.2.19))

(5.4.41) $\operatorname{xf}(x)=\int_{(0, x]} f^{* 2}(x-y) y n(y) d y \quad(x>0)$.

REMARK 5.4.22. Also from (5.4.23) it is seen that, contrary to the case of a general inf div df in $F_{0}^{\prime \prime}$ (cf. remark 5.2.9), the $k_{0}$-function of a df $F \in F_{\infty}^{8}$ with $\ell(F)=0$ is necessarily absoiutely continuous. It follows that corollary 5.2.8 gives necessary and sufficient conditions in terms of the $\mathrm{K}_{\mathrm{O}}$-function for $\mathrm{F} \in \mathrm{F}_{\infty}$ to be absolutely continuous. However, such conditions are not easily obtained in terms of the function $\mathrm{N}$.

EXAMPLE 5.4.23.

(i) Consider the gamma distribution with parameters $\mu>0$ and $\nu>0$ :

$$
f(x)=\frac{\mu^{\nu}}{\Gamma(\nu)} x^{\nu-1} e^{-\mu x}(x>0), \text { or } \hat{F}(\tau)=\left(\frac{\mu}{\mu+\tau}\right)^{\nu} \text {. }
$$

By (5.3.13) we know that if $0<\nu \leq 1$ then $f \in F_{\infty}^{*}$. If $\nu<1$ then $f(0+)=\infty$, and hence in that case: $\gamma=0$ and $N$ unbounded. Indeed, the $\psi_{\infty}$-function of $f$ is given by

$$
\psi_{\infty}(\tau)=\frac{\nu}{\mu}\left(\frac{\mu}{\mu+\tau}\right)^{1-\nu}
$$

and hence (cf. corollaries 5.4 .6 and 5.4.9)

$$
\gamma=0 \text { and } N(x)=-\frac{\nu^{--\nu}}{\Gamma(1 \cdot-\nu)} \int_{(x, \infty)} y^{-1-\nu} e^{-\mu y} d y \quad(x>0) .
$$


(ii) Take for $\mathrm{H}$ in lemma 5.4 .18 a gamma distribution. Then it follows that

$$
\hat{\mathrm{F}}(\tau):=\{1+\nu \log (1+\rho \tau)\}^{-1} \in F_{\infty}^{\prime} \quad(\nu>0 ; \rho>0),
$$

with $\gamma=0$ and $N$ unbounded. Here, $F$ is absolutely continuous, because $N$ is: the $\psi_{\infty}$-function of $F$ is given by

$$
\psi_{\infty}(\tau)=\rho \nu /(1+\rho \tau)
$$

and hence

$$
N(x)=-v \int_{(x, \infty)} y^{-1} e^{-p^{-1} y} d y \quad(x>0) .
$$

(i.i.) Take $0<\alpha<1$, and consider the function $N$ on $(0, \infty)$, defined by

$$
N(x):=\left(1-x^{-\alpha}\right) 1(0,1]^{(x)} \quad(x>0) .
$$

Then $\mathrm{N}$ is absolutely continuous and satisfies the conditions in theorem 5.4 .8 , so that

$$
\text { (5.4.42) } \quad \hat{\mathrm{F}}(\tau):=\left\{1+\alpha \int_{(0,1]}\left(1-\mathrm{e}^{-\tau \mathrm{Tx}}\right) \mathrm{x}^{-\alpha-1-1} \mathrm{dx}\right\}^{-1} \in F_{\infty}^{8}
$$

in the canonical representation $(\gamma, N)$ of $F, \gamma=0$ and $N$ is unbounded. The PLST (5.4.42) occurs in Feller (1971), ch. XIII (problem section) as the limiting PLST of the distribution of $s_{n} / M_{n}$, where $s_{n}:=x_{1}+\ldots+x_{n}$ $M_{n}:=\max \left\{x_{1}, \ldots, x_{n}\right\}$ and $x_{1}, x_{2}, \ldots$ are independent $r v^{\prime} s$ with common distribution belonging to the domain of attraction of the stable distribution on $[0, \infty)$ with exponent $\alpha$.

After having studied the structure and basic properties of $F_{\infty}$, we turn to analogues of some of the properties of $C_{O^{\prime}}$ mentioned in theorems 1.5 .13 and 2.4 .9 , for $F_{\infty}$.

As noted at the end of section 1.6, Goldie (1967) and steutel (1970) prove that the classes $D$ and $E$ of comp mon and log-convex pdf's, respectively, are subclasses of $F_{0}$. We now prove that they are subclasses of $F_{\infty}$, i.e. (5.4.43) $D \subset E \subset F_{\infty}$.

THEOREM 5.4.24. If $f$ is a log-convex pdf on $(0, \infty)$, then $f \in F_{\infty}^{\prime}$. 
PROOF. Let $F$ be a df on $[0, \infty)$ with a log-convex density $f$. Then for every $\mathrm{h}>0$ there exists $\mathrm{c}_{\mathrm{h}}>0$ such that the sequence $\left\{\mathrm{p}_{\mathrm{n}}^{(\mathrm{h})}\right\}_{0}^{\infty}$, defined by

$$
\mathrm{p}_{\mathrm{n}}^{(\mathrm{h})}:=\mathrm{c}_{\mathrm{h}} \mathrm{hf}\left(\frac{1}{2}(2 \mathrm{n}+1) \mathrm{h}\right) \quad\left(\mathrm{n} \in \mathbb{N}_{0}\right)
$$

satisfies $\sum p_{n}^{(h)}=1$. As $f$ is a log-convex function, $\left\{p_{n}^{(h)}\right\}$ is a log-convex sequence, and hence (cf. theorem 1.5.13) satisfies the recurrence relations (1.5.17) with nonnegative $r_{n}(0)$ 's. Now, consider $\left\{p_{n}^{(h)}\right\}$ as a probability distribution on the lattice $\{0, h, 2 h, \ldots\}$. Then by (5.1.9) with $\alpha=0$ it follows that $\left\{\mathrm{p}_{\mathrm{n}}^{(\mathrm{h})}\right\} \in \mathrm{F}_{\infty}^{+}$for all $\mathrm{h}>0$. Since furthermore

$$
F(x)=\lim _{h \downarrow 0} \sum_{n=0}^{[x / h]} h f\left(\frac{1}{2}(2 n+1) h\right)=\lim _{h \downarrow 0} \sum_{n h \leq x} p_{n}^{(h)} \quad(x \geq 0),
$$

and as $F_{\infty}$ is closed under weak convergence (cf. theorem 5.3.1), we conclude that $F \in F_{\infty}$.

COROLLARY 5.4.25. If $f$ is a comp mon pdf on $(0, \infty)$, then $f \in F_{\infty}^{\prime}$. EquivalentIy (cf. lemma 1.6 .10 ), if $G$ is a df on $(0, \infty)$, then the PLST

$$
(5.4 .44) \quad \int_{(0, \infty)} \frac{\mu}{\mu+\tau} \mathrm{dG}(\mu) \in F_{\infty}^{\prime} .
$$

Steutel (1970) proves the following implication:

$(5.4 .45) \quad F \in D \Rightarrow \forall p \in(0,1) p+(1-p) \hat{F}(\tau) \in F_{0}^{+}$

We can improve on this result as follows.

THEOREM 5.4.26. If $F \in F_{\infty}$ with $\ell(F)=0$ and if $0<p<1$, then the PLST

$$
p+(1-p) \hat{F}(\tau) \in F_{\infty}^{+} \text {. }
$$

PROOF. We calculate the $\varphi_{\infty}$-function of $p+(1-p) \hat{F}$, and obtain (5.4.46) $\varphi_{\infty}(\tau)=1-\frac{p+(1-p) F(0)}{p+(1-p) \hat{F}(\tau)}=(1-p)\left\{1-\frac{F(0)}{\hat{F}(\tau)}\right\} \frac{\hat{F}(\tau)}{p+(1-p) \hat{F}(\tau)}$.

Denoting the last factor in the right-hand side of $(5.4 .46)$ by $\varphi(\tau)$, we can write $\varphi(\tau)=\{1-\log \hat{\mathrm{H}}(\tau)\}^{-1}$, where according to corollary 5.4 .6 and theorem 1.4 .4

$$
\hat{\mathrm{H}}(\tau):=\exp \left[\mathrm{p}\left(1-\hat{\mathrm{F}}(\tau)^{-1}\right)\right]
$$


is the PLST of a df $\mathrm{H}$ in $F_{0}$. Now, by theorem 5.4 .5 it follows that $\varphi$ is the PLST of a df in $F_{\infty}$. Hence $\varphi$ is comp mon, and applying lemma 5.1.4(ii) once in each direction, we conclude from (5.4.46) that $p+(1-p) \hat{F} \in F_{\infty}^{+}$.

COROLLARY 5.4.27. If $G$ is a df on $(0, \infty)$, and if $0<p<1$, then the PLST $(5.4 .47) p+(1-p) \int_{(0, \infty)} \frac{\mu}{\mu+\tau} d G(\mu) \in F_{\infty}^{+}$.

REMARK 5.4.28. Starting with continuous df's $\mathrm{F}$ in theorem 5.4.26, we cannot generate all compound geometric PLST's. In fact, in view of the result to be given in corollary 5.4.30, it will be clear that for $\mathrm{p} \in(0,1)$ a PLST $\hat{\mathrm{F}}$ has the form $1-p+p \hat{H}(\tau)$, where $H$ is a continuous df in $F_{\infty}$ with $\ell(H)=0$, iff $F$ is compound-geometric- $(p, G)$ with $G \in F_{\infty}, l(G)=0$.

The function $\varphi$ from the proof of theorem 5.4.26 and the tot-dec(1) pgf's in section 3.4 suggest another closure property of $F_{\infty}$.

THEOREM 5.4.29. Let $F$ be a df on $[0, \infty)$, and define for $\mu>0$ the function $\psi_{\mu}$ by

$(5.4 .48) \quad \psi_{\mu}(\tau):=\frac{\mu}{\mu-1+\hat{F}(\tau)^{-1}} \quad(\tau \geq 0)$.

Then:

(i) $\psi_{\mu}$ is a PLST for all $\mu \in(0,1]$.

(ii) If $\psi_{\mu}$ is a PLST, then $\psi_{\lambda}$ is a PLST for all $\lambda \in(0, \mu]$.

(iii) $\psi_{\mu}$ is a PLST for all $\mu>0$ iff $F \in F_{\infty}$ with $l(F)=0$, in which case for all $\mu>0$ the df $F_{\mu}$, with $\hat{\mathrm{F}}_{\mu}=\psi_{\mu}$, is also in $F_{\infty}$ with $\ell\left(F_{\mu}\right)=0$.

PROOF. Since $\psi_{\mu}$ can be written in the form

$$
\psi_{\mu}(\tau)=\frac{1-(1-\mu)}{1-(1-\mu) \hat{F}(\tau)} \hat{\mathbb{F}}(\tau) \quad(\tau \geq 0),
$$

it is seen that $\psi_{\mu}$ is a PLST for $0<\mu \leq 1$. Using the relation

$$
\hat{F}(\tau)=\frac{\psi_{\mu}(\tau)}{\mu+(1-\mu) \psi_{\mu}(\tau)},
$$

we obtain the following relation between $\psi_{\lambda}$ and $\psi_{\mu}$ : 


$$
\psi_{\lambda}(\tau)=\frac{\lambda / \mu}{1-(1-\lambda / \mu) \psi_{\mu}(\tau)} \psi_{\mu}(\tau)
$$

from which (ii) follows.

The "if" part of (iii) follows from the proof of theorem 5.4.26, where we

showed that if $F \in F_{\infty}$ with $\ell(F)=0$ then $\psi_{\mu}$ is a PLST $\hat{F}_{\mu}$ in $F_{\infty}$ with $\ell(F)=0$ for all $\mu>0$.

Therefore, suppose that $\psi_{\mu}$ is a PLST $\hat{\mathrm{F}}_{\mu}$ for all $\mu>0$. Rewrite $\hat{\mathrm{F}}_{\mu}$ as

$$
\hat{\mathrm{F}}_{\mu}(\tau)=\left\{1+\mu^{-1}\left[\hat{\mathrm{F}}(\tau)^{-1}-1\right]\right\}^{-1} \quad(\tau \geq 0),
$$

and let $\gamma>0$. Then, taking $\mu_{n}:=n / \gamma(n \in \mathbb{N})$, we see that the PLST $\left\{\hat{\mathrm{F}}_{\mu_{\mathrm{n}}}(\tau)\right\}^{\mathrm{n}}$ satisfies

$$
\hat{\mathrm{H}}_{\gamma}(\tau):=\lim _{n \rightarrow \infty}\left\{\hat{\mathrm{F}}_{\mu_{n}}(\tau)\right\}^{\mathrm{n}}=\exp \left[\gamma\left(1-\hat{F}^{\hat{*}}(\tau)^{-1}\right)\right] .
$$

As $\hat{\mathrm{H}}_{\gamma}(0+)=1$, it follows by the continuity theorem for LST's that $\hat{\mathrm{H}}_{\gamma}$ is a PLST for all $\gamma>0$, and hence $\hat{\mathrm{H}}_{1}$ is an inf div PLST. From theorem 5.4 .5 we now conclude that $F \in F_{\infty}$ with $\ell(F)=0$.

COROLLARY 5.4.30. Let $F$ be a df on $[0, \infty)$, and let $0<p<1$. Then $F \in F_{\infty}$ with $\ell(F)=0$ iff the following PLST $\hat{G}$ is in $F_{\infty}$ with $\ell(G)=0$ :

$(5.4 .49) \quad \hat{G}(\tau):=\frac{1-p}{1-\hat{p} \hat{F}(\tau)} \hat{F}(\tau) \quad(\tau \geq 0)$.

Steutel. (1970) has similar results for chf's. For instance, he gives an analogue of theorem 5.4.29(i); furthermore, he obtains a result (stated in the following theorem) that immediately yields also an analogue of theorem 5.4.29(iii) for chf's.

THEOREM 5.4.31. Let $\psi$ be a function on $\mathbb{R}$. Then the function $\psi_{\mu}$, defined by

$$
\psi_{\mu}(t):=\frac{\mu}{\mu+\psi(t)} \quad(t \in \mathbb{R}),
$$

is a chf for all $\mu>0$ iff $\psi(t)=-\log \tilde{H}(t)$ with $\mathrm{H}$ an inf div df on $\mathbb{I R}$.

COROLLARY 5.4.32. Let $F$ be a df on $\mathbb{I R}$. Then the function $\psi_{\mu}$, defined by

$$
\psi_{\mu}(t):=\frac{\mu}{\mu-1+\tilde{F}(t)^{-1}} \quad(t \in \mathbb{R}),
$$

is a chf for all $\mu>0$ iff $\tilde{F}(t)=\{1-\log \tilde{H}(t)\}^{-1}$ with $H$ an inf div df on $\mathbb{R}$. 
The preceding theorems provide examples of distributions in $F_{\infty}$. We mention a few of them.

EXAMPLE 5.4.33.

(i) Because of theorem 5.4.26 we have (cf. example 5, p. 153)

$$
\frac{\nu}{\nu+\tau} / \frac{\mu}{\mu+\tau}=\frac{\nu}{\mu}+\left(1-\frac{\nu}{\mu}\right) \frac{\nu}{\nu+\tau} \in F_{\infty}^{+} \quad(0<\nu<\mu) .
$$

(ii) Scale mixtures of gamma distributions with (fixed) second parameter $\nu \in(0,1]$ can be regarded as mixtures of exponential distributions (cf. steutel (1970)); hence they are in $F_{\infty}^{\prime}$. For instance

$$
\int_{(1, \infty)}\left(\frac{\mu}{\mu+\tau}\right) \nu_{\mu}^{-2} d \mu=\frac{(1+\tau)^{1-\nu}-1}{(1-\nu) \tau} \in F_{\infty}^{8} \quad(0<\nu<1),
$$

and hence (take $\nu=\frac{1}{2}$, and apply theorems 5.4.29(iii) and 5.4.26)

$$
(5.4 .50) \quad p+(1-p) \frac{r+1}{r+(1+\tau)^{\frac{1}{2}}} \in F_{\infty} \quad(0 \leq p<1 ; x>-1) .
$$

(i.ii) The density $f_{x}$ of $x^{r}$, where $x$ has a gamma distribution with parameters $\mu>0$ and $\nu>0$, satisfies

$(5.4 .51) \quad f_{x}(x)=\frac{\mu^{\nu}}{x \Gamma^{\prime}(\nu)} x^{\nu / x-1} \exp \left[-\mu x^{1 / r}\right] \in F_{\infty}^{\prime} \quad(\mu>0, \nu>0 ; x \geq \max (1, \nu))$, as it is $\log$-convex iff $r \geq \max (1, \nu)$.

In the next theorem we state a property of $F_{\infty}$ that can be viewed as an analogue of the following closure property of $F_{0}$ (cf, theorem 1.4.9):

(5.4.52) $\left[G \in F_{0^{\prime}} H \in F_{0}\right] \Rightarrow \hat{G}(-\log \hat{H}(\tau))$ is a PLST in $F_{0}$. Here, the inf div of $\mathrm{H}$ ensures the function $\hat{\mathrm{G}}(-\log \hat{\mathrm{H}}(\tau))$ to be a PLST.

THEOREM 5.4.34. If $G \in F_{\infty}$ with $\ell(G)=0$, and if $H \in F_{O^{\prime}}$ then the function (5.4.53) $\hat{G}(-\log \hat{H}(\tau))=\int_{[0, \infty)} \hat{H}(\tau)^{x} \mathrm{dG}(x) \quad(\tau \geq 0)$ is a PLST in $F_{\infty}$ with left extremity equal to zero. 
PROOF. AS $H \in F_{O^{\prime}}$ the function $\hat{G}(-\log \hat{H}(\tau))$ is a PLST. Its $\psi_{\infty}$-function is given by

$$
\begin{aligned}
\psi_{\infty}(\tau) & =\frac{d}{d \tau}\{\hat{G}(-\log \hat{H}(\tau))\}^{-1}= \\
& =-\left.\{\hat{G}(-\log \hat{H}(\tau))\}^{-2} \varphi_{0}^{(H)}(\tau) \frac{d}{d \sigma} \hat{G}(\sigma)\right|_{\sigma=-\log \hat{H}(\tau)}= \\
& =\varphi_{0}^{(H)}(\tau) \psi_{\infty}^{(G)}(-\log \hat{H}(\tau)) .
\end{aligned}
$$

The $\varphi_{0}$-function $\varphi_{0}^{(H)}$ of $H$ is comp mon, as $H \in F_{0}$. Since $G \in F_{\infty}$ with $\ell(G)=0$, its $\psi_{\infty}$-mfunction $\psi_{\infty}^{(G)}$ is comp mon (cf. theorem 5.4.2), and as - $\log \hat{H}(\tau)$ is nonnegative and has a comp mon derivative, we conclude from lemma 1.3.8(vi) that $\psi_{\infty}^{(G)}(-\log \hat{\mathrm{H}}(\tau))$ is comp mon. It follows that $\psi_{\infty}$ is comp mon, and the theorem is proved.

In view of theorem 5.4 .5 and corollary $5.4 .6,(5.4 .52)$ and theorem 5.4 .34 can be reformulated as follows.

COROLLARY 5.4 .35$.

(i) If $G \in F_{0}$ and if $H \in F_{\infty}$ with $\ell(H)=0$, then $\hat{G}\left(\hat{\mathrm{A}}(\tau)^{-1}-1\right.$ ) is a PLST in F.

(ii) If $G \in F_{\infty}$ with $\ell(G)=0$, and if $H \in F_{\infty}$ with $\ell(H)=0$, then $\hat{G}\left(\hat{H}(\tau)^{-1}-1\right)$ is a PLST in $F_{\infty}$.

REMARK 5.4.36. If $G$ is the df corresponding to a lattice distribution with pgf $\mathrm{P}$ and $\mathrm{P}(0)>0$, then in $(5.4 .52)$ and theorem 5.4 .34 i.t is not necessary to require the inf div of $\mathrm{H}$; in fact, for all df's $\mathrm{H}$ on $[0, \infty)$

$(5.4 .54) \quad \hat{G}(-\log \hat{H}(\tau))=P(\hat{H}(\tau))$

is the PLST of a compound distribution, and hence is in $F_{0}^{+}$or $F_{\infty}^{+}$according as $P \in C_{1}$ or $P \in C_{0}$ (cf. lemma 1.4.14). Further, in view of (5.4.54), also for a non-lattice df $G$ on $[0, \infty)$, but with an inf div df $A, \hat{G}(-\log \tilde{H}(t))$ could be called the chf of a compound distribution. Since every $\hat{F} \in F_{\infty}$ with $\ell(F)=0$ can be written in the form $\hat{G}(-\log \hat{\mathrm{H}}(\tau))$ with $\hat{G}(\tau)=(1+\tau)^{-1}$ (cf. theorem 5.4.5), it then follows that the distributions in $F_{\infty}$ with left extremity equal to zexo can be regarded as "compound exponential" distributions on $[0, \infty)$. Similarly, the chf"s $\psi_{\mu}$, considered in theorem 5.4.31, correspond to "compound exponential" distributions on IR. 
To conclude this section, we return to functional equations. For $\lambda<\infty$ the $F_{\lambda}$ 's have been defined by means of the equation

(5.4.55) $\int_{[0, x]} c(y ; \lambda) d F(y)=\int_{[0, x]} F(x-y) d K_{\lambda}(y) \quad(x>0)$,

with a nondecreasing function $\mathrm{k}_{\lambda}$. Letting $\lambda \rightarrow \infty$, we get (cf. theorem 1.6.7) the characterization of the class $F_{\infty}^{+}$by

$(5.4 .56) \quad F(x)-F(0)=\int_{[0, x]} F(x-y) d K_{\infty}(y) \quad(x>0)$,

with a nondecreasing function $\mathrm{K}_{\infty}$ "

Now, we want to know how from $(5.4 .55)$ or $(5.4 .56)$ the functional equation can be derived, by means of which $F_{\infty}$ has been characterized (cf. theorem $5.4 .12)$ :

$(5.4 .57) x-\gamma F(x)=\int_{(0, x]} F(x-y)\{1-N(y)\} d y \quad(x>0)$,

where $\gamma \geq 0$ and $N$ is a nondecreasing function with $N(\infty)=0$ and

$\int \mathrm{xdN}(\mathrm{x})<\infty$.

$(0,1)$

First, let us consider the case that $F \in F_{\infty}^{+}$. Then $\gamma=0$ and $N$ is bounded, and from remark 5.4 .11 it is seen that

$(5.4 .58) \quad 1-K_{\infty}(x)=F(0)\{1-N(x)\} \quad(x>0)$.

Rewriting $(5.4 .56)$ as

$$
F(0)=F(x)-\int_{[0, x]} F(x-y) d K_{\infty}(y) \quad(x>0),
$$

and integrating this equation over $[0, z]$, we get

$$
F(0) z=\int_{[0, z]} F(z-y)\left\{1-K_{\infty}(y)\right\} d y \quad(z>0),
$$

which by $(5.4 .58)$ is equivalent to $(5.4 .57)$ with $\gamma=0$.

When considering a general $F \in F_{\infty}$, we need the first relation in the following lemma.

LEMMA 5.4.37. Let $F \in F_{\infty}$ with $l(F)=0$. Then the following relations hold between the canonical representation $(\gamma, N)$ and the $K_{\lambda}$-functions of $F$ : 
(5.4.59) $\lim _{\lambda \rightarrow \infty} \frac{1}{\bar{F}(\lambda)} \int_{[0, x]}\left\{1-c_{\lambda}^{-1} K_{\lambda}(y)\right\} d y=\gamma+\int_{(0, x]}\{1-N(y)\} d y$,

(5.4.60) $\lim _{\lambda \rightarrow \infty} \frac{1}{\hat{F}(\lambda)} \int_{[0, x]} y_{d x}(y)=\gamma+\int_{(0, x]} y d N(y)$

(5.4.61) $\lim _{\lambda \rightarrow \infty} \frac{1}{\hat{\mathrm{F}}(\lambda)}\left\{1-\mathrm{C}_{\lambda}^{-1} \mathrm{~K}_{\lambda}(\mathrm{x})\right\}=1-\mathrm{N}(\mathrm{x})$.

PROOF. In view of $(5.4 .1)$ and $(5.4 .21)$ we have the following relation:

(5.4.62) $\lim _{\lambda \rightarrow \infty} \frac{1}{F(\lambda)} \tau^{-1}\left\{1-c_{\lambda}^{-1} \bar{K}{ }_{\lambda}(\tau)\right\}=\gamma+\int_{(0, \infty)} e^{-\tau x}\{1-N(x)\} d x$

from which by the continuity theorem for LST's (cf. Feller (1971), ch. XIII) (5.4.59) follows.

By the canonical representation $(5.4 .16)$ for $\hat{\mathrm{F}}$ we can rewrite $(5.4 .4)$ in the form

(5.4.63) $\lim _{\lambda \rightarrow \infty}-\frac{\mathrm{d}}{\mathrm{d} \tau} \hat{\mathrm{K}}_{\lambda}(\tau) / \hat{\mathrm{F}}(\lambda)=\gamma+\int_{(0, \infty)} e^{-\tau x} \mathrm{xdN}(\mathrm{x})$.

Applying the continuity theorem once more, we get (5.4.60).

Finally, rewriting the integrals in $(5.4 .59)$ as

$$
\int_{[0, x]}\left\{1-c_{\lambda}^{-1} K_{\lambda}(y)\right\} d y=x\left\{1-c_{\lambda}^{-1} K_{\lambda}(x)\right\}+c_{\lambda}^{-1} \int_{[0, x]} y d K_{\lambda}(y)
$$

and $\left(c f_{0}(5.4 .24)\right)$

$$
\int_{(0, x]}\{1-N(y)\} d y=x\{1-N(x)\}+\int_{(0, x]} y d N(y)
$$

respectively, and using (5.4.59) and (5.4.60), we obtain (5.4.61).

Now, we are ready to derive (5.4.57) from (5.4.55). Rewrite (5.4.55) as

$$
\int_{[0, x]} e^{-\lambda y} d F(y)=F(x)-c_{\lambda}^{-1} \int_{[0, x]} F(x-y) d K_{\lambda}(y),
$$

then, integrating this equation over $[0, z]$, we get

$$
\int_{[0, z]}(z-y) e^{-\lambda y} d F(y)=\int_{[0, z]} F(z-y)\left\{1-c_{\lambda}^{-1} K_{\lambda}(y)\right\} d y,
$$


or, dividing by $\hat{F}(\lambda)$ and rewriting the left-hand side,

$$
z-\frac{1}{\bar{F}(\lambda)}\left\{z \int_{(z, \infty)} e^{-\lambda y} d F(y)+\int_{[0, z]} y e^{-\lambda y} d F(y)\right\}=
$$

$(5.4 .64)$

$$
=\int_{[0, z]} F(z-y) \frac{1-c_{\lambda}^{-1} K_{\lambda}(y)}{\hat{F}(\lambda)} d y .
$$

Letting $\lambda \rightarrow \infty$, and using (5.4.59) and the fact that the absolute value of the second term in the left-hand side of $(5.4 .64)$ is less than $-\hat{F}^{\circ}(\lambda) / \hat{F}(\lambda)$, and hence tends to zero (cf. lemma 5.1.6), we get the functional equation $(5.4 .57)$.

\subsection{The class $F_{\infty}$ in relation to standard $p$-functions}

The relation between $C_{O}$ and the class $R_{0}$ of renewal sequences (cf. section 4.1) suggests the existence of relations between $F_{\infty}$ and the classes of $p$ functions and renewal densities, both of which can be considered as continuous analogues of $R_{0}$.

Let us first give the definition and some properties of p-functions; they can be found in Kingman (1972).

DEFINITION 5.5.1. A function $p$ on $(0, \infty)$ is said to be a p-function if there exists a $\{0,1\}$-valued stochastic process $\{z(t)\}_{t>0}$ (called a regenerative phenomenon) such that

$$
\text { (5.5.1) } \quad P\left(z\left(t_{1}\right)=\ldots=z\left(t_{n}\right)=1\right)=\prod_{k=1}^{n} p\left(t_{k}-t_{k-1}\right) \quad\left(0=t_{0}<t_{1}<\ldots<t_{n} ; n \in \mathbb{N}\right) .
$$

In a similar way one can define a discrete-time regenerative phenomenon $\{z(n)\}_{n \in \mathbb{N}_{0}}$. The sequence $\left\{u_{n}\right\}_{0}^{\infty}$ that then replaces the function $p$ in (5.5.1), can be shown to be a renewal sequence, and conversely. From (5.5.1) it follows that a p-function $p$ satisfies:

$$
(5.5 .2) \quad p(t)=p(z(t)=1) \quad(t>0),
$$

and hence

$$
(5.5 .3) \quad 0 \leq p(t) \leq 1 \quad(t>0) .
$$

p-functions satisfy many other inequalities; in fact, they can be characterized as follows (cf. the definition of renewal sequences). 
THEOREM 5.5.2. A function $p$ on $(0, \infty)$ is a p-function iff $p$ satisfies

$$
F\left(t_{1}, \ldots, t_{n} ; p\right) \geq 0, \sum_{k=1}^{n} F\left(t_{1}, \ldots, t_{k} ; p\right) \leq 1
$$

for all $n \in \mathbb{N}$ and $0<t_{1}<\ldots<t_{n}$, where

$$
\begin{aligned}
& F\left(t_{1}, \ldots, t_{n} ; p\right):=p\left(t_{n}\right)-\sum_{1 \leq i<n} p\left(t_{i}\right) p\left(t_{n}-t_{i}\right)+ \\
& +\sum_{1 \leq i<j<n} p\left(t_{i}\right) p\left(t_{j}-t_{i}\right) p\left(t_{n}-t_{j}\right) \cdots+(-1)^{n-1} p\left(t_{1}\right) \prod_{k=2}^{n} p\left(t_{k}-t_{k-1}\right) .
\end{aligned}
$$

COROLLARY 5.5 .3 . If $p$ is a p-function, then

$$
(5.5 .4) \quad p(s) p(t) \leq p(s+t) \leq p(s) p(t)+1-p(s) \quad(s, t>0) .
$$

The following two properties are of special interest.

THEOREM 5.5.4. If $p_{1}$ and $p_{2}$ are p-functions, then so is the function $p$, defined by

$$
p(t):=p_{1}(t) p_{2}(t) \quad(t>0)
$$

THEOREM 5.5.5. If $p$ is a p-function, if $h>0$, and if $u_{n}(h):=p(n h)(n \in \mathbb{N})$, $u_{0}(h):=1$, then

$$
\left\{u_{n}(h)\right\}_{0}^{\infty} \in R_{0} \text {. }
$$

in which case $\left\{u_{n}(h)\right\}$ is associated with $f_{n}(h):=F(h, 2 h, \ldots, n h ; p)$.

We shall connect $F_{\infty}$ with the class $P$ of standard p-functions, i.e. p-functions'p with the property

$$
\text { (5.5.5) } \lim _{t \downarrow 0} p(t)=1 \text {. }
$$

The class $R_{0}$ of renewal sequences coincides with the class of diagonal transition probabilities corresponding to any state in any (discrete-time) Markov chain (cf. theorem 4.1.6(i)). There exists only a partial analogue of this result for $P$.

THEOREM 5.5.6. If $\{x(t)\}_{t \geq 0}$ is a standard, continuous-time Markov chain on the countable state space $S$, if $a \in S$, and if 
(5.5.6) $\quad p(t):=P(x(t)=a \mid x(0)=a) \quad(t>0)$,

then $p \in P$.

The following theorem shows what functions can arise as LT'S of standard p-functions.

THEOREM 5.5.7. A function $\varphi$ on $(0, \infty)$ is the LT of a function $p \in P$ iff there exists a measure $\nu$ on $(0, \infty]$ satisfying

$(5.5 .7) \quad \int_{(0, \infty]}\left(1-e^{-x}\right) v(d x)<\infty$,

such that $\varphi$ has the form

$(5.5 .8) \quad \varphi(\tau)=\left\{\tau+\int_{(0, \infty]}\left(1-e^{-\tau x}\right) \nu(d x)\right\}^{-1} \quad(\tau>0)$

$(\nu$, which is unique, is called the canonical measure of $p \in P)$.

Before giving the relation between $F_{\infty}$ and $P$, we state some simple, but useful properties of $P$. Let $p \in P$ with canonical measure $V$. Then from the inequality $(5.5 .4)$ it can be shown (put $p(0)=1$ ) that $p$ is positive and uniformly continuous on $[0, \infty)$, and that $p(\infty):=\lim _{t \rightarrow \infty} p(t)$ exists in $[0,1]$, $-p_{+}^{\prime}(0):=\lim _{t \downarrow 0}\{1-p(t)\} / t$ in $[0, \infty]$ and $\ell:=\lim _{t \rightarrow \infty}\{-\log p(t)\} / t$ in $[0, \infty)$, while furthermore

$$
(5.5 .9) \quad p(\infty)=\left\{1+\int_{(0, \infty]} x v(d x)\right\}^{-1},-p_{+}^{\prime}(0)=v((0, \infty])
$$

and

$(5.5 .10) \quad \exp \left[p_{+}^{2}(0) t\right] \leq p(t) \leq \exp [-\ell t] \quad(t>0)$.

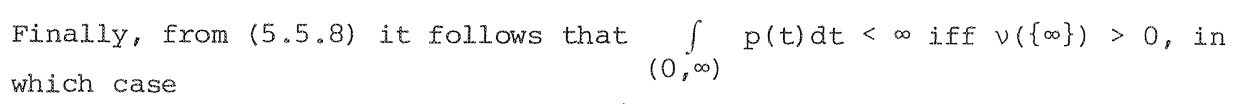

(5.5.11) $\int_{(0, \infty)} p(t) d t=v(\{\infty\})^{-1}$.

THEOREM 5.5.8.

(i) Let $F \in F_{\infty}$ wi.th $\ell(F)=0$, and let $\gamma>0$ in the canonical representation $(\gamma, N)$ of $F(c f .(5.4 .16))$. Then $F$ has a continuous density $f$, 
for which $f(0+)$ exists in $(0, \infty)$, and

$$
p(x):=f(x) / f(0+) \in P \text {. }
$$

(ii) Let $p \in P$ with canonical measure $\nu$, and let $\nu(\{\infty\})>0$. Then

$$
f(x):=v(\{\infty\}) p(x) \in F_{\infty}^{\prime} \text {. }
$$

(iii) Let $\mathrm{p} \in P$, and let $\mathrm{a}>0$. Then

$$
f(x):=e^{-a x} p(x) / \tilde{p}(a) \in F_{\infty}^{\prime}
$$

PROOF. One easily obtains parts (i) and (ii) by using theorem 5.4 .13 and (5.5.11), and by compaxing the canonical representations (5.4.16) for $F_{\infty}$ and $(5.5 .8)$ for $P$. Here the following relation holds between $(\gamma, N)$ and $\nu$ : $(5.5 .12) \quad \gamma \nu(d x)=a N(x)$ on $(0, \infty), \gamma v(\{\infty\})=1$

To prove part (iii), we define $\pi(x):=e^{-\operatorname{axp}}(x)(x \geq 0)$. Then in view of theorem 5.5 .4 we have $\pi \in P$. Since by $(5.5 .11)$ the canonical measure of $\pi$ has mass $\hat{\mathrm{P}}(\mathrm{a})^{-1}>0$ at $\infty$, we can apply part (ii), and (iii) follows.

By means of theorem 5.5 .8 it is possible to obtain properties of the class $\left\{F \in F_{\infty} \mid \ell(F)=0, \gamma>0\right\}$ from those of $P$ (and conversely), and one could try to extend these properties to the whole class $F_{\infty}^{\prime \prime}$ or, if the property considered can be transfered to df's, to $F_{\infty}$. However, we shall restrict ourselves to the following interesting analogue of theorem 5.5 .4 for $F_{\infty}$ (see also Hawkes (1977), who gives a similar result for potential kernels (cf. the end of the present section)).

THEOREM 5.5.9. Let $f$ be the continuous density of a df $F \in F_{\infty}$ with $l(F)=0$ and $\gamma>0(i . e . f(0+) \in(0, \infty))$ in its canonical representation $(\gamma, N)$ (cf. theorem 5.4.13), and let $G \in F_{\infty}$ with $\ell(G)=0$. Then there exists $c>0$ such that the function

$(5.5 .13) \quad H(x):=C \int_{[0, x]} f(y) d G(y), \quad(x \geq 0)$

is a de in $F_{\infty}$ with $\ell(H)=0$.

PROOF. First we note that by the boundedness of $f$ (cf. (5.4.30)) (5.5.13) defines a df $\mathrm{H}$ for a suitably chosen $\mathrm{c}>0$. By the remark, following lemma 5.4.18, there exist df's $G_{n}(n \in \mathbb{N})$ in $F_{\infty}$ with $\ell\left(G_{n}\right)=0$ and with $\gamma>0$ in 
their canonical representation such that $G$ is the weak limit of the sequence $\left\{G_{n}\right\}$. Since $f$ is continuous and bounded, it follows by the extended version of Helly's second theorem that

$$
(5.5 .14) \hat{H}(\tau)=\lim _{n \rightarrow \infty} c \int_{[0, \infty)} e^{-\tau x} f(x) d G_{n}(x) .
$$

By theorem 5.5.8(i), each of the $d f^{\prime} s G_{n}$ has a continuous density $g_{n}$, for which $g_{n}(x) / g_{n}(0+) \in P$. Applying theorem 5.5.4, we see that also $f(x) g_{n}(x) /\left\{f(0+) g_{n}(0+)\right\} \in P$, and hence (cf. theorem 5.5.8(ii)) $f(x) g_{n}(x)$ is, save for norming, a density in $F_{\infty}^{\prime}$. By $(5.5 .14)$ it now follows that $\mathrm{H}$ is the weak limit of a sequence of $\mathrm{df}^{\prime} \mathrm{s}$ in $F_{\infty}^{\prime}$, and hence (cf. theorem 5.3.1) $H \in F_{\infty}$.

REMARK 5.5.10. If $f$ is the density of a df $F \in F_{\infty}^{\prime \prime}$ for which $f(0+)=\infty$, then the integral in $(5.5 .13)$ is not necessarily convergent for every df $G \in F_{\infty}$ with $\ell(G)=0$ : take, for instance, a gamma df with second parameter $v=\frac{1}{2}$ for both $F$ and $G$.

The renewal sequences have a second continuous analogue, the renewal densities. This will be apparent from theorem 4.1 .5 and the following definition and properties of (pure) renewal functions (cf. Smith (1958)). Let $\mathrm{T}_{1}, \mathrm{~T}_{2}, \ldots$ be nonnegative, independent $r \mathrm{~V}^{\prime} \mathrm{S}$ wi.th common df $\mathrm{F}$. Then the sequence $\left\{s_{n}\right\}_{1}^{\infty}$, defined by

$$
S_{n}:=\sum_{k=1}^{n} T_{k} \quad(n \in \mathbb{N}),
$$

is called the renewal process associated with $F$. The renewal function $U$ associated with $F$, is defined by

$$
U(x):=E_{x^{\prime}} \text { with } N_{x}:=\#\left(\left\{n \in \mathbb{N} \mid S_{n} \leq x\right\}\right) \text {. }
$$

Both $\left\{\mathrm{S}_{n}\right\}$ and $U$ are called persistent if $F$ is proper, and transient if $F$ is defective. The renewal function $U$ is the solution of the renewal equation associated with $\mathrm{F}$ :

$(5.5 .15) \quad U(x)=F(x)+\int_{[0, x]} U(x-y) d F(y) \quad(x \in \mathbb{R})$,

and $U$ satisfies 
$(5.5 .16) \quad U(x)=\sum_{n=1}^{\infty} F^{* n}(x) \quad(x \in \mathbb{R})$

If $F$ has a density $f$, then $U$ has a density $u$, the renewal density, which satisfies

$(5.5 .17) u(x)=f(x)+\int_{[0, x]} u(x-y) f(y) d y \quad$ (almost all $\left.x \in I R\right)$.

First, let us consider a transient renewal function $U$. It is associated with $\mathrm{pF}$, for $\mathrm{p} \in(0,1)$ and $\mathrm{F}$ a proper df on $[0, \infty)$. By $(5.5 .15)$ it follows that the LST $\hat{U}$ of U satisfies

$(5.5 .18) \quad \hat{U}(\tau)=\frac{p \hat{F}(\tau)}{1-p \hat{F}(\tau)}$

from which, using corollary 5.4.30, we obtain the following relation with $F_{\infty}$

THEOREM 5.5.11. Let $\mathrm{U}$ be a transient renewal function, associated with pF $(0<\mathrm{p}<1)$. Then:

(i) $\hat{H}(\tau):=(1-p) p^{-1} \hat{U}(\tau)$ is a PLST in $F_{\infty}$ with $\ell(H)=0$ iff $F \in F_{\infty}$ with $\ell(F)=0$;

(ii) $(1-p)\{1+\hat{U}(\tau)\}$ is a PLST in $F_{\infty}^{+}$.

Next, let us consider the persistent case. Then we have the following relations for a renewal function associated with $\mathrm{F}$ :

$(5.5 .19) \quad \hat{U}(\tau)=\frac{\hat{F}(\tau)}{1-\hat{F}(\tau)}$ and $\hat{F}(\tau)=\frac{\hat{U}(\tau)}{1+\hat{U}(\tau)} \quad(\tau>0)$.

Daley (1965) characterizes the renewal functions $U$ for which $\mu U$ is again a renewal function for all $\mu>0$. His result is not only most easily formulated by means of the class $F_{\infty}$, but it also immediately follows from theorem 5.4.29; one need only observe that if $U$ is the renewal function associated with $F$ and if $\mu U$ is a renewal function, then (cf. (5.5.19)) $\mu U$ is associated with $\mathrm{F}_{\mu}$, given by

$$
\text { (5.5.20) } \hat{F}_{\mu}(\tau)=\frac{\mu}{\mu-1+\hat{E}(\tau)^{-1}} \quad(\tau \geq 0) .
$$

This proves Daley"s result, which we formulate as follows. 
THEOREM 5.5.12. Let $U$ be a (persistent) renewal function, associated with F. Then:

(i) $\mu \mathrm{U}$ is a renewal function for all $\mu \in(0,1]$;

(ii) $\mu U$ is a renewal function for all $\mu>0$ iff $F \in F_{\infty}$ with $\ell(F)=0$, in which case the af $F_{\mu}$ that $\mu U$ is associated with, is also in $F_{\infty}$ with $\ell\left(F_{\mu}\right)=0$.

Some authors (e.g. Feller (1971)) put $S_{0}=0$ and count this as a renewal epoch, i.e. they consider $1_{[0, \infty)}(x)+U(x)$ in stead of $U(x)$. In connection with this, Runnenburg (1965) notes that for most purposes it is sufficient to consider only renewal functions associated with a compound geometric df on $[0, \infty)$. In fact, he gives the following rather remarkable result (cf. theorem 5.5.11(ii)), which is easily verified.

LEMMA 5.5.13. If $U$ is the renewal function associated with the df $F$, then the function

$(5.5 .21) \quad V(x):=1_{[0, \infty)}(x)+U(x) \quad(x \in \mathbb{R})$

is a renewal function associated with a df $G \in F_{\infty}^{+}$, given by

$(5.5 .22) \quad \hat{G}(\tau)=\{2-\hat{\mathrm{F}}(\tau)\}^{-1} \quad(\tau \geq 0)$.

In view of theorem 5.5 .12 this lemma can be generalized as follows.

THEOREM 5.5.14. If $U$ is the renewal function associated with the of $F$, then for all $\mu>0$ the function

$$
V_{\mu}(x):=\mu\{1[0, \infty)(x)+U(x)\} \quad(x \in \mathbb{R})
$$

is a renewal function associated with a df in $F_{\infty}^{+}$.

If $F \in F_{\infty}$ has canonical representation $(\gamma, N)$, then by $(5.5 .19)$ the renewal function $U$, associated with $F$, satisfies

$$
(5.5 .23) \quad \hat{U}(\tau)=\left\{\gamma \tau+\int_{(0, \infty)}\left(1-e^{-\tau x}\right) d N(x)\right\}^{-1} \quad(\tau>0) .
$$

If $\gamma>0$, then by theorem 5.5 .7 and $(5.5 .11)$ it follows that $U$ has a continuous renewal density $u$, for which $p(x):=\gamma u(x)$ is a standard p-function with $\int_{(0, \infty)} p(x) d x=\infty$. Obviously, the converse is also true, i.e. (cf. theo- 
rem 5.5 .12 ) we have the following relation between renewal densities and p-functions (see also Daley (1965) and Kingman (1972)), which, by theorem 5.5 .8 (iii), also yields a relation between renewal densities and $F_{\infty}^{\prime}$

THEOREM 5.5.15. Let $u$ be a continuous function on $[0, \infty)$ with $u(0)=1$. Then $\mu u$ is a (persistent) renewal density for all $\mu>0$ iff $u \in P$ and $(0, \infty)$

$$
\int u(x) d x=\infty \text {. }
$$

Recently, Forst (1978) proved Daley's result by making use of the fact that a nondecreasing function $U$, vanishing on $(-\infty, 0)$, is a persistent renewal function iff the measure $\mu$, with df $1_{[0, \infty)}(x)+U(x)(x \in \mathbb{R})$, is a potential kernel on $[0, \infty)$ with infinite mass. Here, a potential kernel on $[0, \infty)$ is a measure $\mu$ of the form

$$
(5.5 .24) \mu=\int_{(0, \infty)} \mu_{t} d t
$$

where $\left(\mu_{t}\right)_{t>0}$ is a convolution semigroup of possibly defective probability measures on $[0, \infty)$ (see e.g. Berg \& Forst (1975)).

The potential kernels are intimately connected with the class $F_{\infty}$. This is apparent from the canonical representations. In terms of LST's, (5.5.24) can be rewritten as

$$
(5.5 .25) \hat{\mu}(\tau)=\int_{(0, \infty)}\{\hat{p} \hat{H}(\tau)\}^{t} d t=\{-\log p-\log \hat{H}(\tau)\}^{-1} .
$$

where $0<p \leq 1$ and $\mathrm{H} \in F_{0}$. Note that for $\mathrm{p}<1$ we have, save for norming, a special case of theorem 5.4.34: take for G in (5.4.53) an exponential df. Using for $\hat{H}$ the representation $(\gamma, N)$ from theorem 1.7.1, we obtain from (5.5.25) the canonical representation for the LST $\mu$ of a potential kernel $\mu$ :

$$
(5.5 .26) \hat{\mu}(\tau)=\left\{\beta+\gamma \tau+\int_{(0, \infty)}\left(1-e^{-\tau x}\right) d N(x)\right\}^{-1} \quad(\tau>0) .
$$

where $\beta \geq 0, \gamma \geq 0$ and $N$ is a nondecreasing function on $(0, \infty)$ with $N(\infty)=0$ and $\int_{(0,1)} x \mathrm{dN}(\mathrm{x})<\infty$ (not simultaneously $\beta=0, \gamma=0, N \equiv 0$ ). In fact, (5.5.26) reduces to the canonical representations $(5.4 .16)$ for $F_{\infty}$ and (5.5.8) for $P$, if we take $\beta=1$ and $\gamma=1$, respectively. Furthermore, if $U$ is the renewal function associated with $\mathrm{pF}$, where $0<\mathrm{p} \leq 1$ and $\mathrm{F}$ is a (proper) df on $[0, \infty)$, then, obviously, the LST 


$$
1+\hat{U}(\tau)=\{1-\hat{p} \hat{F}(\tau)\}^{-1} \quad(\tau>0)
$$

is of the form (5.5.26) with $\gamma=0$ and $N$ bounded. Thus, properties of $F_{\infty}$, $P$ and renewal functions can be obtained from known properties of potential kernels (cf. Berg \& Forst (1978)).

Finally, we note that the potential kernels correspond with more general renewal functions. The continuous-time analogue of the renewal process $\left\{s_{n}\right\}_{1}^{\infty}$ (cf. p. 181), regarded as a discrete-time process, is an increasing process $\left\{s_{t}\right\}_{t>0}$ that is inf div, i.e.

$$
\mathrm{Ee}^{-\tau S}=\{\hat{\mathrm{p}} \hat{\mathrm{F}}(\tau)\}^{t} \quad(\tau \geq 0),
$$

where $0<\mathrm{p} \leq 1$ and $\mathrm{F} \in F_{0}$. Now, the analogue of the renewal function $U$ is the function $V$, defined by ( $\lambda$ is the Lebesgue measure)

$$
V(x):=E \lambda\left(\left\{t>0 \mid S_{t} \leq x\right\}\right)=\int_{(0, \infty)} p^{t_{F}}{ }^{* t}(x) d t,
$$

which shows that the potential kernels, considered by e.g. Hawkes (1977). are identical to those defined in $(5.5 .24)$ or $(5.5 .25)$.

\subsection{Other classifications}

In section 2.5 we introduced the classes $C_{0}^{u}$ of compound negative--binomial lattice distributions with parametex $u>0$. They define a classification of $C_{1}$, and can be characterized as follows:

$$
\mathrm{P} \in \mathrm{C}_{\mathrm{O}}^{\mathrm{u}} \Leftrightarrow \mathrm{P}^{1 / \mathrm{u}} \text { is a pgf in } \mathrm{C}_{0} \text {. }
$$

Now, for the sake of completeness, we briefly consider the analogous classes $F_{\infty}^{u}$ of distributions on $[0, \infty)$.

DEFINITION 5.6.1. For $u>0$ a df $F$ on $[0, \infty)$ is said to be in the class $F_{\infty}^{u}$ iff $\hat{\mathrm{F}}^{1 / \mathrm{u}}$ is a PLST in $F_{\infty}$.

If $F$ is a df on $[0, \infty)$, if $\gamma>0$ and if $\hat{F}^{\gamma}$ is a PLST (of $F^{* \gamma}$ ), then the $\varphi_{0}-$ function of $F^{* \gamma}$ is equal to that of $F$, multiplied by $\gamma$. Hence, by lemma 5.1 .6 we have in this case

$(5.6 .1) \quad \ell\left(F^{* \gamma}\right)=\gamma \ell(F)$.

Now, by theorem 5.4 .5 it follows that a PLST $\hat{F}$ in $F_{\infty}^{u}$ with $l(F)=0$ has the 
canonical form

$$
\text { (5.6.2) } \quad \hat{\mathrm{F}}(\tau)=\{1-\log \hat{\mathrm{H}}(\tau)\}^{-\mathrm{U}} \quad(\tau \geq 0) .
$$

with $H \in F_{0}$. Thus, the df's $F$ in $F_{\infty}^{u}$ with $h(F)=0$ can be regarded as "Compound garma" df's (cf. remark 5.4.36).

Since $F_{\infty}^{+}$is the class of compound geometric distributions on $[0, \infty)$, the class $\left(F_{\infty}^{\mathrm{u}}\right)^{+}$of df'S $\mathrm{F}$ in $F_{\infty}^{\mathrm{u}}$ with $\mathrm{F}(0)>0$ coincides with the class of compound negative-binomial distributions on $[0, \infty)$ with parameter $u$ (cf. theorem 1.6.6). Also, $F_{\infty}^{+}$is dense in $\left\{F \in F_{\infty} \mid \ell(F)=0\right\}$ in the sense of weak convergence (cf. (5.4.1)), and this result, together with (5.6.1), immediately yields the following characterization of $F_{\infty}^{u}$ (cf. theorem 1.4.15).

THEOREM 5.6.2. For $u>0$ a af $F$, with $\ell(F)=0$, is in $F_{\infty}^{u}$ iff $F$ is the weak limit of compound negative-binomial df's with parameter $u$, i.e. iff $\hat{F}$ has the form

$$
\text { (5.6.3) } \hat{\mathrm{F}}(\tau)=\lim _{n \rightarrow \infty}\left\{\frac{1-p_{n}}{1-p_{n} \hat{G}_{n}(\tau)}\right\}^{u} \quad(\tau \geq 0),
$$

where $0<p_{n}<1$ and $G_{n}$ is a df on $[0, \infty)(n \in \mathbb{N})$.

The family $\left(F_{\infty}^{\mathrm{u}} \mid u>0\right.$ ) defines a classification of $F_{0}$ : similar to the proof of theorem 2.5 .3 it can be shown that

$(5.6 .4) \quad \mathrm{u}<\mathrm{v} \Rightarrow F_{\infty}^{\mathrm{u}} \subset F_{\infty}^{\mathrm{V}}$

Furthermore, $\underset{u>0}{U} F_{\infty}^{u}$ is dense in $F_{\circ}$; in fact, if $F \in F_{0^{\prime}}$ then $F$ is the weak limit of the sequence of df's $F_{n}$, defined by

$$
(5.6 .5) \quad \hat{F}_{n}(\tau):=\left\{\frac{n}{n-\log \hat{F}(\tau)}\right\}^{n} \quad(\tau \geq 0)
$$

which $(c f .(5.6 .2))$ is a PLST in $F_{\infty}^{n}(n \in \mathbb{N})$.

Another interpolation between $F_{\infty}$ and $F_{0}$ is suggested by the relation $(5.4 .2)$ between the $\psi_{\infty}$-function and the $\varphi_{0}$-function of a df $F$ on $[0, \infty)$ : we briefly indicate the analogues to the classes $H_{\gamma}(\gamma \geq 0)$ from definition 2.5.7.

DEFINITION 5.6.3. For $\gamma \geq 0$ a df $F$ on $[0, \infty)$ is said to be in the class $L_{\gamma}$ if the function 
$(5.6 .6) \quad-\hat{F}^{\prime}(\tau) / \hat{F}(\tau)^{1+\gamma} \quad(\tau>0)$

is comp mon.

obviously, $L_{0}=F_{0}$ and $L_{1}=\left\{F \in F_{\infty} \mid \ell(F)=0\right\}$ (cf. theorem 5.4.2). Now, let $\gamma>0$ and let $F \in L_{\gamma}$. Then the function in (5.6.6) has a finite limit $c$, say, as $\tau \rightarrow \infty$, and therefore by lemma 5.1 .6 it follows that

$$
\ell(F)=\lim _{\tau \rightarrow \infty}-\hat{F}^{\prime}(\tau) / \hat{F}(\tau)=\mathrm{CF}(0)^{\gamma},
$$

and hence

$$
\text { (5.6.7) } \quad F \in L_{\gamma} \Rightarrow \ell(F)=0 \quad(\gamma>0) .
$$

Fuxthermore, if $\mathrm{F} \in L_{\gamma}$, then $\hat{\mathrm{F}}^{-\gamma}$ has a comp mon derivative; hence by lemma $1.3 .8(v i) \hat{F}^{\gamma}=\left\{\hat{F}^{-\gamma}\right\}^{-1}$ is a PLST, and $i$ ts $\psi_{\infty}$-function is comp mon. Now it easily follows that

(5.6.8) $\quad F \in L_{\gamma} \Leftrightarrow \hat{F}^{\gamma}$ is a PLST in $L_{1}$,

which by $(5.6 .7)$ can be reformulated as follows.

THEOREM 5,6,4. For $\gamma>0$ the following relation holds:

$$
L_{\gamma}=\left\{F \in F_{\infty}^{1 / \gamma} \mid \ell(F)=0\right\}
$$

Thus, for df's $F$ on $[0, \infty)$ with $\ell(F)=0$ the family $\left(L_{\gamma} \mid \gamma>0\right)$ defines the same classification of $F_{0}$ as $\left(F_{\infty}^{u} \mid u>0\right)$.

\subsection{Further generalizations}

In analogy to the investigations in chapter 3 we could consider tot dec, tot fact and tot-dec(1) PLST's. We only mention the following analogue of theorem 3.2.4, and note that the analogue of theorem 3.4 .5 has already been given in theorem 5.4.29(iii).

THEOREM 5.7.1. A df $\mathrm{F}$ on $[0, \infty)$ is inf div iff for all $\lambda>0$, or, equivalent$1 y$, for all $\lambda \in(0, \varepsilon)$ (some $\varepsilon>0$ ), there exists a df $F_{\lambda}$ on $[0, \infty$ ) such that $\hat{\mathrm{F}}$ can be written as

$$
\hat{F}(\tau)=\frac{\hat{F}(\tau+\lambda)}{\hat{F}(\lambda)} \hat{F}_{\lambda}(\tau) \quad(\tau \geq 0) .
$$


Inchapter 4 we studied extensions of the $C_{\alpha}$ 's, the classes $R_{\alpha}$ of generalized renewal sequences. Similarly, one could consider the functional equations (5.1.7) for nondecreasing functions $F$ that are not necessarily df's. This would give rise to classes of generalized p-functions, renewal functions and potential kernels.

In view of a further generalization one might ask to what extent the results of this monograph can be extended to distributions on the whole real line. Trying to extend the classification of $F_{0}$ by the $F_{\lambda}$ 's to df's on $\mathbb{R}$, one encounters the difficulty that the PLST $\hat{F}(\tau+\lambda) / \hat{F}(\lambda)$, which plays an essential role for $F_{\lambda}$, has no obvious analogue for general df's; $\widetilde{F}(t+\lambda) / \widetilde{F}(\lambda)$ is, in general, not a chf for $\lambda>0$. Also, no functional equations are known that characterize the class of all inf div df's on $\mathbb{R}$.

Another possibility is writing an inf div chf $\tilde{F}$ as a function of chf's of df's on $[0, \infty)$, and then requiring that these $d f^{\prime} s$ are in $F_{\lambda}$ of course, every chf $\widetilde{\mathrm{F}}$ can be written as

(5.7.1) $\widetilde{\mathrm{F}}(t)=(1-\mathrm{p}) \widetilde{\mathrm{F}}_{1}(t)+\mathrm{p} \widetilde{\mathrm{F}}_{2}(-t) \quad(t \in \mathbb{R})$,

where $0 \leq p \leq 1$, and $F_{1}$ and $F_{2}$ are $d f^{\prime} s$ on $[0, \infty)$. However, if $F_{1}$ and $F_{2}$ are in $F_{0}$, then $F$ is not necessarily inf div.

The following decomposition gives a better result.

DEFINITION 5.7.2. For $0 \leq \lambda \leq \infty$ a df $F$ on $\mathbb{R}$ is said to be in the class $G_{\lambda}$ if there exist df's $F_{1}$ and $F_{2}$ in $F_{\lambda}$ such that

$$
\text { (5.7.2) } \quad \widetilde{F}^{\prime}(t)=\widetilde{F}_{1}(t) \widetilde{F}_{2}(-t) \quad(t \in \mathbb{R}) .
$$

Obviously, we have the desired monotonicity property:

$$
G_{\lambda} \subset G_{\mu} \text { if } 0 \leq \mu \leq \lambda \leq \infty \text {, }
$$

but $G_{0}$ does not coincide with the class of all inf div df's on $\mathbb{R}$; in fact, by theorem 1.7.11 it is seen that an'inf div df $F$ is in $G_{0}$ iff its Lévy representation $\left(a, \sigma^{2}, M\right)$ satisfies

$$
(5.7 .3) \quad \sigma^{2}=0, \int_{(-1,1) \backslash\{0\}}|x| d M(x)<\infty .
$$

Therefore, for $0 \leq \lambda \leq \infty$ we introduce the classes $G_{\lambda}$ of $d f^{\prime}$ 's that are weak limits of $d f^{\prime} s$ in $G_{\lambda}$. Now, observing that the compound Poisson df's on IR 
are in $G_{0}$ (cf. theorem 1.7 .13 and $(5.7 .3)$ ), we conclude from De Finetti's theorem (theorem 1.4 .15 ) that $\vec{G}_{0}$ is the class of $\alpha$ ll inf div df's on $\mathbb{R}$. As obviously

$$
\bar{G}_{\lambda} \subset G_{\mu} \text { if } 0 \leq \mu \leq \lambda \leq \infty 。
$$

the family $\left(\vec{G}_{\lambda} \mid 0 \leq \lambda \leq \infty\right)$ defines a classification of the inf div df's on IR. Unfortunately, this does not lead to a characterization of $\bar{G}_{\lambda}$ by a single functional equation.

We note that the classification of $F_{0}$ given by $\left(F_{\infty}^{u} \mid u>0\right.$ ) (cf. section 6 ), is easily extended to general df's on $\mathbb{R}$ : consider chf's $\tilde{F}$ of the form

$$
\widetilde{F}(t)=\{1-\log \tilde{H}(t)\}^{-u} \quad(t \in \mathbb{R}),
$$

with $\mathrm{H}$ an inf div df on $\mathbb{R}$.

Finally, we consider the multivariate case. Generalizing the functional equations by means of which Horn \& steutel (1978) characterize the distributions of nonnegative multivariate inf div random vectors, we can obtain a classification of these distributions analogous to that of $F_{0}$ by the $F_{\lambda_{2}}$ s. we illustrate this by giving this characterization for distributions on $\mathbb{N}_{0}^{2}$.

THEOREM 5.7.3. Let $\left\{\mathrm{p}_{\mathrm{k}, l}\right\}$ be a probability distribution on $\mathbb{N}_{0}^{2}$ with $\mathrm{p}_{0,0}>0$. Then there exists a unique solution $\left\{a_{k}, l\right\}$ with $a_{0,0}=0$ of the following system of recurrence relations:

$$
\begin{aligned}
& (k+1) p_{k+1, \ell}=\sum_{i=0}^{k} \sum_{j=0}^{\ell}(i+1) a_{i+1, j} p_{k-i, \ell-j} \quad\left(k, \ell \in \mathbb{N}_{0}\right) . \\
& (\ell+1) p_{k, \ell+1}=\sum_{i=0}^{k} \sum_{j=0}^{\ell}(j+1) a_{i, j+1} p_{k-i, l-j}
\end{aligned}
$$

Furthermore, $\left\{p_{k, l}\right\}$ is inf div iff $a_{k, l} \geq 0$ for all $k, l \in \mathbb{N}$, in which case

$$
\sum_{k, l} a_{k, l}=-\log p_{0,0}<\infty
$$

Now, we can replace the factors $k+1$ and $\ell+1$ in $(5.7 .4)$ by

or by

$$
\begin{aligned}
& \left(1-\alpha^{k+1}\right) /(1-\alpha) \text { and }\left(1-\alpha^{\ell+1}\right) /(1-\alpha), \\
& \left(1-\alpha^{k+1}\right) /(1-\alpha) \text { and }\left(1-\beta^{\ell+1}\right) /(1-\beta),
\end{aligned}
$$

respectively, to obtain classifications of the inf div distributions on $\mathbb{N}_{0}^{2}$. 


\section{REFERENCES}

Ahmad, R. and Abouammoh, A.M. (1977). On the structure and applications of infinite divisibility, stability and symmetry in stochastic inference, Recent developments in statistics, J.R. Barra et al. (edi-tors), North-Holland Publishing Company, Amsterdam, etc.

Baxter, G. and Shapiro, J.M. (1960). On bounded infinitely divisible random variables, Sankhya $22,253-260$.

Berg, C. and Forst, G. (1975). Potential theory on locally compact abelian groups, Exg. der Math. Bd. 87, Springer, Berlin, etc.

Berg, C. and Forst, G. (1978). Infinitely divisible probability measures and potential kernels, to be published in: Lecture Notes in Math., springer, Berlin, etc.

Blum, J.R. and Rosenblatt, M. (1959). On the structure of infinitely divisible distributions, Pacific J. Math. 9, 1-7.

Bruijn, N.G. de and Erdös, P. (1951). Some linear and some quadratic recursion formulas I, Indag. Math. 13(5), 374-382.

Daley, D.J. (1965). On a class of renewal functions, Proc. Cam. Phil. Soc. 61, 519-526.

Fellex, W. (1968). An introduction to probability theory and its applications, vol. 1, 3-d ed., Wiley, New York.

Fellex, W. (1971). An introduction to probability theory and its applications, vol. 2, 2-nd ed., Wiley, New York.

Fisz, M. (1962). Infinitely divisible distributions: Recent results and applications, Ann. Math. Statist. 33, 68-84.

Fisz, M. and Varadarajan, V.S. (1963). A condition for absolute continuity of infinitely divisible distribution functions, $\mathrm{z}$. Wahrscheinlichkeitstheorie verw. Gebiete 1, 335-339.

Forst, G. (1978). Multiples of renewal functions; remark on a result of D.J. Daley, to be published in: Lecture Notes in Math. "Springer, Berlin, etc.

Gnedenko, B.V. and Kolmogorov, A.N. (1949, English ed. 1954). Limit distributions for sums of independent random variables, Addison-Wesley, Cambridge, Mass. 
Goldie, C.M. (1967). A class of infinitely divisible distributions, Proc. Cambridge Phil. Soc. 63, 1141-1143.

Grosswald, E. (1976). The student t-distribution of any degree of freedom is infinitely divisible, $z$. Wahrscheinlichkeitstheorie verw. Ge-biete $36,103-109$.

Gurland, J. (1957). Some interrelations among compound and generalized distributions, Biometrika 44, 265-268.

Harn, K. Van (1977). Opgave 43, Statistica Neerlandica (problem section) 31(4), 188-189.

Harn, K. van and Steutel, F.W. (1977). Generalized renewal sequences and infinitely divisible lattice distributions, stochastic Processes App 1. 5, 47-55.

Hawkes, J. (1977). Intersections of Markov random sets, $z$. Wahrscheinlichkeitstheorie verw. Gebiete 37, 243--251.

Hawkes, J. and Jenkins, J.D. (1978). Infinitely divisible sequences, Scand. Actuarial J. 2, 65-76.

Horn, R.A. and Steutel, F.W. (1978). On multivariate infinitely divisible distributions, Stochastic Processes Appl. 6, 139-151.

Johnson, N.L. and Kotz, S. (1969). Distributions in statistics: discrete distributions, Houghton Mifflin Company, Boston.

Kaluza, T. (1928). Uber die Koeffizienten reziproker Potenzreihen, Math. Zeitschrift 28, 161-170.

Katti, S.K. (1967). Infinite divisibility of integer-valued random varia-bles, Ann. Math. Statist. 38, 1306-1308.

Katti, S.K. (1977). Infinite divisibility of discrete distributions III, to be published.

Kelker, D. (1972). Infinite divisibility and variance mixtures of the normal distribution, Ann. Math. Statist. 42, 802-808.

Kendall, D.G. (1967). Renewal sequences and their arithmetic, Symposium on Probability Methods in Analysis, Lecture Notes in Mathematics 31, Springer, Berlin, etc.

Kingman, J.F.C. (1972). Regenerative phenomena, Wiley, London. 
Lamperti, J. (1958). On the coefficients of reciprocal power series, Ameri-can Math. Monthly $65,90-94$.

Lévy, P. (1937). Théorie de 1'addition des variables aléatoires, Gauthiervillars, Paris.

Linnik, Y.V. (1964). Decompositions of probability distributions, oliver and Boyd, London.

Lukacs, E. (1970). Characteristic functions, 2-nd ed., Griffin, London.

Miller, D. (1967). A note on passage times and infinitely divisible distributions, J. Appl. Probability 4 , 402-405.

Petrov, V.V. (1972, English ed. 1975). Sums of independent random variables, Springer, Berlin, etc.

Pólya, G. and Szegö, G. (1970). Aufgaben und Lehrsätze aus dex Analysis, Bd. 1, 4-th ed., Springer, Berlin, etc.

Runnenburg, J. Th. (1965). Discussion on Mr. Kingman"s paper, W.L. Smith and W.E. Wilkinson (editors), Proceedings of the Symposium on Congestion Theory, The University of North Caroline Press, Chapel Hi.11.

Smith, W.L. (1958). Renewal theory and its ramifications, J.R. Statist. Soc. B $20,243-302$.

Steute1, F.W. (1970). Preservation of infinite divisibility under mixing, and related topics, Math. Centre Tracts 33, Math. Centre, Amsterdam.

Steutel, F.W. (1973). Some recent results in infinite divisibility, stochastic Processes Appl. 1, 125-143.

Steutel, F.W. and Harn, K. Van (1978). Discrete analogues of self-decomposability and stability, to be published in: Ann. Probability.

Steutel, F.W. and Wolfe, S.J. (1977). On the asymptotic behaviour of moments of infinitely divisible distributions, Memorandum COSOR 77-06, Department of mathematics, Eindhoven University of Technology, Eindhoven, The Netherlands.

Thorin, $0 .(1977)$. On the infinite divisibility of the lognormal distribution. Scand. Actuarial J., 121-148. 
Tucker, H.G. (1961). Best one-sided bounds of infinitely divisible random variables, Sankhya Ser. A $24,387-396$.

Tucker, H.G. (1962). Absolute continuity of infinitely divisible distributions, Pacific J. Math. 12, 1125-1129.

Tucker, H.G. (1965). On a necessary and sufficient condition that an infinitely divisible distribution be absolutely continuous, Trans. Amex. Math. Soc. 118, 316-330.

vervaat, w. (1978). On a stochastic difference equation and a representation of nonnegative infinitely divisible random variables, to be published in: Adv. Appl. Prob.

Warde, W.D. and Katti, S.K. (1971). Infinite divisibility of discrete distributions II, Ann. Math. Statist. 42, 1088-1090.

widder, D.V. (1946). The Laplace Transform, Princeton University Press, Princeton.

Wolfe, S.J. (1971a). On the unimodality of I functions, Ann. Math. Statist. $42,912-918$.

Wolfe, S.J. (1971b). On moments of infinitely divisible distribution functions, Ann. Math. Statist. 42, 2036-2043.

Wright, E.M. (1967). A relationship between two sequences, Proc. London Math. Soc. 17, 296-304.

Yamazato, M. (1978). Unimodality of infinitely divisible distribution functions of class L, Ann. Probability 6, 523-531. 


\section{OTHER TITLES IN THE SERIES MATHEMATICAL CENTRE TRACTS}

A leaflet containing an order-form and abstracts of all publications mentioned below is available at the Mathematisch Centrum. Tweede Boerhaavestraat 49, Amsterdam-1005, The Netherlands. Oxders should be sent to the same address.

MCT 1 T. VAN DER WALT, Fixed and almost fixed points, 1963. ISBN 906196 0029.

MCT 2 A.R. BLOEMENA, Sampling from a graph, 1964. ISBN 9061960037.

MCT 3 G. DE LEVE, Generalized Markovian decision processes, part I: Model and method, 1964. ISBN 9061960045.

MCT 4 G. DE LEVE, Generalized Markovian decision processes, part II: Probabilistic background, 1964. ISBN 9061960053.

MCT 5 G. DE LEVE, H.C. TIJMS \& P.J. WEEDA, GeneraZized Markovian decision processes, Applications, 1970. ISBN 9061960517.

MCT 6 M.A. MAURICE, Compact ordered spaces, 1964. ISBN 9061960061.

MCT 7 W.R. VAN ZWET, Convex transformations of random variables, 1964. ISBN $906196007 \mathrm{x}$.

MCT 8 J.A. ZONNEVELD, Automatie numerical integration, 1964. ISBN 906196 0088.

MCT 9 P.C. BAAYEN, Universal morphisms, 1964. ISBN 9061960096.

MCT 10 E.M. DE JAGER, Applications of distributions in mathematical physics. 1964. ISBN $906196010 \mathrm{x}$.

MCT 11 A.B. PAALMAN-DE MIRANDA, Topological semigroups, 1964. ISBN 906196 0118.

MCT 12 J.A.TH.M. VAN BERCKEL, H. BRANDT CORSTIUS, R.J. MOKKEN \& A. VAN WIJNGAARDEN, Formal properties of newspaper Dutch, 1965. ISBN 9061960134.

MCT 13 H.A. LAUWERIER, Asymptotic expansions, 1966, out of print; replaced by MCT 54 and 67.

MCT 14 H.A. IAUWERIER, CaZculus of variations in mathematical physics, 1966. ISBN 9061960207.

MCT 15 2. DOORNBOS, SZippage tests, 1966. ISBN 9061960215.

MCT 16 J.W. DE BAKKER, Formal definition of programming Zanguages with an application to the definition of ALGOL 60, 1967. ISBN 906196 0223.

MCT 17 R.P. VAN DE RIET, EOrmuZa manipulation in ALGOL 60, part 1, 1968. ISBN 9061960258.

MCT 18 R.P. VAN DE RIET, Formila manipulation in ALGOL 60, part 2, 1968. ISBN $906196038 \mathrm{x}$.

MCT 19 J. VAN DER SLOT, Some propexties related to compactness, 1968. ISBN 9061960266 .

MCT 20 P.J. VAN DER HOUWEN, Finite difference methods for solving partial differential equations, 1968. ISBN 9061960274. 
MCT 21 E. WATTEL, The compactness operator in set theory and topology, 1968. ISBN 9061960282.

MCT 22 T.J. DEKKER, ALGOr 60 procedures in numerical algebra, part 1, 1968. ISBN 9061960290 .

MCT 23 T.J. DEKKER \& W. HOFFMANN, ALGOL 60 procedures in numerical algebra, part 2, 1968. ISBN 9061960304.

MCT 24 J.W. DE BAKKER, Recursive procedures, 1971. ISBN 9061960606.

MCT 25 E.R. PAERL, Representations of the Lorentz group and projective geometry, 1969. ISBN 9061960398.

MCT 26 EUROPEAN MEETING 1968, selected statistical papers, part I, 1968. ISBN 9061960312

MCT 27 EUROPEAN MEETING 1968, Selected statistical papers, part II, 1969. ISBN 9061960401 .

MCT $28 \mathrm{~J}$. OOSTERHOFF, Combination of one-sided statistical tests, 1969. ISBN $906196041 \mathrm{x}$.

MCT 29 J. VERHOEFF, Error detecting decimal codes, 1969. ISBN 9061960428.

MCT 30 H. BRANDT CORSTTUS, Excercises in computational linguistics, 1970. ISBN 9061960525 .

MCT 31 W. MOLENAAR, Approximations to the Poisson, binomial and hypergeom metric distribution functions, 1970. ISBN 9061960533.

MCT 32 L. DE HAAN, On regular variation and its application to the weak convergence of sample extremes, 1970. ISBN 9061960541.

MCT 33 F.W. STEUTEL, Preservation of infinite divisibitity under mixing and related topies, 1970. ISBN 9061960614.

MCT 34 I. JUHÄSZ, A. VERBEEK \& N.S. KROONENBERG, Cardinal functions in topology, 1971. ISBN 9061960622.

MCT 35 M.H. VAN EMDEN, An analysis of complexity, 1971. ISBN 9061960630.

MCT 36 J. GRASMAN, On the birth of boundary Zayers, 1971. ISBN 9061960649.

MCT 37 J.W. DE BAKKER, G.A. BLAAUW, A.J.W. DUIJVESTTJN, E.W. DIJKSTRA, P.J. VAN DER HOUWEN, G.A.N. KAMSTEEG-KEMPER, F.E.J. KRUSEMAN ARETZ, W.L. VAN DER FOEL, J.P. SCHAAP-KRUSEMAN, M.V. WILKES \& G. ZOUTENDIJK, MC-25 Informatica Symposium, 1971. ISBN 9061960657 .

MCT 38 W.A. VERLOREN VAN THEMAAT, Automatic analysis of Dutch compound words, 1971. ISBN 9061960738.

MCT 39 H. BAVINCK, Jacobi semies and approximation, 1972. ISBN 9061960746.

MCT 40 H.C. TIJMS, Analysis of $(s, S)$ inventory modets, 1972. ISBN 3061960754.

MCT 41 A. VERBEEK, Superextensions of topological spaces, 1972. ISBN 90 61960762 .

MCT 42 W. VERVAAT, Success epochs in Bernoulzi trials (with applications in number theory), 1972. ISBN 9061960770.

MCT 43 F.H. RUYMGAART, Asymptotic theory of rank tests for independence, 1973. ISBN 9061960819.

MCT 44 H. BART, Meromorphie operator valued functions, 1973. ISBN 9061960827. 
MCT 45 A.A. BALKEMA, Monotone transformations and limit Zaws, 1973. ISBN 9061960835 .

MCT 46 R.P. VAN DE RIET, ABC ALGOI, A portable Zanguage for formula manipuZation systems, part 1: The Zanguage, 1973. ISBN 9061960843.

MCT 47 R.P. VAN DE RIET, ABC ALGOL, A portable Zanguage for formiza manipuZation systems, part 2: The compizer, 1973. ISBN 9061960851.

MCT 48 F.E.J. KRUSEMAN ARETZ, P.J.W. TEN HAGEN \& H.L. OUdSHOORN, An ALGOL 60 compizer in ALGOL 60. Text of the MC-compizer for the $E L-X 8,1973$. ISBN $906196086 \mathrm{x}$.

MCT 49 H. KOK, Connected orderable spaces, 1974. ISBN 9061960886.

MCT 50 A. VAN WIJNGAARDEN, B.J. MAILLOUX, J.E.L. PECK, C.H.A. KOSTER, M. STNTZOFF, C.H. LINDSEY, L.G.L.T. MEERTENS \& R.G. FISKER (Eds), Revised report on the algowithmic Zanguage Al,GOI 68, 1976. ISBN 906196.0894.

MCT 51 A. HORDIJK, Dynamic programming and Markov potential theory. 1974. ISBN 9061960959.

MCT 52 P.C. BAAYEN (ed.), Topological structures, 1974. ISBN 9061960967.

MCT 53 M.J. FABER, Metrizability in generalized ordered spaces, 1974. ISBN 9061960975.

MCT 54 H.A. LAUWERIER, Asymptotic analysis, part 1, 1974. ISBN 9061960983.

MCT 55 M. HALI JR. \& J.H. VAN LINT (Eds), Combinatorics, part 1: Theory of designs, finite geometry and coding theory, 1974. ISBN 9061960991.

MCT 56 M. HALL JR. \& J.H. VAN LINT (Eds), Combinatorics, part 2: graph theory, foundations, partitions and combinatorial geometry, 1974. ISBN 9061961009

MCT 57 M. HALL JR. \& J.H. VAN LINT (Eds), Combinatorics, part 3: Combinatorial group theory, 1974. ISBN 9061961017.

MCT 58 W. ALBERS, Asymptotic expaniions and the deficiency concept in statistics, 1975. ISBN 9061961025 .

MCT 59 J.L. MIJNHEER, Sample path properties of stabie processes, 1975. ISBN 9061961076.

MCT 60 F. GÖBEL, Queueing models irvolving buffers, 1975. ISBN 9061961084.

* MCT $61^{\circ}$ P. VAN EMDE BOAS, Abstract resource-bound classes, part 1. ISBN 9061961092 .

* MCT 62 P. VAN EMDE BOAS, Abstract resource-bound classes, part 2. ISBN 9061961106.

MCT 63 J.W. DE BAKKER (ed.), Foundations of computer science, 1975. ISBN 9061961114.

MCT 64 W.J. DE SCHIPPER, Symmetric closed categories, 1975. ISBN 906195 1122

MCT 65 J. DE VRIES, Topological transformation groups 1 A categorical approach, 1975. ISBN 9061961130.

MCT 66 H.G.J. PIJLs, Locally convex algebras in spectral theory and eigenfunction expansions, 1976. ISBN 9061961149. 
* MCT 67 H.A. LAUWERIER, Asymptotic analysis, part 2. ISBN $906196119 \mathrm{x}$

MCT 68 P.P.N. DE GROEN, Singularly perturbed differential operators of second order, 1976. ISBN 9061961203.

MCT 69 J.K. LENSTRA, Sequencing by enumerative methods, 1977. ISBN 9061961254 .

MCT 70 W.P. DE ROEVER JR., Recursive program schemes: semantics and proof theory, 1976. ISBN 9061961270 .

MCT 71 J.A.E.E. VAN NUNEN, Contracting Markov decision processes, 1976. ISBN 9061961297.

MCT 72 J.K.M. JANSEN, Simple periodic and nonperiodic Lamé functions and theirs applications in the theory of conical waveguides,1977. ISBN 9061961300 .

* MCT 73 D.M.R. LEIVANT, Absoluteness of intuitionistic Zogic. ISBN $906196122 \%$.

MCT 74 H.J.J. TE RTELE, A theoretical and computational study of generalized aliquot sequences, 1976. IsBN 9061961319.

MCT 75 A.E. BROUWER, Treelike spaces and related connected topological spaces, 1977. ISBN 9061961327.

MCT 76 M. REM, Associons and the closure statement, 1976. ISBN 9061961351.

MCT 77 W.C.M. KALLENBERG. Asymptotic optimality of likelihood ratio tests in exponential families, 1977 ISBN 9061961343.

MCT 78 E. DE JONGE, A.C.M. VAN ROOJJ, Introduction to Riesz spaces, 1977. ISBN 9061961335.

MCT 79 M.C.A. VAN ZUIJLEN, Empirical distributions and rankstatistics, 1977. ISBN 9061961459.

MCT 80 P.W. HEMKER, A numerical study of stiff two-point boundary problems, 1977. ISBN 9061961467 .

MCT 81 K.R. APT \& J.W. DE BAKKER (eds), Foundations of computer science II, part I, 1976. ISBN 9061961408.

MCT 82 K.R. APT \& J.W. DE BAKKER (eds), Foundations of computer science II. part II, 1976. ISBN 9061961416.

* MCT 83 L.S. VAN BENTEM JUTTING, Checking Landau's "Grundiagen" in the AUTOMATH system, ISBN 9061961475.

MCT 84 H.L.I. BUSARD, The translation of the elements of Euclid from the Arabic into Latin by Hermann of Carinthia (?) books viz-xii, 1977. ISBN 9061961483.

MCT 85 J. VAN MILI, Supercompactness and WaZZman spaces, 1977. ISBN 9061961513.

MCT 86 S.G. VAN DER MEULEN \& M. VELDHORST, TOrYix I, 1978. ISBN 9061961521.

* MCT 87 S.G. VAN DER MEULEN \& M. VELDHORST, TOMmix II. ISBN $906196153 \mathrm{x}$

MCT 88 A. SCHRIJVER, Matroids and linking systems, 1977. ISBN 9061961548. 
MCT 89 J.W. DE ROEVER, Complex Fourier transformation and analytic functionals with unbounded carmers, 1978. ISBN 9061961556.

* MCT 90 L.P.J. GROENEWEGEN, Charactemization of optimal strategies in dynamic games. . ISBN 9061961564.

* MCT 91 J.M. GEYSEL. Transcendence in fields of positive characteristic, - ISBN 9061961572 .

* MCT 92 P.J. WEEDA, Finite generalized Markov programing, . ISBN 9061.961580 .

MCT 93 H.C. TIJMS (ed.) \& J. WESSELS (ed.), Markov decision theory, 1977. ISBN 9061961602 .

MCT 94 A. BIJLSMA, Simultaneous approximations in transcendental number theory, 1978. ISBN 90.61961629.

MCT 95 K.M. VAN HEE, Bayesian control of Markov chains, 1978. ISBN 9061961637.

* MCT 96 P.M.B. VITÁNYI, Lindenmayer systems: stmucture, languages, and growth functions, 1978 . ISBN 9061961645.

* MCT 97 A. FEDERGRUEN, Markovian control problems; functional equations and algorithms, 1978. ISBN 9061961653.

MCT 98 R. GEEL, Singular perturbations of hyperbolic type, 1978. ISBN 9061961661

MCT 99 J.K. LENSTRA, A.H.G. RINNOOY KAN \& P. VAN EMDE BOAS, Interfaces between computer science and operations research. 1978. ISBN $906196170 \mathrm{X}$.

* MCT 100 P.C. BAAYEN, D. VAN DULST \& J. OOSTERHOFF (Eds), Proceedings bicentennial congress of the Wiskundig Genootschap, part 1, ISBN 9061961688.

* MCT 101 P.C. BAAYEN, D. VAN DULST \& J. OOSTERHOFF (Eds), Proceedings bicentennial congress of the Wiskundig Genootschap, part 2, ISBN 9091961696.

MCT 102 D. VAN DULST, Reflexive and superreflexive Banach spaces, 1978. ISBN 9061961718

MCT $103 \mathrm{~K}$. VAN HARN, Classifying infinitely divisible distributions by functional equations, 1978 . ISBN 9061961726.

* MCT 104 J.M. VAN WOUWE, Go-spaces and generalizations of metrizability, - ISBN 9061961734 .

* MCT 105 R. HELMERS, Edgeworth expansions for linear combinations of order statistics, . ISBN $90,61961742$.

AN ASTERISK BEFORE THE NUMBER MEANS "TO APPEAR" 
